Patrick Bossuyt

AComparison of Probabilistic

Unfolding Theories

for Paired

Comparisons Data

Springer-Verlag 




\section{A COMPARISON OF \\ PROBABILISTIC UNFOLDING THEORIES \\ FOR PAIRED COMPARISONS DATA}

een wetenschappelijke proeve op het gebied van de Sociale Wetenschappen

\section{Proefschrift}

ter verkrijging van de graad van doctor aan de Katholieke Universiteit te Nijmegen, volgens het besluit van het college van decanen in het openbaar te verdedigen op dinsdag 22 mei 1990, des namiddags te 3.30 uur

door

Patrick Maria Medard Bossuyt

geboren op 10 mei 1959

te Mortsel

NICI

The Nijnegen Instiutuc for Cognition Research and Information Technology, Division of Mathematical Models and Decision Theory 
Promotor: Prof, Dr. E.E.Ch.I. Roskam

This study was supported by the Netherlands Foundation for Scientific Research (NWO, november 1983-may 1987, project no. 40-30). 
Patrick Bossuyt

\section{A Comparison of Probabilistic Unfolding Theories for Paired Comparisons Data}

Springer-Verlag Berlin Heidelberg New York London Paris Tokyo Hong Kong 
Author

Patrick Bossuyt

Center for Clinical Decision Making

Medical Faculty, Erasmus University Rotterdam

P.O. Box 1738, 3000 DR Rotterdam, The Netherlands

ISBN 3-540-52491-6 Springer-Verlag Berlin Heidelberg New York ISBN 0-387-52491-6 Springer-Verlag New York Berlin Heidelberg

This work is subject to copyright. All rights are reserved, whether the whole or part of the material is concerned, specifically the rights of translation, reprinting, re-use of illustrations, recitation, broadcasting, reproduction on mıcrofilms or in other ways, and storage in data banks. Duplicatıon of this publication or parts thereof is only permitted under the provisions of the German Copyright Law of September 9, 1965, in its version of June 24, 1985, and a copyright fee must always be paid. Violations fall under the prosecution act of the German Copyright Law.

O Springer-Verlag Berlın Heıdelberg 1990

Prunted in Germany

Prıntıng and bındıng: Druckhaus Beltz, Hemsbach/Bergstr.

2126/3140-543210 - Printed on acid-free paper 
to Hannah 



\section{Preface}

Some data-analytic methods excel by their sheer elegance. Their basic principles seem to have a particular attraction, based on a intricate combination of simplicity, deliberation, and power. They usually balance on the verge of two disciplines, data-analysis and foundational measurement, or statistics and psychology.

To me, unfolding has always been one of them. The theory and the original methodology were created by Clyde Coombs (1912-1988) to describe and analyze preferential choice data. The fundamental assumptions are truly psychological; Unfolding is based on the notion of a single peaked preference function over a psychological similarity space, or, in an altemative but equivalent expression, on the assumption of implicit comparisons with an ideal alternative.

Unfolding has proved to be a very constructive data-analytic principle, and a source of inspiration for many theories on choice behavior. Yet the number of applications has not lived up to the acclaim the theory has received among mathematical psychologists. One of the reasons is that it requires far more consistency in human choice behavior than can be expected.

Several authors have tried to attenuate these requirements by tuming the deterministic unfolding theory into a probabilistic one. Since Coombs first put forth a probabilistic version of his theory, a number of competing proposals have been presented in the literature over the past thirty years.

This monograph contains a summary and a comparison of unfolding theories for paired comparisons data, and an evaluation strategy designed to assess the validity of these theories in empirical choice tasks.

Chapter 1 contains a classification of the existing probabilistic unfolding theories in which a distinction is made between random configuration theories and random response theories.

Chapter 2 presents an organized description of possible properties of probabilistic choice behavior. Among these are the familiar probabilistic versions of the transitivity property, as well as properties tied to Coombs' definition of the ideal altemative, and properties that apply in unidimensional unfolding representations only. For each probabilistic unfolding theory reintroduced in Chapter 1, and for each property, proofs are presented on whether or not the theory expects that property to hold. 
In Chapter 3, the kernel of the evaluation strategy is presented. To assess the validity of a particular probabilistic unfolding theory, one should look at the goodness-of-fit between models of data and models of critical properties of probabilistic choice behavior.

The strategy itself is discussed in greater detail in Chapter 4. It is based on regarding properties of probabilistic choice behavior as conditional ordinal restraints in estimating choice probabilities, and uses isotonic regression, a branch search strategy and the generalized likelihood ratio principle. The technique is applied to some models of data presented in the literature.

Chapter 5 contains a report on a collection of new paired comparisons tasks in which the evaluation strategy presented in the earlier chapters was used.

After completing my psychology study at the University of Ghent, a grant from the Netherlands Foundation for Scientific Research Nwo (1983-1987, no. 40-30) enabled me to undertake this study at the Mathematical Psychology Group of the University of Nijmegen, presently integrated in the Nijmegen Institute of Cognition Research and Information Technology NICI.

I would like to record my gratitude for Geert De Soete, who has put me on the tracks, for Professor Edward Roskam, for his inspiring supervision, for Math Candel, who has been an arduous beta-tester of PSTRIX and has pointed out several flaws in earlier versions of the package and the manuscript. I am indebted to Frans Gremmen and his colleagues from the Nijmegen GRD for assistance with statistical and programming problems. I want to thank the students that participated in the experiments and Guillaume Vuist for his assistance in running them. Professor Thom Bezembinder, Professor Ivo Molenaar, and Professor Luc Delbeke read the manuscript and helped to improve it.

The Nijmegen Mathematical Psychology Group and the Rotterdam Center for Clinical Decision Making have been stimulating environments to work and live in. To all colleagues, former and present, go my thanks.

Last but certainly not least, I owe a very special debt to all that helped me without even mentioning "probabilistic unfolding", my family and friends in Belgium and the Netherlands.

Rotterdam P.M.B.

February 1990 


\section{Contents}

Preface

vii

$1 \quad$ Approaches to probabilistic unfolding 1

1.1 An overview of probabilistic unfolding theories 3

1.1.1 Random configuration theories 4

1.1.2 Random response theories 8

1.2 Probabilistic unfolding models 9

1.2.1 Models of the Coombs-Zinnes-Griggs theory 10

1.2.2 Random distance models 15

$\begin{array}{ll}\text { 1.2.3 Strong unfolding models } & 17\end{array}$

1.2.4 Midpoint unfolding models 21

$\begin{array}{lll}1.2 .5 \text { Summary } & 22\end{array}$

2 Properties of probabilistic choice behavior 25

2.1 Properties 26

2.1.1 Stochastic transitivity 26

2.1.2 The ideal point 29

2.1.3 Unidimensional unfolding 36

2.2 Proofs 41

2.2.1 Stochastic transitivity 41

2.2.2 The ideal point 46

2.2.3 Unidimensional unfolding 51 
3.1 Testing probabilistic choice theories and models 54

3.1.1 Models of theory 55

3.1.2 Models of data 59

3.1.3 Theory versus data: evaluating goodness of fit 61

3.2 Sampling schemes in paired comparison tasks 65

3.2.1 Data collection designs based on specified subjects 65

3.2.2 Data collection designs based on sampled subjects 67

$\begin{array}{ll}3.2 .3 \text { Conclusion } & 70\end{array}$

$4 \quad$ Evaluating properties of probabilistic choice behavior 73

$\begin{array}{lll}4.1 & \text { Preliminaries } & 74\end{array}$

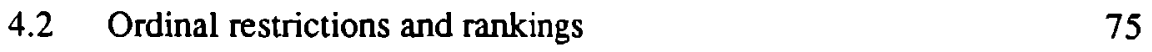

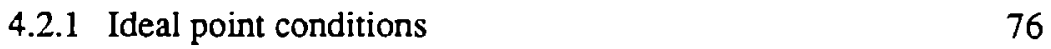

4.2.2 Unidimensional unfolding 76

$\begin{array}{ll}\text { 4.2.3 Stochastic transitivity } & 78\end{array}$

4.3 Maximum likelihood estimates of binomial probabilities under ordinal restrictions $\quad 82$

4.3.1 Basic principles $\quad 82$

$\begin{array}{ll}\text { 4.3.2 The algorithm } & 86\end{array}$

$\begin{array}{ll}\text { 4.3.3 Gebhardt's algorithm } & 93\end{array}$

4.3.4 Application 95

$\begin{array}{lll}4.4 & \text { A branch search strategy } & 98\end{array}$

4.4.1 The branch search principle $\quad 99$

$\begin{array}{ll}4.4 .2 \text { The algorithm } & 100\end{array}$

4.4.3 Implementation 101

4.4.4 Application 103

4.4.5 Extensions 106

4.5 Testing ordinal restrictions on binomial parameters 107

4.5.1 A generalized likelihood ratio test 107

4.5.2 A Monte Carlo approach 110

$\begin{array}{lll}4.6 & \text { Examples } & 113\end{array}$

4.6.1 Stochastic acyclicity 114

4.6.2 Strong stochastic transitivity 116

$\begin{array}{ll}\text { 4.6.3 Characteristic monotonicity } & 118\end{array}$ 
5 An experimental evaluation of probabilistic unfolding theories

5.1 Experiment 1 121

5.1.1 Method

5.1.2 Results

123

5.1.3 Discussion

126

5.2 Experiment 2

128

5.2.1 Method

5.2.2 Results

5.2.3 Discussion

136

5.3 Conclusions

References

Appendix A - PSTRLX

Appendix B - Choice proportions

Appendix C - Distributions

List of Symbols

Author Index 



\section{Approaches to probabilistic unfolding}

The method of paired comparisons is a technique for the collection of data used in a variety of fields such as acoustics, animal ecology, choice behavior, dentistry, economics, epidemiology, food science, marketing, optics, personnel testing, preference testing, psychometrics, sensory testing, sports, taste testing and others (David, 1988). Altematives are presented in pairs to one or more subjects, who are asked to pick one of them. Making such a binary choice is probably the simplest of all choice tasks. For this reason, this method is primarily used in cases where judgements are necessarily subjective. The method is essentially due to Fechner, and several parametric and nonparametric methods for analyzing such data have been proposed ever since. Bradley (1976) and David (1988) have summarized most of these methodological approaches.

Some forty years ago Clyde Coombs $(1950,1964)$ provided the analysis of choice data with a powerful paradigm. Coombs assumed that subjects, when making a choice, are actually comparing the available altematives with an ideal alternative, which is the alternative they actually want, actually need or actually should choose, depending on the choice situation. Asked to choose from two alternatives, neither of which may be the ideal, the subject will choose the alternative least dissimilar from the ideal. Coombs conjectured that subjects differ in the choices they make because they do not necessarily agree on the ideal altemative, but that even subjects who disagree in choice will share the same underlying cognitive pattem of dissimilarities between altematives. Assuming subjective differences in the definition of the ideal, but intersubjectivity in the perception of alternatives, Coombs proposed his "unfolding" theory. The unfolding theory is closely related to the data-representation technique from which it received its name. With this technique, the latent unfolding structure of dissimilarities between ideals and altematives is represented as a set of distances in a psychological space, and a smallest-space representation is aimed at.

In the past decades, the unfolding paradigm has never been absent from the literature on psychological choice, and the amount of methodological contributions based on this paradigm makes it impossible to review them here. The opportunity in Coombs' original unfolding technique of mapping the dissimilarities in a unidimensional space has been complemented with options involving general Euclidean spaces (Bennett \& Hays, 1960; Hays \& Bennett, 
1961) and spaces with ultrametric or path length metrics (De Soete, DeSarbo, Fumas \& Carroll, 1984a, 1984b; Carroll, DeSarbo \& De Soete, 1987). The concept of the ideal point has even inseminated other data analytic procedures, as in Takane, Bozdogan and Shibayama's (1987) proposal for an ideal point approach to discriminant analysis.

Despite the widespread appeal of the unfolding paradigm, Coombs' original unfolding techniques have seen limited application. The principal reason for this absence is to be found in the strong predictions made by the unfolding theory. For example, all choice pattems are expected to be perfectly consistent with a configuration of distances in the underlying psychological space. A consequence of this prediction is that transitivity of choices is never to be violated. If it is, an unfolding representation is not possible. Another difficulty arises when the decision maker of interest in an application is not a single subject, but a population of subjects. If a single set of underlying dissimilarities governs the choices of the members of this population, then these members should not differ in the choices they actually make. This assumption is barely tenable. A related problem exists if a single subject is asked to make repeated choices: The subject's choices need not be consistent over repeated presentations of the same option set. In general, these predictions tumed out to be strong conditions on sets of data, and made a successful use of Coombs' unfolding technique a rare event.

There have been two distinct approaches in tuming Coombs' original unfolding techniques into less demanding data analytical procedures. Both approaches accept the existence of inconsistencies, and view them as marginal deviations from the basic unfolding structure. In one of these approaches, the supposedly small violations of the unfolding theory are left largely unexplained. These procedures simply accept the possibility of inconsistencies occurring, and try to construct an unfolding representation that is as close as possible to the data, in the sense of some criterion to be optimized.

Techniques devised in this approach are in some respect just "black boxes", or scaling procedures. A major example is the non-metric multidimensional scaling (MDS) approach to row conditional data. At an early stage in their development, non-metric techniques for smallest-space representations of dissimilarity data (Shepard, 1962; Kruskal, 1964) were adapted to suit the unfolding paradigm (e.g. Roskam, 1968; Carroll, 1972; Heiser \& De Leeuw, 1981). Similarly, metric MDS approaches have also been used (Schönemann, 1970). Other techniques are based on a coefficient of scalability, which can be probabilistic in nature. An example is the $H$ coefficient in MUDFOLD (Van Schuur, 1984), which is based on a comparison of the observed number of "errors" with the number expected under an independence assumption. MUDFOLD also tries to find a representation for a maximal subset of the alternatives, instead of for the full set, as in "classical" unfolding. 
In the second approach to an unfolding representation of data that are not in accordance with the requirements of the theory, theoretical assumptions are added to the basic unfolding theory, and the representational technique is closely linked to them. These assumptions either attempt to explain the origin of the inconsistencies, or try to predict particular regularities in the probabilities of choosing alternatives. Furthermore, the representational technique is conceived from these assumptions. This second approach, which we will call the probabilistic one, will be the concem of this dissertation. In particular, a comparison of the distinct probabilistic assumptions presented to accommodate the unfolding theory to small, irregular inconsistencies will be aimed at.

The unfolding theory has been used with a wide variety of data, and probabilistic versions are as numerous. Most of these probabilistic versions have been developed for paired comparisons data and representations in Euclidean spaces, though most of these spaces are unidimensional. Since this majority of probabilistic unfolding theories includes both the oldest proposal (Coombs, 1958) and the largest number of competing theories, we will restrict our comparison to the members of this majority.

In the remainder of this chapter we will introduce a series of probabilistic unfolding theories for paired comparisons data - as well as specifications of models of these theories - that have been introduced in the literature during the past three decades. Section 1.1 contains a classification and a reintroduction. Section 1.2 offers a more detailed discussion of the model specifications of these probabilistic unfolding theories for paired comparisons data.

\subsection{PROBABILISTIC UNFOLDING THEORIES}

The subdivision used in this overview is modeled after a distinction made by Croon (1983). Croon's subdivision itself was inspired by a more general classification of probabilistic utility theories by Luce and Suppes (1965). Luce and Suppes distinguished random utility theories from random response theories, based on the way in which a probabilistic choice theory attempts to describe random variability in choices.

In random response theories, choice variability is described by thinking of choices as Bemoulli trials. The probability of success in such a trial is expressed algebraically as a function of the utilities of the choice alternatives involved. In random utility theories, choice variability is explained by means of random utility variables; the utilities of the feasible altematives at the moment of choice are random variables. The unfolding pendants of these random utility theories will be called random configuration theories. They assume that the pattern of dissimilarities between ideals and altematives at the moment a choice has to be made is a random variable. 


\subsubsection{Random configuration theories}

Within the random configuration theories, we will distinguish between random coordinate theories and random distance theories. The former start from randomness assumptions at the level of the coordinates of ideals and alternatives, the latter from comparable assumptions at the level of the distances.

\section{Random coordinate theory}

The first theory of probabilistic unfolding was developed by Coombs (1958) himself. Such an early introduction of a probabilistic version of the unfolding theory can hardly be surprising. Psychologists had always been aware of the difficulties in applying strictly algebraic approaches to the measurement of behavior-related variables. Only on rare occasions data seem to be willing to agree with assumptions made in algebraic approaches. Due to this ubiquity of "error", psychological measurement has witnessed, ever since the days of Fechner, an ever increasing plethora of probabilistic scaling approaches. Among the best known probabilistic approaches to measurement in psychology are Thurstone's collection of techniques (Thurstone, 1927, 1959).

At the foundation of many of Thurstone's techniques lies what he had called the "Law of Comparative Judgment for paired comparisons". Thurstone associated the presentation of an altemative in a paired comparisons task with a so-called "discriminal process". The latter can be thought of as a scale value or coordinate on some underlying psychological continuum, which is subject to random variability. The origin of this variability was left unexplained; it could be any non-systematic momentary influence on the perception of the stimulus. In Thurstone's view, the presentation of two stimuli resulted in two discriminal processes, and with each process a momentary coordinate value could be associated. The stimulus with the highest momentary value would be chosen. An elaboration of Thurstone's ideas can be found in Bock \& Jones (1968).

Coombs provided a minor but crucial adaptation to Thurstone's conceptual framework. Instead of two discriminal processes, one for each stimulus, Coombs foresaw three. In addition to the two discriminal processes for both alternatives, a third discriminal process had to be associated with the ideal alternative of the choosing subject. Instead of choosing the stimulus with the highest momentary coordinate value, the subject would pick the stimulus for which the distance to the ideal, computed on the momentary coordinate values, was smaller.

Coombs conceptual change was crucial because a simple comparison of the magnitudes of the coordinate values on the psychological continuum became irrelevant for the choice process, replaced as it was by a comparison of distances 
to the ideal as the criterion for making a choice. Coombs' approach is based on the idea that a random variability of coordinates can be considered as reflecting the subject's uncertainty conceming the ideal alternative and the alternatives presented.

At first, Coombs did not present a fully developed specification of how a model of this probabilistic unfolding theory would look like, but a few years later a joint effort of Coombs, Greenberg and Zinnes (1961) led to such a characterization. A decade later, a more elaborate model specification of this random coordinate theory was presented by Zinnes and Griggs (1974).

In the Zinnes \& Griggs random coordinate theory, the distributions for the coordinates of the altematives on each dimension are assumed to be of a shift family; they are identical except for an additive factor in the expected values. The coordinate of the ideal is also thought of as a normally distributed random variable, but the variance of its distribution is allowed to differ from the variance of the distributions for the coordinates of the alternatives.

The fact that the coordinates were regarded as normally distributed random variables in all models of Coombs' theory will have surprised no one. Despite the absence of a solid theoretical basis, the normal distribution had also been used by Thurstone, and has never been absent since in probabilistic approaches to scaling and measurement in the behavioral sciences.

In addition to formulating a model for Coombs' original theory, Zinnes \& Griggs suggested one variation of this theory's assumptions. Instead of assuming that only one momentary value of the ideal coordinate is used in computing distances from altematives to the ideal, they offered an alternative assumption. According to Zinnes and Griggs, on some occasions the assumption that two values are used, one for each distance, would be warranted. The first assumption (one value) may be sensible if, for example, the altematives are simultaneously presented. If the alternatives are presented sequentially, the second assumption (two values) may be more reasonable.

In terms of a sampling scheme, this variation implies that one value of the ideal coordinate is sampled for computing the distance to the first altemative, and a second value for computing the distance to the remaining altemative (double sampling), whereas in Coombs' proposal only one value is sampled (single sampling).

Although essentially variations on Coombs' original random coordinate theory, two related theories deserve to be mentioned on their own. One theory is the random ideal coordinate theory. This is a borderline case of Coombs' theory with the assumption of single sampling of the ideal, in which the distributions of the coordinates for altematives are degenerated. This means that, according to this theory, there is no random variability in the location of alternatives in the psychological space; all choice variability has to be attributed to a random variability in the coordinates of the ideal alternative. 
A model specification of this random ideal coordinate theory for a unidimensional space was presented by Bechtel (1968). However, Bechtel did not do so with the intention of defining a variation of Coombs' theory. Bechtel arrived at his model by adding parametric assumptions to a monotonic relation between choice probabilities and interaltemative midpoints, analyzed by Greenberg (1965). Because of the selection of the normal distribution for the ideal coordinate, Bechtel's model is a limiting case of Zinnes \& Griggs' model.

De Soete, Carroll, and DeSarbo (1986) also provided a model specification of the random ideal coordinate theory, allowing of a Euclidean psychological space of higher dimensionality. They called their model the wandering ideal point model. A highly similar model specification was offered by Gaul (1987).

A second variation on Coombs' probabilistic unfolding theory is based on an assumption that is the opposite of the one Bechtel's model is based on. If all choice variability is attributed to a random variability of values of the coordinates for the altematives, a version of Coombs' theory is obtained that is very similar to Thurstone's theory. Here once again choices are govemed by a comparison of the simple magnitudes of the coordinates of the altematives, though not absolutely but relative to the location of the ideal.

\section{Random distance theory}

If coordinates are random variables, any metric defined on altematives through their coordinates will also be a random variable. This means that, in a way, Coombs' theory and its descendants can also be characterized as random distance theories. The reason we prefer to call them random coordinate theories is that their basic premises are formulated in terms of randomness assumptions on the coordinate level. In this view, the members of a second class of theories properly deserve to be called random distance theories. The premises of these random distance theories are made on the level of the ideal-altemative distances, instead of on their respective coordinates.

Though differing from Coombs' random coordinate theory, the random distance theories proposed in the literature are similarly indebted to Thurstone's (1927) Law of Comparative Judgment. Instead of attributing choice variability to a random uncertainty in locating stimuli and ideal in the psychological reference space, these theories assume a random uncertainty in assessing the magnitude of distances between ideal and alternatives.

The first random distance theory was proposed by Ramsay (1980b). Ramsay has invested considerable effort in developing a probabilistic approach to multidimensional scaling of dissimilarities (Ramsay, 1969, 1977, 1978a, 1978b, 1980a, 1980b, 1982). He saw an inevitable relation between the maturing of a data analysis technology, the explication of the error model involved in the fitting process, and some provision for choice among error 
models. In this development, Ramsay has shown himself to be a determinate proponent of the maximum likelihood approach.

Ramsay's first proposal for a maximum likelihood approach to multidimensional scaling (Ramsay, 1969) was based on assumptions similar to the ones made in the Coombs-Zinnes-Griggs approach to probabilistic unfolding. Crediting Thurstone, Ramsay developed a coordinate-oriented strategy, in which coordinates were to be interpreted as values on relevant attributes in the discrimination process. Ramsay assumed independent normal distributions for these values, with identical variances over altematives and dimensions.

In subsequent papers, Ramsay abandoned his original strategy. The reasons for this change are nowhere made explicit in his writings, but some grounds are to be gathered in his plea for a distance-oriented error model. First of all, Ramsay (1977) stated, observed dissimilarities (distances) tend to be nonnegative, either because they have a clearly defined origin or because they are transformable to a nonnegative form. Second, the first moment of an error distribution should be relatively close to the errorless distance. In particular, if one assesses the dissimilarity of two equal stimuli, the first moment of the error distribution should approach zero (The latter is not true in the CoombsZinnes-Griggs approach). Ramsay invokes the subjective Weber Law in a third argument: The standard deviation of the error distribution should be proportional to the errorless distance.

On these grounds, Ramsay proposed the lognormal error distribution, as a first guess at the behavior of dissimilarity data. In a later paper, he examined the feasibility of this approach in scaling pairwise preferences, using the unfolding paradigm (Ramsay, 1980b). At that time a fourth line of argument was appended to the three mentioned earlier: A lognormal error distribution for the distances can be thought of as the distribution of the logarithms of normally distributed disutilities on an interval scale.

It should be noticed that Ramsay collected pairwise preference ratings, not paired comparisons data. However, Croon (1983) has used Ramsay's line of argument for the latter kind of data. Croon also presented a related model of the random distance theory, based on a Weibull error distribution.

A related proposal comes from DeSarbo, De Soete and Eliashberg (1987), though they did not exactly specify distributional assumptions for the idealalternative distances. DeSarbo and his colleagues viewed the perceived disutility of an altemative as the squared distance to an ideal with an independent, normally distributed, additive error term. For this reason we prefer to classify this model as a random distance model. Its distributional assumption implies that perceived dissimilarities may become negative, which, as Ramsay noted, is something of an oddity. 


\subsubsection{Random response theories}

Random coordinate and random distance assumptions are both variations on Thurstone's theme of discriminal processes goveming choices. This distinguishes random configuration theories from a second class of theories, which we will call random response theories. The distinction between random coordinate theories and random response theories parallels a more general partition of the set of probabilistic choice theories into random utility and random response theories, identified by Luce \& Suppes (1965).

Unlike random utility theories, random response theories are not built on assumptions of momentary fluctuations in either coordinates or distances. Instead, these theories assume that choices are inherently random, and to this basic assumption they add others that impose a particular structure on choice probabilities. The magnitude of the probability of choosing one altemative instead of a second is assumed to be functionally related to key elements of the unfolding representation, such as the distances from the ideal to the feasible alternatives, or the distance from the ideal to the interaltemative midpoint. Usually these structural assumptions are borrowed from scaling procedures unrelated to unfolding, a major example being the Fechnerian scaling approach.

\section{(1) Strong unfolding theory}

The main postulate in the Fechnerian scaling approach is that "equidiscriminable pairs of stimuli are represented by equal (scale) differences" (Falmagne, 1971). This idea has been used in the development of a Fechnerian or strong utility theory for probabilistic choice behavior, in which equal choice probabilities are represented by the same difference between utility values associated with the altematives (Davidson \& Marschak, 1959; Block \& Marschak, 1960; Luce \& Suppes, 1965).

In an unfolding context, the Fechnerian approach leads to the condition that equal probabilities of choice are to be represented by the same difference between two ideal-to-alternative distances, transformed to utilities. Because of its basic similarity with the strong utility theory, the resulting probabilistic unfolding theory will be called the strong unfolding theory (Bossuyt \& Roskam, 1986).

Several model specifications of the strong unfolding theory have been presented in the literature (Schönemann \& Wang, 1972; Bechtel, 1976; Jansen, 1981; Cooper \& Nakanishi, 1983; Andrich, 1985). Basically, the principal differences between these strong unfolding models can be traced back to different specifications of the function transforming ideal-alternative distances to utilities (cf. Section 1.2.3). 
The applicability of the Fechnerian approach in describing choice probabilities has been challenged by several authors. Their main argument is that choice probabilities cannot always be adequately described through differences in utility alone; the dissimilarity between altematives also affects the probabilities of choosing from them (e.g. Debreu, 1960). Keeping the utility difference the same, the choice variability is assumed to increase when the alternatives to be chosen from get more dissimilar. These theories of probabilistic choice behavior have been called moderate utility models (Halff, 1976).

Translations of the moderate utility theory to the unfolding context have not been presented thus far, though it would not be difficult to define one. Compared to the strong unfolding theory, such a translation would imply that the interalternative distance is included as a third parameter in the description of choice probabilities.

A third member of the class of random response theories is very similar to the moderate unfolding theory. In the midpoint unfolding theory, equal choice probabilities are described by the same difference between the ideal coordinate value and the value of the midpoint between altematives. For a particular pair of alternatives, choice probabilities are expected to become more extreme (i.e. closer to 1 or 0 ) when the distance from the ideal to the midpoint increases. Specifications of models of this midpoint unfolding theory have been presented by Bechtel (1968), Sixtl (1973) and Jansen (1981). The latter two are both originally inspired by the Rasch item-response theory.

\subsection{PROBABILISTIC UNFOLDING MODELS}

The following two subsections offer a more detailed analysis of model specifications of random coordinate and random distance theories: the Zinnes-Griggs model and the Ramsay model respectively. A third subsection presents more details on strong unfolding models. More specifically, the question of selecting a proper preference function is discussed. A fourth section briefly discusses the relation between the midpoint unfolding theory and the moderate unfolding theory.

Before we continue, we will introduce some notational conventions. Sets will be notated by single Latin letters in uppercase (e.g. $X$ ), elements (or points) by single letters in lowercase (e.g. $x, y$ ). If possible without creating confusion, we will use the same lowercase letters to denote values of functions with the set of these elements as their domain (e.g. $x, y$ for the coordinate values of the points $x, y$ ). In distinguishing between elements or sets we will occasionally use subscripts (e.g. $x_{1}, x_{2}$ ). The ideal altemative will be denoted by the last letter 
of the alphabet, $z$, subscripted if necessary.

We will use shorthand notation for the metric - $d_{x y}$ for the distance $d(x, y)$ between $x$ and $y$ - and for the choice probabilities, $p_{x y}$ for the probability of choosing $x$ out of $\{x, y\}$. Vectors will be described using bold typefaces (e.g. $x)$. In describing random variables we will use uppercase Latin letters, such as $D_{x y}$ if the distance is a random variable, or $\mathbf{X}$ for a random coordinate vector. The same letters in lowercase will be used for the errorless values, say $d_{x y}, \mathbf{x}$.

Throughout a choice between an alternative $x$ and an altemative $y$ in a paired comparisons task will be the subject of interest, under the condition that only one alternative can be chosen, the no-choice option being eliminated. So we have $p_{x y}+p_{y x}=1$. For subject indices we will use the letters $i, j$, or $k ; p_{x y}$ stands for the probability that subject $i$ chooses the altemative $x$ after a particular presentation of the pair $\{x, y\}$.

The structure $\langle X, p\rangle$, consisting of a finite set $X$ and a probability function $p$ defined on $X \times X$, will be called a binary choice probability (BCP) structure. The structure $\langle X, P\rangle$, consisting of a finite set $X$ and a set $P$ of probability functions $\left\{p_{i}\right\}$, all defined on $X \times X$, will be called a binary choice probability system.

The relation between probabilistic unfolding theories, models of these theories, and models of data will be discussed in Chapter 3 later on. At this point it will suffice to keep in mind that a model of a theory will be thought of as a possible realization of this theory. This is a settheoretical structure of the appropriate type in which all the assumptions of the theory are satisfied. In probabilistic unfolding, such a settheoretical structure - a model - will at least contain a BCP system, and a metric defined on the union of the set of altematives and ideals.

A model of one probabilistic unfolding theory will be called equivalent to a model of a second theory if both models are able to represent the same BCP system with the same metric.

\subsubsection{Models of the Coombs-Zinnes-Griggs theory}

In this section we will explore models in the tradition of Coombs' original proposal for a probabilistic unfolding theory (Coombs, 1958). The model for a general, $r$-dimensional Euclidean space will be discussed first. Next some special cases will be explored.

The general model

In its most general formulation, the Coombs-Zinnes-Griggs approach assumes that the coordinates of the ideal alternative $z$ and altematives $x$ and $y$ are random variables, $Z, X$, and $Y$ respectively. The Zinnes-Griggs model, as 
discussed in the 1974 paper, is considerably less general. All coordinate vectors are assumed to follow independent multivariate normal distributions with scalar variance-covariance matrices. Furthermore, all variance-covariance matrices for the coordinate vectors of the altematives are assumed to be equal. The matrix for the distribution of the ideal's coordinate vector is allowed to differ. Without loss of generality, we will assume that on each dimension $2\left(\sigma_{z}^{2}+\sigma_{x}^{2}\right)=1$.

We will reintroduce the Coombs-Zinnes-Griggs model, as proposed by Zinnes and Griggs (1974). The case of a r-dimensional Euclidean space will be elaborated first, followed by a discussion of representations in a unidimensional psychological space. Obviously, the second situation is but one case of the first, but there are some differences in Zinnes and Griggs' approaches to each situation. Later on, models of related approaches will be shown to be limiting cases of the Coombs-Zinnes-Griggs model.

When asked to choose between $x$ and $y$, the subject is assumed to sample coordinate vectors for both altematives, and either one or two coordinate vectors for the ideal. These vectors are then used to compute Euclidean distances as

$$
D_{u}^{2}=\left(Z_{1}-X\right)^{\prime}\left(Z_{1}-X\right) \quad D_{r y}^{2}=\left(Z_{2}-Y\right)^{\prime}\left(Z_{2}-Y\right),
$$

in which $Z_{1}$ stands for the coordinate vector of the ideal used with the first alternative, and $Z_{2}$ for the coordinate vector of the ideal used with the second. Obviously, these squared distances between the ideal $z$ and altematives $x$ and $y$ respectively are also random variables. Because of the assumptions made earlier, it can be shown that they have noncentral chi-square distributions, with the dimensionality $r$ of the Euclidean space as degrees of freedom, and the doubled, squared errorless distances $2 d_{z}^{2}, 2 d_{x y}^{2}$ as noncentrality parameters.

In the unidimensional case, the distances can be expressed as the absolute values of the corresponding coordinate differences,

$$
\begin{aligned}
D_{\mathbf{x}} & =\left|Z_{1}-X\right| & D_{x y} & =\left|Z_{2}-Y\right| \\
& =\left|T_{\mathrm{a}}\right| & & =\left|T_{\mathrm{z} y}\right| .
\end{aligned}
$$

As a consequence of Coombs-Zinnes-Griggs' assumptions of single ideal point sampling referred to earlier, the cumulative distribution function for $\left(T_{2 x}, T_{x y}\right)$ is a bivariate normal one, with variance-covariance matrix

$$
\frac{1}{2}\left(\begin{array}{cc}
1 & \rho_{\tau_{u} T_{\eta}} \\
\rho_{T_{u} T_{\eta}} & 1
\end{array}\right) .
$$


In a Coombs-Zinnes-Griggs model, the probability $p_{x y}$ of choosing $x$ instead of $y$ can be expressed as

$$
p_{x y}=\operatorname{Pr}\left[D_{x x} \leq D_{x y}\right]
$$

In the unidimensional case, the choice probability can alternatively be expressed as

$$
p_{x y}=\operatorname{Pr}\left[\left|T_{z x}\right| \leq\left|T_{z y}\right|\right] .
$$

Zinnes and Griggs have provided an interesting result for this unidimensional case, by proving that [1.5] can be expressed as a function of the errorless coordinate values and the correlation between the coordinate differences:

$$
\begin{aligned}
p_{x y} & =G\left(z, x, y, \rho_{T_{z} T_{z}}\right) \\
& =\Phi\left(\alpha_{z x y}\right) \cdot \Phi\left(\beta_{x y}\right)+\Phi\left(-\alpha_{z y}\right) \cdot \Phi\left(-\beta_{x y}\right),
\end{aligned}
$$

in which $\Phi($.$) stands for the standard normal cumulative distribution function,$ with

$$
\begin{aligned}
& \alpha_{z y}=\frac{2 z-x-y}{\sqrt{1+\rho_{T_{u x} T_{n y}}}} \\
& \beta_{x y}=\frac{x-y}{\sqrt{1-\rho_{T_{u} T_{\eta y}}}} .
\end{aligned}
$$

\section{Case I: double sampling of the ideal coordinate}

The expression for the choice probability [1.4] can be reformulated as

$$
P_{z y}=\operatorname{Pr}\left[\frac{D_{u}^{2}}{D_{y y}^{2}} \leq 1\right] .
$$

In the case of double sampling of the ideal coordinate vector, the joint distribution of $\left(D_{z}^{2}, D_{x}^{2}\right)$ has a zero correlation parameter. Values for the probability in [1.9] can be calculated using the result that the ratio of two random variables with independent noncentral chi-square distributions has a noncentral $F$ distribution (Patnaik, 1949). The distribution of the ratio in [1.9] has three 
parameters: the noncentrality parameters $2 d_{u}^{2}, 2 d_{y}^{2}$ of the two noncentral chi-square distributions, and the $r$ degrees of freedom.

Zinnes and Griggs acknowledged a previous use of the doubly noncentral $F$ distribution in a related context by Hefner (1958). Ramsay (1969) has explored the properties of this distribution in a multidimensional scaling context, but he did not publish any results on applications. Apparently the doubly noncentral $F$ distribution was much too complex to be of any practical use. Bulgren(1971) has given an expression involving a doubly infinite series of terms, each of which contains the Pearson Incomplete Beta function. To obtain an acceptable level of accuracy, he found it necessary to use between 36 and 903 terms.

Zinnes and Griggs (1974) developed a rather simple expression for equation [1.9], based on an approximation of the noncentral chi-square distribution with a normal distribution. They also presented results that showed that this approximation was reasonably accurate. The approximation improves as the number of degrees of freedom increases, but even with $r=2$, discrepancies are well within $\mathrm{a} \pm .01$ range from the values calculated by Bulgren (1971).

In the unidimensional case, equation [1.6] obviously still holds with the correlation parameter reduced to zero. The choice probability can altematively be expressed as:

$$
p_{x y}=\Phi\left(d_{z y}+d_{z x}\right) \Phi\left(d_{x y}-d_{z z}\right)+\Phi\left(-d_{x y}-d_{z z}\right) \Phi\left(d_{x z}-d_{x y}\right)
$$

Case II: single sampling of the ideal coordinate

A model of the random coordinate theory with the assumption of single sampling of the ideal coordinate vector has not been explored for the general multidimensional case. The squared distances still have noncentral chi-square distributions but since these distributions are correlated, expression [1.9] for the choice probabilities becomes much more cumbersome to evaluate.

Zinnes-Griggs have studied the unidimensional case. In this case, the joint distribution of $\left(T_{z}, T_{n}\right)$ has a nonzero correlation parameter, which, due to the assumptions made for the general model, is equal to

$$
\rho_{T_{\mathbf{z}} T_{m}}=\frac{\sigma_{z}^{2}}{\sigma_{z}^{2}+\sigma_{x}^{2}}
$$

According to Zinnes \& Griggs, the variance of the distribution for the coordinate of the ideal can be either greater than, equal to, or strictly smaller than the variance of the distributions for alternatives, depending on whether or not the subject has a clear conception of what the ideal altemative is, or should be. The following relationships can be deduced from [1.11]: 


$$
\begin{aligned}
& \sigma_{z}=\sigma_{X} \Rightarrow \rho_{T_{u} T_{r y}}=\frac{1}{2} \\
& \sigma_{z}>\sigma_{X} \Rightarrow \rho_{T_{u} T_{r y}}>\frac{1}{2} \\
& \sigma_{z}<\sigma_{X} \Rightarrow \rho_{T_{u} T_{\eta p}}<\frac{1}{2} .
\end{aligned}
$$

Using equations [1.6-1.8], binary choice probabilities can easily be evaluated.

$$
\text { Case III: ideal coordinate only, single sampling }
$$

A special case of the Coombs-Zinnes-Griggs approach can be obtained by assuming degenerate distributions for the altemative coordinate vectors. The multidimensional version of this case has been studied by De Soete, Carroll and DeSarbo (1986), who baptized their model the "wandering ideal point model". They obtained a fairly simple expression for the choice probability:

$$
p_{x y}=\Phi\left(\gamma-\frac{1}{2}\left(\mathbf{y}^{\prime} \mathbf{y}-\mathbf{x}^{\prime} \mathbf{x}\right)-2(\mathbf{y}-\mathbf{x})^{\prime} \mathbf{z}\right)
$$

with

$$
\gamma=4(\mathbf{y}-\mathbf{x})^{\prime} \Sigma(\mathbf{y}-\mathbf{x})
$$

where $\Sigma$ stands for the covariance matrix of the coordinate distributions. The specification of a "probabilistic ideal point model" by Gaul (1987) is highly similar.

Zinnes and Griggs did not explicitly discuss a model for the unidimensional version of this case, but it follows easily from the relation in [1.5]. Let $m_{\mathrm{xy}}$ stand for the midpoint between alternatives $x$ and $y$, with coordinate value $(x+y) / 2$, defined through the equality

$$
d_{x m_{x y}}=d_{y m_{x y}} .
$$

Define a function $\theta(x)$ over the reals, with

$$
\begin{array}{ll}
\theta(x)=-1 & \text { for } x<0 \\
\theta(x)=0 & \text { for } x=0 \\
\theta(x)=1 & \text { for } x>0 .
\end{array}
$$


Expression [1.5] can be rewritten as

$$
\begin{aligned}
P_{x y}= & \operatorname{Pr}\left[\left|T_{z \mid}\right| \leq\left|T_{x y}\right|\right] \\
= & \operatorname{Pr}\left[2\left(Z-m_{x y}\right) \theta(y-x) \leq 0\right] \\
= & \operatorname{Pr}\left[\sqrt{2}\left(\left(Z-m_{x y}\right) \theta(y-x)-\left(z-m_{x y}\right) \theta(y-x)\right) \leq\right. \\
& \left.\quad-\sqrt{2}\left(z-m_{x y}\right) \theta(y-x)\right] \\
= & \Phi\left(\sqrt{2}\left(z-m_{x y}\right) \theta(x-y)\right) \\
= & \Phi\left(\sqrt{2} d_{1 m_{x y}} \theta\left(d_{x y}-d_{z x}\right)\right) .
\end{aligned}
$$

Basically the same model for this limiting case of the Coombs-Zinnes-Griggs theory was presented by Bechtel (1968). He arrived at it by adding parametric assumptions to a relation between choice probabilities and interaltemative midpoints, detected by Greenberg (1965).

$$
\text { Case IV: coordinates of the alternatives only, single sampling }
$$

If we, within the Coombs-Zinnes-Griggs approach, are willing to assume that the distribution of the ideal coordinate vectors degenerates, once more the distributions of the coordinate differences and, hence, the distances will be independent. Any model of this theory will be equivalent to a model of Case I (double sampling of the ideal). This means that if an appropriate set of distributions exist for a BCP system to make it a model of the theory Case IV, a model of the Case I theory will also exist.

\subsubsection{Random distance models}

In a random distance theory, distances from the ideal to altematives are assumed to be random variables. So a random distance model is a BCP system for which there exists distributions for $D_{z x}, D_{r y}$, such that relation [1.4] holds for the binary choice probabilities.

The approach followed by Ramsay (1977) imposes restrictions on the distributions of the ideal-alternative distances: These are assumed to be independent distributions of a specific parametric family. Croon (1983) has developed a model for choice probabilities in a paired comparisons task, based on Ramsay's strategy. In Croon's model specification, the logarithms of the dissimilarities between alternatives and ideal $\ln \left(D_{z y}\right), \ln \left(D_{z y}\right)$ have normal 
distributions, with variances equal to $1 /\left(2 c^{2}\right)$ ( $c$ a positive real), and expected values $\ln \left(d_{\mathrm{x}}\right), \ln \left(d_{\mathrm{ry}}\right)$ respectively. The expression for the choice probabilities [1.4] can then be formulated as

$$
\begin{aligned}
& p_{x y}=\operatorname{Pr}\left[\ln D_{x} \leq \ln D_{n}\right] \\
& =\operatorname{Pr}\left[\ln D_{x x}-\ln D_{x y} \leq 0\right] \\
& =\operatorname{Pr}\left[\left(\ln d_{x y}-\ln D_{x y}\right)-\left(\ln d_{x z}-\ln D_{x}\right) \leq\left(\ln d_{x y}-\ln d_{z x}\right)\right] \\
& =\Phi\left(c\left(\ln d_{m}-\ln d_{z}\right)\right) \text {, }
\end{aligned}
$$

where $\Phi($.$) stands once more for the standard normal cumulative distribution$ function.

Croon also described another model specification, which is similar to this lognormal one. It satisfies all of Ramsay's assumptions, but uses the Weibull error distribution instead of the lognormal distribution. In this model, the distances $D_{x x}, D_{x y}$ are independent Weibull variates with a common shape parameter $c$ (a nonnegative real) and location parameters $d_{x z}, d_{x y}$ respectively. The latter assumption implies that the deviations $c\left(\ln d_{x x}-\ln D_{x x}\right)$ have standard (Type I) extreme value distributions. Since the distribution of the difference between two standard extreme value distributions is a logistic one (Gumbel, 1961), the choice probability can be expressed as

$$
\begin{aligned}
p_{x y} & =\operatorname{Pr}\left[\ln D_{x y} \leq \ln D_{y y}\right] \\
& =\operatorname{Pr}\left[\ln D_{x y}-\ln D_{y y} \leq 0\right] \\
& =\operatorname{Pr}\left[\left(\ln d_{x y}-\ln D_{y y}\right)-\left(\ln d_{x x}-\ln D_{x y}\right) \leq\left(\ln d_{x y}-\ln d_{z x}\right)\right] \\
& =\frac{d_{z y}^{c}}{d_{x x}^{c}+d_{x y}^{c}} .
\end{aligned}
$$

The expression for the choice probabilities in both the lognormal and Weibull random distance model is a simple function of the two errorless distances from the altematives to the ideal. This makes these models very similar in behavior to the strong unfolding models, to be discussed in the next subsection.

Random distance models with lognormal or Weibull distance distributions cannot be reconciled with assumptions of randomness on the coordinate level. If one starts from the latter, the distribution of the ideal-altemative distance will have a nonzero variance, even if the (errorless) coordinates of the ideal and the altemative coincide. In the Ramsay-Croon model approach, this variance is assumed to be proportional to the errorless distance. As a consequence, 
such a distribution degenerates if the distance from the ideal to the altemative becomes zero.

In their design of a random distance model, DeSarbo, De Soete and Eliashberg (1986) started from the following relation:

$$
\begin{aligned}
\boldsymbol{p}_{x y} & =\operatorname{Pr}\left[D_{x y} \leq D_{z y}\right] \\
& =\operatorname{Pr}\left[d_{z x}+\varepsilon_{z x} \leq d_{x y}+\varepsilon_{y y}\right],
\end{aligned}
$$

with $\varepsilon_{z x}, \varepsilon_{z y}$ two error terms. They assumed that these error terms have normal distributions, with zero location parameters and a standard deviation $\sigma_{z}$, which may differ between subjects. DeSarbo and his colleagues assumed zero correlations between all distributions. With these assumptions they arrived at the following relation

$$
\begin{aligned}
P_{x y} & =\operatorname{Pr}\left[d_{x x}+\varepsilon_{x x} \leq d_{x y}+\varepsilon_{x y}\right] \\
& =\operatorname{Pr}\left[\varepsilon_{x x}-\varepsilon_{z y}<d_{x y}-d_{x x}\right] \\
& =\Phi\left(\frac{2 z^{\prime}(x-y)+x^{\prime} x-y^{\prime} y}{\sigma_{z} \sqrt{2}}\right) .
\end{aligned}
$$

\subsubsection{Strong unfolding models}

In Section 1.1.2 the strong utility theory (Block \& Marschak, 1960; Luce \& Suppes, 1965) was discussed. A strong utility model $\{X, p, u, H\}$ is a BCP structure $\langle X, p\rangle$ for which there exists a real-valued function $u$ over $X$ and a cumulative distribution function $H$ such that for every two elements $x, y \in X$ :

$$
p_{x y}=H(u(x)-u(y))
$$

\section{The strong unfolding model}

If we have a BCP system $\langle X, P\rangle$, and a strong utility model exists with the same distribution function $H$ for each BCP structure $\left\langle X, p_{i}\right\rangle$ in this system, we can arrive at a strong unfolding model if the Fechnerian scale values can be related to ideal-altemative distances in a space $(X, d)$. For this purpose we require the following function, which we will call the preference function:

$$
u_{z}(x)=f\left(d_{2 x}\right)
$$


A preference function (PF) is a real valued function $f$, decreasing over the nonnegative reals, which transforms ideal-alternative distances $d_{\mathrm{zz}}$ in the unfolding model to scale values $u_{r}(x)$ in a Fechnerian strong utility model for binary choice probabilities. With this preference function we can link together the Fechnerian scaling approach and probabilistic unfolding. A strong unfolding model is a BCP system $\langle X, P\rangle$ for which there exists a set of ideal points $Z=\{z\}$, a metric $d$ defined over $X \cup Z \times X \cup Z$, a preference function $f$ and a distribution function $H$ such that

$$
p_{u x y}=H\left(f\left(d_{z_{i} r}\right)-f\left(d_{z, y}\right)\right)
$$

holds for every two elements $x, y \in X$.

Specifications of this strong unfolding model were proposed by Bechtel (1976), Schönemann \& Wang (1972), Jansen (1981), Cooper \& Nakanishi (1983), and Andrich (1985). These specifications differ in the nature of the cumulative distribution function proposed - which is usually either the standard normal or the logistic one - but even more so in their choice of a preference function.

\section{Preference functions}

Coombs' original unfolding technique required complete preference orderings from the subjects and resulted in ordered metric information on the psychological space if the latter was a real line. In so called 'metric' unfolding however, quantitative preference assessments of the altematives are represented in a more direct way as distances between the altematives and an ideal altemative. Consequently a major theoretical problem is the selection of the proper function relating preference evaluations and the corresponding ideal-altemative distances. A related difficulty emerges when an unfolding representation of probabilistic choice is called for. Here also a plausible quantitative relation has to be justified, in the form of a function relating the probability of choosing an alternative to relevant parameters in an unfolding representation.

Basically the same problem presents itself in the metric multidimensional scaling of similarity judgments. In this area the properties of a function relating quantitative dissimilarity assessments, or choice probabilities, and distances representing them has been investigated by such authors as Shepard (1958), Luce (1961) and Krantz (1967). They have developed a theoretical analysis of the cognitive processes that are involved in the judgmental task of assessing dissimilarities. From this analysis they derived what Krantz (1967) has called 'a rational distance function'. 
Rather surprisingly, a similar examination is lacking in the unfolding literature, and the impression prevails that it seems possible to by-pass the question. Most authors seem to have submitted themselves to the view that unfolding is just a collection of related techniques for data reduction and representation. In our view however, the points made by Krantz (1967) and Beals, Krantz \& Tversky (1968) on the scaling of similarity judgments apply just as well to unfolding representations of preferential choice data. The absence of a theoretical justification for the relation between choice probabilities or numerical preference evaluations, and distances makes it hard to evaluate the quality and the interpretability of the resulting representation, if not the validity of the representational technique itself.

In his Theory of Data Coombs (1964) introduced the notion of a single-peaked preference function. This is a function that is defined over the psychological space, reaching a unique maximum at the ideal alternative. Later on, Coombs and Avrunin (1977) have provided necessary and sufficient conditions on a set of alternatives to imply single-peaked preference. They showed that a singlepeaked preference function is a fairly common phenomenon, based on basic psychological principles for the perception and processing of good and bad attributes.

Coombs (1964) showed that his ordered metric unfolding technique required these preference functions to be strictly monotonically descending from the ideal point, symmetrically in all directions. Monotonicity and symmetry are sufficient properties for the unfolding technique to be applicable, and no further characteristics of the single-peaked preference function affect the ordered metric information obtained.

If the function decreases symmetrically in all directions from the ideal point, the single-peaked preference function can be replaced by an equivalent preference function, monotonically descending over the distances to the ideal. If the aim of an unfolding analysis is a metric defined over all the altematives and ideals, and if more than ordinal information is to be extracted from the data, the form of the preference function is critical for the properties of the model constructed.

A first candidate for a preference function in a strong unfolding model is the simple sign transformation:

$$
u_{1}(x)=-d_{x x}
$$

This transformation of distances to Fechnerian scale values had been proposed by Luce (1961) and Krantz (1967), within the context of choice probabilities of similarity judgments. Jansen (1981) has used it in a strong unfolding model.

Schönemann \& Wang (1972) proposed a squared sign transformation in their specification of a strong unfolding model: 


$$
u_{z}(x)=-\left(d_{x x}^{2}\right)
$$

These authors make the following comparison.

" This means, psychologically, that the preference between two stimuli (...) should become more pronounced as their joint distance from the ideal increases. Geometrically it means a shrinkage of peripheral stimulus distances - a distortion somewhat akin to that of an orthographic projection (a plane projection in cartography with eyepoint at infinity). "

(Schönemann \& Wang, 1972)

Cooper \& Nakanishi (1983) and Andrich (1985) adopted the same function. Actually, this quadratic transformation is a popular one in metric multidimensional scaling (see, for example, Carroll, 1972, 1980; Davison, 1977; Srinivasan \& Shocker, 1973). A conceptual justification for using this function is largely absent, but its popularity can likely be attributed to the mathematical convenience of working with squared Euclidean distances.

A more general class of preference functions was proposed by Bechtel (1976):

$$
u_{z}(x)=-\left(d_{z}^{\tau}\right)
$$

Bechtel suggests the successive evaluation of goodness-of-fit indices for unfolding representations obtained under different values for $\tau$. He points to a resemblance with the psychophysical power relation between subjective magnitude judgments and physical measures. However, ideal-altemative distances in an unfolding space do not have an immediate perceptual correlate, and since the psychophysical scaling problem is quite unrelated to unfolding, this resemblance cannot have a substantive interpretation.

An alternative not explored so far within the context of the strong unfolding theory is the logarithm of the inverted ideal-alternative distance,

$$
u_{z}(x)=-\ln \left(d_{z x}\right)
$$

For models of the random distance theories as discussed in Section 1.2.2, there always exists an equivalent strong unfolding model with a logarithmic preference function.

Few specifications of the preference function are inspired by an analysis of the unfolding model itself. Later on, in Chapter 2, we will examine differences 
between strong unfolding models with different specifications of the preference function, in terms of diverging predictions on properties of probabilistic choice behavior.

\subsubsection{Midpoint unfolding models}

The moderate utility theory (Halff, 1976) was discussed in Section 1.1.2. Although no unfolding pendants have been proposed, it is not difficult to do so. A moderate unfolding model would then be a BCP system $\langle X, P\rangle$ for which there exist a set of ideal points $Z=\left\{z_{3}\right\}$, a metric $d$ defined over $X \cup Z \times X \cup Z$, a real valued function $f$ decreasing over the nonnegative reals, an increasing concave transformation $g$ of the nonnegative reals, and a cumulative distribution function $H$ such that

$$
p_{x y}=H\left(\frac{f\left(d_{x z}\right)-f\left(d_{x y}\right)}{g\left(d_{x y}\right)}\right),
$$

holds for every two elements $x, y \in X$.

In the same Section 1.1.2, we discussed the midpoint unfolding theory. A midpoint unfolding model is a BCP system $\langle X, P\rangle$ for which there exist a set of ideal points $Z=\left\{z_{1}\right\}$, a unidimensional metric $d$ defined over $X \cup Z \times X \cup Z$ and a distribution function $H$ such that

$$
p_{x y}=H\left(d_{z m_{g y}} \theta\left(d_{x y}-d_{z z}\right)\right)
$$

holds for every two elements $x, y \in X$, where $m_{\mathbf{r}}$ and $\theta(x)$ are defined as in equation [1.16].

It can be shown that a midpoint unfolding model is always equivalent with a moderate unfolding model with the same cumulative distribution function $H$, a preference function $f(x)=-x^{2}$ and a dissimilarity function $g(x)=2 x$. To prove this, we will just have to show that for a given binary choice probability, the arguments of the cumulative distribution function are the same.

Two cases have to be distinguished:

(a) $d_{x y}=d_{x y}+d_{x x}$, the ideal is located between $x$ and $y$ (a bilateral pair), and

(b) $d_{x y}=\left(d_{x y}-d_{z z}\right) \theta\left(d_{x y}-d_{z}\right)$, the ideal is on either side of $x$ and $y$ (a unilateral pair).

This follows from the unidimensionality assumption. In case (a) 


$$
\begin{aligned}
d_{z m_{x y}} \theta\left(d_{x y}-d_{z z}\right) & =\frac{\left(d_{x y}-d_{z x}\right)}{2} \\
& =\frac{\left(d_{x y}-d_{z x}\right)\left(d_{x y}+d_{z z}\right)}{2\left(d_{x y}+d_{z y}\right)} \\
& =\frac{d_{z y}^{2}-d_{z x}^{2}}{2 d_{x y}}
\end{aligned}
$$

and in case (b)

$$
\begin{aligned}
d_{x m_{z y}} \theta\left(d_{x y}-d_{z x}\right) & =\frac{\left(d_{x y}+d_{z x}\right)}{2} \theta\left(d_{x y}-d_{z x}\right) \\
& =\frac{\left(d_{z y}+d_{z x}\right)\left(d_{x y}-d_{z x}\right)}{2\left(d_{r y}-d_{x z}\right)} \theta\left(d_{r y}-d_{z x}\right) \\
& =\frac{d_{z y}^{2}-d_{z x}^{2}}{2 d_{x y}}
\end{aligned}
$$

which delivers the proof.

\subsubsection{Summary}

A wide variety of probabilistic unfolding theories for paired comparisons data have been presented in the literature ever since Coombs' (1958) original proposal appeared. In this chapter, we have reintroduced most of them within a classification that mirrors an existing distinction between random utility theories and random response theories (Luce \& Suppes, 1965).

We distinguished between two major classes of theories (cf. Table 1.1). One class we called random configuration theories. These theories are based on assumptions of randomness for central elements of the unfolding space. These theories can be subdivided further into random coordinate and random distance theories. The former start from assumptions on the level of the coordinates, the second from assumptions on the level of the distances or perceived dissimilarities from the ideal. Within the random coordinate theories we are offered a choice between the assumptions that the coordinates of either the ideal, the altematives, or both, are random variables. If one assumes that the ideal coordinate is a random variable, there is an additional choice between the assumptions of single sampling and double sampling. 


\section{Table 1.1}

A classification of probabilistic unfolding theories

\section{THEORIES}

MODEL SPECIFICATIONS

RANDOM CONFIGURATION THEOAIES

Random coordinate theory

Case 1 (double sampling ideal)

Case II (single sampling ideal)

Zinnes \& Griggs (1974)

Case III (single sampling ideal only)

Zinnes \& Griggs (1974)

Zinnes \& Griggs (1974),

Bechtel (1968),

De Soete, Carroll \&

DeSarbo (1986),

Gaul (1987)

Case IV (alternatives only)

Zinnes \& Griggs (1974)

Random distance theory
Ramsay (1980b),
Croon (1983),
DeSarbo, De Soete \&
Eliashberg (1987)

RANDOM RESPONSE THEORIES

Strong unfolding theory

Schönemann \& Wang (1972),

Bechtel (1976),

Jansen (1981),

Cooper \& Nakanishi (1983)

Andrich (1985)

Midpoint unfolding theory

Bechtel (1968),

Sixtl (1973),

Jansen (1981)

Any random coordinate theory can altematively be considered to be a random distance theory, since assumptions of randomness on the level of coordinates make a coordinate-based metric a random variable. Yet there are other random distance theories whose assumptions cannot be reconciled with randomness assumptions on the coordinate level. In genereal, random distance unfolding theories are but a subclass of the multitudinous family of random utility theories. 
The second class of probabilistic unfolding theories contains random response theories (Luce \& Suppes, 1965). Unlike random configuration theories, these theories do not start from randomness assumptions for either distances or coordinates. Random response theories just see choices as fit for a probabilistic description, and assume that the choice probabilities in this description can be functionally related to key elements of an unfolding representation. These functional relations are usually borrowed from other probabilistic scaling techniques. The two main theories proposed so far in this category are the strong unfolding theory and the midpoint unfolding theory.

Any random coordinate model is equivalent to a random distance model, and any random distance model is also equivalent to a random response model. If there exists a model of a random coordinate theory for one specific BCP structure, then there will also exist a model of a random distance theory for this BCP structure. Similarly, if there exists a model of a random distance theory for this BCP structure, then there will also exist a model of a random response theory for this structure. 


\section{probabilistic choice behavior}

In Chapter 1 we offered a reintroduction of all proposals for a probabilistic unfolding theory for paired comparisons data that have appeared in the literature during the past thirty years. The classification we used in that review was based on the conceptual equivalencies between theories. We distinguished between random configuration theories, random distance theories, strong unfolding theories, and the midpoint unfolding theory.

Pointing out similarities in concepts and structure between theories, and between model specifications, is but one way of making these approaches comparable. Maybe such a distinction is not the most relevant for a functional comparison, if one wants to determine peculiarities in (probabilistic) choice behavior some of these theories try to account for. A classification based on structure and concepts does not lend itself too easily to a pertinent assessment of a theory's potential for validity. For example, all assumptions of random configuration theories are expressed in terms of entities that are not observable, be they either coordinate or distance based dissimilarities. On the other hand, the assumptions of the random response theories are not related to any aspect of the choice behavior at all, except its probabilistic nature.

This section offers a second classification, or crossclassification, of probabilistic choice theories. This classification is based on properties of choice behavior that are necessarily implied by theories on probabilistic unfolding, or whose violation is implicit in these theories. Due to the probabilistic nature of the theories, all properties are expressed in terms of probabilistic choice behavior, dealing with probabilities of choosing altematives in particular choice situations. Stated formally, these are properties of BCP structures, or BCP systems, and a property is implied by a theory if it is always fulfilled in a BCP system (structure) for which a model of this theory exists. From an alternative point of view, these properties act as necessary conditions for the existence of a model of a probabilistic unfolding theory. This leads us to calling these properties, somewhat loosely, "necessarily fulfilled" in the model, or "required" by a theory. 
The value of these properties both for analysts and theoreticians in the study of choice behavior can be inferred from the way new probabilistic choice theories are introduced in the literature. Several if not most expositions of new probabilistic unfolding theories include a discussion on the theory's implications in terms of assumed properties of probabilistic choice behavior (see, for example, Bechtel, 1968; De Soete, Carroll \& DeSarbo, 1986, DeSarbo, De Soete \& Jedidi, 1987, p.103). It seems that these properties are regarded as valuable indications on what kind of probabilistic choice behavior a probabilistic choice theory wants to describe. For this reason, we will compare the requirements of alternative probabilistic choice theories regarding a series of well-known, and some newly described properties.

We do not want to claim that this approach to comparing theories is new in the literature on probabilistic choice behavior. Marschak (1960) and his colleagues, for example, have explored several of the properties that will be used in this section, with an intention similar to ours, and in their 1965 review of probabilistic choice theories, Luce and Suppes used a related approach. The properties will be discussed in Section 2.1. All proofs will be given at the end of this chapter, in Section 2.2.

\subsection{PROPERTIES}

We will distinguish between three classes of properties. The first class (Section 2.1.1) contains probabilistic formulations of transitivity in choices; the second class (2.1.2) has to do with the nature of the ideal altemative, and properties in the third class (2.1.3) describe characteristics of probabilistic choice behavior in a unidimensional psychological space.

\subsubsection{Stochastic transitivity}

In deterministic unfolding, transitivity is a property of choice behavior that is necessary for an unfolding representation, as an obvious consequence of the transitivity of distance comparisons. In probabilistic unfolding this transitivity requirement is not abandoned altogether, but relaxed to a probabilistic analogue.

Probabilistic expressions of the transitivity property of rational choice behavior are among the first and most extensively documented properties of probabilistic choice behavior. For historical reasons they are called "stochastic" - not probabilistic - transitivity definitions.

The following five probabilistic versions of the transitivity condition are 
known from the literature. They are expressed as conditions for all elements $w, x, y, s_{1}, s_{2}, \ldots, s_{n}(n>2)$ of the set of potential decision altematives $X$ in a BCP structure $\langle X, p\rangle$

Stochastic Acyclicity (STA)

$\frac{1}{2}<p_{s_{1} s_{2}} \wedge \frac{1}{2}<p_{s_{r^{\prime}},} \wedge \ldots \wedge \frac{1}{2}<p_{s_{s-1} s_{A}} \Rightarrow \frac{1}{2} \leq p_{s_{1} s_{A}}$

Weak Stochastic Transitivity (WST)

$\frac{1}{2} \leq p_{w x} \wedge \frac{1}{2} \leq p_{x y} \Rightarrow \frac{1}{2} \leq p_{w y}$

Moderate Stochastic Transitivity (MST)

$\frac{1}{2} \leq p_{w x} \wedge \frac{1}{2} \leq p_{x y} \Rightarrow \min \left\{p_{w y}, p_{x y}\right\} \leq p_{m y}$

Strong Stochastic Transitivity (SST)

$\frac{1}{2} \leq p_{w x} \wedge \frac{1}{2} \leq p_{x y} \Rightarrow \max \left\{p_{m x}, p_{x y}\right\} \leq p_{m y}$

Strict Stochastic Transitivity (STST)

$\left[\frac{1}{2} \leq p_{w z} \wedge \frac{1}{2} \leq p_{x y} \Rightarrow \max \left\{p_{w x}, p_{x y}\right\} \leq p_{w y}\right] \wedge$

$\left[\frac{1}{2}<p_{w z} \vee \frac{1}{2}<p_{x y} \Rightarrow \max \left\{p_{w x}, p_{x y}\right\}<p_{m y}\right]$.

As far as we know, Fishburn (1973) has been the first to describe stochastic acyclicity (STA), which can be considered to be a probabilistic analogue of the acyclic form of transitivity. Marschak (1960) attributed the second definition (WST) and the fourth (SST) to Valvanis-Vail, who formulated them in 1957 as a result of his work in cooperation with Coombs. The third expression (MST) is attributed to Georgescu-Roegen (1958) and Chipman (1960). The fifth definition (STST) was formulated by Tversky and Russo (1969), and is sometimes referred to as the "strong version of strong stochastic transitivity" (Roberts, 1971) or "strong stochastic transitivity" (Tversky \& Russo, 1969).

All five probabilistic transitivity levels are related to distinct preference relations, defined through choice probabilities, which are each required to be transitive. Some of these preference relations will be elucidated later on in Section 2.1.2. 
Table 2.1

Levels of stochastic transitivity necessarily fulfilled in probabilistic unfolding models

MODELS

STOCHASTIC TRANSITIVITY

STA WST MST SST STST

RANDOM CONFIGUAATION MODELS

Random coordinate models

Case I (double sampling)

Case II (single sampling)

Case III (single sampling - ideal only)

Case IV (alternatives only)

Random distance models

RANDOM RESPONSE MODELS

Strong unfolding models

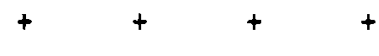

(a)

Midpoint unfolding models

Note - (a) Depends on the cumulative distribution function

These five forms of stochastic transitivity are hierarchical; STA being the lowest and STST the highest form. Any higher form of stochastic transitivity implies all lower ones, as can be inferred from the definitions. For example, let $p_{a b}=.8$ and $p_{b c}=.6$. Stochastic acyclicity then requires $p_{a c} \geq .5$, weak stochastic transitivity implies $p_{a c}>.5$, MST implies $p_{a c} \geq .6$, SST implies $p_{a c} \geq .8$ and STST implies $p_{a c}>.8$. As can be easily inferred from this example, no lower form of stochastic transitivity necessarily implies a higher one.

Several experimental results have led researchers to question the appropriateness of choice theories requiring strong and strict stochastic transitivity, especially when not all alternatives are equally comparable (Coombs, 1958; Chipman, 1958; Krantz, 1967; Tversky \& Russo, 1969; Rumelhart \& Greeno, 1971). Doubts on the validity of the weaker levels of stochastic transitivity are scarce. Theoretical reflections were presented by authors as May (1954), and Morrison (1963). Only Tversky (1969) has reported demonstrations in which even stochastic acyclicity seemed to be violated. 
As could be expected, not all probabilistic unfolding theories imply the same level of stochastic transitivity. For each class of models presented in Chapter 1 , Table 2.1 indicates which levels of stochastic transitivity have to be present in each BCP structure. As in the remainder of this chapter, we will read "random distance models" as models that satisfy the specifications of either Ramsay (1969), Croon (1983), or DeSarbo, De Soete and Eliashberg (1987), unless explicitly stated otherwise. Proofs of the necessity of the respective stochastic transitivity conditions, and all other necessary conditions, can be found in Section 2.2 .

The Coombs-Zinnes-Griggs theory implies that at least moderate stochastic transitivity holds. The assumption of double sampling of the ideal coordinate even implies strict stochastic transitivity. The random distance theories imply strict stochastic transitivity. The BCP structures in a strong unfolding model necessarily satisfy strong stochastic transitivity, and strict stochastic transitivity if the cumulative distribution function in the model is strictly increasing. On the other hand, midpoint unfolding models necessarily satisfy moderate stochastic transitivity only.

As can be concluded from this overview, the required level of stochastic transitivity is rather high for all theories. All theories expect stochastic transitivity to hold at least on the moderate level.

\subsubsection{The ideal point}

The next class of properties are all related to the ideal alternative. Coombs (1958) defined this ideal in unfolding as "a (hypothetical) stimulus which the subject would prefer to all other stimuli". This is a clear definition, if one assumes that preferences are revealed in choices. If so, the ideal will always be chosen from every option set containing it. Despite its apparent clarity, the meaning of this definition is less obvious in terms of probabilistic choice behavior. In order to clarify it we need to know how preference is to be expressed in terms of choice probabilities.

Elsewhere (Bossuyt, 1985) we have explored several preference relations defined through binary choice probabilities, and their implications for probabilistic choice behavior, given Coombs' definition of the ideal. From these we derived four possible "Ideal Point" properties of probabilistic choice behavior, as altemative probabilistic elaborations of Coombs' definition of the ideal alternative. Each of these properties regards probabilistic choice behavior in a paired comparisons task, when one of the available alternatives is the ideal one. 
Relationships between binary preference relations and binary choice probabilities have been studied by quite a number of authors (for a review, see Fishbur, 1977). A simple probability-based binary preference relation is the following:

$$
\begin{aligned}
y D_{1 / 2} x & \Leftrightarrow p_{y x}<p_{x y} \\
& \Leftrightarrow 1 / 2<p_{x y} .
\end{aligned}
$$

The alternative $x$ is preferred to the alternative $y$ if the probability of choosing $x$ out of the pair $\{x, y\}$ exceeds the probability of choosing $y$. If this preference relation $D_{12}$ is adopted, then Coombs' definition implies that no alternative should have a choice probability exceeding one half when the other altemative in the pair of options is the ideal. We will call this condition IP.1:

Ideal Point condition 1 (IP.1)

$p_{\mathrm{xa}} \leq p_{\mathrm{zx}}$.

The relation $D_{1 / 2}$ can be easily extended into a family of preference relations $D_{2}$ (Luce, 1959), by requiring the choice probability to exceed a constant $\lambda$, a point in the half-open real interval $[.5,1)$ :

$$
y D_{\lambda} x \Leftrightarrow \lambda<p_{x y}
$$

This index $\lambda$ can be interpreted as a "level of decisiveness", to be determined by an analyst of probabilistic choice behavior. If $\lambda<p_{x y}$ we say that $x$ is preferred to $y$ on level of decisiveness $\lambda$. This notion of a level of decisiveness is exemplary for a particular interpretation of probabilistic choice behavior: Extreme choice probabilities are assumed to reflect greater ease in making a choice, while probabilities close to one half reflect more difficult choices. If there exists a $\lambda_{1}$ and $a \lambda_{2}$ for which

$$
\begin{aligned}
& y D_{\lambda_{1}} x \wedge y D_{\lambda_{1}} w \\
& y D_{\lambda_{2}} x \wedge \neg y D_{\lambda_{2}} w
\end{aligned}
$$

we say that $x$ is preferred to $y$ on a higher level of decisiveness than $w$ is to $y$.

For each one of these preference relations $D_{\lambda}$, conditions comparable with IP. I can be formulated. If we translate Coombs' description into the postulate that no altemative is preferred to a second on a higher level of decisiveness than the ideal is, we arrive at condition IP.2: 
Ideal Point condition 2 (IP.2)

$p_{\mathrm{yx}} \leq p_{\mathrm{zx}}$.

A more stringent Ideal Point condition states that the ideal is always preferred to all other altematives, for all values of $\lambda$ in the class of relations $D_{\lambda}$ :

Ideal Point condition 3 (IP.3)

$p_{u}=1$.

Condition IP. 3 states that there is no random choice variability if the ideal is one of the available altematives: The ideal will always be chosen. Of the three Ideal Point conditions presented so far, it is obviously the strongest.

To understand the following condition IP.4, which describes the probabilities in a BCP system, we have to consider two alternatives $a$ and $b$, and two subjects, $i, j$ with their corresponding ideals $z_{\imath}, z_{\text {, }}$. Our question is: Which subject will prefer $a$ to $b$ on a higher level of decisiveness? A general answer is that this will obviously depend on the magnitude of the respective ideal-alternative distances. However, the following assumption seems plausible: If $a$ coincides with the ideal for one subject, but not for the other, the second subject will not prefer $a$ to $b$ on a higher level of decisiveness than the first subject will. This is captured in condition IP.4:

Ideal Point condition 4 (IP.4)

$d_{z, x}=0 \wedge d_{z, x} \geq 0 \Rightarrow p_{x y} \leq p_{\Delta x y}$.

Condition IP.4 implies that if for some subject the ideal and an altemative $x$ coincide, no other subject with an ideal different from $x$ will prefer $x$ to some other alternative $y$ on a higher level of decisiveness than the first subject will.

It is very well possible to discuss at length the theoretical plausibility of these four Ideal Point conditions, as could be done for the stochastic transitivity conditions. Yet contrary to the transitivity conditions, none of the Ideal Point conditions has ever been subjected to an evaluation based on empirical data. We ourselves are not inclined to make any a priori statements on their validity. As we announced, the principal aim in formulating these possible properties of probabilistic choice behavior involving perfect or ideal alternatives was to obtain a set of conditions that could be used in comparing probabilistic unfolding theories. 
Table 2.2

Ideal point conditions necessarlly sattsfied in probabilistic unfolding models

MODELS

IDEAL POINT CONDITIONS

$\begin{array}{llllll}\mathbb{P} 1 & \mathbb{P} 2 & \mathbb{P} 3 & \mathbb{P} 4 & \text { UI } & \text { UD }\end{array}$

RANDOM CONFIGURATION MODELS

Random coordinate models

Case I (double sampling)

Case II (single sampling)

Case II (single sampling ideal only)

Case IV (alternatives only)

Random distance models

Ramsay-Croon

DeSarbo c s.

RANDOM RESPONSE MODELS

Strong unfolding models - convex PF

Strong unfolding models - concave PF

Midpoint untolding models

Note - PF preference function, (a) Depends on the preference function

It should come as no surpnse that existing probabilistic unfolding theories do differ on the kind of Ideal Point conditions they require. The results are summanzed in Table 22 . In all models the most elementary condition IP 1 holds. However, condition IP. 2 does not necessarily hold in a number of models In midpoint unfolding models, for example, $p_{y x}$ will exceed $p_{x x}$ if $y$ is more simular to $x$ than $z$ is. Condition IP.3 is always satısfied in Ramsay-Croon random distance models, and in some strong unfolding models. In RamsayCroon random distance models, the vanance of the error distribution degenerates as the errorless value approaches zero. If the errorless value becomes zero, there is no longer any random variability, the probability of confusion tends to zero, and choice probabilities become zero and unity.

Condition IP 4 is not supposed to hold in every probabilıstic unfolding model. In a model of a random coordinate theory, in which the coordinate of the ideal 
is a random variable, it is not required to hold, since the assumed uncertainty in locating alternatives and ideal in the psychological space influences the choice probability, even when the errorless distance between the coordinates of the ideal and one altemative approaches zero. Condition IP.4 holds in random distance models, because error distributions in these theories degenerate when the errorless distance reduces to zero. In a midpoint unfolding model IP.4 may be violated, and in a strong unfolding model, the necessity of this condition depends on the form of the preference function. In a strong unfolding model, IP. 3 and IP.4 will always be satisfied only if the limit of the preference function, as the distance approaches zero, becomes infinity. In a unidimensional strong unfolding model, IP. 4 will be satisfied if and only if the preference function is convex. (A real-valued function $g$ over the nonnegative reals is convex if, for any two $r, t$

$$
g((1-\alpha) r+\alpha t) \leq(1-\alpha) g(r)+\alpha g(t) \quad(\alpha \leq 1) .
$$

holds. A function $g$ is strictly convex if the inequality is strict. A function $g$ is (strictly) concave if and only if $-g$ is (strictly) convex.)

The remaining Ideal Point conditions require the introduction of a new concept, which will be of use in describing unidimensional unfolding applications. An unfolded order is a ranking of the altematives that is monotonic with respect to the ordering of the corresponding coordinates. This means that the altematives $a b c$ are sequenced as $\overrightarrow{a b c}$ in the unfolded order if their (errorless) coordinates can be ordered as $a<b<c$ or $a>b>c$, or, altematively, if $d_{a c}=d_{a b}+d_{b c}$.

The following condition is related to condition IP.4, but it applies in the unidimensional case only. Whenever we have $\overrightarrow{y x z_{\imath} z}$, in the unfolded order, then either the

unilaterally decreasing condition (UD)

$p_{u g} \geq p_{\mu y}$

holds, or the

unilaterally increasing condition (UI)

$p_{u y} \leq p_{\text {pry }}$.

is satisfied. 


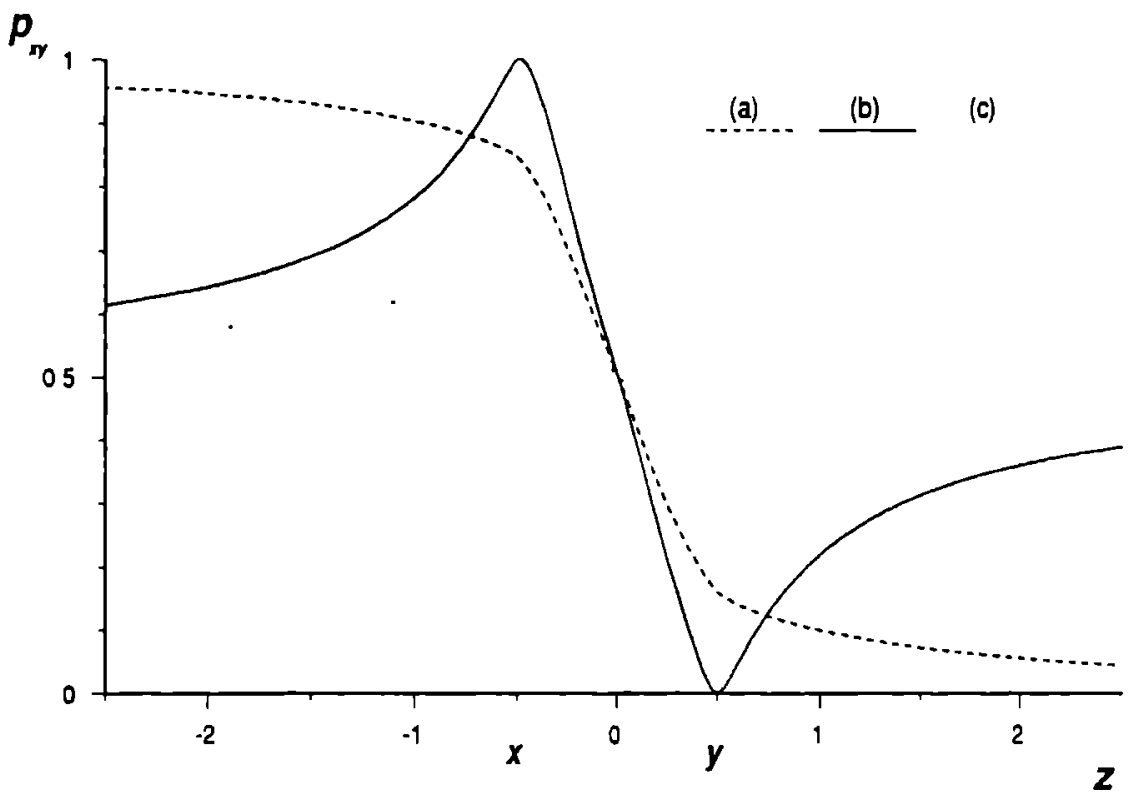

Figure 2.1 - Tracelines for the choice probablity in two unidımensional strong unfolding models (a), (c) and a random distance model (b). Each line indicates the probability of choosing $x$ over $y$, as a function of $z$. Coordinates for $x, y$ are $-.5, .5$ respectively. Tracelines (a) and (c) correspond to strong unfolding models with a standard normal distribution function and a power preference function with $\tau=1.3, \tau=0.3$ respectively. Traceline (b) corresponds to a model of Ramsay's random distance theory.

As Croon (1983) was the first to notice, in some probabilistic unfolding theories the choice probabilities are predicted to become more extreme when the distances to the ideal are jountly increased, whereas in other theones these probabilities approach one half. An example of the former class are the existing random coordinate theories, with the assumption of random ideal coordinates. For altematives more removed from the ideal, choices will be less influenced by the vanability in the location of the ideal coordinate and, hence, will become more extreme. On the other hand, the Ramsay-Croon random distance theories predict the unilaterally decreasing condition. Increasing the errorless idealalternative distances in these models increases the variances of the corresponding error distributions, which in its turn leads to a higher probability of confusion. Whether a strong unfolding theory predicts the decreasing or increasing property, or both, depends entirely on the form of its preference function. A concave decreasing preference function leads to the increasing 


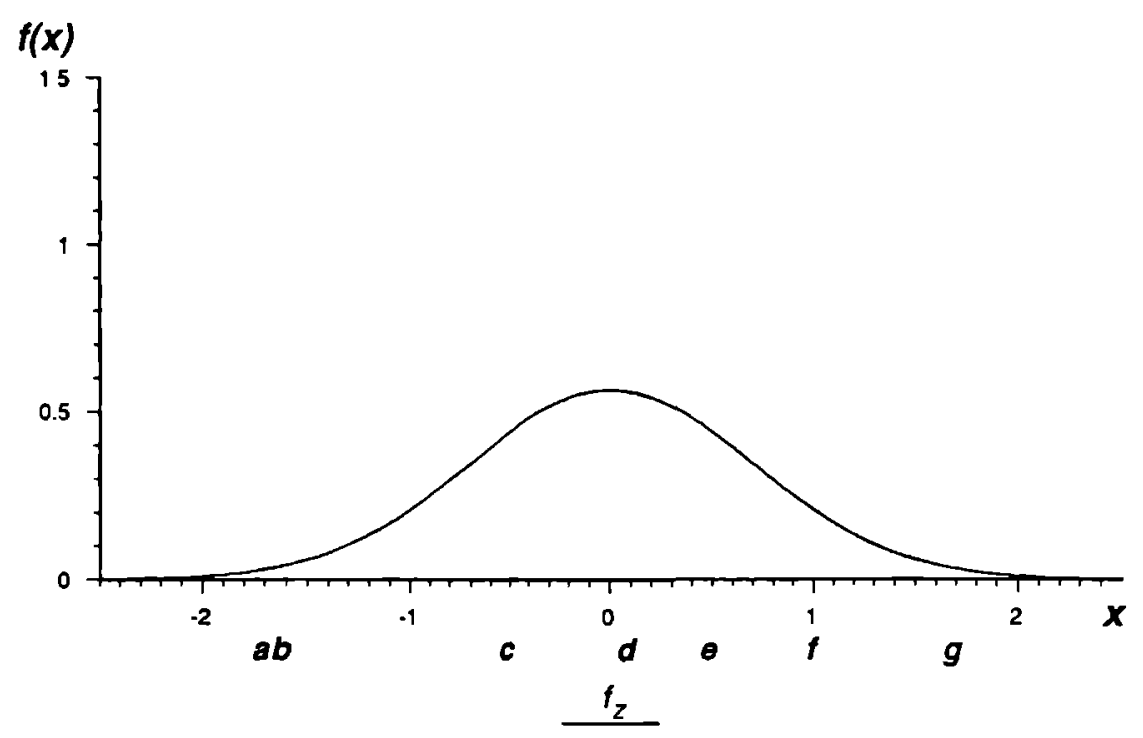

Figure 2.2 - Seven alternatives and an ideal (z), with the probability density function of its coordinate, in a unidimensional space. The coordinate of the ideal has a normal distribution, with expected value 0 and variance .5 .

condition, a convex preference function to the decreasing condition.

In Figure 2.1 choice probabilities are represented that satisfy either the unilaterally increasing or decreasing condition. The choice probability $p_{x y}$ is depicted as a function of the coordinate of the ideal in three different models. In all three models the coordinates of the alternatives are $x=-.5, y=.5$. Tracelines (a) and (c) correspond to strong unfolding models with a standard normal cumulative distribution function and a power preference function (see equation [1.24]), with $\tau=1.3, \tau=0.3$ respectively. Traceline (b) corresponds to a model of Ramsay's random distance theory. The choice probabilities depicted by traceline (a) satisfy the unilaterally increasing condition, whereas the probabilities depicted by the two other tracelines (b) and (c) satisfy the unilaterally decreasing condition. One can also easily infer from this figure that only the probabilities for (b) satisfy the Ideal Point condition IP.3.

Finally, there is a also a bilateral and a symmetry condition:

bilateral condition (BIL)

$$
\begin{aligned}
& \overrightarrow{x z_{1} z_{j} y} \Rightarrow p_{x y} \leq p_{u x} \\
& \text { symmetry condition (SYM) } \\
& x=z_{1} \wedge y=z, \Rightarrow p_{u y}=p_{x x} .
\end{aligned}
$$


Table 2.3

Choice probabilities satisfying characteristic monotonicity

\begin{tabular}{cccccccc}
\hline & $a$ & $b$ & $c$ & $d$ & $e$ & $f$ & $g$ \\
$a$ & 0.500 & 0.010 & 0.060 & 0.129 & 0.198 & 0.310 & 0.500 \\
$b$ & 0.990 & 0.500 & 0.069 & 0.144 & 0.218 & 0.336 & 0.528 \\
$c$ & 0.940 & 0.931 & 0.500 & 0.389 & 0.500 & 0.638 & 0.802 \\
$d$ & 0.871 & 0.856 & 0.611 & 0.500 & 0.664 & 0.782 & 0.898 \\
$e$ & 0.802 & 0.782 & 0.500 & 0.336 & 0.500 & 0.856 & 0.940 \\
$f$ & 0.690 & 0.664 & 0.362 & 0.218 & 0.144 & 0.500 & 0.972 \\
$g$ & 0.500 & 0.472 & 0.198 & 0.102 & 0.060 & 0.028 & 0.500 \\
\hline
\end{tabular}

Note - Row and column indices are arranged in the unfolded order; $d$ is the alternative closest to the ideal $z$; row minumum in italics.

Both conditions are predicted by all probabilistic unfolding theories discussed in Chapter 1. The meaning of the bilateral condition is simple: If two ideals are located in between two altematives $x$ and $y$, the probability of choosing $x$ over $y$ is not higher for the subject located closer to $y$.

\subsubsection{Unidimensional unfolding}

In this section some properties of probabilistic choice behavior in a unidimensional unfolding space will be examined. We will distinguish between three related conditions, that can be defined using the notion of the unfolded order. To arrive at these conditions, three distinct random coordinate assumptions will be used. These will illustrated by a set of seven altematives and an ideal, as shown in Figure 2.2 and Figure 2.3.

\section{(1) Characteristic monotonicity}

The first condition holds in a model of the random ideal coordinate class of random configuration theories. In these theories, all choice inconsistencies are attributed to a random variability in the location of the ideal. We assume only one ideal coordinate value is sampled when making a choice from a pair of altematives.

If we consider the altematives $\{d, f, g\}$ in Figure 2.2, we see that the random ideal coordinate assumption implies that the probability $p_{d f}$ will not exceed $p_{d g}$, and that $p_{d_{g}}$ will not exceed $p_{f_{g}}$. Since the coordinate $f$ is closer than $g$ to the errorless value of the distribution of the ideal coordinate, the possibility of picking a coordinate for the ideal closer to $d$ is higher for a choice between $d$ 


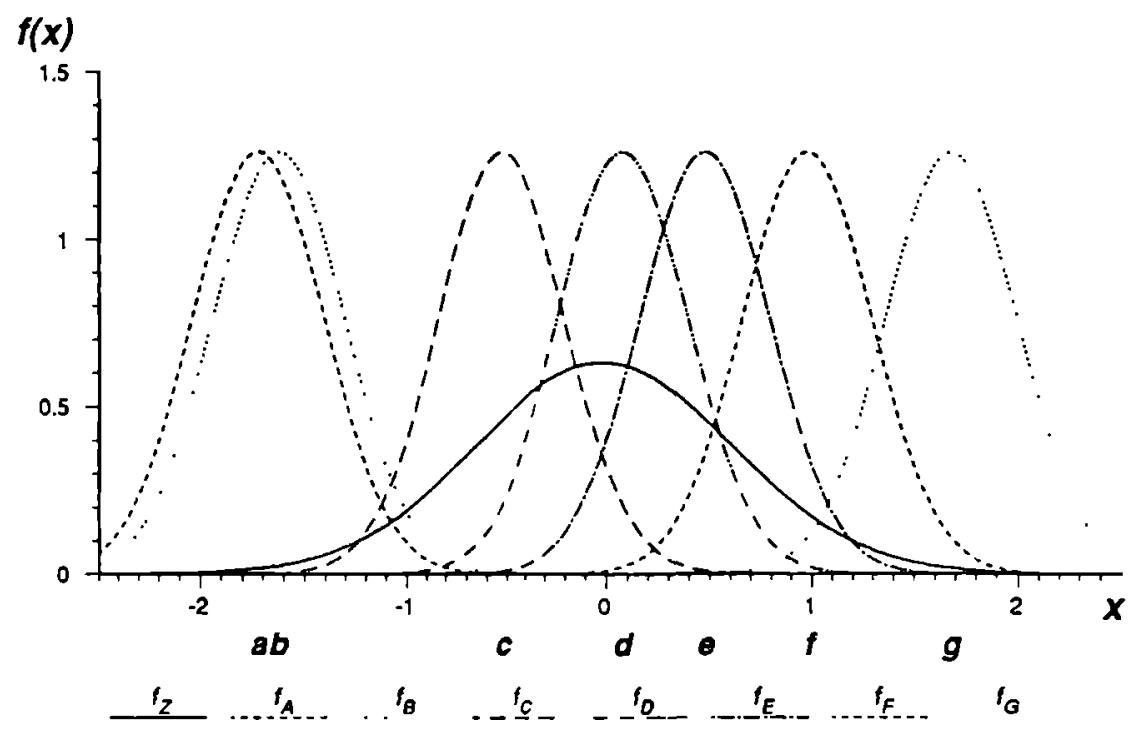

Figure 2.3 - Seven alternatives and an ideal, with the probability density function for their coordinates, in a unidimensional space. The coordinate of the ideal has a normal distribution, with expected value 0 and variance .4. The coordinates of the alternatives have independent normal distributions, with variances equal to 1.

and $g$. This relation between the unfolded order and the choice probabilities is captured in the following condition, which has been called characteristic monotonicity (Dijkstra, van der Eijk, Molenaar, Van Schuur, Stokman \& Verhelst, 1980):

characteristic monotonicity (CM)

$\overrightarrow{w x y} \Rightarrow p_{w z} \leq p_{w y} \wedge p_{m y} \leq p_{x y}$.

In Table 2.3 we have listed binary choice probabilities for the altematives of Example 1 in a random ideal coordinate model, with a normal distribution with a variance of one half for the ideal coordinate. The row and column indices are arranged in the unfolded order. Each entry in this matrix, which is usually called a dominance matrix, contains the probability that the row element is chosen out of a pair consisting of the row and the column element. Upon an inspection of the dominance matrix in Table 2.3, we see that the choice probabilities in each row decrease from the left towards the main diagonal, and increase from the main diagonal to the right. 
Table 2.4

Choice probabilities satisfying bilateral monotonicity

\begin{tabular}{cccccccc}
\hline & $a$ & $b$ & $c$ & $d$ & $\theta$ & $f$ & $g$ \\
$a$ & 0.500 & 0.460 & 0.126 & 0.087 & 0.126 & 0.244 & 0.500 \\
$b$ & 0.540 & 0.500 & 0.149 & 0.105 & 0.149 & 0.276 & 0.540 \\
$c$ & 0.874 & 0.851 & 0.500 & 0.430 & 0.500 & 0.666 & 0.874 \\
$d$ & 0.913 & 0.895 & 0.570 & 0.500 & 0.570 & 0.730 & 0.913 \\
$e$ & 0.874 & 0.851 & 0.500 & 0.430 & 0.500 & 0.666 & 0.874 \\
$f$ & 0.756 & 0.724 & 0.334 & 0.270 & 0.334 & 0.500 & 0.756 \\
$g$ & 0.500 & 0.460 & 0.126 & 0.087 & 0.126 & 0.244 & 0.500 \\
\hline
\end{tabular}

Note - Row and column indices are arranged in the unfolded order; $d$ is the alternative closest to the ideal $z$; row minımum in italics.

Characteristic monotonicity is always found in Zinnes-Griggs models Case III (ideal coordinate only random - single sampling) and in all midpoint unfolding models. Characteristic monotonicity can also be found in ZinnesGriggs models Case $\Pi$ (all coordinate random variables - single sampling), though not necessarily, and is never to be found in a model of one of the remaining theories.

\section{Bilateral monotonicity}

If we assume that the coordinates of the altematives are also random variables, and assume double sampling of the ideal coordinate, we arrive at a different structure, which resembles a model of the Coombs-Zinnes-Griggs theory Case I (see Figure 2.3 for an example). We make the additional assumption that the altemative coordinate distributions have the same distribution, except for their location parameters.

As could be expected, characteristic monotonicity is no longer required in this structure. In fact, for unilateral pairs - pairs of altematives for which the expected values are both either smaller or greater than the ideal coordinate the choice probabilities will be uniquely determined by the amount of overlap between the corresponding coordinate distributions. If we consider once more the triple $\{d, f, g\}$, in Figure 2.3 this time, we see that the shared area under the density distributions for $d$ and $f$ exceeds the corresponding area for $d$ and $g$. For this reason, $p_{d y}$ will not exceed $p_{d g}$. This ordinal relation will always exist, even with different interalternative distances, as long as these three stimuli are organized as $\overrightarrow{z d f g}$ in the unfolded order. Similarly, $p_{f g}$ will not exceed $p_{d_{g}}$. 
We have captured these relations in an implication whose premises do involve the relative location of alternatives w.r.t. the ideal altemative, and whose conclusion, like the one of characteristic monotonicity, consists of ordinal restrictions.

\section{bilateral monotonicity (BM)}

$$
\begin{aligned}
& {\left[\overrightarrow{w x y z} \Rightarrow p_{m z} \geq p_{m y} \wedge p_{m y} \leq p_{x y} \wedge p_{w z} \leq p_{x w} \wedge p_{x y} \leq p_{y x}\right] \wedge} \\
& {\left[\overrightarrow{w x z y} \Rightarrow p_{m y} \leq p_{x y} \wedge p_{m x} \leq p_{m w}\right] .}
\end{aligned}
$$

Table 2.4 contains choice probabilities for a model with the distributions depicted in Figure 2.3. The coordinates of the altematives are independently identically normally distributed, except for their location parameters, with a common variance of .1. The variance of the distribution of the ideal is .4 . We see that the probabilities in each row of this dominance matrix decrease rowwise from left to right, reaching a minimum at the column corresponding to $d$ - the alternative for which the errorless difference with the ideal coordinate is smallest - and increasing thereafter.

Bilateral monotonicity is required by the Zinnes-Griggs theory Case I and Case IV, and by the random distance theories. It is also necessarily satisfied in all strong unfolding models. It will never be found in a midpoint unfolding model, or a model of the Zinnes-Griggs random coordinate theory Case ID. It can be satisfied in a model of the Zinnes-Griggs theory Case $\amalg$, but not necessarily.

\section{Bilateral/characteristic monotonicity}

If we take the same assumptions as in the previous section, considering all coordinates to be random variables, but assume single sampling instead of double sampling, we arrive at a structure that looks like a model of ZinnesGriggs' (1974) theory Case II.

Take again the altematives $\{d, f, g\}$ in Figure 2.3, consider the distributions depicted, but make the assumption of single sampling of the coordinate of $z$. Will $p_{d_{g}}$ exceed $p_{f_{s}}$ or not? The answer to this question is not straightforward, since it depends on the amount of overlap between four distributions: the three distributions of the coordinates of the altematives, and the distribution of the ideal coordinate. If the overlap between the distributions of the coordinates for $f$ and $g$ is very small, and the overlap between the distributions for $d$ and $z$ is large, $p_{d g}$ will not exceed $p_{f g}$. For example, with a variance. 1 for the distributions of the alternatives and .4 for the ideal coordinate, (as in Figure 2.2), we have $p_{d f}=.609, p_{d g}=.910$ and $p_{f_{t}}=.946$. In that case, the choice probabilities will approximately or even perfectly satisfy bilateral monotonicity. 
Choice probabilities satisfying bilateral-characteristic monotonicity.

\begin{tabular}{cccccccc}
\hline & $a$ & $b$ & $c$ & $d$ & $\theta$ & $f$ & $g$ \\
$a$ & 0.500 & 0.413 & 0.054 & 0.117 & 0.186 & 0.301 & 0.500 \\
$b$ & 0.587 & 0.500 & 0.065 & 0.132 & 0.206 & 0.327 & 0.530 \\
$c$ & 0.946 & 0.935 & 0.500 & 0.404 & 0.500 & 0.645 & 0.814 \\
$d$ & 0.883 & 0.868 & 0.596 & 0.500 & 0.609 & 0.781 & 0.910 \\
$\theta$ & 0.814 & 0.754 & 0.500 & 0.391 & 0.500 & 0.771 & 0.946 \\
$f$ & 0.699 & 0.673 & 0.355 & 0.219 & 0.229 & 0.500 & 0.922 \\
$g$ & 0.500 & 0.470 & 0.186 & 0.090 & 0.054 & 0.078 & 0.500 \\
\hline
\end{tabular}

Note - Row and column indices are arranged in the unfolded order; $d$ is the alternative closest to the ideal $z$; row minimum in italics.

On the other hand, if there is a considerable overlap between the distributions for $f$ and $g$, there will be an increased possibility of confusion, rendering $p_{f s}$ less extreme. If so, $p_{d_{g}}$ might exceed $p_{f_{g}}$. For example, if we do not change the location parameters of the distributions in Figure 2.2, but take the variances to be .4 for the altematives and .1 for the ideal, we have $p_{d f}=.572, p_{d g}=.917$ and $p_{f_{g}}=.892$. In that case, the choice probabilities will approximately or even perfectly satisfy characteristic monotonicity.

With the assumptions of single sampling, the choice probabilities can satisfy either bilateral or characteristic monotonicity, or violate both. However, they will always satisfy the following condition:

bilateral/characteristic monotonicity (BCM)

$$
\begin{aligned}
& {\left[\overrightarrow{w x y z} \Rightarrow p_{m y} \geq p_{x y} \wedge p_{w z} \leq p_{x w} \wedge p_{x y} \leq p_{y x}\right] \wedge} \\
& {\left[\overrightarrow{w x z y} \Rightarrow p_{m y} \leq p_{x y} \wedge p_{w x} \leq p_{x w}\right] \wedge} \\
& {\left[\overrightarrow{w w z y} \Rightarrow \neg\left(p_{y x}>p_{y y} \wedge p_{w x}<p_{w y}\right)\right] .}
\end{aligned}
$$

Of the probabilistic unfolding models presented in Chapter 1, models of the Coombs-Zinnes-Griggs Case $I I$ approach are the only ones in which both bilateral or characteristic monotonicity may be violated, yet in which the weaker condition $\mathrm{BCM}$ always holds.

In Table 2.5 we have listed choice probabilities in a model of this ZinnesGriggs theory, Case II, with variances equal to .1 for the distributions of the coordinates, and equal to .4 for the ideal. When we inspect this dominance matrix, the minimum in each row is not necessarily located in column $d$, as in bilateral monotonicity, nor on the main diagonal or in the column next to the 
main diagonal, as in characteristic monotonicity. In the row corresponding to $g$, the lowest probability is in column $e$, instead of in column $f$, (Table 2.3) or $d$ (Table 2.4).

\subsection{PROOFS}

The proofs will be grouped for the three classes of conditions discussed in the previous section.

\subsubsection{Stochastic transitivity}

A few Lemmas will help us in proving most of the theorems on stochastic transitivity for the probabilistic unfolding models we discussed earlier. We start with a definition. A BCP structure $\langle X, p\rangle$ satisfies simple distance decomposability if there exists a cumulative distribution $H$ and a metric $d$ defined over $X \cup\{z\}$ such that for all $x, y \in X$

$$
p_{x y}=H\left(d_{x x}, d_{x y}\right) \text {, }
$$

where $H$ is strictly decreasing in its first argument and strictly increasing in the second. Simple distance decomposability is nothing but the equivalent in unfolding of simple scalability, a property introduced by Krantz (1964). A proof of the following lemma can be found in Tversky \& Russo (1969).

\section{LEMMA 2.1}

Simple distance decomposability implies strict stochastic transitivity.

If we require the function in [2.20] to be just nonincreasing in its first argument, and nondecreasing in the second, we arrive at a weaker form of simple distance decomposability, which we will simply call weak simple distance decomposability.

\section{LEMMA 2.2}

Weak simple distance decomposability implies strong stochastic transitivity, but not necessarily strict stochastic transitivity.

\section{PROOF}

Strong stochastic transitivity (cf. equation [2.4]) is equivalent to the following combination of implications 


$$
\begin{aligned}
& {\left[p_{w x}=.5 \wedge p_{x y}=.5 \Rightarrow p_{w y}=.5\right] \wedge} \\
& {\left[p_{w x}<.5 \wedge p_{x y}<.5 \Rightarrow p_{w x} \leq p_{w y} \wedge p_{x y} \leq p_{m y}\right] .}
\end{aligned}
$$

From the definition of binary choice probabilities follows the equality $H\left(d_{x x}, d_{x x}\right)=.5$. So if the first premise of the first part of [2.21] holds, $H\left(d_{x w}, d_{x x}\right)=H\left(d_{z x}, d_{x x}\right)$ and $d_{x w}=d_{z x}$. Similarly, if the second premise holds, we have $H\left(d_{z x}, d_{x y}\right)=H\left(d_{x y}, d_{x y}\right)$ and $d_{z z}=d_{x y}$. By transitivity, $d_{z w}=d_{x y}$ and $H\left(d_{x w}, d_{x y}\right)=H\left(d_{z y}, d_{z y}\right)=.5$, which proves the implication in the first part in [2.21]. If the first premise of the second implication of condition [2.21] holds, we have $H\left(d_{z w}, d_{z x}\right)>H\left(d_{z x}, d_{z x}\right)$ and $d_{z w}<d_{z x}$. If the second premise holds, $H\left(d_{x}, d_{z y}\right)>H\left(d_{x y}, d_{z y}\right)$ and $d_{z x}<d_{x y}$. The two inequalities in the implication follow, since the distribution function $H$ is nonincreasing in its first argument and nondecreasing in its second. Strict stochastic transitivity may be violated, if $H$ is not strictly decreasing in its first argument or not strictly increasing in its second.

QED

\section{LEMMA 2.3}

If a moderate unfolding model exists, each $B C P$ structure satisfies moderate, but not necessarily strong stochastic transitivity.

\section{PROOF}

The proof relies on results obtained by Halff (1976). Define $\gamma$ as follows

$$
\gamma=\min \left\{\frac{f\left(d_{z w}\right)-f\left(d_{z u}\right)}{g\left(d_{w z}\right)}, \frac{f\left(d_{z x}\right)-f\left(d_{z y}\right)}{g\left(d_{x y}\right)}\right\}
$$

and obtain

$$
\begin{aligned}
& \gamma g\left(d_{w z}\right) \leq f\left(d_{x w}\right)-f\left(d_{x z}\right) \\
& \gamma g\left(d_{x y}\right) \leq f\left(d_{z z}\right)-f\left(d_{z y}\right) .
\end{aligned}
$$

By summing both inequalities, using the triangle inequality $-d_{w y} \leq d_{w x}+d_{x y}$ - and the fact that $g$ is concave by definition (cf. Section 1.2.4) $-g\left(d_{w g}\right) \leq g\left(d_{w z}\right)+g\left(d_{x y}\right)$ - we obtain 


$$
\begin{aligned}
\gamma g\left(d_{w y}\right) & \leq f\left(d_{z w}\right)-f\left(d_{z y}\right) \\
\gamma & \leq \frac{f\left(d_{z w}\right)-f\left(d_{z y}\right)}{g\left(d_{w y}\right)},
\end{aligned}
$$

from which the implication of moderate stochastic transitivity follows.

The violation of strong stochastic transitivity can be easily demonstrated by a counterexample, e.g. $d_{x w}=1, d_{x x}=2, d_{x y}=3, d_{w x}=d_{x y}=1, f(x)=-x^{2}, g(x)=x$. QED

\section{LEMMA 2.4}

In a Zinnes-Griggs model Case I (double sampling of the ideal coordinate), each BCP structure satisfies simple distance decomposability.

\section{LEMMA 2.5}

In a Zinnes-Griggs model Case $V$ (coordinates only), each $B C P$ structure satisfies simple distance decomposability.

As we saw in Section 1.2.1, the choice probabilities in a Zinnes-Griggs model are functionally related to the model's parameters through a doubly noncentral $F$ distribution function. The lemma follows from the relation between the cumulative doubly noncentral $F$ distribution function and its noncentrality parameters. Bezembinder and Bossuyt (1989) described this proof more extensively.

\section{LEMMA 2.6}

In a random distance model, each BCP structure satisfies simple distance decomposability.

This also follows easily from the expressions for the probabilities, as they have been derived in Chapter 1 , combined with the strictly increasing nature of the normal and logistic cumulative distribution functions.

\section{LEMMA 2.7}

In a strong unfolding model each BCP structure satisfies weak simple distance decomposability.

Lemma 2.7 follows from the definition of a strong unfolding model (cf. 1.2.3).

LEMMA 2.8

In a unidimensional Zinnes-Griggs model, the following relation holds: 


$$
d_{x} \leq d_{x y} \Leftrightarrow p_{y x} \leq p_{x y}
$$

\section{PROOF}

Equation [2.25] can be broken down into two equivalencies:

(1) $d_{x x}=d_{x y} \Leftrightarrow p_{x y}=p_{y x}$ and

(2) $d_{x x}<d_{x y} \Leftrightarrow p_{y x}<p_{x y}$.

(1) Suppose $d_{z x}=d_{x y}$ and $p_{x y} \neq p_{y x}$. Using equation [1.6], the equality $\alpha_{z x y}=\alpha_{x y x}$ and $\beta_{x y}=-\beta_{y x}$ (cf. equations [1.7] and [1.8]), we derive from $p_{x y} \neq p_{y z}$ the following inequality:

$$
\left[\Phi\left(\alpha_{z x}\right)-.5\right]\left[\Phi\left(\beta_{x y}\right)-.5\right] \neq 0
$$

This inequality holds if both $\alpha_{u y} \neq 0$ and $\beta_{x y} \neq 0$. Now since $d_{u}=d_{z y}$, either $\alpha_{u y}$ or $\beta_{x y}$ has to be zero, so [2.26] cannot be true, and (1) has to hold.

(2) Suppose $d_{x x}<d_{x y}$ and $p_{x y}<p_{y x}$. Using equation [1.6] once more, we derive from $p_{x y}<p_{y z}$ the following inequality:

$$
\left[\Phi\left(\alpha_{2 x y}\right)-.5\right]\left[\Phi\left(\beta_{x y}\right)-.5\right] \leq 0
$$

Since $d_{z x}<d_{x y}, \alpha_{z y}$ and $\beta_{x y}$ are either both positive or both negative, [2.27] cannot be true, and (2) has to hold.

QED

\section{LEMMA 2.9}

If a unidimensional Zinnes-Griggs model exists, then for every alternative $y$ there exists a point $u$, with $\overrightarrow{z u y}$, such that for every two alternatives $w$ and $x$ with nonidentical coordinates, each distinct from $y$

$$
\left[\overrightarrow{w x u} \Rightarrow p_{w y}<p_{w x}\right] \wedge\left[\overrightarrow{u w x} \Rightarrow p_{m,}>p_{w x}\right] .
$$

\section{PROOF}

We have to prove that $p_{r y}$ as a function of $x$ increases up to the point $u$, and decreases afterwards. The proof follows from the behavior of the partial derivative of $G(z, x, y, \rho)$ in equation [1.6] w.r.t. the coordinate of $x$ : 


$$
\begin{aligned}
& \frac{\partial p_{x y}}{\partial x}=\frac{\phi\left(\alpha_{u y}\right)}{\sqrt{1+\rho_{T_{u x} T_{z y}}}}\left[1-2 \Phi\left(\beta_{r y}\right)\right]-\frac{\phi\left(\beta_{x y}\right)}{\sqrt{1-\rho_{T_{u} T_{\eta}}}}\left[1-2 \Phi\left(\alpha_{2 x}\right)\right] \quad\left(\text { for } \rho_{\tau_{u x} T_{r y}}<1\right) \\
& \frac{\partial p_{x y}}{\partial x}=\frac{\phi\left(\alpha_{u y}\right)}{\sqrt{2}} \theta(y-x) \quad\left(\text { for } \rho_{\tau_{u} \tau_{r y}}=1\right)
\end{aligned}
$$

This partial derivative exists everywhere for $x \neq y$. Assume that $z<y$. We will distinguish between three situations, which exhaustively cover all possibilities:
(1) $m_{x y} \leq z \wedge x<y$,
(2) $z<m_{x y} \wedge x \leq y$
(3) $z<m_{x} \wedge y<x$.

In case (1), $\alpha_{2 y}$ is nonnegative and $\beta_{x y}$ is negative, so the derivative in [2.29] is positive. In case (3), $\alpha_{z y}$ is negative and $\beta_{x y}$ is positive, so [2.29] is negative. In case (2), both $\alpha_{2 x y}$ and $\beta_{x y}$ are negative. If $\rho$ equals unity, the derivative in this interval is positive. For $\rho<1$, the derivative is negative for $x=y$, (in that case $\beta_{x y}$ is zero) and positive for $x<y$ (Case (2)). The derivative reaches a maximum at a point $u$, for which [2.29], with $x=u$, is zero. To show that this point is unique, take $x<u$. In that case, $\alpha_{z x y}>\alpha_{z u y}$ and $\beta_{x y}<\beta_{u y}$, so [2.29] has to be positive. Take $x>u$. In that case, $\alpha_{z x y}<\alpha_{z y y}$ and $\beta_{x y}<\beta_{u y}$, and [2.29] will be negative. The proof for $z \geq y$ can be handled in a similar way.

QED

We can now prove the following theorems.

THEOREM 2.1

In a Zinnes-Griggs random coordinate model Case $I$, each $B C P$ structure satisfies strict stochastic transitivity.

THEOREM 2.2

In a Zinnes-Griggs random coordinate model Case III, each BCP structure satisfies moderate stochastic transitivity; strong stochastic transitivity can be violated.

THEOREM 2.3

In a Zinnes-Griggs random coordinate model Case $I V$, each $B C P$ structure satisfies strict stochastic transitivity.

THEOREM 2.4

In a Ramsay-Croon random distance model, each $B C P$ structure satisfies strict stochastic transitivity. 


\section{THEOREM 2.5}

In a strong unfolding model, each BCP structure satisfies strong stochastic transitivity; strict stochastic transitivity can be violated. THEOREM 2.6

In a midpoint unfolding model, each BCP structure satisfies moderate stochastic transitivity; strong stochastic transitivity can be violated. THEOREM 2.7

In a Zinnes-Griggs model with a unidimensional space, each BCP structure satisfies at least moderate stochastic transitivity.

Theorems 2.1 and 2.3 can be proved using Lemma 2.1, and 2.4 and 2.5 respectively. Theorem 2.4 can be proved using Lemma 2.1 and Lemma 2.6. Theorem 2.5 can be proved using Lemma 2.2 and Lemma 2.7; the violation of STST can occurr if the cumulative distribution function is not strictly increasing. Theorem 2.6 can be proved using Lemma 2.3 and the result that a midpoint unfolding model is equivalent with a moderate unfolding model (cf. Section 1.2.4). Theorem 2.2 can be proved using the result that a Zinnes-Griggs random coordinate model Case III is equivalent with a midpoint unfolding model, and Theorem 2.6. A proof for Theorem 2.7 follows.

\section{PROOF OF THEOREM 2.7}

Take a triple for which the premises of moderate stochastic transitivity are fulfilled: $w, x, y$ with $p_{w x}>.5$ and $p_{x y}>.5$. Four distinct unfolded orders are possible:

(1) $\overrightarrow{w z x y}$ (bilateral below)

(2) $\overrightarrow{z w x y}$ (unilateral)

(3) $\overrightarrow{y w z x}$ (bilateral split) and

(4) $\overrightarrow{y z w x}$ (bilateral above).

Using Lemma 2.8 and Lemma 2.9, it can be shown that in case (1) and (2), $p_{w x}<p_{m y}$ and in case (3) and (4), $p_{m y}>p_{x y}$, which delivers the implication of moderate stochastic transitivity.

QED

\subsubsection{The ideal point}

Again we start with a number of Lemmas. 
LEMMA 2.10

If a BCP structure satisfies weak simple distance decomposability, it satisfies IP.1 and IP.2.

This needs no proof.

LEMMA 2.11

In a unidimensional Zinnes-Griggs model, $p_{n y}$ increases in $z$ when $y<x$.

\section{PROOF}

The partial derivative of the function in [1.6] and [1.16] with respect to $z$ is equal to:

$$
\begin{array}{ll}
\frac{\partial p_{x y}}{\partial z}=\frac{\phi\left(\alpha_{z y}\right)}{\sqrt{1+\rho_{T_{u} T_{z y}}}}\left[2 \Phi\left(\beta_{x y}\right)-1\right] & \left(\text { for } \rho_{T_{u x} T_{z y}}<1\right) \\
\frac{\partial p_{x y}}{\partial z}=\phi\left(\sqrt{2}\left(z-m_{x y}\right)\right) \theta(x-y) & \left(\text { for } \rho_{T_{u x} T_{z y}}=1\right) .
\end{array}
$$

From $y<x$, it follows that $\beta_{x y}$ and $\theta(x-y)$ are positive, so the derivatives in [2.30] are positive.

QED

\section{LEMMA 2.12}

In a midpoint unfolding model, $p_{x y}$ is not decreasing in $z$ when $y<x$.

In a midpoint unfolding model, $p_{x y}$ is not increasing in $x$ when $y<x$.

\section{PROOF}

The partial derivatives of the function in [1.29] with respect to $z$ and with respect to $x$ when $y<x$ are equal to

$$
\begin{aligned}
& \frac{\partial p_{x y}}{\partial z}=h\left(z-m_{x y}\right) \\
& \frac{\partial p_{x y}}{\partial x}=-\frac{h\left(z-m_{x y}\right)}{2}
\end{aligned}
$$


in which $h$ stands for the probability density function corresponding to the cumulative distribution function $H$ in equation [1.29]. The first partial derivative is positive, the second negative.

QED

THEOREM 2.8

In a unidimensional Zinnes-Griggs model IP.I is satisfied in each BCP structure, but IP.3 is not.

THEOREM 2.9

In a unidimensional Zinnes-Griggs model Case I or Case IV, IP.2 is satisfied in each $B C P$ structure.

THEOREM 2.10

In aRamsay-Croon random distance modelIP.1 and IP.2 are satisfied in each $B C P$ structure.

THEOREM 2.11

In a strong unfolding model IP.I and IP.2 are satisfied in each BCP structure.

THEOREM 2.12

In a midpoint unfolding model IP.I is satisfied in each BCP structure, but IP.2 is not necessarily.

THEOREM 2.13

In a unidimensional Zinnes-Griggs model Case II or Case III, IP.2 is not necessarily satisfied.

Theorem 2.8 can be proved with Lemmas $2.8,2.9$. Theorems $2.9,2.10$ and 2.11 follow from Lemma 2.10, and Lemma 2.4 and 2.5, 2.6 and 2.7 respectively. The first part of Theorem 2.12 follows from the definition of a midpoint unfolding model, the second part can be proved with the help of Lemma 2.12. Theorem 2.13 follows from Lemma 2.9: The partial derivative of $p_{x y}$ w.r.t. $x$ is positive for $x=z$ when $\rho>0$.

THEOREM 2.14

In a unidimensional Zinnes-Griggs model, the unilaterally increasing condition is satisfied, but not necessarily IP.4.

THEOREM 2.15

In a midpoint unfolding model, the UI condition is satisfied, but not necessarily IP.4.

THEOREM 2.16

In a strong unfolding model, IP.3 is necessarily satisfied if and only if the limit of the preference function, as the ideal-alternative distance approaches zero, is infinity. 
THEOREM 2.17

In a strong unfolding model, IP.4 is necessarily satisfied if and only if the preference function is convex.

\section{THEOREM 2.18}

In a strong unfolding model, the UI condition is satisfied if the preference function is concave; the UD condition is satisfied if the preference function is convex.

THEOREM 2.19

Conditions IP.3 and IP.4 are satisfied in a random distance model if it satisfies the Ramsay-Croon description and violated if it satisfies the DeSarbo c.s. description.

THEOREM 2.20

The unilaterally decreasing is satisfied in a random distance model if it satisfies the Ramsay-Croon description; the unilaterally decreasing is satisfied if it satisfies the DeSarbo c.s. description.

Theorem 2.14 can be derived from Lemma 2.11, and Theorem 2.15 from Lemma 2.12. If the condition in Theorem 2.16 is not fulfilled, $p_{x z}$ will exceed zero. Theorem 2.19 and 2.20 follow from $2.16-17$ and 2.19 respectively. A Ramsay-Croon random distance model is equivalent with a strong unfolding model with a logarithmic preference function, and a DeSarbo random distance model is equivalent with a strong unfolding model with a squared preference function. A proof for Theorem 2.17 and 2.18 follows.

\section{PROOF OF THEOREM 2.17 AND 2.18}

The implication in condition IP.4 is true in a strong unfolding model whenever for a pair of alternatives $x, y$ and a pair of ideals $z_{t}, z$,

$$
d_{x, y} \leq d_{x_{j} z} \vee d_{x, y} \leq d_{x, y}
$$

is true, so we will just have to consider situations in which [2.33] is false. Define

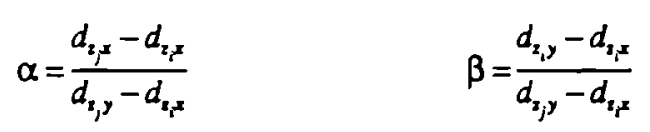

then

$$
\begin{aligned}
& d_{z, y}=(1-\alpha) d_{1, x}+\alpha d_{x, y} \\
& d_{z, y}=(1-\beta) d_{x_{y, x}}+\beta d_{z, y} .
\end{aligned}
$$


If $f$ is convex, we can derive from [2.35] the following inequality:

$$
f\left(d_{x, \beta}\right)+f\left(d_{x_{y}, y}\right) \leq[2-(\alpha+\beta)] f\left(d_{x, y}\right)+(\alpha+\beta) f\left(d_{x, y}\right) .
$$

Since $d_{x_{i}}=0$ and $d_{x, y}=d_{x y}$ (premise of IP.4), we can use the triangle inequality to deduce $1 \leq \alpha+\beta$. From $d_{t, z}<d_{z, y}$ and $f$ nonincreasing, we derive

$$
\begin{array}{r}
{[(\alpha+\beta)-1] f\left(d_{z, y}\right) \leq[(\alpha+\beta)-1] f\left(d_{z_{1} x}\right)} \\
{[2-(\alpha+\beta)] f\left(d_{z_{1},}\right)+(\alpha+\beta) f\left(d_{z_{y} y}\right) \leq f\left(d_{z_{r}}\right)+f\left(d_{z_{y} y}\right) .}
\end{array}
$$

From [2.36] and [2.37] we derive

$$
\begin{gathered}
f\left(d_{z, y}\right)+f\left(d_{x, y}\right) \leq f\left(d_{z, x}\right)+f\left(d_{z, y}\right) \\
H\left(f\left(d_{z, y}\right)-f\left(d_{z, y}\right)\right) \leq H\left(f\left(d_{z, y}\right)-f\left(d_{z, y}\right)\right)
\end{gathered}
$$

(because $H$ is nondecreasing) which delivers the implication of condition IP.4.

To show that a concave preference function may lead to a violation of IP.4, and to deliver a proof for Theorem 2.19, take the premise of the unilateral conditions [2.13] and [2.14]: a unidimensional space with an unfolded order $\overrightarrow{y x z, z}$. In that case, $\alpha+\beta=1$. If the preference function is convax, the inequality [2.38] can be derived immediately from [2.36]. If the preference function is concave, then

$$
\begin{aligned}
& f\left(d_{z, y}\right) \geq(1-\alpha) f\left(d_{z, y}\right)+\alpha f\left(d_{z, y}\right) \\
& f\left(d_{t, y}\right) \geq(1-\beta) f\left(d_{z_{i}}\right)+\beta f\left(d_{z, y}\right) .
\end{aligned}
$$

Summing, rearranging, and applying $H$ leads to

$$
\begin{aligned}
& f\left(d_{z, y}\right)+f\left(d_{z_{1}, y}\right) \geq f\left(d_{z_{1}, z}\right)+f\left(d_{z_{j}, y}\right) \\
& H\left(f\left(d_{z, y}\right)-f\left(d_{z, y}\right)\right) \geq H\left(f\left(d_{t, z}\right)-f\left(d_{x, y}\right)\right)
\end{aligned}
$$


which delivers the unilaterally increasing condition, but may violate IP.4 if $d_{i, x}=0$.

QED

\subsubsection{Unidimensional unfolding}

We need just one more lemma.

LEMMA 2.13

If a unidimensional probabilistic unfolding model exists and each $B C P$ structure in the $B C P$ system satisfies weak simple distance decomposability, then this $B C P$ structure satisfies bilateral monotonicity.

We can now prove the following Theorems:

\section{THEOREM 2.21}

In a unidimensional Zinnes-Griggs model Case I or Case IV, each $B C P$ structure satisfies bilateral monotonicity.

THEOREM 2.22

In a unidimensional random distance model, each BCP structure satisfies bilateral monotonicity.

THEOREM 2.23

In a unidimensional strong unfolding model, each BCP structure satisfies bilateral monotonicity.

THEOREM 2.24

In a midpoint unfolding model, each BCP structure satisfies characteristic monotonicity.

THEOREM 2.25

In a unidimensional Zinnes-Griggs model Case III, each BCP structure satisfies characteristic monotonicity.

THEOREM 2.26

In a unidimensional Zinnes-Griggs model, each BCP structure satisfies bilateral/characteristic monotonicity.

Theorem 2.21 to 2.23 follow from Lemma 2.13, and Lemma 2.4 and 2.5, 2.6, and 2.7 respectively. Theorem 2.24 can be proved using Lemma 2.12 . Theorem 2.25 follows, since a unidimensional Zinnes-Griggs model Case III is equivalent with a midpoint unfolding model. Theorem 2.26 follows immediately from Lemma 2.9. 



\section{probabilistic unfolding theories}

In this section we will deal with the problem of how probabilistic unfolding theories can be evaluated. The approach that will be advocated has been hinted at in Chapter 2, in which properties of probabilistic choice behavior were explored. An examination of the properties of probabilistic choice behavior that are necessarily implied by a probabilistic choice theory resulted in a second classification of existing probabilistic unfolding theories. Looking at it from a different angle, one can consider the assumption that a particular property is satisfied in probabilities of choosing altematives as axioms of alternative theories on probabilistic choice (Type I). Contrary to the theories presented in the Chapter 1 (Type II), these Type I theories are built then on assumed regularities in probabilistic choice behavior, instead of on some cognitive structure of a presupposed form, though the latter may (indirectly) lead to the same sort of regularities.

Examining equivalencies between Type I theories and Type II theories between axioms on properties of choice behavior and axioms on cognitive structures - can lead to a better understanding of the relationships between these categories of theories. For example, it is very well possible that two Type II theories, differing in their basic, structural and conceptual approach to choice behavior, are nevertheless highly similar in the kind of probabilistic choice behavior they try to account for, in terms of the properties they expect to hold. These equivalencies may also prove to be of worth in an evaluation of the relation between theories and observations on choice behavior.

The first section of this chapter contains a description of such an evaluation strategy, within the context of theories of probabilistic choice behavior in general. It provides also a description of basic concepts, such as "model" and "theory", in order to be able to be more precise in describing what is implied by a "test of a model". The section is based on a paper by Bossuyt \& Roskam on the subject $(1987 \mathrm{~b}, 1989 \mathrm{~b})$. The second part of this section contains a discussion on relevant topics in data collection designs and subject sampling schemes. 
Probabilistic choice theory, and with it, the use of probabilistic choice models, originated from the psychophysical laboratories. The primary aim was the assignment of numbers to altematives in a way that could be theoretically defended. Gradually, the ideas became intertwined with notions from classic algebraic utility theory and axiomatic measurement theory. During the past decades we have witnessed a further development of probabilistic choice theory in psychology, largely separated from a growing field of behavioral choice studies. On the other hand, some of the basic ideas have been taken up by economists. The latter has resulted in a research area known as (probabilistic) discrete-choice modelling.

Though both fields now exist for a number of years, anyone trying to get acquainted with the literature is hindered by a surprising lack of unanimity in the terminology, especially when it comes to the use of words as "theory" or "model". As a consequence, it will seldom be immediately clear what is meant if an author writes that a "model is tested".

We were faced with this difficulty when we tried to evaluate members of a numerous subclass of probabilistic choice theories, known as "probabilistic unfolding theories". In order to define what was actually at stake in this evaluation, we felt ourselves forced to construct a conceptual framework with a clear, distinct meaning for "model" and "test of a model", and within which the relation between theory and observations could be adequately described and evaluated. A sketch of this framework is presented in this section. We do not present an overview of probabilistic unfolding, for which the reader is referred to the first chapter. In fact, the theories quoted in this paper only serve an exemplary purpose. They are discussed within their axiomatized form, with a set and a binary choice probability function as variables, the familiar settheoretical and logical constants, and the usual predicates and operations on the reals.

Within the probabilistic choice theories we will make a somewhat uncommon distinction between Type I and Type II theories. Contrary to Type I theories, Type II theories presume the existence of real-valued functions over the set of alternatives, to which the choice probabilities can be functionally related. Close to its meaning in logic, a possible realization of a theory will be an appropriate settheoretical structure. For a theory on probabilistic choice, this will be an ordered couple, consisting of a particular set of alternatives and a binary choice probability function. A possible realization of a theory will be called a model of this theory if it satisfies all its valid sentences: its axioms and all sentences that can be logically derived from them. 
Within our view, a model of a theory cannot be a model of the data, for the simple reason that probabilities are in general not part of recorded data, which means they cannot be derived from observations through a coding or a classification procedure. At most they can be estimated, but never observed. Corresponding to the idea of a possible realization of a theory, we therefore use, following Suppes (1962), the notion of a possible realization of the data. This also is a settheoretical structure of the appropriate type, containing all the information needed to test the theory in question. A possible realization of the data will be called a model of the data if the information it contains is valid.

The relation between Type II theories on probabilistic choice and observations is usually evaluated through a statistical approach. In this section, we will defend the thesis that this relation should more frequently be evaluated through an evaluation of the relation between observations and Type I theories. This relation can be evaluated through a statistical approach that is similar to the one used for evaluating the goodness of fit between models of data and models of Type II theories. The difference between the first, or direct strategy (model of data - model of Type $\Pi$ theory) and the second, or indirect strategy (model of data - model of Type I theory - Type II theory) parallels the distinction between "scaling" and the evaluation of necessary and sufficient axioms for a homomorphic mapping of an algebraical relational system on a numerical relational system in axiomatic measurement theory (Krantz, Luce, Suppes and Tversky, 1971, pp. 32-33).

\subsubsection{Models of theory}

To ease the exposition, we will restrict ourselves to paired comparisons, and one choosing subject, which can either be a single person or a group of persons. In a paired comparisons task, a researcher wants to gather information on choices from a nonempty set of feasible alternatives. Out of this set, option sets of two elements each are constructed. From each option set, the subject has to choose one and only one element, the no-choice option being eliminated.

A probabilistic choice theory views a choice from such an option set probabilistically. A choice is regarded as the realization of an independent Bernoulli trial, with a particular probability of choosing the first alternative. This choice probability is supposed to remain constant over repeated presentations of the same option set.

A possible realization of this general theory of probabilistic (binary) choice is an ordered couple $\langle X, p\rangle$, satisfying the following axioms:

BCP.1 $X$ is a nonempty set

BCP.2 $p$ is a real-valued function defined on $X \times X$ as follows: 


$$
\begin{array}{ll}
\forall x, y \in X: & \text { a. } 0 \leq p_{n} \leq 1 \\
& \text { b. } p_{n}+p_{m}=1 \\
& \text { c. } p_{m}=\frac{1}{2} .
\end{array}
$$

We will call such a couple $\langle X, p\rangle$ a binary choice probability (BCP) structure, and read $p_{x y}$ as "the probability that the altemative $x$ is chosen out of an option set consisting of $x$ and $y$ only".

Besides these mathematical constraints a theory on probabilistic choice assumes some additional form of consistency in the choice probabilities. We will distinguish between two classes of theories, which we will introduce with some simple examples.

\section{(1) Theories on probabilistic choice - Type I}

In a first class of theories the consistency is imposed through a number of constraints on the choice probabilities. A simple theory capturing this consistency assumption is the "weak stochastic transitivity" theory $T_{1}$ defined through the following axiom A.1:

$$
\begin{aligned}
& \text { АХІом A.1 } \\
& \forall w, x, y \in X: \quad \frac{1}{2} \leq p_{w x} \wedge \frac{1}{2} \leq p_{x y} \Rightarrow \frac{1}{2} \leq p_{m y} .
\end{aligned}
$$

Loosely interpreted, this axiom expresses the notion that if a subject is inclined to choose $w$ out of $\{w, x\}$ and $x$ out of $\{x, y\}$, this subject will also be inclined to choose $w$ out of $\{w, y\}$. In a way, weak stochastic transitivity is the simplest probabilistic analogue of the rational transitivity prescription in algebraic utility theory.

A second theory, which we will the "strong stochastic transitivity theory" $T_{2}$, is defined through axiom A.2:

$$
\begin{aligned}
& \text { АХІОМ A.2 } \\
& \forall w, x, y \in X: \quad \frac{1}{2} \leq p_{w x} \wedge \frac{1}{2} \leq p_{x y} \Rightarrow \max \left\{p_{w x}, p_{x y}\right\} \leq p_{m y} .
\end{aligned}
$$

This axiom implies that whenever a subject is inclined to choose $w$ rather than $x$ out of $\{w, x\}$, the probability of choosing $w$ instead of some third element $y$ from any other option set will exceed the probability of choosing $x$ out of $\{x, y\}$ (for a proof, see Tversky \& Russo, 1969). 
To complete our set of examples of Type I theories, we present theory $T_{3}$. It is defined through axiom A.3, which states that the probabilities of any two intransitive choice cycles in a subset of three alternatives are identical:

\section{Aхाом A.3}

$\forall w, x, y \in X: \quad p_{m x} p_{x y} p_{y w}=p_{w y} p_{y x} p_{n w}$

So far we have three examples of Type I theories of probabilistic choice. In each theory a distinct form of consistency in the choice probabilities is assumed to exist, either through ordinal constraints (A.1, A.2) or through an equality restriction (A.3).

For any of the theories on probabilistic choice, a possible realization (i.e., a BCP structure) will be called a model of a theory $T_{4}$ if it contains no violations of the corresponding axiom $\mathrm{A}_{\mathrm{i}}$. So, given a BCP structure, testing whether or not it is a model of a particular theory is a straightforward task: One simply checks the relevant axioms. As an example, consider the BCP structure $\langle E, p\rangle$

$$
\begin{aligned}
& E=\{e, f, g\} \\
& p_{e f}=3 / 5, \quad p_{f g}=2 / 3, \quad p_{e g}=7 / 10 .
\end{aligned}
$$

It is easy to verify that this BCP structure satisfies weak stochastic transitivity (A.1) and is therefore a model of $T_{1}$. It satisfies strong stochastic transitivity (A.2) and is a model of $T_{2}$, but it fails to satisfy A.3, so it cannot be a realization of theory $T_{3}$ and, hence, fails to be a model of this theory.

The three theories presented are hierarchically related to one another. Strong stochastic transitivity implies weak stochastic transitivity, so any model of $T_{2}$ will also be a model of $T_{1}$. The same relation holds between theories $T_{2}$ and $T_{3}$ : Axiom A. 3 implies A.2. As a consequence, if a BCP structure fails to be a model of $T_{2}$, it cannot be a model of $T_{3}$.

\section{Theories on probabilistic choice - Type II}

Whereas in the Type I theories the consistency was imposed through constraints on the choice probabilities themselves, the Type II theories state the existence of a real-valued function over the set of alternatives $X$ (or, alternatively, its product set), to which the choice probabilities are related. Usually these functions are interpreted as utility functions. We present three simple examples.

The first is the weak utility theory $T_{4}$. A BCP structure $\langle X, p\rangle$ is a weak utility model if there exists a real-valued function $u$ defined on $X$ such that 
AxIOM A.4

$\forall x, y \in X: \quad p_{x y} \geq p_{y x} \Leftrightarrow u(x) \geq u(y)$.

A second example is the Fechnerian or strong utility theory $T_{5 .}$ A strong utility model is a BCP structure $\langle X, p\rangle$ for which there exist a cumulative distribution function $H$ and a utility function $u$ defined over $X$ such that

AXIOM A.5

$\forall x, y \in X: \quad p_{x y}=H[u(x)-u(y)]$.

As a third example we present the strict utility theory $T_{6}$. A strict utility model is a BCP structure $\langle X, p\rangle$ for which there exists a utility function $u$ defined over $X$ such that

AхIOM A. 6

$\forall x, y \in X: \quad p_{x y}=\frac{u(x)}{u(x)+u(y)}$.

How can we test whether or not a BCP structure is a model of any of these theories? Since each of the axioms contains an existential part, the model relation can be demonstrated if one succeeds in finding a function $u$ to satisfy the corresponding axiom. Consider once more the BCP structure $\langle E, p\rangle$. It is a weak utility model, because axiom A.4 is satisfied with the function $u^{\prime}$ on $E$ : $u^{\prime}(e)=3, u^{\prime}(f)=2, u^{\prime}(g)=1$. It cannot be a strict utility model, because there does not exist a function $u$ to satisfy the necessary equalities. As to the strong utility theory, we were not able to find a function satisfying A.5, so we cannot take a decision on the model relation between $\langle E, p\rangle$ and the theories $T_{5}$. Fortunately, there exists another way to test these model relations.

Ever since the late fifties, a number of authors have been studying the equivalence relations between what we have labelled Type I theories and Type II theories (see for example Block \& Marschak, 1960; Luce \& Suppes, 1965). Simultaneously, the study of the formal foundations of measurement led to the formulation of sets of necessary and sufficient conditions for homomorphic mappings of particular algebraic relational systems to numerical relational systems (Suppes \& Zinnes, 1963; Krantz, Luce, Suppes \& Tversky, 1971). The study of probabilistic choice has also taken advantage of these results.

Some examples might illustrate these relations. If a BCP structure $\langle X, p\rangle$ is a weak utility model, then it is also a model of $T_{1}$, but not conversely. So realizing axiom A.I is a necessary, but not a sufficient condition for a BCP structure to be a model of the weak utility theory $T_{4}$. If $\langle X, p\rangle$ is a model of $T_{1}$, then it is also a model of $T_{4}$ if $X$ is finite. 
Consider a second example. If a BCP structure $\langle X, p\rangle$ satisfies A.6, and, hence, is a model of theory $T_{6}$, then it will also satisfy A.3 and be a model of $T_{3}$, but not conversely. The converse relation holds if the set $X$ is finite, and if $0<p_{x y}<1$ is true for all pairs of alternatives in $X$. A third example: A BCP structure satisfying A.5 will also satisfy A.2, but no sufficient conditions are known for a BCP structure satisfying A.2 to be a model of $T_{5}$.

These relations can help us a great deal in evaluating the model relation between a BCP structure and a Type II theory. If the BCP structure fails to satisfy a necessary condition, the model relation has to be rejected; if it satisfies a set of sufficient conditions, it is a model of the corresponding theory. In other cases, no decision can be made. Consider once more the BCP structure $\langle E, p\rangle$. It failed to satisfy A.3, so it cannot be a strict utility model. However, it satisfies A.2, so it still may be a model of the Fechnerian theory $T_{5}$.

According to some authors, only Type II theories should be called probabilistic choice theories. In their view, the axioms of Type I theories are just "observable properties" of choice behavior (see Luce \& Suppes (1965) for an example). We think that this distinction is both unwarranted and incorrect. Type I and Type II theories differ primarily in the content of their axioms. Type I theories formulate a set of constraints on the choice probabilities, whereas Type II theories state the existence of one or more functions defined over the set of altematives. In a way, both classes of theories can be seen as defining "properties" of probabilistic choice behavior, but neither the first set of axioms nor the second is "observable". It will be obvious that the utility functions $u$ are unobservable, but the same holds for the binary choice probabilities. Since we never observe choice probabilities as such, there is no way that a BCP structure might be a possible realization of empirical data.

\subsubsection{Models of data}

In evaluating the relations between theory and data, we will use the notion of possible realizations of data, which is similar to the notion of possible realizations of theory, presented earlier. As far as we know, Suppes (1962) has been the first to make this distinction. Due to the background of probabilistic choice modelling, our conception of 'models of data' differs slightly from his.

A possible realization of data will be a set-theoretical structure of the appropriate type, designed to incorporate all the valid information about the experiment that can be used in tests of the adequacy of the theory. In our view, "data" refers to "recorded observations", which refers to everything obtained from empirical observations through a coding or classification procedure.

In this Theory of Data, Coombs (1964) mad a distinction between two meanings of the term data, which is commonly used to refer both to the recorded 
observations, and to that which is analyzed. He preferred to reserve the use of the term to the latter. This distinction parallels the difference we make between data and model of data. A scientist interested in choice behavior, who has decided to design a choice experiment has an embarrassing richness of (potential) observations to make a selection from. From this abundance, only a few observations are actually recorded. The researcher registers the choices that are made, when, and by whom, and nothing more, for example. What is to be coded will depend on the theoretical framework used. In this respect, we never collect "data" as such: "Behavior does not yield data by parthogenesis." (Coombs, 1964).

The model of the data is nothing more than an orderly structure that capture the essence of these data; we call it a model of the data if it is a possible realization of the data. If, in our study of the probabilistic choice theories, a choice experiment is carried out, an ordered couple $\langle X, C\rangle$ can be a realization of the data, if it is defined as follows:

BC.1 $X$ is a nonempty set,

BC.2 $C$ is a set of binary-valued functions $c_{l}$, defined on $X \times X$, such that the following is true for every function and every pair of altematives:

$$
\left(c_{\mathrm{lyy}}=1 \wedge c_{l y x}=0\right) \vee\left(c_{l y \mathrm{y}}=0 \wedge c_{l y \mathrm{x}}=1\right) \vee\left(c_{\mathrm{Lyy}}=0 \wedge c_{l y \mathrm{z}}=0\right) .
$$

We call $\langle X, C\rangle$ a binary choice (BC) structure, reading $c_{l x y}=1$ as "the altemative has been chosen out of the option set $\{x, y\}$ by subject $l$ " and $c_{l y y}=0$ as "the alternative $x$ has not been chosen out of the option set $\{x, y\}$ by subject $l$ ".

A BC structure will be called a model of the data if the information on the data it contains is valid, both within the sense of the empirical observations and the fundamental probabilistic assumption. A complete evaluation of this validity relation is not easily made, since it involves checking a plenitude of assumptions, related to the data collection procedure used, the experimental design, and what Suppes (1962) has called "ceteris paribus conditions": disturbing environmental conditions, such as unwanted noises, bad lighting, and so on. To mention one, our basic probabilistic assumptions imply that every presentation of an option set of two altematives can be treated as equivalent to any other presentation of this option set. This assumption will not be automatically met and will require a careful design of the choice environment.

A model of the data is in general not unique. In addition to the BC structure mentioned earlier, a binary choice frequency (BCF) structure $\langle X, k\rangle$ can also be a possible realization of the same data, with $k$ defined as

$$
k_{x y}=\sum_{1} c_{i y x} .
$$


The BC structure is a model of the data if every comparison of the same two alternatives can be treated as similar, no matter when and where it is made; the BCF structure is a model of the data if these comparisons can be treated as similar, without taking notice of who makes them.

We do not gather data as we might collect autumn leaves; theoretical assumptions are generally not confronted with data in an unstructured way. Models of theory are well-organized structures in which all of a theory's assumptions are satisfied; one might alternatively look at theories as definitions of the classes of their models. On the other hand, models of data are close to Coombs' (1964) restricted definition of data: well-organized structures that capture the quintessence of recorded observations. Theory guides the selection of observations that are to be recorded, and theory guides the organization of these data into models.

\subsubsection{Theory versus data: evaluating goodness of fit}

Suppose we have a model of the data and we are interested in the relation between the observations and a particular probabilistic choice theory $T$. This means that we are interested in the question whether our theoretical assumptions can be maintained in the light of the empirical observations made. This does not imply that we will try to evaluate whether or not the model of the data (the binary choice structure) is a model of the theory. We cannot check axioms expressed in terms of choice probabilities in a BC structure.

By calculating the relative choice proportions we could obtain a structure that satisfies the definition of a BCP structure. Yet if such a structure of choice proportions failed to satisfy the relevant axioms, this would still be an inconclusive result. It will be hard to say whether this violation is a consequence of the latent BCP structure violating the axioms, or a corollary of binomial variability.

For Type II theories the following strategy is usually adopted (see Figure 3.1). One tries to construct a model of the theory that corresponds optimally (in some sense) to the model of the data. Most frequently, the likelihood will be the correspondence criterion. The likelihood function expresses the joint probability density of a BC structure, as a function of the estimated BCP structure. Because of the basic probabilistic assumption, each choice (i.e. each $c_{L y}$ ) is considered to be a realization of an independent Bemoulli trial, govemed by the choice probability $p_{x y}$. For example, to construct a strict utility model, the utility function $u$ is sought for which the likelihood is maximized, where the choice probabilities are calculated through the functional relation A.6. To a model of the Fechnerian theory $T_{5}$, a particular distribution function $H$ is selected and the maximum likelihood utilities $u$ are sought. 

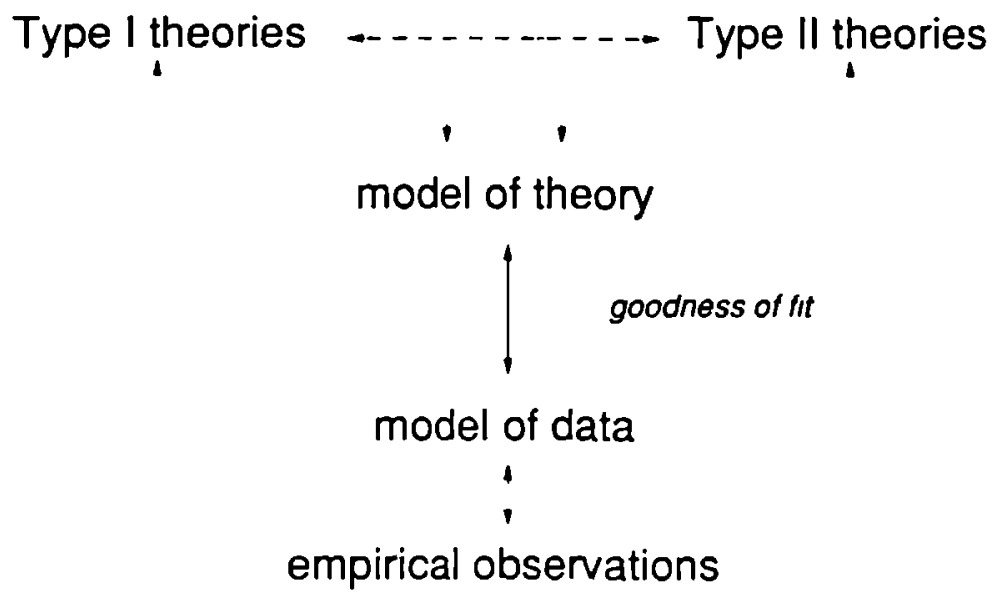

Figure 3.1 - Relations between theory, data, and models.

Suppose all this has been done. At this stage, a number of authors proclaim that they will continue with a "test of the model". This terminology cannot be correct. Obviously, the BCP structure obtained through the maximum likelihood strategy will be a model of the theory, by construction. On the other hand, the BC structure has to be a model of the data, for, if not, the whole procedure is meaningless. What is actually done at this stage is an evaluation of the correspondence relation between the model of the theory and the model of the data, a more common word for "correspondence" in this context being "goodness-of-fit".

If the maximum likelihood principle has been used, the likelihood function is the indicated device for this purpose. Within the Neyman-Pearson approach to statistical testing, the generalized likelihood ratio test statistic can be used to test the null hypothesis that the model of the data is a set of outcomes from a BCP structure that is a model of the theory, versus the altemative hypothesis that it is not.

It is worth reemphasizing that this is neither a test of the model relation between a theory and a BCP structure, nor a test of the model relation between the observations and a $\mathrm{BC}$ structure, but an evaluation of the statistical correspondence relation between both models in terms of the likelihood.

Unfortunately this likelihood ratio test is not very useful when it comes to evaluating why a model of the theory did not correspond to the model of the data. A rejection of the null hypothesis in a likelihood ratio test tends to be not 
particularly instructive: It tells us that the goodness of fit is in fact rather bad, without indicating why. However, there exists an alternative way of evaluating the relation between empirical observations and Type II theories. Suppose we were able to construct a model of a Type I theory, fulfilling the sufficient conditions for being also a model of a Type $\Pi$ theory, and showing an acceptable goodness-of-fit with the model of the data. In that case we know, without having estimated the utility function, that the BCP structure constructed will also be a model of the Type $\Pi$ theory. If, on the other hand, the model of the data shows a doubtful goodness-of-fit relation with the most plausible model of the Type I theory, and the axioms of this Type I theory are necessary conditions for the existence of a model of a Type $\Pi$ theory, it is similarly doubtful whether this Type II theory applies to the choices observed. Figure 3.1 represents these relations.

The axioms of Type II theories are useful for anyone looking for a latent structure in the data, to be interpreted as preferences goveming choices for example. The axioms of Type I theories have a more intuitive meaning, describing regularities in probabilistic choice behavior in terms of choice probabilities only, not in terms of some latent structure. Whereas Type II theories tend to have more practical applications, Type I theories offer better diagnostic tools in understanding and describing probabilistic choice behavior.

We do not claim that this idea of evaluating the goodness-of-fit of a model of the data with a Type II model through a model of a Type I theory is a new one. However, the evaluation of the correspondence relation between models of the data and models of Type I theories has usually been done in an unsatisfactory way. Luce and Suppes' quotation still applies to a major practice in the field:

"Lacking satisfactory statistical methods, authors often simply report, for example, the number of violations (...) and, on some intuitive basis, they conclude whether the failures are sufficiently numerous to reject the hypothesis. In other situations, tables or plots of data are reported and the reader is left pretty much on his own to reach a conclusion. Because the results are almost never clear cut, one is left with a distinct feeling of inconclusiveness."

(Luce \& Suppes, 1965, p.379)

Usually the evaluation between observations and theory is not based on the goodness-of-fit relation between a model of the data and a model of the theory, estimated on the basis of the maximum likelihood principle. Instead a structure of choice proportions is constructed and the model relation with the theory is evaluated. A failure of the relevant axiom does not usually lead to a rejection of the model relation, since, due to binomial variability, one is likely to find at 
least a number of violations. So the correspondence relation is only rejected with a sufficient number of violations. However, as Luce \& Suppes remarked, the determination of the acceptable number of violations is based on grounds that appear to be equally intuitive as shallow, and so is the assessment of the relation between theory and observations.

To give an example, take the following $\mathrm{BC}$ structure $\langle F, C\rangle$ which we take to be a model of the data, with $F=\{d, e, f, g, h\}$ and $C$ a set of 10 functions. Each pair of elements of $F$ has been presented 10 times. From this BC structure, a structure of binary choice proportions $\langle F, q\rangle$ can be obtained. Defining $q_{x y}$ as

$$
q_{x y}=\sum_{i=1}^{10} \frac{c_{l y y}}{c_{l y z}+c_{l y y}}
$$

we have

$$
\begin{aligned}
& q_{d \epsilon}=0.6, \quad q_{d f}=0.4, \quad q_{d g}=0.6, \quad q_{d h}=0.9, \quad q_{e f}=0.7 \text {, } \\
& q_{e g}=0.3, \quad q_{e h}=0.4, \quad q_{f g}=0.2, \quad q_{f h}=0.6, \quad q_{g h}=1.0 \text {. }
\end{aligned}
$$

One can easily check that the structure $\langle F, q\rangle$ of binary choice proportions fails to be a model of the strong stochastic transitivity theory $T_{2}$. It even fails to be a model of the weak stochastic transitivity theory, since there are four intransitive triples: $\{d, e, f\},\{d, f, g\},\{d, f, h\}$, and $\{e, f, h\}$. One may wonder whether or not these violations constitute enough evidence to reject the hypothesis that there exists a model of $T_{2}$ with an acceptable goodness-of-fit to $\langle F, C\rangle$. We have no instant answer to this question. All intransitive triples would have been transitive if $d$ had been chosen five times from the option set $\{d, f\}$ instead of just four times, and if $f$ had been chosen five times instead of six from $\{f, h\}$.

However, we can proceed in much the same way as we would do to evaluate the relation with a Type $\Pi$ theory. Taking the likelihood as the criterion, we can look for the "best fitting" model of $T_{2}$. Using a branch and bound algorithm developed for this purpose, we found that the BCP structure $\langle F, \hat{p}\rangle$ was the maximum likelihood strong stochastic transitivity model, with

$$
\begin{aligned}
& \hat{p}_{d e}=0.6, \quad \hat{p}_{d f}=0.5, \quad \hat{p}_{d g}=0.5, \quad \hat{p}_{d h}=0.9, \quad \hat{p}_{d f}=0.5 \text {, } \\
& \hat{p}_{\text {gg }}=0.25, \quad \hat{p}_{\text {eh }}=0.5, \quad \hat{p}_{f_{g}}=0.25, \quad \hat{p}_{/ h}=0.6, \quad \hat{p}_{g h}=1.0 \text {. }
\end{aligned}
$$

To obtain a test with an approximate size of .10 of the hypothesis that the BC structure was generated by a model of theory $T_{2}$, the generalized likelihood ratio test statistic was used. In this case, its value tumed out to be equal to 2099 . 
For an estimate of the value of the .9 quantile of the distribution of this statistic under the null hypothesis, we randomly generated 1000 choice structures satisfying strong stochastic transitivity. Using order statistics, our estimate of the .9 quantile tumed out to be .0847 . Therefore we do not reject the null hypothesis. Since $\langle F, \hat{p}\rangle$ is a model of $T_{2}$, a model of theory $T_{5}$ may exist for this structure of binary choices.

We can proceed in a similar way for other Type I theories. Contrary to evaluating the goodness-of-fit relation for each model of a Type II theory, the subsequent goodness-of-fit evaluation for models of hierarchically related Type I theories gives us more information on which theoretical assumptions on probabilistic choice survive a confrontation with the data, and which do not. It is our conviction that the construction of appropriate BCP models based on the maximum likelihood principle offers both a more promising way of evaluating the relation between theoretical assumptions and empirical observations, and an approach to the representation of choice probabilities which is more in line with the principles of axiomatic measurement theory.

\section{2}

\section{SAMPLING SCHEMES IN PAIRED COMPARISON TASKS}

In the previous section we described a strategy to evaluate the validity of probabilistic choice theories. The evaluation was based on an assessment of the correspondence between a model of the data and a model of the theory, and the likelihood was proposed as a suitable criterion. However, this layout of a strategy did not address a number of practical questions. One of the most important is: How do we arrive at a model of the data that allows us to make any statements on the validity of a theory. In this section, we will tentatively examine some answers to these questions. Furthermore, we will reflect on the question whether or not the probabilistic choice theories introduced earlier can be used to describe probabilistic choice behavior of a different nature. In these applications the uncertainty is not uniquely related to an unexplained inconsistency in a single choosing subject, but it is (also) tied to a scheme of sampling subjects from a heterogeneous population.

\subsubsection{Data collection design based on specified subjects}

If several, well specified subjects have answered at most once to questions on which alternative they would pick from all pairs from a (limited) set of alternatives, a model of these data may not be very adequate in answering questions on the potential validity of a particular probabilistic unfolding theory. With choice frequencies being one or zero, it will be hard to evaluate the correspondence with models of the various theories. Especially conditions 
defined on binary choice probability structures (a single subject's choice probabilities) would be very hard toevaluate; a distinction between the different forms of probabilistic transitivity will be impossible to achieve, and will be reduced to an mere evaluation of (deterministic) intransitivity.

A possible solution to this problem consists of confronting the subject with a series of repeated presentations of all pairs of altematives. This method has the advantage of increasing the possibility of building a statistical test with an acceptable power. Some authors have argued that, since probabilistic choice theories pretend to describe properties of asymptotic behavior, each pair of alternatives should be presented for as many trials as are needed to have some empirical evidence that the behavior is indeed "asymptotic" (cf. Luce \& Suppes, 1965, p. 377).

Alas, confronting a single subject with repeated presentations of the same set of all pairs of alternatives suffers from a considerable number of drawbacks. Several of them are merely practical. To name one, as the number of possible pairs increases with $n$ for every addition of one more altemative to a set of $n$ elements, a series of repeated presentations of all $n(n-1) / 2$ pairs will be a very time-consuming procedure, though one may resort to incomplete designs (cf. Bock \& Jones, 1968; Box, Hunter \& Hunter, 1978). A more principal objection however is that a model of these data may even fail to correspond with a model of the most general probabilistic choice theory. The basic assumption of probabilistic choice theories is that each choice from the same pair of altematives can be considered as an independent Bernoulli trial, where each alternative has a well-determined, non-changing probability of being chosen. This assumption is very susceptible to violations, even in an experimental design where each pair is presented only once to every subject. A particular sequencing of the presentations may lead to a task-specific structuring of the preferences, which could have tumed out otherwise if the sequencing had been different. None of these theories tries to account for this kind of memory effects, yet they are bound to occur in practical choice tasks.

Several of these problems have been addressed earlier by authors with an interest in (and a concem for) the paradigm of paired comparisons (e.g. David, 1988). One of the solutions has been the construction of so-called spaced presentation orders, in which all alternatives have a left-right balance in their presentations, and in which subsequent appearances of an altemative are interleaved in an intricate way.

When presenting all pairs is likely to lead to a task-induced structuring of the problem and a violation of the basic probabilistic choice assumption, these risks are only aggravated when subjects are asked to repeat their choices. If recall of earlier answers is a problem to be worried about in single presentations, it will make a paradigm of repeated presentations a questionable venture. It seems to us that such a paradigm will only be feasible in a choice situation in which 
it seems likely that memory effects can be ruled out, such as tasks with psychophysical stimuli or altematives that are otherwise difficult to identify.

Other strategies have been developed to circumvent these design difficulties. In the first experiment on probabilistic unfolding, Coombs (1958) tried to solve these problems by asking his subjects to rank subsets of four altematives, instead of making paired comparisons. By assuming that these rankings where reflections of underlying comparisons within pairs, Coombs "decomposed" them as such. However, Luce and Suppes (1965, sec 6.1) have successfully questioned this procedure, which is based on a rather strong assumption that need not be true.

\subsubsection{Data collection designs based on sampled subjects}

When Coombs (1958) introduced the first probabilistic unfolding theory, he did so with the intention of creating a theory to describe - or, altematively, explain - intrasubjective variability in choices. This variability was held responsible for the inconsistencies in choices that lead to the frequent failure of applications of the original, non-probabilistic unfolding methods. In this respect, probabilistic choice theories are the road of choice towards the preservation of the attractiveness of the unfolding paradigm: a merging of intersubjective unanimity in the perception of alternatives with individual differences in choices, leading to a powerful data-analytic tool.

All subsequent proposals of alternative probabilistic choice theories have kept the same spirit. In a fairly early stage, however, probabilistic choice theories were also used for an alternative purpose: the description of intersubjective variability in choices, as a consequence of the uncertainty related to a particular subject sampling scheme. Basically, this can be nothing but a remarkable mutation of the intentions of the original unfolding theory: individual subjects are not treated as such, but as manifestations of some 'generic' subject, as a member of a particular population of interest. An analysis of these choices does not try to describe individual differences, but treats them as deviations from some 'center of gravity' in the population.

One particular purpose this approach can be adopted for is a description of intersubjective dissimilarities between alternatives in choice tasks, rather than a description of individual differences in choices. In this kind of applications of probabilistic unfolding, the basic probabilistic choice assumption receives a new interpretation: We now assume that the process that can be described as an independent Bemoulli trial, with a constant probability of success, is whether or not a subject randomly selected from the population of interest chooses one alternative over another.

In the remainder of this section we will discuss the potential applicability of 
probabilistic choice theories for this type of observations, distinguishing between three out of four combinations of the following two assumptions: The individual ideals do (do not) coincide, the individual choices can (cannot) be described as random variables. The case of identical ideals and consistent individual choices bares no relevance to our topic. Throughout this exploration we will assume some form of intersubjectivity in the location of alternatives in the psychological space.

\section{(1) Different ideals - individual choices not random}

Assume we have a choice task in which several subjects choose once from every pair of alternatives, and made diverging choices. Assume we are willing to adopt the supposition that individual choices are not random, yet satisfy a latent unfolding structure: Each subject choose the alternative that most closely resembled his/her ideal. In that case, all intersubjective variability in choices has to be attributed to differences in individual ideals.

This is the kind of choice siruation Greenberg (1965) conceived his model specification for. In this case, individual choices need not be described probabilistically, yet the location of each ideal in the joint unfolding space can be described probabilistically. Greenberg's model specification is functionally identical to a non-parametric midpoint unfolding model. Bechtel's (1968) theory is based on a parametric specification of Greenberg's assumption. Bechtel suggested a normal distribution of the coordinates of ideals over the psychological continuum.

The midpoint unfolding theory, or the equivalent Zinnes-Griggs theory with the assumption of degenerate distributions of the altematives' coordinates, seems particularly apt for this kind of data. On the other hand, it is difficult to imagine how the existing random distance theories might be suited for these choice tasks, especially since they assume independent distance distributions. As to the strong unfolding models, we doubt whether they will prove to be suited.

\section{Same ideal - individual choices random variables}

If we assume that the ideals of all subjects coincide, but their individual choices have to be described as random variables, we arrive at a design that bares some resemblance with one of a single subject asked to repeat his choices. These assumptions may hold in tasks in which the choice criterion is comparatively unambiguous, leaving no room for intersubjective variability in its interpretation. A choice task with judgments of preference will rarely be appropriate for such assumptions, yet some tasks involving similarity judgments may be more suited. A task in which subjects are asked to choose from a pair of stimuli the one most similar to a reference stimulus can be easily 
viewed as one suited for an unfolding representation. In such a task, the ideal altemative is explicitly presented, not assumed to be implicitly present, as in preference tasks.

A distinction should be made between two extreme sampling schemes. In one, every subject makes only one choice at the most. In the other, more common sampling scheme, every subjects chooses from all possible pairs of feasible alternatives. This means that replications over different pairs of altematives are obtained within subjects, and replications within pairs are obtained across subjects. Takane (1987) has called this a multiple-judgment sampling scheme, and argues that probabilistic choice theories should take into account the systematic individual differences that give rise to statistical dependencies among observations.

Despite the apparent popularity of the multiple-judgment sampling scheme, this question of systematic individual differences has largely been overlooked in all probabilistic choice theories. Perhaps it is mathematical tractability that convinced researchers to stick with the questionable independence assumption. Others have even argued that doubts on the tenability of this statistical independence assumption are unwarranted (Indow, 1975).

If one neglects the validity of the independence assumption, the ZinnesGriggs theory may be adequate for these observations, if it seems plausible that distributions of the coordinates of ideal and altematives are identical for all subjects involved. All of the cases of the Zinnes-Griggs theory we distinguished earlier could apply: single sampling of the ideal or not, degenerate distributions of the coordinates of either altematives or ideal. For the Ramsay-Croon theory, a sufficient condition is the existence of identical distributions over all subjects for each perceived dissimilarity. For the random response theories, sufficient conditions are more difficult to establish.

\section{Different ideals - individual choices random variables}

An altemative set of suppositions is that ideals of subjects do not necessarily coincide, and that individual choices can be described as random variables. This is the combination of assumptions that we are most likely to encounter in choice tasks. Though subjects may agree in their behavior on the perceived similarity between altematives, they need not share opinions on the altemative that best suits the choice criterion.

The Zinnes-Griggs theory may be adequate for these observations, if it is plausible to assume identical distributions over subjects for the coordinates of all altematives, and if coordinates of the ideals can be described in terms of the population ideal and an additive, nomally distributed subject-related deviation. Obviously, the assumption of single sampling of the ideal coordinate cannot hold, so the only two cases left are those in which single sampling of the ideal 
is assumed, which implies correlated distributions of the distances.

It is very unlikely that the assumptions of the Ramsay-Croon random distance theories are fulfilled in this variety of choice situations. Both the theory proposed by Ramsay and its distributional variation presented by Croon assume independent distributions for both distances involved in arriving at a choice. Furthermore, Ramsay's and Croon's theories expect condition IP.3 to hold: The ideal alternative is always chosen. With subjects having divergent ideals, it is highly unlikely that they will always pick the altemative that is the "centroid", no matter from what option set. We have similar doubts on the applicability of the equivalent strong unfolding models, or, for that matter, all strong unfolding models.

Since a midpoint unfolding model is equivalent with a model of a variation of Zinnes-Griggs theory (single sampling of the ideal coordinate and degenerate distributions of the coordinates of the altematives), it is possible that a midpoint unfolding theory tums out to be adequate, if the description of the distribution of the ideal's coordinate over and within subjects is appropriate.

\subsubsection{Conclusion}

To evaluate the goodness-of-fit relation between a model that satisfies a particular property - strong stochastic transitivity, for example - and a model of data, one actually needs a model of data reflecting repetitive choices. Unfortunately, repeated choices sampling schemes with non-psychophysical stimuli are difficult to design.

On the other hand, probabilistic choice theories have been successfully used in choice tasks in which basically identical option sets are presented to several subjects and each subject is asked to choose only once from each option set. The model of the data is then built at the group or population level. For this reason, we will be using choice tasks with a multiple-judgment sampling scheme in our experimental evaluation of probabilistic unfolding theories.

We will try to build tasks in which all ideals coincide, and tasks in which the ideals differ. Similarity judgements may well be suited for the former situation. Asking subjects which one of two stimuli most closely resembles a third is a comparison that is functionally similar to a comparison of the similarity of two alternatives to an ideal one. Furthermore, explicitly presenting the "ideal" alternative has the additional advantage of allowing a test of the ideal point conditions. In order to make a valid test of these conditions, one needs to know the ideal a priori.

Testing properties of probabilistic choice behavior that are very much prone to violations in multiple-judgment tasks - as condition IP.3 is - can be questioned. We have indeed a priori doubts on the applicability of random distance 
theories that assume IP.3 to hold in tasks with this kind of sampling scheme.

Finally, we are willing to sidestep the questionability of the independence assumption in multiple-judgment tasks (different ideals). Though this is becoming a point of debate in the literature, existing probabilistic unfolding theories provide no means of incorporating these structural relations in their conceptual framework. While waiting for more appropriate theories, we will pretend that choices from distinct pairs by identifiable subjects are statistically independent. 



\section{$4 \quad$ Evaluating properties of probabilistic choice behavior}

The customary approach to assess the applicability of a probabilistic choice theory is based on an appraisal of an index of the goodness-of-fit between a model of the theory and a model of data, such as the likelihood of the data as a function of the model. To compare (models of) two theories, one can tum to a statistical test - a likelihood ratio test, for example - if the model of one theory can be considered as a submodel of the model of a second theory. The latter applies if the first model's parameters are a subset of the second's. A second approach in comparing models is based on the goodness-of-fit index taking into account the number of parameters needed to achieve it. In probabilistic choice modeling, this comes down to a penalized likelihood function, as in Akaike's information criterion AIC (Akaike, 1973, 1974).

These approaches are based on a more-or-less pragmatic conception of probabilistic choice theories and their representation techniques as "black boxes", exemplified in the global goodness-of-fit comparisons. Chapter 3 contained a proposal for an alternative strategy. Compared to the conventional approach, the advocated examination of necessary properties of probabilistic choice structures may lead to a more fine-grained appraisal of the relation between observations on choices and theories on probabilistic choice. This altemative approach can be more fair, independent as it is from differences in estimation techniques between various model specifications, and more instructive, since it is able to pin-point critical differences in the validity of assumptions on probabilistic choice each theory is willing to make. Furthermore, the same statistical machinery that is used for making global goodness-of-fit assessments can be used for making evaluations on properties.

Our aim then is to evaluate the goodness-of-fit relation between models incorporating these assumptions and models of data. We want to assess the validity of a probabilistic unfolding theory through properties of systems of binary choice probabilities, whenever these properties act as necessary conditions for the existence of a model of a particular theory.

This chapter contains a description of an evaluation scheme developed for this purpose. This scheme is based on maximum likelihood estimation of binomial probabilities under conditional partial order restrictions, a branch search strategy, and a generalized likelihood ratio test. 
With one exception, the properties described in Chapter 3 were expressed in terms of conditional inequalities on choice probabilities. These inequalities can be regarded as conditional ordinal restrictions that have to be taken into account in obtaining estimates of choice probabilities for a model of data. Section 4.2 contains a summary of these ordinal restrictions, and an analysis of their conditional nature. In general, the respective conditions can be expressed in terms of a ranking of the feasible altematives.

If this ranking is known a priori, obtaining maximum likelihood estimates is not difficult, since the characteristics of constrained maximum likelihood estimates of partially ordered binomial parameters are well known. Estimates can be found through the isotonic regression of the choice proportions, with the number of presentations as weights. Several algorithms to calculate the solution to the isotonic regression have been proposed in the literature. Section 4.3 contains a description of a new, efficient algorithm. This algorithm is general enough to cover all but one of the partial orders, and sufficiently specific to allow efficient implementations. One additional algorithm, for moderate stochastic rankings, is also presented.

If the ranking for a specific property is unknown (the more common case) the restrained maximum of the likelihood function has to be found within the set of all feasible rankings. Estimates that accompany the ranking with the highest likelihood are maximum likelihood estimates. In section 4.4 , a branch search strategy is offered for this purpose .

The assertion that a property holds can be reformulated as an statement on the location of the vector of parameters in a well-defined subset of the parameter space, i.e. a statistical hypothesis. To evaluate the applicability of a condition, the generalized likelihood ratio test principle will be used. Section 4.5 describes this test and discusses the behavior of the test statistic under the null hypothesis.

The sixth section contains some examples of this evaluation strategy, applied to models of data published in the literature.

\subsection{PRELIMINARIES}

As in the earlier sections, we assume we are dealing with a nonempty set $X$ of $\kappa_{x}$ feasible decision altematives. The general model of data is a system of binary choice frequencies $\langle X, K\rangle$, with $K$ a set of choice frequency functions $k$ on $X \times X$. To ease the exposition, $K$ will be considered to be a singleton unless explicitly stated otherwise; $k_{x}$ assigns the number of presentations of $\{x, y\}$ after which $x$ has been chosen.

Let $n$ and $q$ be functions on $X \times X$, such that 


$$
\begin{aligned}
& n_{x y}=k_{x y}+k_{y x} \\
& q_{x y}=\frac{k_{x y}}{k_{x y}+k_{y x}} .
\end{aligned}
$$

In words, $n_{x y}$ is the number of presentations of the couple $\{x, y\}$, and $q_{x y}$ is the choice proportion of $x$ in this couple. Because of the basic probabilistic choice assumption, choice frequencies $k_{x y}$ are considered to be values from independent binomial distributions with parameters $\left(n_{x y}, p_{x y}\right)$.

The general model of a probabilistic choice theory is a binary choice probability (BCP) system $\langle X, P\rangle$ with $P$ a set of $\kappa_{P} B C P$ functions. Since we are dealing with forced pairwise comparisons, so that $p_{y x}=1-p_{x y}$ holds, and since couples $\{x, x\}$ are never presented, the parameter space $\theta$ for the BCP system $\langle X, P\rangle$ is a unit hypercube of dimensionality $\left(\kappa_{x}\left(\kappa_{x}-1\right) \kappa_{p}\right) / 2$.

The likelihood function $l_{x}(k ; g)$ is defined as the joint probability of the structure of binary choice frequencies $\langle X, k\rangle$, regarded as a function of an unknown BCP structure $\langle X, p\rangle$

$$
l_{X}(k ; p)=\prod_{X}\left(\begin{array}{l}
n_{x y} \\
k_{y y}
\end{array}\right) p_{x y}^{k_{y y}}\left(1-p_{x y}\right)^{\left(n_{y y}-k_{x y}\right)}
$$

where the product runs over all pairs $(x, y)$ in $X$. A real-valued function $g$ on $X \times X$ assigns maximum likelihood estimates of the binary choice probabilities if $l_{X}(k ; g) \geq l_{X}(k ; f)$ for any admissible function $f$.

We will use $r(X)$ to denote a ranking of the altematives in $X$, and $R(X)$ to denote the set of all possible rankings of $X$. If $x$ is preceded by $y$ in a ranking, the shorthand notation $\overrightarrow{x y}$ is used.

\subsection{ORDINAL RESTRICTIONS AND RANKINGS}

In our evaluation stratgey, the properties discussed in Chapter 2 will be used as conditional restrictions in obtaining estimates of the choice probabilities. Only one of these properties (IP.3) was expressed in terms of a conditional equality restriction; all other properties were formulated as conditional ordinal restrictions on choice probabilities. In general, the conditions generate partial orders on the binomial parameters. In this section, the conditionality of these ordinal restrictions will be reconsidered. Each condition will be expressed in terms of a particular ranking, or set of rankings, of the elements in $X$. 
Table 4.1

Restrictions corresponding to the three unidimensional unfolding properties.

\begin{tabular}{|c|c|c|c|c|c|}
\hline \multirow[t]{2}{*}{ Condition } & \multirow[t]{2}{*}{ Ranking } & \multicolumn{4}{|c|}{ Restrictions } \\
\hline & & $p_{w \pi} p_{m y}$ & $p_{m y} p_{x y}$ & $p_{w r} p_{n w}$ & $p_{x y} p_{y x}$ \\
\hline. $\mathrm{CM}$ & $\overrightarrow{w x y}$ & $p_{w x} \leq p_{w y}$ & $p_{m y} \leq p_{x y}$ & & \\
\hline $\mathrm{BM}$ & $\frac{\overrightarrow{w x y z}}{w x z y}$ & $p_{w x} \geq p_{w y}$ & $\begin{array}{l}p_{m y} \leq p_{x y} \\
p_{w y} \leq p_{x y}\end{array}$ & $\begin{array}{l}p_{w \pi} \leq p_{n w} \\
p_{w x} \leq p_{N w}\end{array}$ & $p_{x y} \leq p_{y z}$ \\
\hline $\mathrm{BCM}$ & $\begin{array}{l}\overrightarrow{w x y z} \\
\overrightarrow{w x z y}\end{array}$ & & $\begin{array}{l}p_{m y} \leq p_{x y} \\
p_{m y} \leq p_{x y}\end{array}$ & $\begin{array}{l}p_{w x} \leq p_{s w} \\
p_{w x} \leq p_{m w}\end{array}$ & $p_{x y} \leq p_{y x}$ \\
\hline
\end{tabular}

Note - CM: charactenstic monotonicity, BM: bilateral monotonicity, BCM: bilateral/characteristic monotonicity; the ideal alternative is denoted as $z$.

\subsubsection{Ideal point conditions}

The conditional nature of the ordinal restrictions in the ideal point conditions IP. 1, and IP. 2 is rather straightforward. The ordinal restrictions depend on which one of the altematives (if any in $X$ ) is the ideal for a BCP structure. These restrictions represent subsets $\Theta^{\mathbb{I P} 1}, \Theta^{\mathrm{IP} 2}$ of the parameter space. Similarly, the ordinal restrictions for the ideal point condition IP.4 represent a subset $\Theta^{\mathbb{P} 4}$.

The unilaterally decreasing/increasing property [2.13-14], the bilateral property [2.15] and the symmetric property [2.16] are each conditional upon the ideal alternatives for the respective BCP structures and the unfolded order. The unfolded order was defined as a ranking of the alternatives that is monotonic with respect to the ordering of the corresponding coordinates, which is called a qualitative $J$ scale in traditional unfolding terminology.

\subsubsection{Unidimensional unfolding}

The conditional nature of the properties in unidimensional unfolding characteristic monotonicity (CM), bilateral monotonicity (BM), and bilate$\mathrm{ral} /$ characteristic monotonicity (BCM) - is well defined. The inequalities are conditional on the unfolded order and, for bilateral and bilateral/characteristic monotonicity, on the relative location of the ideal in the underlying psychological space. These restrictions are summarized in Table 4.1. 

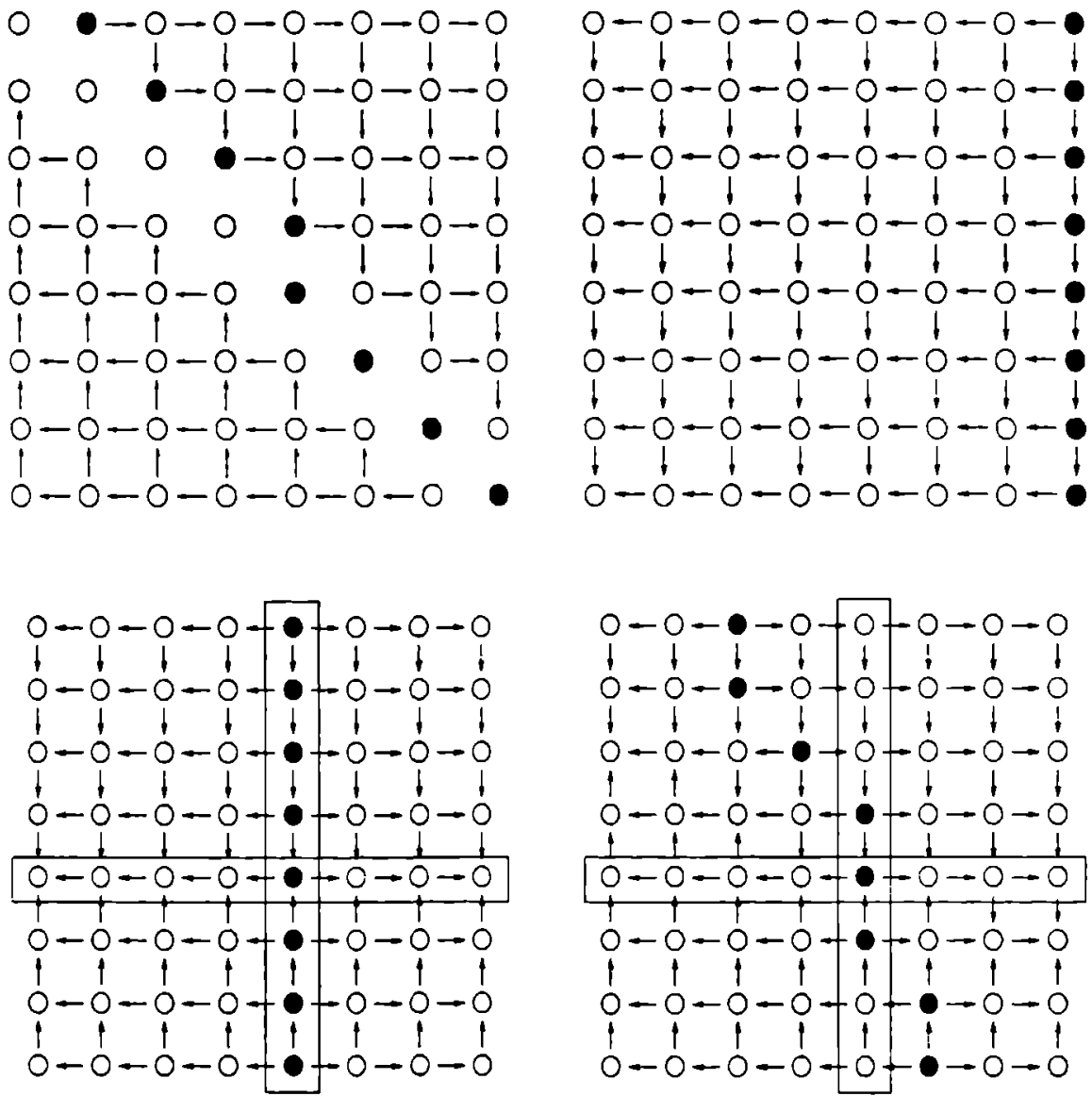

Figure 4.1 - Inequalities for CM (top left), SST (top right) and examples for BM (bottom left) and BCM (bottom right) in a matrix of chorce probabilities. Row and column indices are arranged in the unfolded order $(\mathrm{CM}, \mathrm{BM}, \mathrm{BCM})$ or a strong stochastic ranking (SST). Each cell contains the probability of choosing the row element. Arrows point to the higher probability. For $\mathrm{BM}$ and $\mathrm{BCM}$, the cells in the rectangle correspond to the element closest to the ideal. Row minima have a darker shade. Patterns of row minima for $\mathrm{CM}$ and $\mathrm{BCM}$ are but examples.

The ordinal restrictions of characteristic monotonicity are rather simple. These restrictions are conditional on the unfolded order only. If choice probabilities satisfying characteristic monotonicity are organized in a matrix with the row and column indices in the unfolded order, the choice probabilities $p_{x y}$ in a row do not increase from the left to the main diagonal, and do not decrease 
from the main diagonal to the right. The minima in each row are located either on the main diagonal, or in the cell immediately to the right or to the left of the main diagonal. Figure 4.1 (top left) contains an example of a pattem satisfying characteristic monotonicity. The arrows point to the higher probability, and the row minima have a darker shade.

The restrictions of bilateral monotonicity are conditional on the unfolded order and on the location of the ideal altemative in the unfolded order. If choice probabilities satisfying bilateral monotonicity are organized in a matrix with the row and column indices in the unfolded order, the choice probabilities $p_{\mathbf{r y}}$ in a row corresponding to the aitemative $x$ reach a minimum that does not depend on $x$, but on whether or not $y$ is identical to or closest to the ideal altemative. Figure 4.1 (bottom left) contains an example of a pattem satisfying bilateral monotonicity. The cells in the row and in the column that correspond to the element closest to the ideal are each enclosed in a rectangle.

The conditional inequalities that make up bilateral/characteristic monotonicity are more complex than the two properties just mentioned. Speaking once again in terms of a matrix of choice probabilities with the row and column indices in the unfolded order, the probabilities in each row reach a minimum that depends on the location of the row index in the unfolded order. If the row index corresponds to the ideal altemative, or the altemative that is closest to the ideal, the minimum in the corresponding row is reached in the column with the same index (i.e. on the main diagonal), and is necessarily equal to one half. Otherwise, the minimum can be located within a range of columns, bounded by the column with the same index as the row index and the column with the index closest to the ideal, the latter column included. Yet this set of row minima is further restricted by the property that for any row index more removed from the ideal but located at the same side of one particular row index, the row minimum cannot be located in a column with an index closer to the ideal.

Figure 4.1 (bottom right) contains an example of a pattern satisfying bilateral monotonicity. The cells in the row and in the column that correspond to the element closest to the ideal are each enclosed in a rectangle. The minimum in each row has a darker shade. One can see from this figure that the pattem of row minima is bounded by BM (all row minima in the rectangle) and CM (all row minima on or next to the main diagonal). If one connects the minima in the consecutive rows with a line, starting in the top row, the line should run from top to bottom, and from left to right, never making a left curve.

\subsubsection{Stochastic transitivity}

The premises in the expressions for stochastic acyclicity, and weak, moderate, strong and strict stochastic transitivity [2.1-2.5] were expressed in terms of 
binary choice probabilities, not in terms of a ranking. These sets restrictions can be related to well-specified classes of rankings. Consider the binary preference relation induced by the BCP function $p$ (cf. Chapter 2):

$$
y D x \Leftrightarrow p_{y x}<p_{x y} .
$$

Next to this preference relation, we can define a binary equivalence relation, similarly induced by $p$ :

$$
y E x \Leftrightarrow p_{y x}=p_{x y} .
$$

The relation $D$ in [4.3] is necessarily asymmetric, and the relation $E$ in [4.4] is necessarily symmetric. Other properties of these relations $D$ and $E$ depend on the level of stochastic transitivity satisfied in $\langle X, p\rangle$.

(a) The relation $D$ on $X$ is acyclic if and only if $\langle X, p\rangle$ satisfies stochastic acyclicity; in that case $D$ generates a suborder on $X$.

(b) the relation $D$ on $X$ satisfies negative transitivity - if $w D y$ then either $w D x$ or $x D y-$ if and only if $\langle X, p\rangle$ satisfies weak stochastic transitivity; in that case $D$ generates a weak order on $X$, and relation $E$ on $X$ satisfies transitivity.

Take a ranking rof the elements in $X$. Call such a ranking isotonic with respect to $D$ ( $D$-isotonic) if, whenever $y$ is preceded by $x$ in this ranking - denoted as $\overrightarrow{y x}$ - the pair $(x, y)$ is not in $D$. Such a ranking exists if and only if the relation $D$ is acyclic.

In the remainder the subproblem will be considered of finding maximum likelihood estimates for the various stochastic transitivity conditions, conditional on a ranking of the elements in $X$ known to be $D$-isotonic.

Call a ranking $r(X)$ of the elements in $X$ a weak stochastic (WS) ranking if the following holds for any two elements $y, x \in X$ :

$$
\overrightarrow{y x} \Rightarrow p_{y x} \leq p_{x y}
$$

Call it a moderate stochastic (MS) ranking if the following holds for any three elements $y, x, w \in X$ :

$$
\overrightarrow{y x w} \Rightarrow p_{y x} \leq p_{r y} \wedge p_{x w} \leq p_{w x} \wedge\left(p_{y w} \leq p_{x w} \vee p_{y w} \leq p_{y x}\right)
$$

and a strong stochastic (SS) ranking if the following holds 


$$
\overrightarrow{y x w} \Rightarrow p_{y x} \leq p_{x y} \wedge p_{x w} \leq p_{w x} \wedge p_{y w} \leq p_{x w} \wedge p_{y w} \leq p_{y x}
$$

A weak, moderate or strong stochastic ranking is in general not unique. In the extreme case where $p_{x y}=p_{y x}$ for all pairs $(x, y)$ in $X \times X$, all rankings satisfy $D$-isotonicity, and are weak, moderate, and strong stochastic rankings.

Clearly, a weak stochastic ranking exists if and only if $D$ is acyclic or, alternatively, if the BCP structure $\langle X, p\rangle$ satisfies stochastic acyclicity (STA), and vice versa. Let $\Theta_{r(X)}^{\text {STA }}$ be the subset of the parameter subspace that corresponds to stochastic acyclicity, conditional on the ranking $r(X)$ of the elements in $X$ that is known to be $D$-isotonic.

Since the inequalities of weak stochastic transitivity (WST) are a superset of those of STA, a weak stochastic ranking exists if a BCP structure satisfies WST. Yet the converse does not necessarily hold. The subset $\Theta_{r(X)}^{\text {wST }}$ corresponding to WST is but a subset of $\Theta_{r(x)}^{\text {STA }}$, obtained by removing some of the boundary planes of the $[.5,1]$ hypercube corresponding to STA. For this reason, a vector of estimates for which the likelihood is maximized, conditional on a ranking known to be $D$-isotonic, does not necessarily exist. If the conditional maximum under STA is located in the parameter subspace $\Theta_{r(x)}^{\text {WST }}$ that corresponds to WST, this is the conditional maximum under wST. If it is not, the conditional maximum under wST does not exist.

Take the set $A=\{a, b, c\}$, the ranking $r(A)$ with $\overrightarrow{c b a}$ known to be $D$-isotonic, and suppose we have

$$
k_{a b}=8 \quad k_{a c}=4 \quad k_{b c}=9 \quad n_{a b}=n_{a c}=n_{b c}=10 .
$$

The following function $f$ on $A \times A$ assigns maximum likelihood estimates of the choice probabilities, under the condition that the ranking $r(A)$ with $\overrightarrow{c b a}$ is a weak stochastic one:

$$
f_{a b}=.8 \quad f_{c c}=.5 \quad f_{b c}=.9 .
$$

Clearly, this is not the conditional maximum under WST, which would require $f_{a c}>$.5. In fact, this set of values [4.8] results in the lowest upper bound of the likelihood function under WST. This holds in general: Restricted maximum likelihood estimates conditional on a ranking known to be weak stochastic result in the lowest upper bound of the likelihood function under weak stochastic transitivity.

A similar problem holds for moderate stochastic transitivity (MST). If the BCP structure $\langle X, p\rangle$ satisfies MST, a moderate stochastic ranking exists, but the reverse does not generally hold. Take the following BCP structure $\langle A, p\rangle$, with $A=\{a, b, c\}$ and 

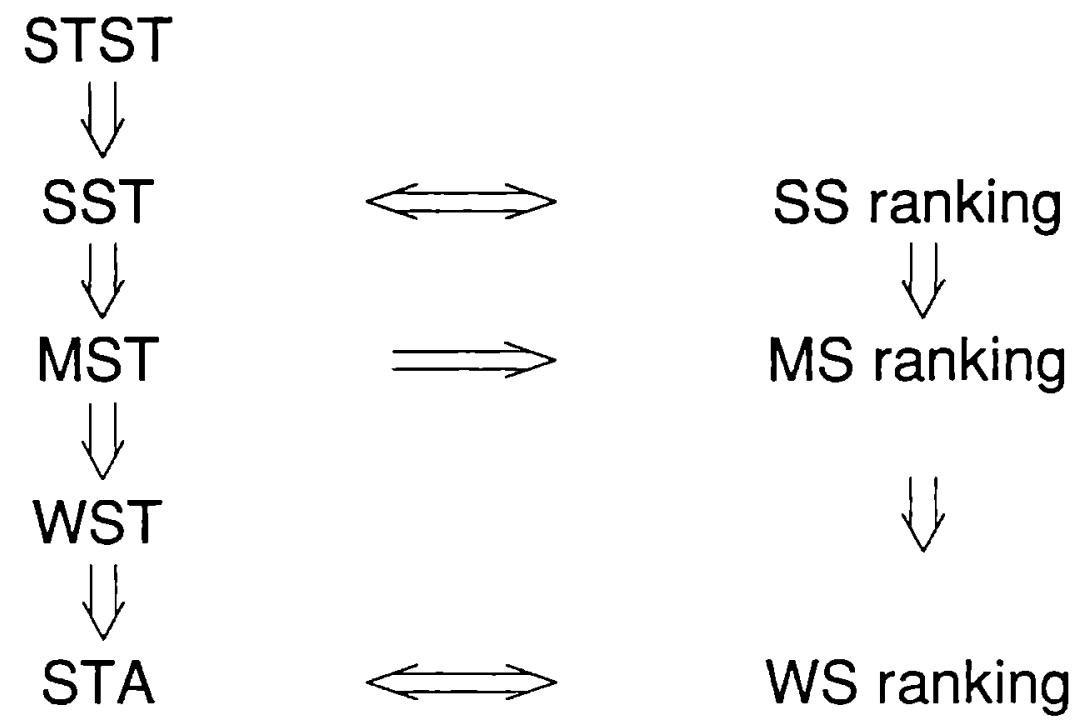

Figure 4.2 - Relations between the stochastic transitivity conditions and classes of $D$-isotonic rankings.

$$
p_{a b}=.8 \quad p_{a c}=.5 \quad p_{b c}=.5 .
$$

The ranking $\overrightarrow{c b a}$ would be an MS ranking, but this structure fails to satisfy WST. To check this, consider the premises $p_{b c}=.5, p_{c a}=.5$, and the failure of the conclusion $p_{b a}=.2$.

Assume the ranking $r(X)$ is known to be moderate stochastic, and let $\Theta_{\alpha X)}^{\mathrm{Ms}}$ be the subset of the parameter space in which the vector of estimates satisfies the corresponding restrictions [4.6]. The maximum of the likelihood function in this subset can always be found. The subset of the parameter space $\Theta_{x,(x)}^{\mathrm{MST}}$ that corresponds to moderate stochastic transitivity is equal to the intersection of $\Theta_{\{(x)}^{\mathrm{MS}}$ and $\Theta_{\{(x)}^{\text {WST }}$. If the vector of maximum likelihood estimates conditional on a moderate stochastic ranking satisfies wST, the maximum under MST has been found. In general, the estimates for a moderate stochastic ranking result in the lowest upper bound of the likelihood function under MST.

If the BCP structure $\langle X, p\rangle$ satisfies strong stochastic transitivity (SST), a strong stochastic ranking of the elements in $X$ exists. The reverse also holds. The subset $\Theta_{\alpha(X)}^{\text {wST }}$ contains $\Theta_{\gamma(X)}^{s s}=\Theta_{,(X)}^{\text {sST }}$ as a proper subset. If choice probabilities satisfying SST are organized in a matrix with the row and column indices corresponding to the strong stochastic ranking, the probabilities in each row do not decrease from the left to the right (Figure 4.1). 
If the BCP structure $\langle X, p\rangle$ satisfies strict stochastic transitivity (STST), a strong stochastic ranking of the elements in $X$ exists, but the reverse does not necessarily hold. The subset $\Theta_{f(X)}^{\text {STST }}$ of the parameter space that corresponds to STST is a subset of $\Theta_{\{(X)}^{S S T}$, but $\Theta_{\{(X)}^{S T S T}$ is closed nor convex. In general, the restricted estimates for a strong stochastic ranking result in the lowest upper bound under - STST.

All five levels of stochastic transitivity can be related to particular classes of rankings. Figure 4.2 illustrates these relations. The existence of a ranking of a particular class - say, a moderate stochastic one - is in general a necessary, but not a sufficient condition for the fulfillment of the corresponding stochastic transitivity condition (moderate stochastic transitivity, in the example). The lowest upper bound of the likelihood function, conditional on such a transitivity condition, can always be determined; the conditional maximum of the likelihood cannot always be found for the properties weak, moderate and strict stochastic transitivity.

\subsection{MAXIMUM LIKELIHOOD ESTIMATES OF BINOMIAL PROBABILITIES UNDER ORDINAL RESTRICTIONS}

This section discusses the problem of obtaining maximum likelihood estimates under ordinal restrictions.

\subsubsection{Basic principles}

Let $Y$ be a subset of ordered couples from $X$, in such a way that $Y$ is an asymmetric binary relation on $X$. In addition, define the asymmetric binary relation $Y^{C}$ on $X$ through

$$
(x, y) \in Y \Leftrightarrow(y, x) \in Y^{C}
$$

Read $l_{Y}(k ; g)$ as in [4.2], with the product restricted to elements of $Y$.

Let $R$ be a reflexive, transitive binary relation on $Y$. Such a relation $R$ establishes a partial order on the set $Y$. Later on, we will be dealing with relations $R$ on $Y \cup Y^{c}$, but making the restriction to $Y$ eases the discussion without hindering the generalizability of its principles.

Suppose estimates $g_{x y}$ of the BCP probabilities $p_{x y}$ for elements $(x, y),(v, w) \in Y$ are known to satisfy the following restrictions:

$$
(x, y) R(v, w) \Rightarrow g_{x y} \leq g_{v w} .
$$


Given a structure of choice frequencies $\langle X, k\rangle$, the problem of finding maximum likelihood estimates of the probabilities $p_{x y}$ in $Y$ conditional on $R$ consists of finding, within the set of all functions $g$ on $Y$ that satisfy [4.11], the function $f$ for which $l_{Y}(k ; f)$ is maximized.

It is well known that when $R$ is empty, the choice proportions are maximum likelihood estimators. Therefore, if the restrictions [4.11] are satisfied by the function $q$, maximum likelihood estimates of the $p_{x y}$ are equal to the respective choice proportions $q_{x y}$. If $q$ fails to satisfy the restrictions, some other function has to be found.

The special case of relations $R$ that generate a linear order has been described first by Ayer, Brunk, Ewing, Reid and Silverman (1955). The more general partial order has been discused by Van Eeden (1956, 1957a, 1957b). The function we are looking for can be characterized in terms of the isotonic regression: Maximum likelihood estimates of the $p_{x y}$ under partial order restrictions are provided by the isotonic regression $q^{*}$ of the choice proportions $q$ with weights $n$ on the partial order (the reader may consult Robertson, Wright \& Dykstra (1988) for a proof).

The calculation of the isotonic regression can be regarded as a quadratic programming problem, on which a vast literature exists. Due to its special nature, a series of more specific computation algorithms have been proposed. Most of these algorithms have been reviewed by Barlow, Bartholomew, Bremner and Brunk (1972, Chapter 2.3), whose review has been updated by Robertson, Wright and Dykstra (1988, Chapter 1.4).

In general, the isotonic regression $q^{*}$ induces a partition of $Y$ into subsets in which $q^{*}$ is constant. These subsets are usually called solution blocks, or level sets, and the value of $q^{*}$ in such a solution block is equal to the weighted average of the choice proportions corresponding to the elements in this block. Computation algorithms differ in their strategy to assign elements to solution blocks. Most algorithms deal with a particular class of relations $R$ only. For example, Kruskal's (1964) "Up-and-down B locks" algorithm - which has been developed for multidimensional scaling, and is rather easy to implement - can be used if $R$ generates a simple order on $Y$.

Unfortunately, the relations that follow from the properties discussed in section 4.2 do not generate simple orders of the choice probabilities, but less well-structured partial orders. As Robertson, Wright and Dykstra (1988) observed, algorithms to compute the isotonic regression for partial orders "(...) usually require complicated checking and branching logic and can be difficult to understand and to program". 
Dissatisfied with the speed, the efficiency and the difficulties in implementing one of the existing algorithms, we developed a new, general algorithm, tailored to the restrictions on binomial probabilities we are dealing with, yet sufficiently general to cover all properties. The remainder of this section contains a description of the principles of this algorithm, which itself is described in the following section (4.3.2).

To start, define two functions $n$ and $q$ on the power set of $Y$, such that $n_{\varnothing}=0$ and $q_{\emptyset}=0$, and for each nonempty subset $B \subseteq Y$,

$$
\begin{aligned}
& n_{B}=\sum_{(x, y) \in B} n_{x y} \\
& q_{B}=\frac{\sum_{(x, y) \in B} n_{x y} q_{x y}}{n_{B}} .
\end{aligned}
$$

In words, $n_{B}$ contains the sum of all presentations and $q_{B}$ the weighted average of the choice proportions in $B$, where $B \subseteq Y$ is a subset of the set of ordered couples from $X$.

The basic principles of the algorithm are embodied in Lemma 4.1 and Lemma 4.2.

\section{LEMMA 4.1}

If, for the elements of an asymmetric binary relation $B$ on $X, B \subseteq Y$, all estimates are equal to a constant, then the function $l_{B}$ is maximized for $f_{x y}=q_{B}$, for all $(x, y)$ in $B$.

\section{PROOF}

Let $f_{x y}=s$ for all elements of $B$. Obviously, this function satisfies the restrictions [4.11]. We can express $l_{B}$ as a function of $s$ :

$$
\begin{aligned}
l_{B}(k ; s) & =\sum_{(\alpha, y) \in B} k_{x y} \ln s+k_{y x} \ln (1-s) \\
& =\left[q_{B} \ln s+\left(1-q_{B}\right) \ln (1-s)\right] n_{B} .
\end{aligned}
$$

The likelihood $l_{B}(k ; s)$ as a function of $s$ reaches a unique maximum at $s=q_{B}$. QED

Some additional terminology is needed. A partition of a subset $B \subseteq Y$ into $k(1<k)$ disjoint subsets $B$, will be called an $R$-consistent partition if for every two subsets $B_{1}, B_{\text {, with }} q_{B_{1}}<q_{B}$, there are no elements $(v, w) \in B_{i}$ and $(x, y) \in B_{\text {, }}$ 
for which $(x, y) R(v, w)$ holds. Call such a partition of $B$ into subsets $B$, the maximal $R$-consistent partition if there does not exist an $R$-consistent partition of any of the subsets $B_{1}$.

\section{LEMMA 4.2}

Let $g_{x y}=q_{B}$ for all $(x, y) \in B \subseteq Y$. The following two statements are equivalent.

(1) There exists an $R$-consistent partition of $B$.

(2) There exists a function fon B that satisfies the restrictions [4.11] and increases the likelihood of the frequencies in $B$ : $l_{B}(k ; f)>l_{B}(k ; g)$.

\section{PROOF}

First we will show that (2) follows from (1). Because of

$$
q_{B}=\frac{\sum_{i=1}^{k} n_{B_{i}} q_{B_{i}}}{n_{B}}
$$

and the convexity of $l_{B}(k ; s)$ as a function of $s$, the result follows. To show that (1) follows from (2), create a partition of $B$ by assigning any two elements $(x, y),(v, w)$ to the same subset $B$, if and only if $f_{x y}=f_{v w}$. This partition will contain at least two elements, for otherwise $f=g$ and $l_{B}(k ; f)=l_{B}(k ; g)$. QED

The following theorem now can be proven.

THEOREM 4.1

If, for a subset $B \subseteq Y$, either

(1) there exists an $R$-consistent partition of $B$, this partition is the maximal $R$-consistent partition of $B$, and for all $(x, y)$ in each subset $B_{i}$ in this partition $f_{x y}=q_{B_{i}}$ or

(2) there does not exist an $R$-consistent partition of $B$, and $f_{x y}=q_{B}$, then the function $f$ maximizes the likelihood of the choice frequencies $k$ in $B$ conditional on $R$. 


\section{PROOF}

Suppose there exists a function $g$ on $B$ such that $l_{B}(k ; g)>l_{B}(k ; f)$. Through Lemma 4.2 , the latter implies that there exists an $R$-consistent partition of this subset $B$. Since this contradicts the assumptions, such a function $g$ cannot exist. Hence, the likelihood is maximized for the function $f$.

QED

\subsubsection{The algorithm}

Obviously, to find maximum likelihood estimates, one could start by taking the function $f_{x y}=q_{Y}$, and check whether an $R$-consistent partition of $Y$ exists. If there is one, recursively checking for the existence of $R$-consistent partitions in the resulting subsets $B_{1}$ would bring us to the maximal $R$-consistent partition. Though this algorithm will lead to the desired estimates, it is not a very economical one. Inspired by an algorithm for isotonic regression with one or more independent variables, proposed by Gebhardt (1970), we developed a more efficient algorithm. It is also based on Theorem 4.1 , but it handles the successive partitioning in a more prudent way.

To get an intuitive grasp of the way the algorithm functions, the reader may think of Kruskal's (1964) "Up-and-down Blocks" algorithm, to which our algorithm reduces if the relation $R$ generates a simple order on $Y \cup Y^{C}$. In the "Up-and-down Blocks" algorithm, the elements of $Y \cup Y^{C}$ are arranged in a ranking that is isotonic with respect to $R$ ( $R$-isotonic), i.e. arranged in accordance with the (simple) order restrictions. The algorithm then starts at one side of the ranking, and works its way through the ranked elements to the other side, pooling elements in solution blocks if restrictions are violated. If elements are pooled, the algorithm has to check whether the partition it has left behind is still $R$-consistent.

If the relation $R$ generates only a partial order on $Y \cup Y^{C}$, things are more complicated. In general, not one but several rankings are $R$-isotonic, and if we applied the "Up-and-down Blocks" algorithm to all of these rankings, it is very well possible that we would end with different partitions. As a corollary, the isotonic regressions on these linear orders need not all be equivalent in terms of likelihood.

The non-uniqueness of the $R$-isotonic ranking is the main bother. If we have a relation $R$ generating a partial order on a set, and a ranking that is consistent with this relation $R$, the partition into solution blocks generated by the "Upand-down Blocks" algorithm is not necessarily maximal, i.e. there is no guarantee that the resulting solution blocks cannot be further partitioned. We therefore need additional logic to examine each element of the partition for the existence of a further $R$-consistent partition. This is taken care of in step (4) of 
the algorithm to be discussed below.

The algorithm proceeds in two main steps, each divided in cycles. First we consider the subset of $R$ that is totally contained in $Y$. Then we will consider the remaining elements of $R$. Speaking in terms of a matrix of choice probabilities, as for the properties represented in Figure 4.2, we will first deal with the restrictions in the lower (or upper) diagonal part of the matrix (first step), and then the restrictions between the upper and lower diagonal parts will be taken care of (second step).

In both steps, the algorithm works on a subset $T$, which itself is partitioned into two subsets $T=T_{1} \cup T_{2}$. Initially, $T_{1}=\varnothing$, and $T_{2}$ is filled with the elements of $Y$; all elements of the partition of $T_{2}$ are singletons. Gradually, subsets will be transferred from $T_{2}$ to $T_{1}$, and in each cycle of the algorithm, the maximal $R$-consistent partition of $T_{1}$ will be determined. If, finally, $T_{2}$ is empty, we fill $T_{2}$ with the elements of $Y^{c}$ and the shifting of subsets to $T_{1}$ continues until $T_{2}$ is empty once again.

At the beginning and at the end of each cycle in the algorithm, the elements of $T$ will be arranged in a ranking that is $R$-isotonic and is also isotonic with respect to $q$ ( $q$-isotonic) in $T_{1}$. This means that, overall, the elements of $T$ are arranged according to the (partial) order generated by the model restrictions, and that, in addition, the elements of $T_{1}$ are arranged isotonically with the weighted averages of the solution blocks they belong to, i.e. the elements of the maximal $R$-consistent partition of $T_{1}$.

Furthermore, for any two members $A, B$ of the current partition of either $T_{1}$ or $T_{2}$, the elements of $A$ are located either all to the right or all to the left of all elements of $B$. In other words, subsets are adjacent and for this reason predicates as "left", "right" and activities as "interchanging", "shifting", and "inserting" will also be used for subsets, in which case they will always be used in relation to the $R$-isotonic and $q$-isotonic ranking of the corresponding elements. In Figure 4.3 a graphical illustration of the steps of the algorithm can be found.

(0) Start

We start with $T_{1}=\varnothing, T_{2}=Y$, a partition of $T_{2}$ into singletons, and an $R$-isotonic ranking of the elements in $T$. Any initial $R$-isotonic ranking will do.

(1) Search for a restriction.

Take the leftmost element $F$ of the current partition of $T_{2}$. Search all elements in $T_{1}$ from right to left for an element that is $R$-constrained by an element of $F$, i.e. for an element $(a, b)$ for which $(a, b) R(x, y)$ holds for some element $(x, y) \in F$. If such an element cannot be found, jump to (3). 


\section{$T_{1}$}

1.

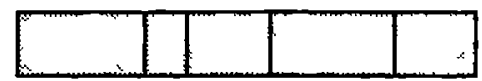

E

2.

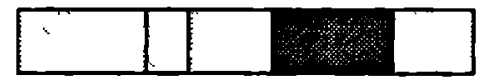

E $\quad F$

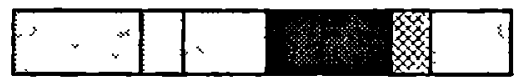

$B$

4.
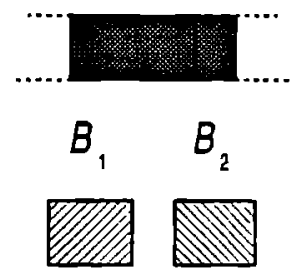

$B$,

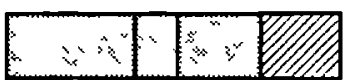

Aाminv

$B_{2}$

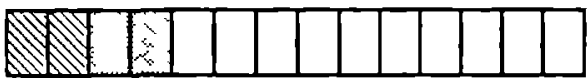

C $D$

5.

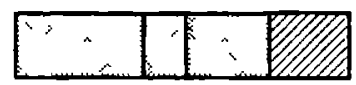

D C

6.

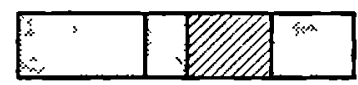

Figure 4.3 - An graphical illustration of the algorthm. 
(2) Check whether the restriction is satisfied by the regressed data.

Suppose $(a, b) \in E$, where $E$ is an element of the maximal $R$-consistent partition of $T_{1}$. If the restriction is satisfied we do not need to modify this partition. If it fails, , i.e. if $q_{E}>q_{F}$ holds, we transfer $F$ from $T_{2}$ to $T_{1}$, and insert $F$ immediately to the right of $E$ in the current ranking. Take the union $B=E \cup F$, and jump to (4).

(3) No violation of a restriction: Adjust the ranking.

Transfer $F$ from $T_{2}$ to $T_{1}$, and insert this subset in the ranking based on the value of $q_{F}$, in such a way that the ranking is $q$-isotonic in $T_{1}$. Jump to (7).

(4) Violation of a restriction: Pool and lookfor an $R$-consistent partition. Examine $B$ to see whether an $R$-consistent partition can be found into subsets $B_{1}, B_{2}$, with $q_{B_{1}}<q_{B_{2}}$, in such a way that there does not exist an $R$-consistent partition of $B_{1}$. This task is described in detail below. If such $B_{1}, B_{2}$ cannot be found, step to (5); if they can be found, $B_{2}$ is partitioned into singletons, and these singletons and all subsets to the right of them in $T_{1}$ are shifted back to $T_{2}$. The ranking is not modified. Call the subset $B_{1}$ the set $D$.

(5) Check whether the ranking is still q-isotonic in $T_{1}$.

Check whether there is a subset $C$ immediately to the left of $D$. If such a $C$ does not exist, jump to (7). If such a $C$ exists, examine $q_{C} \leq q_{D}$. If true, jump to (7). If not, check whether $C$ and $D$ are independent, i.e. check if there are no elements $(x, y) \in C,(v, w) \in D$ for which $(x, y) R(v, w)$ holds. If both sets are not independent, take the union $B=C \cup D$, and go to step (4).

(6) If $C$ and $D$ are independent, they are interchanged in the ranking; go to step (5). Otherwise, step to (7).

(7) Take the next subset of $T_{2}$.

If $T_{2} \neq \varnothing$, jump back to (1). Step to (8) otherwise.

(8) If $Y^{C}=\varnothing$, the algorithm stops. Step to (9) otherwise.

(9) Mirror the partition and ranking of $Y$ in $Y^{C}$.

Find an $R$-consistent partition of the elements in $Y^{C}$ by assigning the element $(x, y)$ to the same subset as the element $(w, v)$ if and only if $(x, y)$ is in the same subset as $(\nu, w)$ in the current $R$-consistent partition of $T_{1}$. Set $T_{2}=Y^{C}$. Arrange the elements in a $q$-isotonic ranking by letting $(y, x)$ precede $(w, v)$ in $T_{2}$ if and only if $(v, w)$ precedes $(x, y)$ in the current $q$-isotonic and $R$-isotonic ranking of the elements in $T_{1}$. Jump back to (1). 
In this algorithm sets are examined for the existence of an $R$-consistent partition in step (4) only. A more detailed discussion of this step follows. In step (4), we look for an $R$-consistent partition of $B$ into subsets $B_{1}, B_{2}$, that is with $q_{B_{1}}<q_{B_{2}}$ holding, in such a way that there does not exist an $R$-consistent partition of $B_{1}$. If this step (4) is reached in a particular cycle of the algorithm, there has always been an initial union of the set $F$ and a set $E$, in the sense of step (2), with $q_{E}>q_{F}$.

We will now first show that an $R$-consistent partition of $B=B_{1} \cup B_{2}$ in step (4) exists if and only if $F \subset B_{1}$.

\section{PROOF}

Since $q_{F}<q_{E}, B=E \cup F$ it follows that $q_{F}<q_{B}$. If an $R$-consistent partition of $B$ exists, then either $F=B_{1}, F=B_{2}, F \subset B_{1}$ or $F \subset B_{2}$.

The partition $B_{1}=F, B_{2}=E$ cannot be $R$-consistent; it violates restrictions, for otherwise we would not have jumped to step (4), but to step (3). On the other hand, the partition $B_{1}=E, B_{2}=F$ cannot be $R$-consistent either, since $q_{E}>q_{F}$. Suppose an $R$-consistent partition of $B$ exists with $F \subset B_{2}$, and $q_{B_{1}}<q_{B}<q_{B_{2}}$. Since $q_{F}<q_{B}$, we have $q_{F}<q_{B_{2}}$ and $q_{B_{2}}<q_{B_{2} \vee F}$. From the latter follows $q_{B_{1}}<q_{B_{2} \vee F}$, which means that an $R$-consistent partition of $B \backslash F=E$ has to exist. This contradicts the requirement that the maximal $R$-consistent partition of $T_{1}$ had been found at the end of the previous cycle. Therefor, if an $R$-consistent partition of $B$ exists, $F$ has to be a proper subset of $B_{1}$.

QED

This result provides the basic rationale for the search for a partition in step (4). A consequence of this result is that if $B$ contains only two elements, there is no need to go through these steps, since an $R$-consistent partition will never be found.

Because of the result just proven, a first candidate for $B_{1}$ is the subset $F$, together with all elements that are $R$-constrained by an element of $F$. If this set forms a suitable $B_{1}$, we are done. If not, i.e. if $q_{B_{1}} \geq q_{B_{2}}$, we will gradually shift elements from $B_{2}$ to $B_{1}$, until we either find a $B_{1}$ with $q_{B_{1}}<q_{B_{2}}$, or have exhausted all possible partitions of $B$, that is, no $R$-consistent partition of $B$ into $B_{1}$ and $B_{2}$ can be found, and we continue as described in step (4) above.

This process is not entirely equivalent to a simple examination of all possible partitions of $B$, due to the fact that we have to take into account the restrictions imposed by $R$. Furthermore, the sequence of transferring elements from $B_{2}$ to $B_{1}$ is rather arbitrary. We have no clear-cut path to an appropriate set $B_{1}$ that cannot be partitioned further. So if we find a $B_{1}$ with $q_{B_{1}}<q_{B_{2}}$, we will have to 
repeat the process with the elements of $B_{1}$. A more formal description of this process follows.

In step (4), the set $B$ will be partitioned into five subsets :

$\begin{array}{ccl}B=A_{0} \cup A_{1} \cup A_{2} \cup A_{3} \cup A_{4} & \\ B_{1} & A_{0} & \text { kernel } \\ & A_{1} & \text { included elements } \\ A_{2} & \text { enforced elements } \\ B_{2} & A_{3} & \text { excluded elements } \\ & A_{4} & \text { prohibited elements }\end{array}$

The set $A_{0}$ contains $F$ and all elements $(x, y) \in B$ for which $(x, y) R(a, b)$ holds for some $(a, b) \in F$. Initially, the remaining elements are allocated to $A_{3}$; they are excluded, i.e. not in $B_{1}$. All other subsets are empty. We set $B_{1}=A_{0}, B_{2}=A_{3}$ and see whether this constitutes and $R$-consistent partition.

If this partition is not $R$-consistent, we will shift elements from $B_{1}$ to $B_{2}$ in order to make the partition an $R$-consistent one. More exactly, we will include into $B_{1}$ formerly excluded elements from $A_{3}$, by moving them to $A_{1}$. In order to satisfy the $R$-consistency of the partition, we have to shift all elements that are $R$-constrained by included elements to $A_{2}$, the subset of enforced elements. We will successively set $B_{1}=A_{0} \cup A_{1} \cup A_{2}, B_{2}=A_{3} \cup A_{4}$ and check whether this is an R-consistent partition.

Within $A_{4}$ we will store elements to prevent the same partition of $B$ from being examined twice, or to prevent useless evaluations. The elements in $A_{4}$ are prohibited; as long as they are in $A_{4}$, they cannot be shifted to $B_{1}$. Elements that have been allocated to $A_{0}$ will not be removed from it, but the contents of other subsets may change in this step.

The shifting of elements corresponds to a systematic evaluation of all allowable partitions of $B$, and examining whether they are R-consistent. To evaluate these partitions in a orderly way, we will take advantage of the current $R$-isotonic ranking of the elements of $B$, by using a pointer and considering the elements to the left and to the right of it. The ranking itself is not modified.

(4.a) The kemel $A_{0}$ consists of $F$ and all elements $(x, y) \in B$ for which $(x, y) R(a, b)$ holds for some $(a, b) \in F . A_{3}$ contains the remaining (excluded) elements of $B$, if any. If $A_{3}=\varnothing$, an $R$-consistent partition of $B$ cannot be found; go to (7). If $A_{3} \neq \varnothing$, the pointer is attached to the leftmost element in $A_{0}$.

(4.b) Evaluate 


$$
q_{B_{1}}<q_{B}<q_{B_{2}} .
$$

If [4.16] is true, an $R$-consistent partition has been found; jump to (4.e). Step to (4.c) if [4.16] is false.

(4.c) Check the elements to the right of the pointer for an excluded element $(a, b) \in A_{3}$, for which there is at least one restriction $(x, y) \in A_{0} \cup A_{1}$ with $(x, y) R(a, b)$. Step to (4.d) if such an element cannot be found. If such an $(a, b)$ can be found, it is transferred to $A_{1}$, and all elements $(x, y) \in A_{3}$ to the left of $(a, b)$ for which $(x, y) R(a, b)$ holds become enforced elements of $B_{1}$; they are moved to $A_{2}$. Attach the pointer to the leftmost element that was transferred from $A_{3}$ in this step (4.c). Go to (4.b).

(4.d) Take the element $(a, b)$ that was most recently added to $A_{1}$. If there is none, we are finished. If there is one, all elements that have been transferred to either $A_{2}$ or $A_{4}$ after $(a, b)$ was moved to $A_{1}$ are transferred back to $A_{3}$. Push $(a, b)$ to $A_{4}$, together with any elements $(x, y)$ to the right of it for which $(a, b) R(x, y)$ holds. Attach the pointer to $(a, b)$. Go to (4.c).

(4.e) The search for a further partitioning of the current $B_{1}$ is prepared. If $A_{1}=\varnothing$, there is no such partition and we step to (4.f). If $A_{1} \neq \varnothing$, all members of $A_{3}$ become prohibited elements and are transferred to $A_{4}$; after this transferral, they will remain prohibited for the rest of this cycle. All elements of $A_{1}$ are shifted back to $A_{3}$, except the element $(a, b)$ that was most recently added to $A_{1}$. The elements currently in $A_{2}$, if any, are moved to $A_{3}$ as well. Scan $A_{3}$ for elements $(x, y)$ for which $(x, y) R(a, b)$ holds, and transfer these to $A_{2}$. If, after these actions, $A_{3}=\varnothing$, we know that an $R$-consistent partition of $B_{1}$ does not exist and step to (4.f). If $A_{1} \neq \varnothing$, the pointer is attached to the leftmost element of $A_{1} \cup A_{2}$ and we go back to (4.b).

(4.f) The elements of $A_{3}$ and $A_{4}$ are transferred as singletons back to $T_{2}$; step to (5).

If, in this step, the subset $B$ can be $R$-consistently partitioned, we have to break $B_{2}$ up into singletons, because we cannot guarantee that an $R$-consistent partition of $B_{2}$ itself does not exist. Transferring the singletons to $T_{2}$, and adding them once again, one by one, to $T_{1}$, is algorithmically the simplest solution to this problem.

Depending on the characteristics of the relation $R$, the algorithm can be speeded up by using the following Lemmas, which we will present without proof. 


\section{LEMMA 4.3}

If there exists a partition $Y=Y_{1} \cup \ldots \cup Y_{n}$, such that there are no elements $(v, w) \in Y_{i},(x, y) \in Y_{,}(i \neq j)$ with $(v, w) R(x, y)$, then the maximal $R$-consistent partition of $Y$ is the union of the subsets $Y_{i}$ or their maximal $R$-consistent partitions, whenever there exists one.

\section{LEMMA 4.4}

If $R, S, R \subseteq S$, are binary relations that generate partial orders on $Y$, then the following relation holds for the isotonic regressions $q_{R}^{*}, q_{S}^{*}$ of the choice proportions $q$ with weights $k$ on $R, S$ respectively:

$$
l_{Y}\left(k ; q_{S}^{*}\right) \leq l_{Y}\left(k ; q_{R}^{*}\right) \leq l_{Y}(k ; q)
$$

\subsubsection{Gebhardt's algorithm}

The algorithm described in 4.3 .2 is functionally equivalent to the second algorithm described by Gebhardt (1970), but by working on subsets rather than on single elements, our algorithm is faster. Furthermore, the new algorithm is more general, since Gebhardt's only accepts asymmetric binary relations $Y$ on $X$.

The major difference between Gebhardt's algorithm and ours however is to be found in step (4.c). Unlike Gebhardt's, our algorithm does not stop once an $R$-consistent partition of $B$ is found, but tries to partition $B_{1}$ further, since no guarantee can be given that an $R$-consistent partition of $B_{1}$ itself cannot be found.

A counterexample will be given. Suppose we have the following elements in $T$ :

$$
\begin{array}{ccccc}
(t, y) & (u, y) & (v, y) & (w, y) & (x, y) \\
k_{\mathrm{ry}}=9 & k_{\mathrm{uy}}=5 & k_{\mathrm{vy}}=2 & k_{\mathrm{wy}}=5 & k_{x y}=1 \\
& & n_{\mathrm{ry}}=n_{\mathrm{uy}}=n_{\mathrm{vy}}=n_{\mathrm{wy}}=n_{\mathrm{xy}}=10 & \\
& (t, y) R(u, y) & (t, y) R(v, y) & (t, y) R(w, y) & (t, y) R(x, y),
\end{array}
$$

in an initial $R$-isotonic ranking

$$
\overline{(t, y)(u, y)(v, y)(w, y)(x, y)} \text {. }
$$




\section{Table 4.2}

History of the algorithm to compute the isotonic regression, applied to the data and restrictions in equation [4.17].

\begin{tabular}{|c|c|c|c|c|c|c|}
\hline Cycle & $T_{\text {, }}$ & & & & & $T_{2}$ \\
\hline 0. & & $\begin{array}{l}\text { ty } \\
.9\end{array}$ & $\begin{array}{r}\text { uy } \\
.5\end{array}$ & $\begin{array}{r}\text { vy } \\
.2\end{array}$ & $\begin{array}{c}\text { wy } \\
.5\end{array}$ & $\begin{array}{l}x y \\
.1\end{array}$ \\
\hline 1. & $\begin{array}{l}\text { ty } \\
.9\end{array}$ & & $\begin{array}{r}\text { uy } \\
.5\end{array}$ & $\begin{array}{r}\text { vy } \\
.2\end{array}$ & $\begin{array}{c}\text { wy } \\
.5\end{array}$ & $\begin{array}{l}x y \\
.1\end{array}$ \\
\hline 2. & ty & $\begin{array}{l}\text { uy } \\
.7\end{array}$ & & $\begin{array}{r}\text { vy } \\
.2\end{array}$ & $\begin{array}{c}\text { wy } \\
.5\end{array}$ & $\begin{array}{l}x y \\
.1\end{array}$ \\
\hline 3. & ty & uy & $\begin{array}{c}\text { vy } \\
.533\end{array}$ & & $\begin{array}{c}\text { wy } \\
.5\end{array}$ & $\begin{array}{l}\text { xy } \\
.1\end{array}$ \\
\hline 4. & ty & uy & vy & $\begin{array}{l}\text { wy } \\
.525\end{array}$ & & $\begin{array}{l}x y \\
.1\end{array}$ \\
\hline 5. & ty & vy & $\begin{array}{l}\text { xy } \\
.4 \\
\end{array}$ & & $\begin{array}{l}\text { uy } \\
.5\end{array}$ & $\begin{array}{l}\text { wy } \\
.5\end{array}$ \\
\hline 6. & ty & vy & $\begin{array}{l}x y \\
.4\end{array}$ & $\begin{array}{l}\text { uy } \\
.5\end{array}$ & & $\begin{array}{r}w y \\
.5\end{array}$ \\
\hline 7. & ty & vy & $\begin{array}{l}x y \\
.4\end{array}$ & $\begin{array}{l}\text { uy } \\
.5\end{array}$ & $\begin{array}{r}\text { wy } \\
.5\end{array}$ & \\
\hline
\end{tabular}

The history of the algorithm, when applied to this problem, can be found in Table 4.2.

At the start of the fifth cycle, $F=\{(x, y)\}$ is the next subset to be transferred to $T_{1}$. At that moment, the current partition of $T_{1}$ is not an $R$-consistent one, since it is a singleton $\{C\}, C=T_{1}$ with $q_{C}=.525$. Let $B=C \cup F$, as described. In step (4), $A_{0}$ has as its elements $\{(t, y),(x, y)\}$. After transferring $(u, y)$ and $(v, y)$ to $A_{l}$ we have 


$$
\begin{aligned}
& q_{A}=.44 \\
& q_{A_{0} \cup A_{1}}=.425
\end{aligned}
$$

so an $R$-consistent partition has been found, with $B_{1}=A_{0} \cup A_{1}$. At this moment, Gebhardt's algorithm would cut short the partitioning process. However, $B_{1}$ itself can be partitioned further:

$$
q_{A_{0} \cup((v, y)\}}=.4
$$

which means the recursivity in step (4) is really needed.

\subsubsection{Application}

Within this section we will lay out the way in which the algorithm described earlier has to be applied if estimates have to satisfy one of the necessary properties in the sense of section 4.2 , conditional on a specified ranking.

\section{Ideal point conditions}

For the ideal point condition $I P$.I, we have $Y=\{z\} \times X$. We can partition $Y$ into couples $Y_{1}=\{(x, z),(z, x)\}$, and use Lemma 4.3. For the ideal point condition $I P .2$, we have $Y \cup Y^{c}=X \times X$, and the relation $R$ based on the inequalities [2.9].

For the remaining properties related to the ideal point, the algorithm needs a minor modification since these properties involve restrictions on probabilities in a BCP system $\langle X, K\rangle$, consisting of the structures $\left\langle X, k_{\imath}\right\rangle\left(i=1, \kappa_{k}\right)$. For this purpose, the relation $Y$ will have to raised to a power, which is determined by the number of structures of binary choice frequencies to be dealt with, $\kappa_{k}$. Each element $(x, y)_{1} \in Y$ will refer to one such structure of binary choice frequency structures $\left\langle X, k_{1}\right\rangle$, and the functions $q_{1}, n_{1}$ have to be redefined accordingly.

\section{Unidimensional unfolding}

For characteristic monotonicity and a given permutation, the relation $R$ is based on the restrictions defined earlier. For bilateral monotonicity and a given permutation, the situation depends on whether the location of the ideal in the unfolded order is known. If its location is unknown, we have a set of relations $R_{1}\left(i=1, \kappa_{X}\right)$, with $\kappa_{X}$ the cardinality of the set $X$. This follows from the fact that the ideal may be closest to or identical with any of the elements of $X$. 
For bilateral/characteristic monotonicity and a given permutation, the situation depends once again on whether the location of the ideal in the unfolded order is known. If it is known, we do not have just one but a multitude of relations $R_{j}$, whose number is determined by the number of permissible patterns of row minima. If the location of the ideal altemative is unknown, we even have a multitude of relations $R_{i j}\left(i=1, \kappa_{x}\right)$ to examine.

\section{Stochastic transitivity}

Lemma 4.3 comes in handy when estimates satisfying stochastic acyclicity have to be found conditional on a ranking known to be weak stochastic. Each $Y_{i}\left(i=1,\left(\kappa_{x}\left(\kappa_{x}-1\right)\right) / 2\right)$ consists of one set of pairs $\{(x, y),(y, x)\}$. If $y$ precedes $x$ in this permutation, then $(y, x) R(x, y)$. If $q_{x x} \leq q_{x y}$, we have found the maximal $R$-consistent partition of this subset, which contains two singletons. If $q_{y x}>q_{x y}$, there does not exist an $R$-consistent partition, and $q_{Y_{1}}=q_{x y}=q_{y x}=.5$.

For the remaining cases of stochastic transitivity, Lemma 4.3 cannot be applied. For each couple of pairs $(x, y),(y, x)$ assign $(y, x)$ to $Y$ and $(x, y)$ to $Y^{c}$ if and only if the elements are sequenced as $\overrightarrow{y x}$ in the ranking.

The restrictions for a moderate stochastic ranking present a problem. They were defined in terms of the location of triples of alternatives in a ranking [4.7]. For each triple, restricted maximum likelihood estimates can be found easily. One simply compares the isotonic regression $q_{R_{1}}$ of $q$ on the relation $R_{1}$

$$
(y, x) R_{1}(x, y) \quad(x, w) R_{1}(w, x) \quad(y, w) R_{1}(x, w)
$$

with the isotonic regression $q_{R_{2}}^{*}$ of $q$ on the relation $R_{2}$

$$
(y, x) R_{2}(x, y) \quad(x, w) R_{2}(w, x) \quad(y, w) R_{2}(y, x)
$$

and takes the isotonic regression that result in the higher likelihood. The problem is that we have to consider the intersection over all triples of the subsets of the parameter space that correspond to these restrictions. This implies that we have to consider a set of relations $R_{1}\left(i=1,2^{\prime}\right)$, with

$$
t=\frac{\kappa_{x}\left(\kappa_{x}-1\right)\left(\kappa_{x}-2\right)}{6}
$$


to find maximum likelihood estimates conditional on one MS ranking. Since this would lead to an incredibly time-consuming search even for a moderate number of elements in $X$, an alternative algorithm is used. Dykstra (1985) has proposed an iterative algorithm that can be modified for our purpose.

This iterative MS-algorithm calculates maximum likelihood estimates of choice probabilities conditional on a moderate stochastic ranking. In each cycle $n$ of this algorithm, for each triple $j$ of $t$ triples consecutively, a function $o_{n, j}^{*}$ is calculated. This computation is based on a comparison of two isotonic regressions of a function $o_{n, 1}$ with weights $k$ on the relations $R_{1}, R_{2}$ as in [4.21-4.22] respectively, corresponding to the triple $j$ in the moderate stochastic ranking considered. The function $o_{n, 1}^{*}$ receives its values from the isotonic regression with the higher likelihood:

$$
\begin{array}{cc}
o_{n, J}^{*}=o_{n_{1} \mid R_{1}}^{*} & \text { if } l_{x}\left(k ; o_{n, j \mid R_{1}}^{*}\right) \geq l_{x}\left(k ; o_{n, \mid<R_{2}}^{*}\right) \\
\dot{o}_{n, J}^{*}=o_{n, J \mid R_{2}}^{*} & \text { otherwise. }
\end{array}
$$

Define the functions

$$
\begin{aligned}
& s_{1, j}(x, y)=1 \\
& s_{n, j}(x, y)=\frac{o_{n-1, j}^{*}(x, y)}{o_{n-1, j}(x, y)} \quad(n \neq 1) .
\end{aligned}
$$

The functions $o_{n, j}$ are defined as follows

$$
\begin{array}{ll}
o_{1,1}(x, y)=q_{n y} \\
o_{n, 1}(x, y)=o_{n-1,1}^{*}(x, y) s_{n, 1}(x, y) & (n \neq 1) \\
o_{n, y}(x, y)=o_{n, y-1}^{\cdot}(x, y) s_{n, j}(x, y) & (j \neq 1),
\end{array}
$$

where $q_{x y}$ stands for the choice proportion, as before.

Dykstra assumed that this iterative procedure, which he proposed within the more general context of $I$-projections, always converges correctly, but he did not provide rigorous proof for his conjecture. Our iterative MS-algorithm did converge for practically all sets of frequencies we tried it on so far. On very rare occasions, the algorithm starts to oscillate close to the solution. This can be monitored by observing the sum of the $\log \left(s_{n, 1}\right)$. If the addition of this sum 
on cycle $n-1$ to the sum on cycle $n$ reaches zero, one has to resort to the general algorithm presented earlier, performing the extensive search over all possible relations.

\subsection{A BRANCH SEARCH STRATEGY}

In the previous section we discussed the problem of obtaining estimates conditional on a partial order on pairs in $X$. For most properties, this partial order itself was conditional on a ranking of the feasible alternatives in $X$. If this ranking is known, these estimates can be obtained by an isotonic regression of the choice proportions, with the number of presentations as weights.

In order to find the estimates under, say, strong stochastic transitivity, without the $D$-isotonic ranking being known a priori, all possible strong stochastic rankings of the altematives in $X$ have to be considered. For each such ranking, we can obtain conditional maximum likelihood estimates, and the value of the likelihood function be computed. If these values are compared one by one, and the ranking(s) for which the likelihood is maximized is selected, the corresponding estimates will be maximum likelihood estimates under strong stochastic transitivity.

The latter implies that the problem of finding maximum likelihood estimates can be reduced to a seriation problem for most properties, with the likelihood as the objective function. However, even for a small number of elements in $X$, an exhaustive search through all $\kappa_{\chi}$ ! rankings in $R(X)$ is a cumbersome, if not impossible task.

One of the problems we are considering, finding maximum likelihood estimates under stochastic acyclicity, has enjoyed a considerable amount of attention in the literature, where it is generally known as the problem of finding maximum likelihood paired comparison rankings.

Flueck and Korsh (1975) have presented a proof for the equivalency of the ranking and the binary preference relation viewpoint in searching for a maximum likelihood weak stochastic ranking. They also focused on non-forced paired comparisons (where ties are allowed), and studied several other binary preference relations. Flueck and Korsh (1974) showed that an efficient branch search algorithm can be used to find a maximum likelihood preference relation. The algorithm we present later on is conceived in their line of thought.

Hubert and Schultz (1975) have explicitly recognized the relation between the problem of finding a maximum likelihood paired comparisons ranking and the quadratic assignment problem developed in operations research. Since the former can be regarded as a special case of the latter, a solution can be found using any of the computational procedures developed for quadratic assignment problems. Among these procedures are branch and bound techniques, which 
Level 5

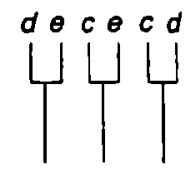

Level 4

Level 3

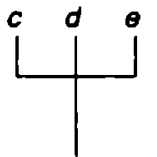

b
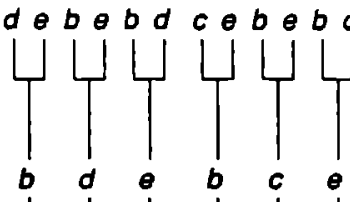

$c d b d b c$ de c e c
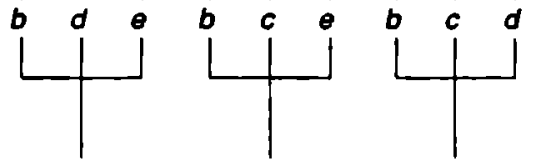

$b$ c d
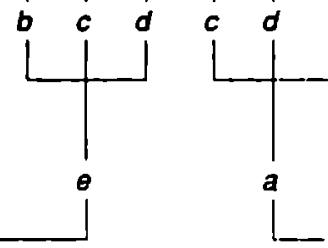

Level 2

a

Level 1

Figure 4.4-Partial view of a ranking tree for a five-element set $X=\{a, b, c, d, e\}$.

generate optimal solutions, and methods generating suboptimal solutions, as the techniques based on the pairwise exchange heuristic. Yet Hubert and Schultz admit that routines that take into account the special characteristics of the likelihood as the objective function and the so-called structure matrix (as does the technique proposed by Flueck and Korsh (1974) may be more efficient.

Other important contributions have been made by and Thompson and Remage (1964), Remage and Thompson (1966), Singh and Thompson (1968), DeCani $(1969,1972)$, and Ranyard (1976).

In the remainder, an algorithm will be presented that can be used for all properties, not just stochastic acyclicity. Its basic principle is the branch search technique, which will be described in the first section. The algorithm itself will be presented in the second. We will consider the application of this technique to the properties we are interested in the third section.

\subsubsection{The branch search principle}

Let $R(X)$ be the set of all rankings $r_{1}(X)$ of the elements in a set $X\left(i=1, \kappa_{X} !\right)$. The branch search algorithm performs a search in $R(X)$ by making moves in a ranking tree. An example of such a tree, for a set of five elements $X=\{a, b, c, d, e\}$, can be found in Figure 4.4. Let $r_{\max }=\kappa_{x}$ be the number of elements in the required ranking. In the example in Figure $4.4, r_{\max }=5$. Each 
path in this tree, from the root through consecutive nodes up to level $r$ corresponds to a subset of $R(X)$. This subset consists of the rankings in which the leftmost $(r-1)$ elements are as specified by the labels on the branches between consecutive nodes. For example, a path along branch $a$ at level 2, branch $b$ at level 3, and branch $c$ at level 4 in Figure 4.4 corresponds to the subset of all rankings in which $\overrightarrow{a b c}$ are the three leftmost elements: $\{\overrightarrow{a b c d e}, \overrightarrow{a b c e d}\}$. Consequently, the root (level 1) corresponds to $R(X)$, and a path up to level $r_{\max }$ corresponds to a singleton, one ranking only.

We will be looking for maximum likelihood rankings. In doing so, it is functionally equivalent and computationally more efficient to minimize the generalized likelihood ratio test statistic. Therefore the following function $m_{x}(k ; g)$ is used:

$$
\begin{aligned}
m_{X}(k ; g) & =\sum_{x, y \in X} m_{x y} \\
& =\sum_{x, y}\left(\ln q_{x y}-\ln g_{x y}\right) k_{x y} .
\end{aligned}
$$

This function $m_{\boldsymbol{x}}(k ; g)$ is equal to minus the $\log$ of the generalized likelihood ratio test statistic

$$
-\ln \left(\frac{l_{x}(k ; q)}{l_{x}(k ; g)}\right)
$$

\subsubsection{The algorithm}

The algorithm first generates an initial, suboptimal solution and evaluates the function $m_{x}(k ; g)$. This suboptimal solution is added as the initial solution to the set of provisional solutions, empty at that time, and the corresponding value of $m_{x}(k ; g)$ is stored as $m_{\mathrm{cul}}$, the cutting criterion. The path through the ranking tree that corresponds to this initial solution is discarded from the tree by eliminating one node at level $r_{\max }$, to prevent the same ranking from being evaluated twice.

The algorithm looks for improvements on the initial solution in the following way. Starting from the root (level 1) it consecutively tries to establish a path in the ranking tree, along branches between nodes up to level $r_{\max }$. From a node at level $r$ branches to nodes at level $r+1$ are examined for feasibility one by one. The devices used for evaluating feasibility may differ, depending on the kind of ranking that is required and the corresponding restrictions on the estimates. One device common to all branch search schemes is to calculate the highest lower bound of the function $m_{x}(k ; g)$ in the subset of $\mathcal{R}(X)$ that corresponds to the node at level $r+1$. Its value can be calculated using the restrictions on the estimates that are shared by all rankings in this subset. 
If the highest lower bound of $m_{x}(k ; g)$ exceeds $m_{\text {aul }}$, the branch to the corresponding node is discarded. No element in this subset of rankings will lead to estimates that increase the likelihood, compared to the ranking(s) in the set of provisional solutions. The algorithm then continues to evaluate the remaining branches to the nodes at level $r+1$ in the same way.

If the highest lower bound does not exceed $m_{\text {cul }}$, the algorithm moves along the branch to the node at level $r+1$ and the process repeats itself there. If, finally, the algorithm arrives at a node at level $r_{\max }$, the corresponding subset contains one ranking only and the highest lower bound is the value of the function $m_{x}(k ; g)$ for the conditionally estimated probabilities. If this value is equal to $m_{\text {enn }}$, the ranking is added to the set of provisional solutions. If the value of $m_{x}(k ; g)$ is strictly smaller than $m_{\text {oul }}$, the set of provisional solutions is emptied, the ranking found is added to it and $m_{e w}$ receives a new value accordingly.

If all branches from a node at level $r$ to the nodes at level $r+1$ have been evaluated, the algorithm backtracks along the path to the node at level $r-1$ and checks whether all branches from this node to nodes at level $r$ have been evaluated. If not, the next branch is examined. Otherwise, the algorithm backtracks to the node at level $r-2$. If, ultimately, the algorithm has backtracked to the root and all branches have been evaluated for feasibility, the current provisional solutions are maximum likelihood solutions, and the corresponding sets of estimates are maximum likelihood estimates.

The branch search principle guarantees that the resulting solution is optimal because it evaluates all possible rankings at least implicitly. If the minimum of the objective function $m_{X}(k ; g)$ in a subset of $R(X)$ is higher than the value of the objective function for a suboptimal solution, we can be sure that the optimal solution is not to be found in this subset.

\subsubsection{Implementation}

In the algorithm we propose, the sequence in which branches are evaluated depends on the initial, suboptimal solution. If two nodes at level $r+1$ correspond to $x$ and $y$, and $x$ precedes $y$ in the initial solution, then the branch to $x$ is evaluated first. If our initial solution is reasonably close to the optimal one, this heuristic may lead to better provisional solutions in an early stage of the search process; lower values of $m_{c u}$ offer the possibility of cutting barren branches closer to the root, thereby reducing the time needed to search the tree.

This strategy of evaluating branches is incorporated in the following algorithm, which offers an easy way to implement the notion of branching and backtracking in a ranking tree.

Generate counters $t_{i}\left(i=1, r_{\text {max }}\right)$, where each $t_{1}$ refers to position $i$ from the left in the ranking. Initially, set each $t_{1}=i$. Each path through the ranking tree is 


\section{Table 4.3}

A possible sequence of counter values, reference rankings and implicitly evaluated subsets of rankings in a branch search procedure.

\begin{tabular}{|c|c|c|c|c|c|c|}
\hline \multicolumn{4}{|c|}{ counters } & \multirow[t]{2}{*}{ reference ranking } & \multirow[t]{2}{*}{$r$} & \multirow[t]{2}{*}{ subset } \\
\hline$t_{1}$ & $t_{2}$ & $t_{3}$ & $t_{4}$ & & & \\
\hline 1 & 2 & 3 & 4 & $\overrightarrow{a b c d}$ & 1 & $\overrightarrow{a \ldots}$ \\
\hline 1 & 2 & 3 & 4 & $\overrightarrow{a b c d}$ & 4 & $\overrightarrow{a b c d}$ \\
\hline 1 & 3 & 3 & 4 & $\overrightarrow{a c b d}$ & 2 & $\overrightarrow{a c \ldots}$ \\
\hline 2 & 2 & 3 & 4 & $\overrightarrow{b a c d}$ & 2 & $\overline{b a \ldots}$ \\
\hline 3 & 3 & 3 & 4 & $\overrightarrow{c b a d}$ & 1 & $\overrightarrow{c \ldots}$ \\
\hline 3 & 4 & 4 & 4 & $\overrightarrow{c d b a}$ & 4 & $\overrightarrow{c d b a}$ \\
\hline
\end{tabular}

represented by one reference ranking and a pointer $r$, whose value indicates that the subset of $R(X)$ under consideration contains the rankings in which the $r$ leftmost elements are as specified in this reference ranking. For example, if the reference ranking is $\overrightarrow{a b c d e}$ with $r=3$, this corresponds to the subset $\{\overrightarrow{a b c d e}, \overrightarrow{a b c e d}\}$. Each counter $t_{t}$ indicates indirectly the number of branches left to be evaluated at level $i$ in the current path in the tree. If $t_{t}=r_{\max }$, there are no more branches to be evaluated at level $i$. If $t_{t}=r_{\max }-1$, there is one candidate left for the position at level $i$. Table 4.3 contains an arbitrarily selected series of counter values for a set of four alternatives $\{a, b, c, d\}$.

Start with the ranking that corresponds to the initial solution, with an empty set of initial solutions, $m_{\mathrm{att}}=\infty$ and $r=1$.

(0) Set $r=1$.

(1) Examine the subset of rankings with the $r$ leftmost elements as specified for the presence of a better provisional solution, using the appropriate devices. If there is none, jump to (4).

(2) Evaluate $r=r_{\max }$. If true, we have a provisional solution. Store it, adapt $m_{\text {aut }}$ accordingly, and jump to (4).

(3) Increment $r$ by one. Go to (1).

(4) Evaluate $t_{r}<r_{\text {max }}$. If true, increment $t$, by one, and insert the element currently at position $t_{r}$ in the reference ranking at position $r$. Jump back to (1). 
(5) Evaluate $r=1$. If true, the algorithm stops, since all rankings have been evaluated. The current provisional solution(s) is (are) maximum likelihood solutions.

(6) Evaluate $r<r_{\text {max }}$. If true, insert the element currently at position $r$ in the reference ranking at the rightmost position $r_{\text {max }}$.

(7) Set $t_{i}=i$ and decrement $r$ by one. Go to (4).

We have mentioned earlier that, depending on the probabilities, several rankings can be $D$-isotonic. This means that, from a ranking point of view, several rankings may lead to equivalent maximum likelihood estimates. The same observation holds for many other properties. If finding the constrained maximum of the likelihood function prevails over discovering optimal rankings, the efficiency of the algorithm can be further improved. In that case the algorithm may be instructed to stop once a provisional solution with $m_{x}(k ; g)=0$ is found. Furthermore, the algorithm can be instructed to discard branches in the ranking tree even if the highest lower bound in the corresponding subset of rankings equals $m_{\text {an }}$. In that case, not all optimal rankings are recovered by the algorithm itself, but they can be easily reconstructed by an examination of the maximum likelihood estimates.

For example, in the extreme case where $k_{x y}=k_{y x}$ holds for all $x, y \in X$, the branch search algorithm would go through an explicit, time-consuming and superfluous evaluation of all $\kappa_{x}$ ! rankings unless one requires strict inequality. With the latter option, all remaining branches can be timely discarded.

For most properties, we have found the efficiency of the algorithm to be sensitive to the sequence in which branches are evaluated, and, even more, to the method used for finding the initial solution. The problem of finding the initial solution will be discussed for each condition separately in the following section.

\subsubsection{Application}

At three places the algorithm has to be welded for each condition separately: in the method for finding the initial ranking, in the restrictions to be used in finding the highest lower bound of the objective function, and in any supplementary devices that can be applied to eliminate branches in the ranking tree.

\section{Ideal point conditions}

The branch search algorithm will only be used for the unilaterally increasing/decreasing condition [2.13-14] and the bilateral condition [2.15], since the 
remaining ideal point properties are not conditional on the unfolded order. How to obtain an initial ranking is not self-evident, but the following heuristic can be used.

Find the smallest choice proportion, say $q_{x y}$. Allocate $x$ to the leftmost position of the ranking. Assign the remaining $y$ to positions in the ranking isotonically with the choice proportions $q_{\mathbf{r}}$.

This heuristic has been described by Greenberg (1965), who refers to a suggestion from Coombs. It works best for binary choice frequency structures generated by a probability structure that satisfies characteristic monotonicity.

For the unilaterally increasing/decreasing property, the bilateral condition and every other property that is conditional on the unfolded order, a second device can be used to eliminate branches. A branch can be discarded if all rankings in the corresponding subset have already been evaluated in their inverted order. This follows from the fact that an unfolded order is a ranking that is monotonic only, not isotonic, with the coordinate values. The inverted ranking leads to the same set of ordinal restrictions. In practice, we will examine a subset of rankings only if it contains at least one ranking that has not yet been evaluated in its inverted order. This can be checked easily, by keeping a record of the branches to nodes at level 2 that have already been examined for feasibility.

\section{Unidimensional unfolding}

For characteristic monotonicity, we also use the heuristic described by Greenberg (1965) to generate the initial provisional solution, and the same additional device to cut branches as described earlier for the ideal point conditions.

For bilateral monotonicity, a maximum likelihood weak stochastic ranking is adopted as the initial solution. For this condition, one ranking tree is not sufficient, because we have to consider the possibility that the ideal is closest to the $1, \ldots, \kappa_{x}$ 'th element in the unfolded order. We do not have to examine all $\kappa_{x}$ ranking trees, because an unfolded order is only monotonically, not isotonically related to the values of the coordinates; if a ranking is an unfolded order, so is the inverted ranking. We will therefore examine $n=\left(\kappa_{x}+\bmod \left[\kappa_{x}, 2\right]\right) / 2$ ranking trees. We start with the ranking tree corresponding to the $n^{\prime}$ th position for the ideal, since this involves the smallest number of restrictions. Overall, this strategy can reduce the number of examinations needed in the remaining ranking trees.

For bilateral/characteristic monotonicity, we proceed in much the same way as for bilateral monotonicity. Similarly, a search will be performed in $n$ ranking trees, to take into account the possible locations of the ideal alternative. For each subset of rankings in one such ranking tree, several patterns of restrictions 
have to be examined, since, speaking in terms of matrices, the minima in each row can be located anywhere in between the main diagonal and the column corresponding to the position closest to the ideal.

To speed up the search, we will first calculate the highest lower bound for the restrictions that are shared by all patterns of row minima (Lemma 4.4). If its value is higher than $m_{\text {cun }}$, we discard the branch. If not, we consecutively start to evaluate all possible pattems of row minima, until eventually a value for the highest lower bound is found that does not exceed $m_{\text {ax. }}$. If there is none, the branch can be discarded. If we finally arrive at the bottom of the ranking tree, an exhaustive search through all possible patterns is performed.

\section{Stochastic transitivity}

For stochastic acyclicity, the $m_{x y}$ in [4.25] are either zero or, if $0<k_{x y}$, equal to

$$
m_{x y}=\left(\ln 2+\ln q_{x y}\right) k_{x y}
$$

Though the branch search algorithm presented earlier was inspired by Flueck and Korsh's (1974) scheme to find maximum likelihood weak stochastic rankings, our method for STA differs from theirs. To find an initial solution, Flueck and Korsh (1974) calculated

$$
s_{x}=\sum_{y \in X} m_{x y}
$$

as in [4.27] and ranked elements isotonically with the values of the $s_{x}$. If this ranking is not unique, they let $x$ precede $y$ in this subset whenever $q_{y z} \leq q_{x y}$.

We propose an altemative procedure. As an initial step, evaluate whether there is an element $x$ that can be assigned to the leftmost position in a $D$-isotonic ranking. This means that $q_{y x} \leq q_{x y}$ has to hold for all the remaining $y$. If there is one, eliminate all branches from the root to the nodes corresponding to the remaining elements, and continue the search for the next to leftmost element.

This search stops if one succeeds in arranging all elements, or if no element can be ailocated this way to the next position in the ranking. In the latter case, a similar search is started for the rightmost element in the $D$-isotonic ranking. After having arranged as many elements as possible at the rightmost positions, similarly adapt the ranking tree to prevent needless examinations, i.e. cut all branches at the top of the tree that correspond to subsets with different elements at the rightmost positions. The elements between those fixed at the leftmost 
and the rightmost positions can be arranged in any order to produce an initial solution. Usually, this initial cutting procedure results in a good suboptimal solution and a substantial reduction of the size of ranking tree.

This algorithm is extremely fast. After the initial cutting process, the $m_{x y}$ have to calculated only once, and for a subset of $X \times X$ only. Only a series of simple comparisons and additions remains to be made.

For a moderate stochastic ranking, a maximum likelihood weak stochastic ranking is used as the initial solution. For each subset of rankings, we first calculate the highest lower bound under the restrictions for a wS ranking [4.5] (cf. Lemma 4.4). Since the $m_{x y}$ [4.27] can be calculated before the actual branch search, this lower bound is obtained through a series of simple additions, which results in a much easier and fasterprocedure than an exclusive use of the iterative MS-algorithm presented earlier. If the value of the highest lower bound exceeds the current value of $m_{\text {cul }}$, the branch is discarded, if not, the total set of restrictions is used to calculate the highest lower bound under the restrictions for an MS ranking.

For a strong stochastic ranking (strong stochastic transitivity), a maximum likelihood weak stochastic ranking is also used as the initial solution. Here also, we first calculate the highest lower bound under $D$-isotonicity.

\subsubsection{Extensions}

The strategy for finding maximum likelihood weak, moderate, or strong stochastic rankings, or unfolded order under CM, BM or BCM can be a very efficient data-analytic tool itself. Elsewhere (Bossuyt \& Roskam, 1987a, 1989-a) we have presented an collection of probabilistic unfolding techniques that is based on the search for a maximum likelihood unfolded order under characteristic monotonicity or midpoint monotonicity (a superset of the restrictions of $\mathrm{CM}$ ).

The algorithms presented in this chapter have been implemented in a FORTRAN program called PSTRIX. This program runs on an IBM-compatible PC under MS-DOS or PC-DOS (version 2.0 or higher), but it can be (and has been) easily ported to other systems. The time needed to find maximum likelihood rankings is acceptable, even with a modest CPU and without the help of a floating-point coprocessor. Appendix A contains listings of major subroutines of this program. 


\section{5 TESTING ORDINAL RESTRICTIONS ON BINOMIAL PARAMETERS}

The preceding subsections contain a description of a strategy to compute maximum likelihood estimates of binary choice probabilities under partial order restrictions. The ultimate aim of this strategy is the construction of statistical tests for properties of BCP structures and BCP systems.

In the literature, only statistical tests of the stochastic transitivity conditions have been a topic of concern. If all pairs have been presented only once, the search for a maximum likelihood $D$-isotonic ranking and maximum likelihood estimates under stochastic acyclicity, coincides with the search for Slater's $i$. This statistic is based on the minimum number of inconsistencies for a structure of choices that can be achieved in the set of all rankings. The resulting rankings are called nearest adjoining orders. Slater (1962) proposed this statistic in a reaction against Kendall and Smith's statistic $d$, which was based on the number of circular triads (Kendall and Smith, 1939; cf. Kendall, 1955). Several methods for calculating Slater's $i$ have been proposed (e.g. Phillips, 1967, 1969; Smith \& Payne, 1974; Ranyard, 1976).

Bezembinder (1981) called Slater's and Kendall's indices 'measures of intemal consistency', and compared them with measures of external inconsistency, which evaluate inconsistency with respect to a given linear order. He suggested that both forms of inconsistency should be clearly distinguished and measured independently. We do not entirely agree with this point of view. In general, it is not true that measures of intemal inconsistency are not related to any particular linear order, since most measures can be regarded as the optimum with respect to the set of all possible linear orders, as has been described in the previous subsections. Our method, for example, is capable of calculating the likelihood for a particular ranking thought to be weak stochastic (in which case it becomes a measure of extemal inconsistency), but maximum likelihood estimates under stochastic acyclicity (internal consistency) can be found by performing a search in the set of all weak stochastic rankings. In that case, a likelihood ratio based test statistic serves as an index of internal consistency.

\subsubsection{A generalized likelihood ratio test}

In the algorithm we proposed, the function $m_{x}(k ; g)$ was used as the objective function. It is based on minus the logarithm of the generalized likelihood ratio test statistic and its maximum can be used in a statistical test: The hypothesis $\mathscr{H}_{0}$ that a particular property is satisfied by the structure of choice probabilities that generated the choices will be rejected in favor of the hypothesis $\mathcal{H}_{1}$ that the 
contrary is true, if $m_{x}(k ; g)$ is sufficiently large. To build at test with a size $\alpha$, we need to determine the critical region by specifying a value $\zeta_{1-\alpha}$, based on the distribution function of $m_{x}(k ; g)$ if $\mathscr{H}_{0}$ is true.

Among the main reasons for the likelihood ratio principle's popularity are the attractive asymptotic properties tests derived by this method possess. Unfortunately, it seems that we will not be able to profit from these properties in our problem.

To our knowledge, testing partial order restrictions on binomial probabilities has not been a topic of interest in the statistical literature. Yet testing the equality of (simply) ordered parameters has been studied by a number of authors. Barlow, Bartholomew, Bremner and Brunk (1972) and Robertson, Wright and Dykstra (1988) have summarized most of the results. The majority of the approaches they covered can be traced back to a test on the equality of ordered means of normal distributions.

Suppose a set $Y$ of $k$ series of $n_{i}$ values $y_{11}$ each $\left(i=1, k, j=1, n_{i}\right)$ from normal distributions with respective sample means $\bar{y}_{1}$, have been observed. Suppose that the variances of these normal distributions are known. Consider the hypothesis $\mathcal{H}_{0}$ that all means are equal, versus the alternative hypothesis $\mathcal{H}_{1}$ that they are not, yet satisfy the (partial) order restrictions $R$.

Define two functions $n$ and $y$ on the power set of $Y$, such that $n_{\varnothing}=0$ and $y_{\varnothing}=0$, and for each nonempty subset $B \subseteq Y$,

$$
\begin{aligned}
& n_{B}=\sum_{\bar{y}_{i} \in B} n_{i} \\
& \bar{y}_{B}=\frac{\sum_{\bar{y}_{1} \in B} n_{i} \bar{y}_{i}}{n_{B}} .
\end{aligned}
$$

Suppose that the maximal $R$-consistent partition of the set of sample means $Y$ consists of elements $B_{i}(i=1, l)$ and consider the test statistic

$$
\bar{X}_{k}^{2}=\sum_{i=1}^{l} \frac{n_{B_{i}}}{\sigma^{2}}\left(\bar{y}_{B_{i}}-\bar{y}_{Y}\right)^{2}
$$

Let $\bar{X}_{k}^{2}=0$ if an $R$-consistent partition of $Y$ does not exist.

In this particular case, an asymptotic justification for the use of this statistic is also lacking, yet there exists a proof for the following theorem (Barlow, Bartholomew, Bremner \& Brunk, 1972; Robertson, Wright and Dykstra, 1988). 


\section{THEOREM 4.2}

If $\mathcal{H}_{0}$ is true, then

$$
\begin{aligned}
& \operatorname{Pr}\left[\overline{\mathrm{X}}_{k}^{2} \geq a\right]=\sum_{l=2}^{k} \bar{P}(l, k) \operatorname{Pr}\left[\mathrm{X}_{l-1}^{2} \geq a\right] \\
& \operatorname{Pr}\left[\overline{\mathrm{X}}_{k}^{2}=0\right]=\bar{P}(1, k)
\end{aligned}
$$

where $\bar{P}(l, k)(1<l)$ is the probability that an $R$-consistent partition of exactly 1 subsets exists, $\bar{P}(1, k)$ is the probability that such a partition cannot be found, and $\mathrm{X}_{v}^{2}$ stands for a random variable having a chi-square distribution with $\vee$ degrees of freedom.

Testing the equality of ordered parameters is of little interest for our purpose however. A more interesting test involves the hypothesis $\mathscr{H}_{0}$ that all means satisfy the order restrictions, versus the alternative hypothesis $\mathscr{H}_{1}$ that they do not, and the test statistic

$$
\tilde{X}_{k}^{2}=\sum_{i=1}^{l} \sum_{\bar{y}, \in B_{1}} \frac{n_{B_{1}}}{\sigma_{1}^{2}}\left(\bar{y}_{B_{1}}-\bar{y}_{1}\right)^{2}
$$

where the summation ranges over all solution blocks $B_{i}$, and all sample means

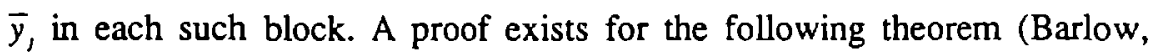
Bartholomew, Bremner \& Brunk, 1972; Robertson, Wright and Dykstra, 1988).

THEOREM 4.3

If $\mathcal{H}_{0}$ is true, then

$$
\begin{aligned}
& \operatorname{Pr}\left[\tilde{\mathrm{X}}_{k}^{2} \geq a\right]=\sum_{l=1}^{k-1} \tilde{P}(l, k) \operatorname{Pr}\left[\mathrm{X}_{k-1}^{2} \geq a\right] \\
& \operatorname{Pr}\left[\tilde{\mathrm{X}}_{k}^{2}=0\right]=\tilde{P}(k, k)
\end{aligned}
$$

where the $\bar{P}(l, k)$ and $\mathrm{X}^{2}$ have the same interpretation as $\bar{P}(l, k)$ and $\mathrm{X}_{\mathrm{v}}^{2}$ in Theorem 4.2 .

Both in Theorem 4.2 and Theorem 4.3, the density function of the test statistic is a weighted sum of well-known parametric densities. Attractive though this may seem, a problem resides in the determination of the probabilities $\bar{P}(l, k)$. Robertson, Wright and Dykstra (1988) summarized how the probabilities $\bar{P}(l, k)$ can be calculated for some simple and small relations $R$, yet their expressions become frighteningly cumbersome as the complexity of $R$ increases. 


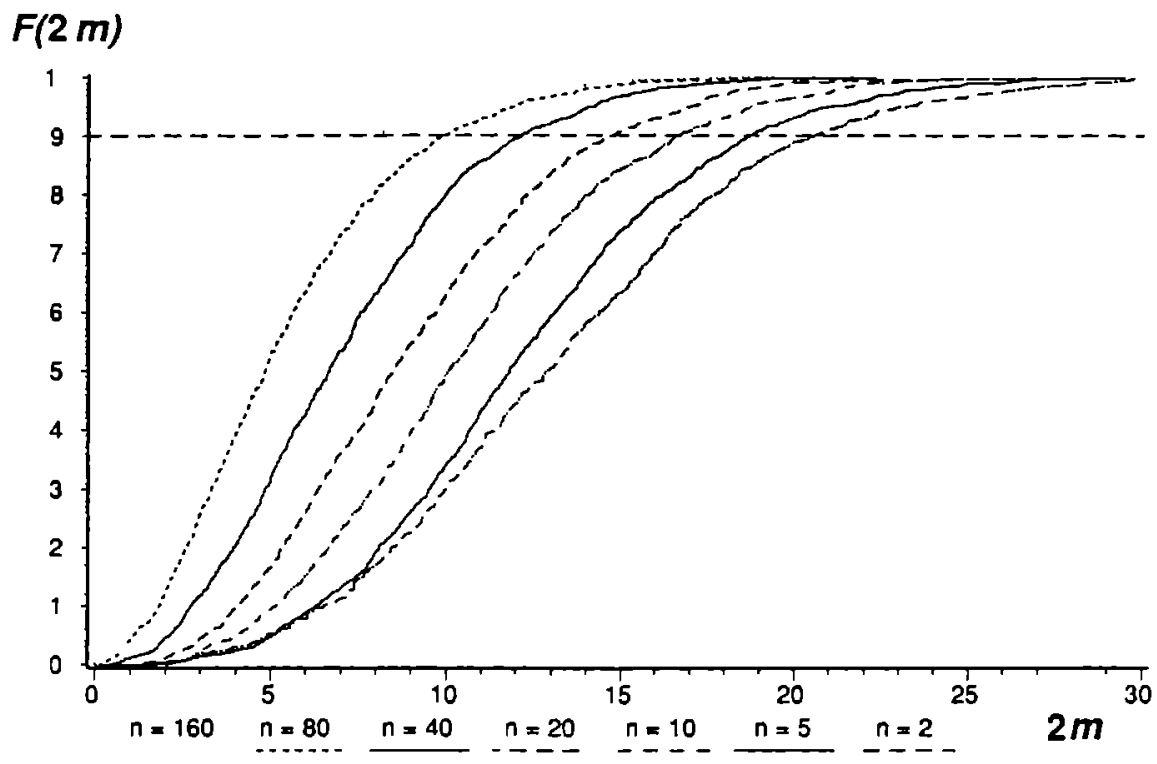

Figure 4.5-Sample cumulatve distribution functions for the generalized likelihood ratıo test statistic under SST.

Even if we had found ways of circumventing the difficulties in computing probabilities $\bar{P}(l, k)$ for our properties, we would still have had to face the problem that we are dealing with non-normal distributions. The choice proportions $q_{x y}$ will be approximately normal by the central limit theorem, but this is of little or no help if our observations involve extreme choice proportions and small samples sizes.

\subsubsection{A Monte Carlo approach}

Having taken into account these statistical obstacles, we decided to rely on a Monte Carlo approach for a non-parametric estimation of the distribution function of the test statistic under the null hypothesis. For a given property we randomly generate a structure of choice probabilities that satisfies the property. Taking each such probability as the second parameter of a binomial distribution, and taking the first parameter (the number of presentations) as a design constraint, we randomly and independently sample a structure of binomial deviates from these distributions. For each such structure we calculate maximum likelihood estimates under the condition studied, and compute the value of -2 $\log$ likelihood ratio test statistic $2 m$. There is no justification in using $2 m$ rather 
than $m$ except for a faint reference to the asymptotic properties of this test in other circumstances. We repeat this Monte Carlo simulation $n$ times, in each simulation calculating the statistic $2 m_{i}(i=1, n)$.

We define the critical region for a test of approximate size $\alpha$ by estimating the $1-\alpha$ quantile of the cumulative distribution function $\zeta_{1-\alpha}$ nonparametrically using order statistics $D_{1}, \ldots, D_{n}$ corresponding to the random sample $2 m_{1}, \ldots, 2 m_{n}$ :

$$
\begin{aligned}
\zeta_{1-\alpha} & =D_{1-\alpha}^{n} \\
& =(1-h) D_{j}+h D_{j+1} \\
(j= & \operatorname{int}[(1-\alpha)(n-1)+1]) \\
(h= & \operatorname{frac}[(1-\alpha)(n-1)+1]),
\end{aligned}
$$

where int $(x)$ is a function truncating the decimal portion of the value of its argument, such that the integer portion of the value of $x$ remains, and $\operatorname{frac}(x)$ is a function returning the decimal portion of the value of its argument, or the remainder of $x / 1$.

This is the maximum likelihood estimator. It is consistent but biased. Asymptotically $D_{1-\alpha}^{n}$ is distributed as a normal distribution with mean $\zeta_{1-\alpha}$ and variance

$$
\frac{(1-\alpha) \alpha}{n\left[f\left(\zeta_{1-\alpha}\right]^{2}\right.}
$$

(Mood, Graybill \& Boes, 1974). This may serve as a guide in determining the sample size needed.

To generate a structure of probabilities that satisfies a particular property, we sample from a uniform distribution over the interval $[0,1]([0.5,1]$ for the stochastic transitivity conditions) using a pseudo random number generator, and assign the sampled values isotonically to a ranking drawn at random from the set of all rankings of probabilities known to be isotonic with respect to the partial order that corresponds to this property. To calculate the binomial deviates, we use an algorithm proposed by Ahrens \& Dieter $(1974,1980)$.

The sampling of a ranking is based on the construction of a random path in a ranking tree. This ranking tree corresponds to the set of all possible rankings of binomial probabilities. We delete all branches in this tree except those that are $R$-isotonic with respect to the property studied. We then build a random path in this mutilated tree, starting from the root, at each node making a random choice between the branches. 
In Figure 4.5 sample cumulative distribution functions are shown for series of 1000 Monte Carlo simulations under strong stochastic transitivity. The simulations were run for a set of eight altematives, and in each series a distinct first parameter of the binomial distributions (the number of trials) was used. The horizontal dashed line can be used to derive the value of $D_{90}$. This value clearly decreases as the first parameter of the binomial distributions increases.

Though this Monte Carlo test strategy has the advantage of a definition of the critical region that takes into account the number of couples and the number of presentations, we have to pay for this advantage in terms of computing time. Yet for a small to moderate number of altematives, these calculations are easy to perform for most properties. The only property that presents a problem in this respect is bilateral/characteristic monotonicity. Calculation times for a set of 100 structures under BCM can quickly run up to several hours on a PC with a modest CPU.

For the equality restrictions, we can perform exact a priori calculations of the cumulative distribution function of the generalized likelihood ratio test statistic. Assume we intend to sample values $k_{v w}, k_{x y}$ from binomial distributions $\left(n_{v w}, p_{v w}\right)$ $\left(n_{x y}, p_{x y}\right)$ respectively, with the first parameter in each distribution known, the second unknown, with a uniform prior distribution. Assume we know the equality $p_{v w}=p_{x y}$ to hold. In that case the joint probability of a pair of frequencies $k_{v w}, k_{x y}$ can be computed:

$$
\begin{aligned}
\operatorname{Pr}\left[k_{v w}, k_{x y} \mid n_{v w}, n_{x y}\right] & =\int_{0}^{1}\left(\begin{array}{l}
n_{v w} \\
k_{w w}
\end{array}\right)\left(\begin{array}{l}
n_{x y} \\
k_{x y}
\end{array}\right) p^{\left(k_{w}+k_{x y}\right)}(1-p)^{\left(k_{x y}+k_{y x}\right)} d p \\
& =\left(\begin{array}{l}
n_{w w} \\
k_{v w}
\end{array}\right)\left(\begin{array}{l}
n_{x y} \\
k_{x y}
\end{array}\right) \frac{\left(k_{w y}+k_{x y}\right) !\left(k_{w y}+k_{y z}\right) !}{\left(n_{v w}+n_{x y}+1\right) !} .
\end{aligned}
$$

By computing this probability and the value of test statistic for all possible pairs of frequencies, we can arrive at an exact calculation of the cumulative distribution function of the generalized likelihood ratio test statistic. A test based on this distribution is comparable to Rice's "conditional binomial exact test" (CBET, Rice, 1988). Compared to Rice's test, ours is an unconditional test, since we want to compute the critical region a priori to the actual computation of the statistic.

A similar exact approach can be utilized to calculate the distribution of the test statistic in the case of a small number of inequality restrictions. For an example, assume once more that values $k_{\mathrm{w} w}, k_{x y}$ from binomial distributions $\left(n_{v w}, p_{w w}\right)\left(n_{x y}, p_{x y}\right)$ respectively are sampled, with in each distribution the first 
parameter known, the second unknown, with a uniform prior distribution. Assume that the inequality $p_{v m} \geq p_{x y}$ is known to hold. In that case the joint probability of a pair of frequencies $k_{v w}, k_{x y}$ is equal to:

$$
\operatorname{PT}\left[k_{v w}, k_{x y} \mid n_{w w}, n_{x y}\right]=\int_{0}^{1}\left(\begin{array}{l}
n_{v w} \\
k_{w w}
\end{array}\right) p^{k_{m}}(1-p)^{k-} \int_{0}^{p}\left(\begin{array}{l}
n_{x y} \\
k_{x y}
\end{array}\right) q^{k_{y y}}(1-q)^{k_{x y}} d q d p .
$$

The computation of the cumulative distribution function can proceed in the same way as for the hypothesis of equality.

The exact calculation the distribution of the likelihood ratio test statistic under the unilaterally increasing (or decreasing condition) by using equation [4.36] is computationally laborious. However, we can use a Monte Carlo approach similar to the ones discussed earlier.

On some occasions though we will be more interested in testing three hypotheses: The hypothesis that the decreasing condition is satisfied, the hypothesis that the increasing condition is satisfied, and the hypothesis that neither is satisfied. For this purpose, we will calculate maximum likelihood estimates under the unilaterally decreasing and increasing condition respectively, and the likelihood ratio test statistic using the contributions

$$
m_{x y}=\left(\ln q_{x y \mid \cup D}^{*}-\ln q_{x y \mid U 1}^{*}\right) k_{x y},
$$

where

$$
\ln q_{x y \mid \text { UD }}^{*} \ln q_{x y \mid U 1}^{*}
$$

stand for the isotonic regression under the unilaterally decreasing and increasing condition respectively.

Using a related Monte Carlo strategy, we can sample binomial deviates from a set of structures of choice probabilities, with the probabilities for each structure drawn at random from a uniform distribution over the parameter space. We will reject the hypothesis that neither the increasing or decreasing condition is satisfied if the value of the test statistic lies in the lower or higher critical region of the sample cumulative distribution function.

\subsection{EXAMPLES}

In this section the evaluation strategy will be applied to some models of data published in the literature. 
Table 4.4

A comparison of test statistics and statisucal decisions of the authors and as reported by Tversky (1969).

\begin{tabular}{lcccc}
\hline & Statistic 1 & Decision 1 & Statistic 2 & Decision 2 \\
\hline Subject 1 & 11.82 & $\ldots *$ & 9.33 & $\ldots$ \\
Subject 2 & 7.84 & $\ldots$ & 4.46 & $\ldots$ \\
Subject 3 & 6.02 & $\ldots$ & 6.02 & $\ldots$ \\
Subject 4 & 15.94 & $\ldots$ & 2.63 & $\ldots$ \\
Subject 5 & 5.18 &. & 3.86 & $\ldots$ \\
Subject 6 & 7.36 & $\ldots$ & 7.26 & $\ldots$ \\
Subject 7 & .40 &. &.$\ldots$ &. \\
Subject 8 & .00 &. & .00 &.
\end{tabular}

Note - Statistic $1=$ calculated by Tversky, Statistic $2=$ calculated by the present author, Decision $1=$ statistical decisions made by Tversky, Decision $2=$ decisions made by the present author;

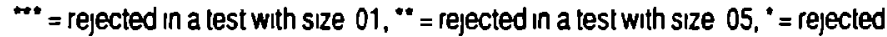
in a test with size $10, \cdot=$ not rejected in a test with size 10 .

\subsubsection{Stochastic acyclicity}

In a paper which appeared some twenty years ago, Tversky (1969) presented a series of demonstrations in order to support his assertion that choices can be consistently intransitive under certain experimental conditions. This paper has become one of the most frequently quoted in the psychological literature on choice and decision making.

In Tversky's first demonstration, eight subjects choose 20 times from all couples out of five gambles. Gambles differed both in the probability of winning - which was presented using shaded circle segments, not numerically - and in the payoff. The five gambles could be simply ordered isotonically with the expected values.

The choice proportions obtained this way contained several stochastic cycli. Tversky presented a statistical test, for which he acknowledged the assistance of David H. Krantz, which is closely related to our approach of testing stochastic acyclicity. Tversky made a ranking of the gambles, isotonic with their expected values, and assumed this to be the underlying weak stochastic ranking. He then calculated the restricted maximum likelihood estimates under stochastic acyclicity, conditional on this ranking. Tversky presented these estimates as maximum likelihood estimates under weak stochastic transitivity, although this condition is unmistakably violated in several of his structures of estimates. 


\section{Table 4.5}

Choice proportions reported by Rumelhart \& Greeno (1971)

\begin{tabular}{cccccccccc}
\hline & $C Y$ & $B B$ & $J U$ & $A J F$ & $C d G$ & $E T$ & $H W$ & $S L$ & $L B J$ \\
$C Y$ & .500 & .406 & .248 & .329 & .329 & .308 & .265 & .261 & .218 \\
$B B$ & .594 & .500 & .470 & .427 & .402 & .286 & .333 & .205 & .261 \\
$J U$ & .752 & .530 & .500 & .491 & .380 & .368 & .299 & .261 & .252 \\
$A J F$ & .671 & .573 & .509 & .500 & .410 & .393 & .316 & .303 & .235 \\
$C d G$ & .671 & .598 & .620 & .590 & .500 & .521 & .410 & .513 & .303 \\
$E T$ & .692 & .714 & .632 & .607 & .479 & .500 & .479 & .372 & .316 \\
$H W$ & .735 & .667 & .701 & .684 & .590 & .521 & .500 & .521 & .321 \\
$S L$ & .739 & .795 & .739 & .697 & .487 & .628 & .479 & .500 & .393 \\
LBJ & .782 & .739 & .748 & .765 & .697 & .684 & .679 & .607 & .500 \\
\hline
\end{tabular}

Note - The row and column elements are ordered according to the maximum likelihood strong stochas $\downarrow c$ ranking.

For a statistical test of the stochastic intransitivities, Tversky used the generalized likelihood ratio test statistic. Tversky assumed that this statistic has a chi-square distribution for a large sample size, with a number of degrees of freedom that equals the number of constrained parameters (the number of probabilities to be estimated minus the number of solution blocks). Tversky's values of the likelihood ratio test statistic, and the statistical decisions he made in tests with varying size, can be found in Table 4.4, where they are labelled Statistic 1 and Decision 1 respectively.

Despite the provocative and valuable content of this paper on the subject of intransitivities in human choices, Tversky's testing procedure errs in three important ways. First, maximum likelihood estimates under weak stochastic transitivity do not always exist. Estimates Tversky presented as such clearly did not satisfy this condition. Second, to test stochastic intransitivities, one cannot simply examine the ranking that is isotonic with the expected values of the gambles. Even in 1969, there was ample evidence that expected value was not always the criterion individual choices were guided by. Instead, Tversky should have considered the set of all rankings of the five gambles. Third, the generalized likelihood ratio test statistic definitely does not have the distribution attributed to it by Tversky, even if the sample size had been considerably higher than 20.

We used our evaluation strategy, including the branch search in the ranking tree, and calculated the generalized likelihood ratio test statistic. The values of the statistic for the eight subjects, and the outcomes of a statistical test of the hypothesis that the subjects' choice behavior satisfied stochastic acyclicity can also be found in Table 4.4. The critical region was defined using quantiles 
Table 4.6

Maximum likelihood estimates under strong stochastic transitivity, based on data in Table 4.5.

\begin{tabular}{cccccccccc}
\hline & $C Y$ & $B B$ & $J U$ & $A J F$ & $C d G$ & $E T$ & $H W$ & $S L$ & $L B J$ \\
$C Y$ & .500 & .406 & .303 & .329 & .303 & .303 & .265 & .242 & .218 \\
$B B$ & .594 & .500 & .470 & .427 & .391 & .306 & .306 & .242 & .242 \\
$J U$ & .697 & .530 & .500 & .491 & .391 & .368 & .306 & .261 & .244 \\
$A J F$ & .697 & .573 & .509 & .500 & .410 & .393 & .316 & .303 & .244 \\
$C d G$ & .697 & .609 & .609 & .590 & .500 & .500 & .432 & .432 & .303 \\
$E T$ & .697 & .694 & .632 & .607 & .500 & .500 & .479 & .432 & .316 \\
$H W$ & .735 & .694 & .694 & .684 & .568 & .521 & .500 & .500 & .321 \\
$S L$ & .758 & .758 & .739 & .697 & .568 & .568 & .500 & .500 & .393 \\
LBJ & .782 & .758 & .756 & .756 & .697 & .684 & .679 & .607 & .500 \\
\hline
\end{tabular}

Note - The row and column elements are ordered according to the maximum likelihood strong stochastic ranking.

estimated in a series of 1000 Monte Carlo simulations. The relevant nonparametrically estimated percentiles in tests with approximate size $.01, .05, .10$ were $3.49,1.83$, and .81 respectively.

Except for the data for subject 3, the proper values of the test statistic are lower than those obtained by Tversky, and the maximum likelihood weak stochastic rankings are not isotonic with the expected values. Depending on the size of the test, the statistical decision made would differ depending on whether Tversky's test was used or ours. Tversky rejects weak stochastic transitivity in a test with size .05 for five subjects, whereas we would reject stochastic acyclicity for six.

\subsubsection{Strong stochastic transitivity}

In a paper published in 1971, Rumelhart and Greeno (1971) reported on the results of a series of choices from a set of nine alternatives made by 234 college subjects. The set of alternatives contained three political figures (L.B. Johnson $(L B J)$, Harold Wilson $(H W)$, Charles DeGaulle $(C d G)$, three athletes (Johnny Unitas $(J U)$, Carl Yastrzemski ( $C Y)$, A.J. Foyt $(A J F)$, and three movie stars (Brigitte Bardot $(B B)$, Elizabeth Taylor $(E T)$, Sophia Loren $(S L)$. Subjects were instructed to choose the person with whom they would rather spend an hour discussing a topic of their choosing. The choice proportions can be found in Table 4.5. 
This set of altematives was sampled by Rumelhart and Greeno with the intention to evaluate Restle's hypothesis on the influence of similarity between altematives in choice behavior. Restle (1961) had presented a probabilistic choice theory in which similarity between altematives was a key factor. In short, Restle's theory predicts that if two altematives share valued properties, a choice from them will not be affected by these shared properties.

Rumelhart and Greeno evaluated the applicability of Luce's (1959) and Restle's probabilistic choice theories. They defined a model specification of Restle's theory that is a generalization of Luce's model specification. Furthermore, Rumelhart and Greeno effectively demonstrated that any such model would be equivalent with a model of Thurstone's (1927) more general theory on probabilistic choice behavior.

Rumelhart and Greeno computed maximum likelihood estimates of the parameters of both Luce's model and of a constrained version of Greeno's (cf. Edgell, Geisler \& Zinnes, 1973). Based on the likelihood ratio test statistics, they demonstrated a bad fit for the former and a reasonable fit for the second model.

One difference between the probabilistic choice theories of Luce and Restle is that the former expects strong stochastic transitivity to hold in BCP structures, whereas Restle's only expects the moderate variety. This prediction can be evaluated with the strategy proposed in this chapter.

We calculated maximum likelihood estimates under moderate stochastic transitivity, which were equal to the choice proportions. The test statistic was equal to zero and the maximum likelihood moderate stochastic ranking was:

\section{$\begin{array}{lllllllll}\overrightarrow{C Y} & B B & J U & A J F & E T & S L & C d G & H W & L B J\end{array}$}

Maximum likelihood estimates under strong stochastic transitivity can be found in Table 4.6. The maximum likelihood ss ranking was

$\begin{array}{lllllllll}\overrightarrow{C Y} & B B & J U & A J F & C d G & E T & H W & S L & L B J\end{array}$

This ranking differs from the maximum likelihood MS ranking, and also from the ranking that is isotonic with the values of the model parameters estimated by Rumelhart and Greeno for both the Luce and the Restle model:

\begin{tabular}{lllllllll}
\hline$B B$ & $C Y$ & $J U$ & $A J F$ & $E T$ & $C d G$ & $S L$ & $H W$ & $L B J$
\end{tabular}


Table 4.7

Choice proportions, based on data reported by Greenberg (1965)

\begin{tabular}{cccccccccc}
\hline & A & B & C & D & E & F & G & $H$ & I \\
A & .500 & .141 & .196 & .178 & .307 & .331 & .380 & .417 & .509 \\
B & .859 & .500 & .202 & .270 & .374 & .423 & .411 & .497 & .571 \\
C & .804 & .798 & .500 & .337 & .423 & .479 & .534 & .564 & .626 \\
D & .822 & .730 & .663 & .500 & .485 & .540 & .583 & .613 & .755 \\
E & .693 & .626 & .577 & .515 & .500 & .650 & .650 & .773 & .791 \\
F & .669 & .577 & .521 & .460 & .350 & .500 & .736 & .761 & .859 \\
G & .620 & .589 & .466 & .417 & .350 & .264 & .500 & .785 & .847 \\
H & .583 & .503 & .436 & .387 & .227 & .239 & .215 & .500 & .847 \\
I & .491 & .429 & .374 & .245 & .209 & .141 & .153 & .153 & .500 \\
\hline
\end{tabular}

Note. The row and column elements are ordered according to the maximum likelihood unfolded order.

The value of the test statistic we obtained was 20.36 . The boundaries of the critical regions for $\alpha=.01, .05, .10, .25$ estimated in a series of 1000 structures were $17.71,11.87,9.75$, and 7.11 respectively. We would therefore reject strong stochastic transitivity in a test with size .10 .

\subsubsection{Characteristic monotonicity}

Greenberg (1965) has presented a unidimensional unfolding strategy for paired comparisons data, based on a monotonic relation between choice proportions and interaltemative midpoints. Greenberg's technique is not probabilistic and suffers from the same implementation problems as Coombs' unfolding technique for choice rankings. Elsewhere (Bossuyt \& Roskam, 1987a), we have developed a nonparametric, probabilistic unfolding strategy that can be regarded as the probabilistic counterpart of Greenberg's proposal.

The difficulties in implementing the strictly isotonic relation between choice proportions and midpoints emerged rather unfortunately in the data Greenberg collected for the purpose of illustration. Greenberg asked 163 housewifes to choose out of all pairs from nine phrases. Each phrase described a possible attitude to the Volkswagen automobile. The content of the phrases ranged from excellent (A), over indifferent (E), to terrible (I). Greenberg's attempt to find a ranking of the midpoints isotonic with the choice proportions was not completely successful. 


\section{Table 4.8}

Constrained maximum likelihood estimates of choice probabilities for data reported by Greenberg (1965).

\begin{tabular}{cccccccccc}
\hline & A & B & C & D & E & F & G & H & I \\
A & .500 & .141 & .187 & .187 & .307 & .331 & .380 & .417 & .509 \\
B & .859 & .500 & .202 & .270 & .374 & .417 & .417 & .497 & .571 \\
C & .813 & .798 & .500 & .337 & .423 & .479 & .534 & .564 & .626 \\
D & .813 & .730 & .663 & .500 & .485 & .540 & .583 & .613 & .755 \\
E & .693 & .626 & .577 & .515 & .500 & .650 & .650 & .767 & .791 \\
F & .669 & .583 & .521 & .460 & .350 & .500 & .736 & .767 & .851 \\
G & .620 & .583 & .466 & .417 & .350 & .264 & .500 & .785 & .851 \\
H & .583 & .503 & .436 & .387 & .233 & .233 & .215 & .500 & .851 \\
I & .491 & .429 & .374 & .245 & .209 & .149 & .149 & .149 & .500 \\
\hline
\end{tabular}

Note - The row and column elements are ordered according to the maximum likelihood unfolded order.

Our probabilistic technique based on Greenberg's relies on one property of probabilistic choice behavior called midpoint monotonicity. The restrictions that define this condition are a superset of the inequalities that define characteristic monotonicity. We evaluated the latter condition using our evaluation strategy. We first reconstructed a structure of frequencies by multiplying the choice proportions reported in Greenberg (1965) by 163 , and then recalculated the choice proportions. These can be found in Table 4.7.

Maximum likelihood estimates under characteristic monotonicity are listed in Table 4.8, with the row and column indices in the maximum likelihood unfolded order. Not surprisingly, this ranking corresponds to the a priori ranking of the nine statements.

The value of $-2 \log$ likelihood ratio test statistic we obtained was .43 . We generated a series of $10009 \times 9$ matrices with binomial deviates from binary choice probability structures in which characteristic monotonicity was satisfied, with $n=163$. The boundaries of the critical regions for $\alpha=.01, .05, .10, .25$ estimated in this series of 1000 structures were 16.13, 12.53, 10.15, and 7.58 respectively. In a test with an approximate size of $\alpha=.05$, characteristic monotonicity could not be rejected. A midpoint unfolding model may show a reasonable fit to this model of data. 



\section{An experimental evaluation of probabilistic unfolding theories}

In this chapter we report on two experiments that were designed for a comparative evaluation of the applicability of existing theories on probabilistic unfolding, and in which the evaluation strategy unfolded in Chapter 3 and Chapter 4 was used. Each experiment consisted of a collection of paired comparisons tasks.

\subsection{EXPERIMENT 1}

Some thirty years ago, Coombs (1958) published results on an experimental evaluation of his theory on probabilistic unfolding. The choice task he created for this purpose has become known as the "Amsterdam experiment". Coombs and his coworkers asked four subjects - two men and two women - to rank chips of varying shades of gray according to their idea of prototypical gray.

Coombs had developed a probabilistic unfolding theory with a unidimensional psychological unfolding space. Any potential multidimensionality of the psychological space would have seriously crippled the ability to draw sound conclusions from an experiment. A set of shades of gray therefore seemed to form a suitable collection of stimuli, since increasing luminance is likely to yield a unidimensional space.

The four subjects who participated in Coombs' Amsterdam experiment were each asked to rank subsets of four stimuli from the collection of shades of gray. Based upon the subjects' rankings, Coombs calculated four structures of binary choice frequencies. After examining the number of violations of strong stochastic transitivity in the structures of choice proportions, Coombs concluded that his random coordinate assumptions were well supported.

Coombs' experiment has become something of a standard; the Amsterdam experiment has become one of the experiments most frequently referred to in the literature on probabilistic choice behavior. For this reason, a replication of his task with paired comparisons seemed a right start for our evaluation strategy. By asking subjects similar questions, and applying our evaluation strategy, we could compare our conclusions with Coombs', at the same time creating an opportunity to evaluate all theories on probabilistic unfolding that have been 
proposed after Coombs' initial approach. Furthermore, like Coombs' theory, many if not most of the more recent theories are restricted to unidimensional applications.

To eliminate the inconveniences inherent to repeated presentations, we preferred to estimate choice probabilities in a group of subjects. One of the tasks had to be a replication of Coombs' procedure. Yet in such a task we would never be able to test properties involving the ideal point, since we see no way to determine with sufficient certainty the location of each individual's ideal. Deciding on the location of a "group" ideal would be an even harder task. It is not self-evident to use the model of the data to decide which alternative was the ideal. The first element in the maximum likelihood weak stochastic ranking is not necessarily the ideal, or not even the element closest to the ideal.

To test the ideal point conditions, we therefore decided to include two tasks of paired comparisons with a reference stimulus. Each reference stimulus would be one of the shades of gray used. Within each task, we could evaluate the ideal point conditions IP.1 to IP.3. With the system of choice frequencies from both tasks, condition IP. 4 could be put on test as well, as the symmetry condition and, upon a proper selection of the reference stimuli, the unilaterally decreasing/increasing condition.

\subsubsection{Method}

Subjects

Subjects were recruited by placing notices in the departments of psychology and sociology of the Catholic University of Nijmegen. Each notice contained the announcement that the experiment would involve an evaluation of the perception of shades of gray, and that subjects would receive 8 guilders for their participation; 43 subjects participated.

\section{Stimuli}

By exposing photographic film for different periods we obtained sets of sheets with luminance values $.5, .7, \ldots .2 .3$. When projected against a screen, these sheets produced ten shades of gray, which will be referred to as stimuli $g_{1}, \ldots, g_{10}$ respectively. In three sets of 45 slides each, a piece of carbon paper was framed. Each piece had two $5 \mathrm{~mm}$ circularperforations, separated by $3 \mathrm{~mm}$, horizontally aligned and centered. By placing pieces of the exposed photographic film behind these perforations, we obtained three sets of all 45 pairs of the ten sheets.

Each set of slides was arranged in a tray. In each tray, the pairs of stimuli were arranged in a distinct spaced order, which is a presentation order for completely balanced designs that maintains the greatest possible separation 
between pairs having a stimulus in common (Ross, 1934). We made two additional slides with one perforation only, and pieces of exposed film with luminance values 1.1 and 1.3 respectively. These slides would act as reference stimuli. The shades of gray produced by these slides were identical to the stimuli $g_{4}$ and $g_{5}$ respectively.

\section{Procedure}

Subjects were run individually. They were placed behind a table at 2.5 meter from a projection screen, and had to perform at least three tasks. Each task consisted of 45 sequences, preceded by 10 trial sequences.

In two tasks subjects had to make paired comparisons with a reference stimulus. Each sequence in this task started with a 5 sec presentation of a single shade of gray, the reference stimulus, followed by a $1.5 \mathrm{sec}$ presentation of a pair of stimuli. After this pair had disappeared, a new sequence was started, with the same reference stimulus but followed by the next pair in the spaced order. Subjects were instructed to choose from each pair the stimulus most similar in grayness to the reference stimulus. In one triadic comparisons task (TR4), we used stimulus $g_{4}$ as the reference stimulus, in the other (TR5) stimulus $g_{5}$ was used. After the 45 th sequence the reference stimulus was displayed once more.

In the simple paired comparisons task (STG) each sequence started with a $5 \mathrm{sec}$ interval in which nothing was projected, and a $2 \mathrm{sec}$ presentation of a pair of stimuli. Subjects were instructed to choose from each pair the stimulus that resembled best what they personally considered to be prototypical gray.

Subjects expressed their choices after the presentation of a pair of stimuli by pressing one of two keys, which were connected to a small thermal printing unit. Subjects were instructed to make a choice, even when in doubt. Each subject performed two tasks once, and one task twice. The experiment started with the task that had to be repeated, and ended with the second presentation of this task. For each subject, the three spaced orders were randomly assigned to the three tasks. For the task to be repeated, one of the three spaced orders was selected at random, and reversed. Approximately 40 minutes were needed for each run of four tasks, instructions included.

\subsubsection{Results}

Since 43 subjects participated and several of them made two tasks, we finally obtained two times 59 individual structures of choices for tasks TR4 and TR5 respectively, and 67 structures of choices for the simple paired comparisons task STG. For each task, structures of binary choice frequencies were computed. 
The corresponding choice proportions can be found in Appendix B. The structures were used to evaluate properties of probabilistic choice behavior with the evaluation strategy discussed earlier.

For all tests, an approximate size of .10 was a priori selected. Whenever the distribution of the test statistic under the null hypothesis could not be computed nor estimated, a series of 250 Monte Carlo simulations was used to produce a nonparametric estimate of the relevant quantile(s), using order statistics. Statistics of these Monte Carlo series can be found in Appendix C. For condition IP.4, the unilaterally decreasing/increasing and the bilateral condition, series of 1000 simulations were used. For the symmetry condition, equation [4.35] was used to compute the test criterion. Test statistics and decisions based on them are summarized in Table 5.1.

\section{(1) Stochastic transitivity}

For each task one maximum likelihood weak stochastic ranking was found. All three rankings are perfectly consistent with the a priori luminosity ranking. This means that the weak stochastic rankings can be regarded as "folded" luminosity rankings, with the "fold" at the reference stimulus in TR4 and TR5, and at stimulus $g_{8}$ in STG.

For all three tasks, stochastic acyclicity was satisfied in the structure of choice proportions. This means that maximum likelihood estimates of the choice probabilities were equal to the choice proportions, and the values of the test statistic for stochastic acyclicity and weak stochastic transitivity were zero.

The moderate stochastic rankings were nearly identical to the weak stochastic rankings. Yet moderate stochastic transitivity had to be rejected in TR4. Strong stochastic transitivity had to be rejected in all three tasks.

Ideal point

As we explained in the introduction, the ideal point conditions could not be evaluated in the paired comparisons task STG. We computed maximum likelihood weak stochastic rankings for each subject separately, based on the choices made in task STG. This resulted in a heterogeneous collection of rankings, which supported our a priori belief on the low likelihood of a unanimous choice on what shade of gray resembled best "true gray".

We evaluated the ideal point condition in TR4 and TR5. Condition IP. 1 could be rejected in neither of the tasks. Maximum likelihood estimates of the choice probabilities were equal to the choice proportions, and the test statistics were zero. Condition IP.2 had to be rejected. Discrimination was not necessarily sharpest when one of the altematives was identical to the reference stimulus. Condition IP. 3 had to be rejected as well. The reference stimulus was not always correctly identified when presented. 


\section{Table 5.1}

Test statistics and decisions in Experiment 1.

Test Statistics

\begin{tabular}{lccccccccc} 
Task & STA & MS & SST & IP.1 & IP.2 & IP.3 & CM & BM & BCM \\
\hline TR4 & .00 & $12.75^{*}$ & $196.03^{*}$ & .00 & $58.71^{*}$ & rej. & $38.08^{*}$ & $45.19^{*}$ & $24.34^{*}$ \\
TR5 & .00 & 3.51 & $113.38^{*}$ & .00 & $7.20^{*}$ & rej. & 16.22 & $14.59^{*}$ & 9.19 \\
STG & .00 & 9.25 & $65.83^{*}$ & n.a. & n.a. & n.a. & 15.42 & $26.43^{*}$ & $14.37^{*}$
\end{tabular}

Note - An asterisk denotes that the property had to be rejected in the corresponding structure, using a test with an approximate size of .10; rej.: rejected; n.a.: property not applicable.

Condition IP. 4 could be tested with the choice frequencies in TR4 and TR5 for all pairs $\left\{g_{4}, g_{j}\right\}$ and $\left\{g_{5}, g_{j}\right\}$ with $j=1,2, \ldots, 10$ (excluding $\left\{g_{5}, g_{s}\right\}$ and $\left\{g_{4}, g_{4}\right\}$ ), involving 17 pairs of probabilities. This condition had to be rejected (Test statistic: 47.48; Test criterion: 1.98). Discrimination in a pair of stimuli was not necessarily easier when one of the altematives was physically identical to the reference stimulus.

The symmetry condition could be tested with the pair $\left\{g_{4}, g_{5}\right\}$ and the choice frequencies for TR4 and TR5. the choice proportions were .576 for $\left\{g_{4}, g_{5}\right\}$ in TR4 and .746 for $\left\{g_{5}, g_{4}\right\}$ in TR5. The hypothesis of equality had to be rejected. The value of $-2 \log$ likelihood ratio test statistic was 3.81 . The test criterion in a test with size .10 , computed using the probabilities computed through equation [4.35] was 2.81 .

The bilateral condition could be tested with the choice frequencies for all pairs $\left\{g_{i}, g_{j}\right\}(i=1,2,3, j=6,7, . ., 10)$ in TR4 and TR5. This condition could not be rejected (Test statistic: .33; Test criterion: 1.85).

The unilaterally decreasing/increasing condition was tested using the choice frequencies for all pairs in the subsets $\left\{g_{1}, g_{2}, g_{\}}\right\}$and all pairs in $\left\{g_{6}, g_{7}, g_{8}, g_{9}, g_{10}\right\}$, in TR4 and TR5. We looked for the maximum likelihood estimates of the corresponding choice probabilities in the subdomains of the parameter space satisfying the unilaterally decreasing/increasing condition respectively.

The generalized likelihood ratio test statistics were 9.51 and 10.36 for the increasing and decreasing condition respectively, which made the data look more likely under a set of probabilities satisfying the unilaterally increasing condition. The estimated .90 quantile of the distribution of the statistic under each condition was 1.44 , so none of the two conditions could be rejected. 
In all three tasks, the maximum likelihood unfolded orders under characteristic monotonicity were identical to the underlying luminosity rankings, except for an inversion of stimuli $\left(g_{5}, g_{4}\right)$ in TR4 and $\left(g_{5}, g_{6}\right)$ in TR5. Characteristic monotonicity had to be rejected in TR4 however.

The maximum likelihood unfolded orders under bilateral monotonicity were identical to the underlying luminosity rankings in tasks TR4 and TR5, except for the same inversion of adjacent stimuli $\left(g_{5}, g_{4}\right)$ in TR4. The maximum likelihood order in STG deviated severely from the luminosity ranking. Bilateral monotonicity had to be rejected in all three tasks.

The inversion of stimuli $\left(g_{5}, g_{4}\right)$ reappeared in the maximum likelihood unfolded order under bilateral/characteristic monotonicity in TR5. Both in TR4 and STG, the solution ranking equalled the luminosity ranking, yet bilate$\mathrm{ral} /$ characteristic monotonicity had to be rejected.

\subsubsection{Discussion}

If Coombs' assumptions for this kind of choices had been valid, we would expect all properties necessarily implied by the random coordinate theory (with single sampling of the ideal coordinate) to hold. These are

(1) no violations of moderate stochastic transitivity, but very likely violations of strong stochastic transitivity,

(2) no violation of the ideal point conditions IP.1, but very likely violations of IP.2, IP.3 and IP.4,

(3) no violation of the symmetry condition, no violation of the bilateral condition

(4) the unilaterally increasing condition,

(5) either bilateral/characteristic or simple characteristic monotonicity, and a recovery of the physical luminosity ranking in the search for the maximum likelihood unfolded order under these conditions.

We feel that these predictions were rather well supported.

(1) The first set of predictions was well supported in tasks TR5 and STG. Though a majority of probabilistic unfolding theories expects at least strict stochastic transitivity in probabilistic choice behavior, even strong stochastic transitivity seemed to be clearly violated in both sets of paired comparisons. Despite the similarity between tasks TR4 and TR5, moderate stochastic transitivity seemed plausible in the second, but not in the first. 
(2) With respect to the ideal point conditions, there were no violations of IP.1. In TR4 as well as in TR5, a majority of the subjects correctly identified the reference stimulus when it was presented. There was less support for the other ideal point conditions. As could be expected from results in similar experiments with nontrivial comparisons (for an example, see Krantz, 1967), the reference stimulus was not always correctly identified, so IP.3 had to be rejected. Similarly, though conditions IP.2 to IP.4 seem to have an intuitive appeal, they were unlikely in the comparisons we observed.

(3) It is not entirely clear how the rejection of the symmetry condition in TR4-TR5 has to be interpreted. This rejection may indicate that the metric of the psychological spaces in the tasks TR4 and TR5 differed, contrary to what we intended to achieve. In both tasks we used similar stimuli, only the reference stimulus was different, and the same subjects made judgments in TR4 and TR5. Perhaps there is a substantial functional difference between a similarity comparison of $\left\{g_{4}, g_{3}\right\}$ with $g_{4}$ and a comparison of $\left\{g_{4}, g_{3}\right\}$ with $g_{5}$, which is not reflected in the functional identity of two pairs of distances. On the other hand, if the space had been really dissimilar, we would have expected a rejection of the bilateral condition.

(4) If the subjective metric was not the same in both tasks, the test of the unilaterally decreasing/increasing condition is either biased or of little power. The data were more plausible under the increasing condition, yet both the increasing condition and the decreasing condition had to be rejected.

(5) Rather surprisingly, we had to reject all three unfolded order conditions in TR4, which contrasts with the rejection of bilateral monotonicity only in TR5, despite the fact that for both tasks the underlying luminosity ranking reappeared in all solutions. In the simple paired comparisons task STG, we could not reject characteristic monotonicity, but had to reject bilate$\mathrm{ral} / \mathrm{characteristic}$ monotonicity. Since the restrictions of $\mathrm{CM}$ are a subset of those of BCM, these results are hard to explain.

Concluding, we may say that within TR5, the predictions from Coombs' theory could not refuted, despite a lack of support in TR4, and the set of the comparisons with a reference stimulus taken as a whole. At the same time these results lead to the related conclusion that almost all competing theories seemed ill suited for data collected in these tasks. Strong stochastic transitivity was very unlikely, and this rules out all strong unfolding models, random distance models and the double sampling models of the Coombs-Zinnes-Griggs random coordinate theory. 
The only plausible models left are models of the Coombs-Zinnes-Griggs theory (single sampling - Case II), possibly one with the coordinate of the ideal as the single random variable (Case III), or (functionally equivalent) midpoint unfolding models. We are tempted to conclude that a Case II random coordinate model seems more acceptable in task TR5, whereas a Case III random coordinate model (or midpoint unfolding model) seems more suited for the data of STG. We find some support for this preference in the test statistics, but we can also explain it in terms of sizes of variances in the corresponding models.

In the comparisons with a reference stimulus both the ideal and the altematives were physically presented to the subjects, and in much the same way. Since there hardly could have been any additional intersubjective variability in the location of the ideal and the alternatives, the variances of the corresponding distributions were probably approximately equal. Intersubjective variability was, however, very likely to appear in the simple paired comparisons task, since in this task the ideal - each subject's conception on prototypical gray - had to be subjective. This subjectivity was supported by the wide assortment of wS rankings that emanated from the analysis of the individual choice structures. Due to a fusion of intrasubjective and intersubjective variability, the overall variance of the ideal coordinate distribution was very likely to exceed the variances of the distributions for the alternatives. With an increasing ratio of variances, one ultimately moves from a Case II to a Case III random coordinate model.

\subsection{EXPERIMENT 2}

In designing Experiment 2 we had two goals in mind. We wanted to gain more experience with our evaluation strategy, and we hoped to be able to elicit choice behavior for which a distinct class of theories might tum out to be appropriate.

First we designed a task, in which subjects were asked through paired comparisons what they thought to be the maximum penalty in the Criminal Code for a number of well-defined crimes. As in Experiment 1, we wanted to minimize the confounding impact of a multidimensional psychological space. A set of altemative imprisonment terms of increasing duration is likely to be located unequivocally on a unidimensional continuum. Furthermore, the unambiguous nature of the imprisonment terms may minimize the uncertainty regarding their location in the psychological space, which is one potential source of choice variability.

In a second choice task we wanted to ask subjects for their preferences in a set of eleven political parties of the Netherlands. Political preferences have always been a favorite territory of probabilistic choice modelers (e.g. Van 
Schuur, 1984), which explains this choice. We also wanted to ask subjects what party they intended to vote for if elections were to be held the other day. Though an answer to this question may not necessarily reveal a subject's ideal party in terms of its location on the left-right spectrum, it may serve as an extemal indication. By aggregating choices for subjects with identical vote intentions, we would be able to create a choice frequency system in which the ideal point conditions could be tested.

Next to its popularity as a field for unfolding applications, we had a second consideration in mind when incorporating preferences for political parties in this experiment. It seemed not implausible to us that we would find support for the unilaterally decreasing condition in a model of data generated in such a task. We suspected subjects with similar vote intentions to have less articulate preferences for parties located further away from their favorite area of the political left-right spectrum, and more pronounced, or more homogeneous opinions for parties close to this preferred region.

The third choice task grew out of a series of discussions on the applicability and interpretability of random coordinate theories in Experiment 1. If the choice variability is attributed to a random variability in the location of the alternatives and the ideal, was it then due to physical processes only, or (also) related to the way we defined the tasks with paired comparisons with a reference stimulus? More specifically, we wondered whether we could create a choice situation that would lead to conclusions similar to the ones made in the first experiment, even if we increased the identifiability of stimuli. This led us to a set of tasks involving comparisons with a reference stimulus, functionally similar to tasks TR4 and TR5 of Experiment 1, but with two-digit numbers replacing shades of gray.

\subsubsection{Method}

\section{Subjects}

Subjects were recruited by placing notices in all departments of the Catholic University of Nijmegen and in the local University newspaper. Each notice contained the announcement that choice behavior was the subject of the experiment, and that participants would receive 8 guilders for their cooperation. Within three weeks 85 subjects volunteered and participated.

Stimuli

In a first set of tasks the altematives were seven different imprisonment terms: $2,4,6,9,12,15$, and 18 years. The reference stimuli were what each subject thought to be the maximum penalty for the following six crimes: petty extortion 
$\left(c_{1}\right)$, counterfeiting $\left(c_{2}\right)$, intentional endangering of public transport or traffic $\left(c_{3}\right)$, robbery $\left(c_{4}\right)$, bribery of a judicial officer $\left(c_{5}\right)$, and rape $\left(c_{6}\right)$. The actual maximum penalty in the Criminal Code of the Netherlands is 9 years of imprisonment for $c_{1}$ to $c_{9}$, and 12 years for $c_{6}$.

In each task one crime was the reference stimulus. Each task $\mathrm{CCi}$ was labelled by the crime $c_{i}$ used in it. Task CC4, for example, had $c_{4}$, robbery, as the reference stimulus.

In a second set of tasks the altematives were eleven political parties of the Netherlands, presented by their acronyms: CDA (christian-democrats), CPN (communists), D66 (left-wing liberals), EVP (left-wing Christians), PVDA (socialists), PPR (socialist ecologists), PSP (pacifist socialists), VVD (right-wing liberals), RPF, SGP, GPV, (small right-wing Christian parties).

In a third set of tasks the alternatives were ten two digit numbers: $08,14,27$, $31,46,59,62,70,85$ and 93 . The selection criteria had been the following:

(1) the absolute difference of the two digits in a number exceeds 2 ,

(2) no two numbers are composed of the same two digits,

(3) all numbers can be ranked without ties according to their absolute difference with number at a central position in the simple magnitude ordering.

In the present set, the latter two rankings are: $46,59,31,62,27,70,14,08,85$, 93 for stimulus 46 , and $59,62,70,46,85,31,27,93,14,08$ for stimulus 59 .

\section{Procedure}

Subjects were run individually, and had to perform all ten tasks. They were seated in front of a monitor with a black and white screen, on which the instructions and the altematives for each task were displayed. To read a new screen of instructions, to start a new task or to make a choice, they pressed one of two keys (left or right).

(1) The instructions for the first set of tasks included a general introduction on the principals of the Criminal Code, and the implicit seriousness ranking in the maximum penalties in the Criminal Code. Then subjects received the Criminal Code description of each of the six crimes, from which, for obvious reasons, the maximum penalty was omitted.

The set of all pairs of seven imprisonment terms led to six series of 21 presentations, one series for each crime. Each presentation started by a 1200 msec clearing of the screen. The label of a crime (say, "robbery") and two numbers expressing years of imprisonment were presented next. Subjects were instructed to choose the number which came closest to what they expected to be the maximum penalty for the crime displayed. Each 
presentation was followed by the next one $300 \mathrm{msec}$ after the subject had made a choice.

The presentations for all six tasks were intertwined into a single series of 126 presentations, preceded by 6 trial presentations. These 126 presentations were organized as follows. For each crime the 21 pairs of imprisonment terms were arranged in a distinct spaced order. The first 6 of the 126 presentations included the first pair in each of these 6 orders, in a random order. The 7 th to the 12th presentation contained the second pair in each spaced order, again randomly ordered, and in such a way that the 7 th presentation did not have the same reference stimulus as the 6th. Continuing this way we obtained an intertwined presentation order for the 6 sets of 21 presentations.

(2) In the first task (PP1) of the second set of tasks (political parties) simple paired comparisons had to be made. Subjects were instructed to choose the political party they sympathized with, or, if they felt a preference for neither, the party they resented less. All 55 pairs of the 11 altematives were presented in a series of 55 presentations, preceded by 6 trial presentations. As in all other tasks, these 6 trial presentations were randomly sampled from the actual series. Each presentation started with a $1200 \mathrm{msec}$ blanking of the screen, followed by a presentation of a pair of political party acronyms. This presentation was followed by the next, $300 \mathrm{msec}$ after the subject had made a choice. The 55 presentations contained all pairs of political parties in a spaced order. For each subject, a spaced order was randomly selected.

In the second task (PP2) of this set subjects were asked to pick the party they would vote for if elections were to be held the other day. A list of the 11 parties, names and acronyms, used in the first task PP1 was displayed on the screen, in a sequencing randomly reselected for each subject. Two options were added to this list: "would vote for a party not on this list" and "would not vote". Subjects expressed their vote intention by using a key to move a pointer through the list until the desired option was indicated. The second key could be used to confirm the selection made.

(3) The third set of tasks was based on comparisons with a reference stimulus, involving simple arithmetic. Three numbers (the reference stimulus and two altematives) were displayed, and subjects were asked to pick the alternative for which the absolute difference with the reference stimulus was smaller. Due to the selection of the alternatives, an arithmetically correct choice could always be made. 
In one task, 46 was the reference stimulus, in the other 59. and the tasks were labelled by the reference stimulus used in it: N46 and N59. Each task consisted of 45 presentations, preceded by 6 trial presentations. Each presentation started by a $5 \mathrm{sec}$ presentation of the reference stimulus, followed by a 250 msec presentation of a pair of numbers. The 45 presentations contained all 45 pairs of the stimuli in a spaced order.

Each run through all tasks started with the instructions for the first set (CC1-CC6), followed by the 6 trial presentations and presentations 1 to 42 of this set. Next the subject received the instructions for the first task of the third set (either N46 or N59, selected at random), 6 trial presentations and all 45 presentations of this task. Next the instructions for task PP1 were displayed, followed by 6 trial presentations and the first 27 presentations of this task. This was followed by: presentations 43 to 84 of the "crime" tasks, instructions and 45 presentations of the remaining "numbers" task, presentations 28 to 55 of the "political parties" task PP1, presentations 85 to 126 of the "crimes" set, and task PP2.

All tasks were monitored by a minicomputer. All random selections, including the random assignment to the spaced orders, were made for each subject individually, by the custom made presentation package running on this mini. On average, subjects took app. 50 minutes to complete all tasks.

\subsubsection{Results}

For the tasks $\mathrm{CC} 1$ to $\mathrm{CC} 6, \mathrm{~N} 46, \mathrm{~N} 59$ and PP1 the 85 sets of choices were used to compute nine sets of binary choice frequencies. This way three systems of choice frequencies were obtained, one for each set of tasks. Furthermore, the answers in task PP2 were used to distribute the sets of choices for PP1 over nine subsets, collecting the choices of subjects with identical vote intentions. This way a fourth system, with nine sets of choice frequencies, could be created. The latter system was only used to evaluate the ideal point conditions. Structures of choice proportions can be found in Appendix B.

All tests used had an approximate size of .10. Whenever the distribution of the test statistic was unknown, a series of 250 Monte Carlo simulations was used to estimate relevant quantiles of the distribution nonparametrically, using order statistics. Statistics of these Monte Carlo series can be found in Appendix C. For condition IP.4, the unilaterally decreasing/increasing and the bilateral condition, series of 1000 simulations were used. Test statistics and decisions based on them are summarized in Table 5.2. 
The results on the probabilistic transitivity conditions were very similar in all tasks. All structures of binary choice proportions satisfied weak stochastic transitivity. Moderate stochastic transitivity could not be rejected, but strong stochastic transitivity was very unlikely. In the first set of tasks, all solutions under weak, moderate and strong stochastic transitivity were consistent with the underlying duration dimension. A majority of subjects expressed left-wing sympathies in task PP1, making the social-democrats (PVDA) the first party in the maximum likelihood weak stochastic ranking, with the right-wing Christian parties at the end positions.

In the third set of tasks, involving arithmetic subtractions, the solutions for the stochastic transitivity conditions did not differ much from one another both within N46 and N59. All solutions were consistent with the underlying magnitude ordering, yet they showed impressive deviations from the arithmetic differences. In the maximum likelihood weak stochastic ranking in N46, only the first two stimuli ended up in their correct position (i.e. based on the arithmetic differences): $46,59,62,31,70,27,14,85,08,93$. In N59 only the first two and the last three stimuli were positioned correctly: $59,62,46,70,31,27,85,93$, $14,08$.

\section{The ideal point}

The ideal point conditions IP. 1 to IP.4, and the symmetry condition, could not be tested in the first set of tasks, $\mathrm{CCl}$ to $\mathrm{CC} 6$. This was due to the impossibility of predicting whether or not a subject's opinion on the maximum penaity (the ideal in this situation) was one of the available altematives.

Upon an inspection of the maximum likelihood weak stochastic rankings, we decided to treat all pairs in the subset $\{9,12,15,18\}$ and the pair $\{2,4\}$ as located unilaterally to the common opinion on the severity of the maximum penalty. Next we looked for the permutation of the 6 crimes that maximized the likelihood under the bilateral condition [2.14] (which is supposed to hold for all known probabilistic unfolding theories) and either the unilaterally decreasing or increasing condition.

The maximum likelihood permutation tumed out to be the one satisfying the

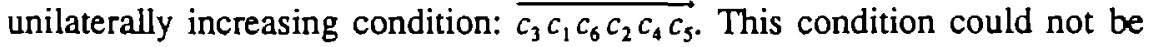
rejected (Test statistic: 1.65 ; Test criterion: 4.77 ).

As described earlier, the answers on the vote intention question in PP2 were used to redistribute the individual sets of choices in PP1, grouping subjects with similar vote intentions. In the sets of choice frequencies created this way the ideal point conditions were tested. Condition IP. 1 and IP. 2 could not be rejected. The values of the test statistic for both hypotheses were zero, except for the 


\section{Table 5.2}

Test statistics and decisions in Experiment 2.

Test Statistics

\begin{tabular}{lrrrrrrrrr} 
Task & STA & MS & SST & IP.1 & IP.2 & IP.3 & CM & BM & BCM \\
\hline CC1 & .00 & 1.04 & $88.80^{*}$ & n.a. & n.a. & n.a. & 1.46 & $38.42^{*}$ & 1.46 \\
CC2 & .00 & .00 & $64.75^{*}$ & n.a. & n.a. & n.a. & .00 & $26.99^{*}$ & .00 \\
CC3 & .00 & 1.15 & $63.23^{*}$ & n.a. & n.a. & n.a. & 3.95 & $20.91^{*}$ & 1.28 \\
CC4 & .00 & .07 & $66.14^{*}$ & n.a. & n.a. & n.a. & .35 & $23.48^{*}$ & .35 \\
CC5 & .00 & .00 & $43.64^{*}$ & n.a. & n.a. & n.a. & .00 & $19.70^{*}$ & .00 \\
CC6 & .00 & .36 & $81.30^{*}$ & n.a. & n.a. & n.a. & .40 & $38.55^{*}$ & .40 \\
PP & .00 & .00 & $61.10^{*}$ & n.a. & n.a. & n.a. & $174.21^{*}$ & 13.08 & 2.94 \\
N46 & .00 & .95 & $265.63^{*}$ & .00 & $109.28^{*}$ & rej. & 9.30 & $39.85^{*}$ & 6.45 \\
N59 & .00 & 3.95 & $273.68^{*}$ & .00 & $32.69^{*}$ & rej. & 16.98 & $39.21^{*}$ & 8.74 \\
\hline
\end{tabular}

Note- An asterisk denotes that the property had to be rejected in the corresponding structure, using a test with an approximate size of .10; rej. : rejected; n.a.: property not applicable.

data from the subjects who answered they intended to vote for the party VVD. In these data condition IP. 2 seemed to be violated. Condition IP. 3 was violated in all substructures, except in the sample corresponding to the party CDA. Only subjects who expressed a vote intention for the christian-democrats did choose their preferred party whenever it was presented in paired comparisons task PP1.

We also tested the unilateral condition, by estimating choice probabilities in the subsystem containing choice frequencies for all subjects with vote intentions for the parties CPN, PSP, PPR, PVDA or D66. The test statistic was based on all choice probabilities for the pairs out of the subset (CDA, VVD, EVP, GPV, SGP, RPF\}. Though the data slightly favored the increasing condition, both the decreasing and increasing condition had to be rejected.

In tasks $\mathrm{N} 46$ and $\mathrm{N} 59$, the reference stimulus was also an element of the set of altematives, so all ideal point conditions could be evaluated. In both tasks condition IP.1 could not be rejected; test statistics were zero. Condition IP.3 had to be rejected. The number of correct identifications of the reference stimulus was lower than the number of presentations when the ideal was presented together with one of the altematives $(27,31,59,62,70,93)$ in $\mathrm{N} 46$ or the alternatives $\{14,27,31,46,62,70\}$ in N59. For example, only $60 \%$ of the subjects correctly identified 46 when presented with 59 , and only $62 \%$ identified 59 when presented with 46. 
Condition IP. 2 had to be rejected as well. Discrimination in pairs containing the reference stimulus not only lacked perfection, it was not necessarily sharpest. In task $\mathrm{N} 46$ for example, only $68 \%$ of the subjects correctly chose the reference stimulus in the pair $\{46,62\}$, whereas $99 \%$ picked 59 out of $\{59,62\}$.

The ideal point condition IP.4 had to be rejected (Test statistic: .97; Test criterion: .74).

The test statistic for the unilaterally increasing/decreasing conditions were 1.98 and 1.59 respectively. Both conditions had to be rejected (Test criterion: .92).

The bilateral condition could be tested with the choice frequencies of N46 and N59 and the set of all pairs $\{x, y\}$ with $x \in\{08,14,27,31\}$ and $y \in\{62,70,85,93\}$. This condition could not be rejected (Test statistic: .00; Test criterion: 1.36). The symmetry condition could not be rejected either. The value of $-2 \log$ likelihood ratio test statistic was .049 . The test criterion in a test with size .10 , computed using the probabilities computed through equation [4.35] was 2.782 .

The unfolded order

In each task of the first set, $\mathrm{CC} 1$ to $\mathrm{CC}$, the maximum likelihood unfolded order under bilateral/characteristic and characteristic monotonicity corresponded to the underlying severity ranking of the imprisonment terms. Both conditions could not be rejected. Bilateral monotonicity had to be rejected in all tasks. Except for $\mathrm{CC} 1$ and $\mathrm{CC}$, all solutions under bilateral monotonicity were identical to the underlying duration ranking.

In the political parties task PP1 we had to reject characteristic monotonicity. Bilateral nor bilateral/characteristic monotonicity could be rejected. Unlike the solution under bilateral monotonicity, the maximum likelihood unfolded order under bilateral/characteristic monotonicity parallelled a left wing - right wing ordering of the eleven political parties:

\section{EVP CPN PPR PSP PVDA D66 CDA VVD RPF GPV SGP.}

The only anomaly is the extreme left position of the (moderate) left-wing christian party EVP. In the unfolded order that resulted in the next-to-highest value of the likelihood, the parties were arranged identically to the maximum likelihood unfolded order, except for the EVP party, which ended up between the liberals VVD and the right-wing christian parties.

For the arithmetical tasks, N46 and N59, a single permutation emerged as the maximum likelihood solution both under characteristic, bilateral, and bilate$\mathrm{ral} / \mathrm{characteristic} \mathrm{monotonicity.} \mathrm{This} \mathrm{permutation} \mathrm{corresponded} \mathrm{to} \mathrm{the} \mathrm{under-}$ lying magnitude ordering. We had to reject bilateral monotonicity. 


\subsubsection{Discussion}

\section{(1) Criminal Code}

The set of "crimes" tasks was included in the experiment in order to obtain a choice situation that would lend itself easily to unidimensional unfolding, with the attractive feature that the number of tasks would allow a more refined test of the unilateral condition. Furthermore we hypothesized that all variability in the choices had to be attributed to an intra- and intersubjective variability in the location of the "ideal" (the subject's guess at the maximum penalty) on the underlying psychological continuum, due to the unambiguous presentation of the altematives (imprisonment terms of varying length). Therefore a random ideal coordinate model, or a model functionally similar in properties, as a midpoint model, might well be suited for the data.

This hypothesis was well supported. In every way the tests indicated the appropriateness of a unidimensional random coordinate model, single sampling of the ideal coordinate, or a data-equivalent midpoint model, rejecting the majority of the competing theories. The highest level of stochastic transitivity fulfilled (moderate) corresponded exactly to the predictions. All models of theories presupposing stronger transitivity conditions had to be ruled out. To the latter category belong all random distance models, and all strong unfolding models.

In all six substructures characteristic monotonicity could not be rejected. Deviations from characteristic monotonicity in the choice proportions were either small or absent, and the maximum likelihood unfolded orders under this condition were equal to the underlying magnitude ordering.

A remarkable result was produced by the procedure used to evaluate the unilateral condition. After a partition of the set of pairs into bilateral and unilateral pairs, the permutation maximizing the likelihood under either the decreasing or increasing condition tumed out to satisfy the latter. The unilaterally increasing condition is required in a random ideal coordinate model, single sampling.

The solution permutation under the unilateral condition turned out to be almost consistent with the unfolded order of these crimes, based on the maximum likelihood weak stochastic rankings in each task. Using Coombs' original terminology, the series of six maximum likelihood rankings forms a set of $I$ scales that can be unidimensionally unfolded. By ordering this set of ws rankings according to the maximum likelihood permutation under the increasing condition, each ranking can be derived from the ranking to its left or right by reversing adjacent altematives. 


$\begin{array}{rrrrrrrl}4 & 6 & 2 & 9 & 12 & 15 & 18 & (\mathrm{CC} 3) \\ 6 & 4 & 9 & 2 & 12 & 15 & 18 & (\mathrm{CC} 1) \\ 6 & 4 & 9 & 2 & 12 & 15 & 18 & (\mathrm{CC} 6) \\ 6 & 4 & 9 & 12 & 2 & 15 & 18 & (\mathrm{CC} 2) \\ 6 & 9 & 4 & 12 & 2 & 15 & 18 & (\mathrm{CC} 5) \\ 6 & 9 & 4 & 12 & 15 & 2 & 18 & (\mathrm{CC} 4)\end{array}$

In deterministic unidimensional unfolding, reversing adjacent altematives in sets of $I$ scales corresponds to crossing the corresponding interaltemative midpoint on the underlying psychological continuum.

\section{Political parties}

By including a paired comparisons task with political parties as stimuli we hoped to elicit a kind of probabilistic choice behavior that would fulfil a distinct pattem of properties. For example, we a priori considered it to be plausible that the unilateral decreasing condition would be satisfied, and the increasing condition violated, contrary to our predictions for the first experiment. We hypothesized that subjects with leftist sympathies would discriminate sharper between left-wing parties than between right-wing parties, and vice versa. This property of probabilistic choice behavior corresponds to the unilaterally decreasing condition. Because of their political sympathies, these subjects were assumed to be more aware of subtle ideological differences on the left side of the political spectrum, and comparable less aware of ideological idiosyncrasies of the small right-wing parties.

The test of this unilateral condition was handicapped because a majority of the participating subjects expressed a vote intention for one of the left-wing parties. Based on the test we used, both the increasing and decreasing condition had to be rejected.

For the ideal point conditions we expected fulfilment of the stronger conditions IP.2 and IP.3. Condition IP.2 was rather well supported, but condition IP. 3 was not satisfied. Except for the subjects with an expressed vote intention for the christian-democratic party (CDA) all participating subjects did not al ways choose the party they said they would vote for. Since the presentation of the parties was unambiguous, and the choices could be made without a time limit, the latter result is somewhat puzzling. A possible explanation could be that the choice frequencies were computed under the somewhat optimistic assumption that all subjects prepared to vote would have done so for their "ideal" party. This assumption could have been violated, since poll results indicated that a fraction of the electorate had made "strategic" vote decisions in the Parliament elections which were to be held shortly after the experiment. Maybe citizens do not always vote for the party they sympathize with most. Some vote hoping to support, or to break, a ruling coalition. 
The maximum likelihood unfolded order under bilateral/characteristic monotony mirrorred a widely accepted left-right ordering of the political parties, except for the strange position of the leftist Christians EVP. If we let ourselves be seduced to a posteriori reasoning, we might say that this position is due either to an the subjects' unfamiliarity with the party - which generally keeps a low profile - or to its inherent ambiguity of the party: a mixture of left-wing ideas based on Christian principles.

Our data-analytic procedure only looks for the maximum likelihood estimates, and the accompanying value of the test-statistic. A more general procedure might want to look at the five (or more) rankings that result in the highest values of the likelihood. Such an overview might give a better impression of the stability of the solution.

\section{Arithmetic}

In the tradition of Thurstone (1927), stimuli in probabilistic choice are thought of as giving rise to a "discriminal process" on a subjective continuum. This discriminal dispersion is usually invoked to explain inconsistencies in choice behavior. The shades of gray of Experiment 1 clearly fell into this category of stimuli. We may very well attribute the choice variability over and within subjects in tasks TR4 and TR5 to a variability in the location of the stimuli on the underlying subjective luminosity or "grayness" continuum.

By designing the arithmetical tasks N46 and N59 we wanted to create a situation similar to the one in tasks TR4 and TR5, except that all perceptual sources of ambiguity had been eliminated. Because stimuli were abstract numbers, the eventual ambiguity could only be attributed to the process of making the simple arithmetical calculations itself.

In general the estimated choice probabilities for tasks N46 and N59 were more extreme than the ones in the first experiment, as could be expected. There was less variability, and there were less mistakes. Yet the folded maximum likelihood weak stochastic rankings deviated severely from the true arithmetic differences.

To our surprise, the results of the tests were remarkably similar to the outcomes for tasks TR4 and TR5 of Experiment 1. Moderate stochastic transitivity could not be rejected, but all higher levels of transitivity had to be discarded. The data corresponded closely to a pattem satisfying bilateral/characteristic monotonicity, though characteristic monotonicity had to be rejected in task N59 only. Within the ideal point conditions, IP.1 was satisfied, but IP.2, IP.3 and IP.4 had to be rejected. 
Contrary to what was found in Experiment 1, the bilateral increasing condition and the symmetry condition could not be rejected for tasks N46 and N59. This seems to indicate that, unlike the tasks with the shades of gray, the two sets of arithmetic comparisons were more alike in terms of an common underlying psychological continua.

We wonder by which mechanisms patterns of choices in tasks as divergent as TR4-TR5 and N46-N59 were made so similar. One more puzzling result remains to be pointed at. From a sample of university students we would expect that they are able to compute quickly the arithmetical comparisons needed in the tasks. Yet the large amount of arithmetical blunders makes one wonder by which transformations subjects rendered the integers comparable.

\subsection{CONCLUSIONS}

The demands on a model of data that are put by the original, deterministic unfolding theory are rather austere; most sets of data fail to satisfy the conditions that are necessary for an unfolding representation. The attractiveness of the unfolding paradigm has stimulated choice modellers to look for modifications of the unfolding strategy that would enlarge the number of possible and interesting applications.

These numerous attempts can be localized in a no-man's land bordered by lines of trenches and ideological barricades. Discussions over barricades are seldom easy and the way in which they proceed tends to obscure the nature of the conflict itself. In our view, conflicting opinions on the value of less austere unfolding techniques can be traced back to diverging opinion on the presumed origins of these techniques.

From one line of trenches, an unfolding representation is looked upon as nothing more than an idealized structure with compeletely known properties. Given a set of choices, one can use a variety of techniques to find a representation that comes as close as possible to the ideal structure. From this "scaling" approach, any such technique is just a "black box" that can be studied: one can see if and how any alternative arives at a representation, and how reliable these solutions are (Heiser, 1981).

The trenches on the other side of the field are manned by supporters of what we might call the "theoretical" approach, without willing to accuse the opposite side of being entirely a-theoretical. There is a long-standing psychological tradition of linking representation techniques to theorizing on behavior. This tradition goes back to Fechner and Thurstone, and is exemplified in works from authors as Thurstone $(1927,1959)$, Luce (1959), Restle (1961) and Tversky 
(1972a, 1972b). For the scholars in this set of trenches, the value of a representational technique is closely related to the empirical plausibility of its theoretical assumptions and their corrolaries.

The numerous collection of proposals for a probabilistic unfolding theory follows the latter tradition, at least in majority. We feel that these origins should guide us in making a selection from this diversity of approaches. We could study them as "black boxes", evaluating global goodness-of-fit indexes, likelihoods and penalized likelihoods, but this would not do justice to their theoretical background. A closer look at the basic assumptions of the underlying theory, and their implications for probabilistic choice behavior may be more warranted. Furthermore, such a closer look can be totally independent from distributional assumptions and peculariaties in maximization procedures. Both are of minor or no relevance for a theory's basic validity, yet they can substantially influence the goodness-of-fit between a model of the data and a model of the theory.

Such an examination of necessary properties in probabilistic choice behavior is what we have attempted in this dissertation. To the necessary properties known from the literature on probabilistic choice, we added some others, more closely related to unfolding. Some of these properties were based on altemative probabilistic translations of Coombs' definition of the ideal alternative, others were related to a unidimensional unfolding space.

We did not claim that this approach is original. Yet a major limitation for any evaluation procedure based on these properties has been the absence of techniques to evaluate their validity in a statistically acceptable way. By formulating the properties as conditional ordinal restrictions in the estimation of choice probabilities, adding a branch search strategy, and using a generalized likelihood ratio test, we think we have succeeded in defining a legitimate statistical strategy.

The data-analytic strategy described in this dissertation can altematively be used as a data-analytic procedure of its own, to find the maximum likelihood preference ranking under a variety of probabilistic preference relations for example. or to find the maximum likelihood unfolded order under characteristic monotonicity. The algorithm presented for this purpose (Bossuyt \& Roskam, 1987a, 1989a) will be elaborated in the future. It is not all too difficult to adapt it for data from other choice tasks, such as pick $x$ out of $n$, or ranking tasks.

The same strategy can also be used to compare other probabilistic choice theories, unrelated to unfolding, or any other probabilistic modelling problem. Work is already in progress on a new variety of probabilistic unfolding theories, based on the features of feasible altematives rather than on global dissimilarity comparisons (Candel, 1989). 
We want to finish this set of reflections with a second view at the results of our experiments. From the assortment of theories on probabilistic unfolding that can be found in the literature after 1959, the year of Coombs' first proposal, only a minority seemed applicable to our models of data. This minority is functionally similar to Coombs' original random coordinate theory. Despite the fact that a majority of theories on probabilistic unfolding, both in and outside the unfolding paradigm, expect strong or even strict stochastic transitivity in probabilistic choice behavior, we found little evidence for this kind of consistency. This can be related to our experimental multiple-judgment design but, as such, it should serve as a warning to future choice modelers.

One probabilistic choice model - the random coordinate model with single sampling of the ideal coordinate - seemed an apt description of all structures of binary choice frequencies we collected in these two experiments. Depending on the task, the variance of the ideal coordinate distribution can largely exceed all other variances, ultimately making the model functionally equivalent to a midpoint unfolding model. The fact that this random coordinate model is a model of the theory of unfolding's founding father, Clyde Coombs, is a pleasant observation to end this dissertation. 



\section{References}

Ahrens, J.H., \& Dieter, U. (1974). Computer methods for sampling from gamma, beta, Poisson, and binomial distributions. Computing, 12, 223-246.

Ahrens, J.H., \& Dieter, U. (1980). Sampling from binomial and Poisson distrbutions: a method with bounded computation umes. Computing, 25, 193-208.

Akake, H. (1973). Information theory and an extension of the maximum likelihood pnnciple. In B. Petrov \& F. Csaka (Eds.), 2nd International symposium on information theory Budapest: Akademial Kıado.

Akarke, H. (1974) A new look at the statisucal model idenufication. IEEE Transactions on Automatlc Control, $A C-19,716-723$

Andnch, D. (1985). A probabilistic item response theory model for unfolding preference data Paper presented at the Amencan Educational Research Associauon Conference, Chucago, Illinoss.

Andrich, D. (1989). A probabilistic IRT model for unfoldıng preference data. Applied Psychological Measurement, 13, 193-216.

Aschenbrenner, M. (1981). Efficient sets, decision heunstics and single-peaked preferences. Journal of Mathematical Psychology, 23, 214-226.

Ayer, M., Brunk, H D., Ewing, G M , Reıd, W T., \& Silverman, E (1955). An empincal distribution for sampling with incomplete information. Annals of Mathematical Statistics, 26, 641-647.

Barlow, R.E., Bartholomew, D J., Bremner, J.M., \& Brunk, H.D. (1972) Statistical inference under order restrictions London: Wiley.

Beals, R., Krantz, D.M., \& Tversky, A. (1968). Foundations of muludimensional scalıng. Psychological Review, 75, 127-143.

Bechtel, G.G. (1968). Folded and unfolded scalıng of preferentual parr comparisons. Journal of Mathematical Psychology, 5, 333-357.

Bechtel, G.G. (1976). Multidimensional preference scalıng The Hague- Mouton.

Becker, G.M., DeGroot, M.H., \& Marschak, J. (1963). Probabilitues of choice among very sumilar objects. Behavioral Science, 8, 306-311.

Benneth J.F., \& Hays, W.L. (1960). Multıdımensional unfolding Determining the dimensionality of ranked preference data. Psychometrika, 25, 27-43.

Bezembinder, Th. (1981). Circulanty and consistency in paired comparisons. Brittsh Journal of Mathematical and Statistical Psychology, 34, 16-37.

Bezembinder, Th., \& Bossuyt, P.M.M. (1989). Strong stochastic transitivity in a multudimensional probabilisac unfolding model. Journal of Mathematical Psychology, 33, 496-499.

Black, D. (1948). On the rationale of group decision making. Journal of Political Economy, 56, 23-34.

Block, H.D., \& Marschak, J. (1960). Random ordenngs and stochasuc theones of response. In I. Olkın, S. Ghurye, W. Hoffding, W. Madow, \& H. Mann (Eds.), Contributions to Probability and Statistics Stanford: Stanford University Press. 
Bock, R.D., \& Jones, L.V. (1968). The measurement and prediction of judgment and choice San Francisco: Holden Day.

Bossuyt P.M.M. (1985). Preference functions in a strong unfoldıng model Internal Report 85 MA 02, Mathematical Psychology Group, K.U. Nijmegen.

Bossuyt, P.M.M., \& Roskam, E.E.Ch.I. (1985). A nonparametric test of probabilistic unfolding models Paper presented at the 4th European Meetung of the Psychometric Society, Cambndge, U.K.

Bossuyt P.M.M., \& Roskam, E.E.Ch.I. (1987a). Eindimensionale MaxumumLikelihood Entfaltung fur ein probabilssusches Modell ohne Annahmen uber die Vertellung. Zettschrift für Sozıalpsychologıe, 18, 282-294.

Bossuyt, P.M M., \& Roskam, E.E.Ch.I. (1987b). Testung probabilistuc choice models Communication \& Cognition, 20, 5-16.

Bossuyt, P M.M., \& Roskam, E E.Ch.I. (1989a). Maxımum likelıhood unidımensıonal unfoldıng in a probabilisuc model without parametnc assumptions. In H. Feger, K C. Clauer \& G. De Soete. New developments inpsychological choice modeling Advances in Psychology senes Amsterdam: Elsevier.

Bossuyt, P.M M., \& Roskam, E.E.Ch.I. (1989b). Testing probabilisuc choice models. In H. Feger, K.C. Clauer \& G. De Soete' New developments in psychological choice modeling Advances in Psychology senes Amsterdam: Elsevier.

Box, B.E.P., Hunter, W.G., \& Hunter, J.S. (1978). Statistics for experımenters. New York. Wiley.

Bradley, R.A. (1976). Science, statıstics and parred compansons Biometrics, 32 , 213-232.

Bulgren, W G. (1971). On representations of the doubly non-central $F$ distribution Journal of the American Statistical Association, 66, 184-186

Candel, M. (1989). The additive random feature model for unfolding an empincal test of critical predictions for linear trees Unpublished internal manuscrpt, Mathemancal Psychology Group, K.U. Nijmegen.

Carlson, B.W. (1984). Response mode and choice consistency: A test of unfolding theory. Journal of Mathematical Psychology, 28, 179-190.

Carroll, J.D. (1972). Individual differences and multudimensional scaling. In R.N. Shepard, A.K. Romney, \& S. Nerlove (Eds.), Multidimensional scalıng, volume I Theory New York: Seminar Press.

Carroll, J.D (1980). Models and methods for muludimensional analysis of preferential choice (or other domınance) data. In E.D. Lantermann, \& H. Feger (Eds.), Sımularıty and choice. Berlın: Hans Huber.

Carroll, J.D., DeSarbo, W S., \& De Soete, G. (1987). Stochasuc tree unfolding (STUN) models. Communication \& Cognition, 20, 63-76.

Chemukova, N.V. (1965). Algonthm for finding a general formula for the non-negauve solutions of a system of linear inequaliues. US S R Computational Mathematics and Mathematical Physics, 5, 228-233.

Chipman, J.S. (1960). Stochastac choice and subjectuve probability. In D. Willner (Ed.), Decisions, Values and Groups, volume I New York: Pergamon Press.

Coombs, Cl.H. (1950). Psychological scaling without a unit of measurement. Psychological Review, 57, 145-158.

Coombs, Cl.H. (1958). On the use of inconsistency of preferences in psychological measurement. Journal of Experımental Psychology. 55, 1-7. 
Coombs, $\mathrm{Cl} \mathrm{H}$. (1959). Inconsistency of preferences as a measure of psychological distance. In C.W. Churchman, \& P. Ratoosh (Eds.), Measurement definition and theories New York: Wiley.

Coombs, Cl.H. (1964). A Theory of Data New York: Wiley.

Coombs, Cl.H , \& Avrunin, G.S. (1977). Single-peaked preference functuons and the theory of preference. Psychological Review, 84, 216-330.

Coombs, Cl.H., Donnell, H.L., \& Kirk, D B. (1978). An expenmental study of risk preference in lottenes. Journal of Experimental Psychology Human Perception and Performance, 4, 497-512.

Coombs, Cl H., Greenberg, M.G., \& Zinnes, J.L. (1961). A double law of comparative judgment for the analysis of preferenual choice and similanty data. Psychometrika, $26,165-171$.

Cooper, L.G., \& Nakanish, M. (1983). Two logit models for external analysis of preferences. Psychometrika, 48, 607-620

Croon, M. (1983). A companson of statisucal unfolding models. Unpublished unternal report, Faculty of Social Sciences, K U Brabant.

Davd, H.A. (1988). The method of paired comparisons. New York' Hafner.

Davidson, D., \& Marschak, J (1959) Expenmental test of a stochastic decision theory In C W Churchman, \& P. Ratoosh (Eds), Measurement definttion and theortes New York: Wiley.

Davidson, R.R., \& Farquhar, P.H. (1976). A bibliography on the method of parred compansons. Bıometrics, 32, 241-252.

Davison, M. (1977). On a metric, unidimensional unfoldıng model for atutudinal and developmental data. Psychometrika, 42, 523-548.

De Soete, G., Carroll, J.D , \& DeSarbo, W S (1986). The wandenng ideal point model: A probabilisuc muludimensional unfolding model for paired compansons data. Journal of Mathematical Psychology, 30, 28-41.

De Soete, G., DeSarbo, W S., Furnas, G W., \& Carroll, J D. (1984a). Tree representations of rectangular proximity matnces. In E. Degreef \& J. Van Buggenhout (Eds.), Trends in mathematical psychology Amsterdam: North-Holland

De Soete, G., DeSarbo, W S., Fumas, G W , \& Carroll, J D. (1984b). The esumation of ultrametric and path length trees from rectangular proximity data. Psychometrika. 49, 289-310.

DeCanu, J.S. (1969). Maxımum likelihood paured comparison rankıng by linear programming. Biometrika, 56, 537-545.

DeCan, J.S. (1972). A branch and bound algonthm for maximum likelihood paired companson ranking. Biometrika, 59, 131-135.

DeSarbo, W.S., De Soete, G., \& Elıashberg, J. (1987). A new stochasuc muludımensional unfolding model for the invesugaton of of parred compansons in consumer preference/choice data. Journal of Economic Psychology, 8, 357-384.

DeSarbo, W S., De Soete, G ,\& Jedıdı, K. (1987). Probabilıstıc multudımensional scalıng models for analyzing consumer chorce behavior. Communication \& Cognition, 20, 93-116.

DeSarbo, W S., \& Hoffman, D L. (1986). Simple and weighted unfolding threshold models for the spatial representation of binary choice data. Applied Psychological Measurement, 10, 247-264. 
Dijkstra, L., Van der Eıjk, C., Molenaar, I.W., Van Schuur, W.H., Stokman, F N. \& Verhelsh, N. (1980). A discussion on stochastic unfolding. Methoden \& Data Nieuwsbrief, S, 158-175.

Dykstra, R.L. (1985). An iteratuve procedure for obtaining I-projections onto the intersecuon of convex sets. Ann. Prob , 13, 975-984.

Edgell, S.E., \& Geisler, W.S. (1980). A set-theoret1c random utility model of choice behavior. Journal of Mathematical Psychology, 21, 265-278.

Edgell, S.E., Geisler, W.S., \& Zinnes, J.L. (1973). A note on a paper by Rumelhart and Greeno. Journal of Mathemanical Psychology, 10, 86-90.

Eisler, H., Holm, S., \& Montgomery, H. (1979). A general psychophysical differential equation: a companson of three specifications. Journal of Mathematical Psychology, $20,16-34$.

Falmagne, J.C. (1971). The generalized Fechner problem and discrimination. Journal of Mathematical Psychology, 8, 22-43.

Fishburn, P.C. (1964). Decision and value theory New York: Wiley.

Fishburn, P.C. (1973). Binary choice probabilities: on the varieties of stochastic transiuvity. Journal of Mathematical Psychology, 10, 327-352.

Fishburn, P.C. (1977). Models of individual preference and choice. Synthese, 36, 287-314.

Flueck, J.A., \& Korsh, J.F. (1974). A branch search algonthm for maxımum likelıhood paired companson ranking. Biometrika, 61, 621-626.

Flueck, J.A., \& Korsh, J.F. (1975). A generalızed approach to maxımum likelıhood paired companson rankang. Annals of Statistics, 3, 846-861.

Gaul, W. (1987). Probabilisuc choice behavior models and their combination with additional tools needed for applications to marketung. Communication \& Cognition, $20,77-92$.

Gebhardt, F. (1970). An algonthm for monotone regression with one or more dependent vanables. Biometrika, 57, 263-271.

Georgescu-Roegen, N. (1958). Threshold in choice and the theory of demand. Econometrika, 26, 157-168.

Greenberg, M.G. (1965). A method of successive cumulations for the scaling of paircomparison preference judgments. Psychometrika, 30, 441-448.

Gumbel, EJ. (1961). Sommes et différences de valeurs extrêmes indépendentes. Comptes Rendus de l' Académie des Sciences, Parts, 253, 2838-2839.

Halff, H.M. (1976). Choice theories for differentially comparable alternatives. Journal of Mathemancal Psychology, 14, 244-246.

Hays, W.L., \& Benneth, J.F. (1961). Multudimensional unfolding: Determining configuration from complete rank order preference data. Psychometrika, 26, 221-238.

Hefner, R.A. (1958). Extensions of the law of comparative judgment to discriminable and multidimensional stımuli. Doctoral Dissertation, University of Michigan.

Heıser, W J., \& De Leeuw, J. (1981). Muladimensional mapping of preference data. Mathémattques et Sciences Humaines, 73, 39-96.

Heıser, WJ. (1981). Unfolding analysis of proximity data. Doctoral Dissertation, R.U. Leiden.

Hubert, L. (1976a). Seriation using asymmetnc proximity measures. Britush Journal of Mathematical and Statistical Psychology, 29, 32-52. 
Hubert, L. (1976b). Quadratic assignment as a general data analysis strategy. British Journal of Mathematical and Statistical Psychology, 29, 190-241.

Hubert, L., \& Schultz, J. (1976). Maximum likelihood paired-comparison ranking and quadratic assignment. Biometrika, 62, 655-659.

Indow, T. (1975). On choice probability. Behaviormetrika, 2, 13-31.

Jansen, P.G.W. (1981). Spezifisch objektive Messung im Falle nicht-monotoner Einstellungsitems. Zeitschrif für Sozialpsychologie, 12, 169-185.

Jansen, P.G.W. (1983). Rasch analysis of attitudinal data. Doctoral Dissertation. K.U. Nijmegen.

Kendall, M.G. (1955). Further contributions to the theory of paired comparisons. Biometrika, 11, 43-62.

Kendall, M.G., \& Smith, B.B. (1939). On the method of paired comparisons. Biometrika, 31, 324-345.

Krantz, D.M. (1964). The scaling of small and large color differences. Ph.D. Thesis, University of Pennsylvania. Ann Arbor: University Microfilms.

Krantz, D.M. (1967). Rational distance functions for multidimensional scaling. Journal of Mathematical Psychology, 4, 226-245.

Krantz, D.H., Luce, R.D., Suppes, P., \& Tversky, A. (1971). Foundations of measurement theory. Vol 1 . New York: Academic press.

Kruskal, J.B. (1964). Multidimensional scaling by optimizing goodness of fit to a nonmetric hypothesis. Psychometrika, 29, 1-27.

Luce, R.D. (1959). Individual choice behavior. New York: Wiley.

Luce, R.D. (1961). A choice theory analysis of similarity judgments. Psychometrika, 26, 151-163.

Luce, R.D., \& Raiffa, H. (1957). Games and Decisions. New York: Wiley.

Luce, R.D., \& Suppes, P. (1965). Preference, utility and subjective probability. In R.D. Luce, R.R. Bush, \& E. Galanter (Eds.), Handbook of mathematical psychology, vol. III. New York: Wiley.

Marschak, J. (1960). Binary choice constraints and random utility indicators. In K.J. Arow, S. Karlin, \& P. Suppes. (Eds.), Mathematical models in the social sciences. Stanford: Stanford University Press.

May, K.O. (1954). Intransitivity, utility, and the aggregation of preference patterns. Econometrika, 22, 1-13.

McClelland, G.H., \& Coombs, Cl.H. (1975). ORDMET: A general algorithm for constructing all numerical solutions to ordered metric structures. Psychometrika, 40, 269-290.

Mood, A.M., Graybill F.A., \& Boes D.C. (1974). Introduction to the theory of statistics. Kogakusha: Mc Graw-Hill.

Morrison, H.W. (1963). Testable conditions for triads of paired comparisons choices. Psychometrika, 28, 369-390.

Nagels, L.P.G., \& Elzinga, C.H. (1984). Beschrijving van diverse algoritmen vereist voor het vinden van de oplossingen van een stelsel lineaire ongelijkheden en voor het kiezen van een optimale representant uit die oplosssingen. Internal publication, Mathematical Psychology Group, K.U. Nijmegen.

Patnaik, P.B. (1949). The non-central $\mathrm{X}^{2}$ and $F$ distributions and their applications. Biometrika, 36, 202-232. 
Pendergrass, R.N., \& Bradley, R.A. (1960). Rankıng in tnple compansons. In I Olkın, S Ghurye, W. Hoffding, W. Madow, \& H Mann (Eds ), Contributions to probability and statistics Stanford. Stanford Unversity Press.

Phillips, J.P.N. (1967). A procedure for determuning Slater's $ı$ and all nearest adjoining orders. British Journal of Mathematical and Statistical Psychology, 20, 217-225.

Phillips, J.P.N. (1969). A further procedure for determining Slater's $\imath$ and all nearest adjoining orders. British Journal of Mathematical and Statistical Psychology, 22, 97-101.

Phillips, J P N. (1971). A note on representation of ordered metnc scalıng. Brittsh Journal of Mathematical and Statistical Psychology, 24, 239-250

Ramsay, J.O. (1969). Some statstical consıderations in multıdımensional scalıng Psychometrika, 34, 167-182

Ramsay, J.O. (1977). Maxımum likelihood estımation in muludımensional scalıng. Psychometrika, 42, 241-266.

Ramsay, J.O. (1978a). Confidence regions for multudimensional scaling analysıs. Psychometrika, 43, 145-160.

Ramsay, J O (1978b). MULTISCALE Four programs of multidimensional scaling by the method of maximum likelihood Chicago International Educational Services

Ramsay, J.O (1980a). Some small sample results for maxımum likelihood multudımensional scaling. Psychometrika, 45, 141-146.

Ramsay, J O. (1980b). The joint analysis of direct ratings, pairwise preferences and dissimilanties. Psychometrika, 45, 149-166.

Ramsay, J O. (1982). Some statısucal dpproaches to muludimensional scalıng data. Journal of the Royal Statistical Society, A, 149, 285-312.

Ranyard, R H. (1976). An algonthm for maxımum lıkelıhood rankıng and Slater's $ı$ from paured comparisons. Britush Journal of Mathematical and Statistical Psychology, 29. 242-248.

Remage, R., \& Thompson, W.A (1966). Maximum likelihood paired companson rankings. Btometrika, 53, 143-149.

Restle, F. (1961). Psychology of judgment and choice New York: Wiley

Ruce, W.R (1988). A new probability model for determining exact $P$-values for $2 \times 2$ contungency tables when companing binomial proportions. Biometrics, 44, 1-22.

Roberts, F.S. (1971). Homogeneous families of semiorders and the theory of probabilisuc consistency. Journal of Mathematical Psychology, 8, 248-263.

Robertson, T., Wnght, F.T., \& Dykstra, R L. (1988). Order restricted stanstical inference. New York: John Wiley \& Sons.

Roskam, E.E.Ch.I. (1968). Metric analysis of ordinal data in psychology Voorschoten VAM.

Roskam, E.E.Ch.I. (1987). ORDMETJ. An improved algorithm to find the maximin solution to a system of linear inequalities Internal report 87 MA 06, Mathematical Psychology Group, K.U. Nıjmegen.

Ross, R.T. (1934). Optumum orders for the presentation of pars in the method of paired compansons. Journal of Educational Psychology, 25, 375-382.

Rumelharr, D L., \& Greeno, J.G. (1971). Similanty between stimuli: an expenmental test of the Luce \& Restle choice axioms. Journal of Mathemantical Psychology, 8, 370-381. 
Savage, LJ. (1951). The theory of statistical decision.Journal of the American Statistical Association, 46, 55-57.

Schönemann, P.H. (1970). On metric multidimensional unfolding. Psychometrika, 35, 349-366.

Schönemann, P.H., \& Wang, W.M. (1972). An individual differences model for the multidimensional analysis of preference data. Psychometrika, 37, 275-309.

Scoth D. (1964). Measurement models and linear inequalities. Journal of Mathematical Psychology, 1, 233-247.

Scoth D., \& Suppes, P. (1958). Foundational aspects of theories of measurement. Journal of Symbolic Logic, 23, 113-128.

Shepard, R.N. (1957). Stimulus and response generalization: a stochastic model relating generalization to distance in psychological space. Psychometrika, 22, 325-345.

Shepard, R.N. (1958). Stimulus and response generalization: deduction of the generalization gradient from a trace model. Psychological Review, 65, 242-256.

Shepard, R.N. (1962). The analysis of proximities: MDS with an unknown distance function. Psychometrika, 27, 125-140; 219-245.

Sherif, M., \& Sherif, C.W. (1967). The own categories procedure in attinde research. In M. Fishbein (Ed.), Readings in attitude theory and measurement. New York: John Wiley \& Sons.

Sherif, M., \& Sherif, C.W. (1969). Social Psychology. New York: Harper \& Row, Tokyo: Weatherhill.

Singh, J. (1973). Paired comparison rankings by linear programming. Communications in Statistics, 1, 351-364.

Singh, J., \& Thompson, W.A. (1968). A treatment of ties in paired comparisons. Annals of Mathematical Statistics, 39, 2002-2105.

Sixtl, F. (1973). Probabilistic unfolding. Psychometrika, 38, 235-248.

Slater, P. (1961). Inconsistencies in a schedule of paired comparisons. Biometrika, 48, 303-312.

Smith, A.F.M., \& Payne, C.D. (1974). An algorithm for deteremining Slater's $i$ and all nearest adjoining orders. British Journal of Mathematical and Statistical Psychology, $27,49-52$.

Srinivasan, V., \& Shocker, A.D. (1973). Linear programming techniques for multidimimensional analysis of preferences. Psychometrika, 1973, 38, 337-369.

Suppes, P. (1962). Models of data. In E. Nagel, P. Suppes, \& A. Tarski (Eds.), Logic, methodology and philosophy of science. Proceedings of the 1960 International Congress. Stanford, California: Stanford University Press.

Suppes, P., \& Zinnes, J.L. (1963). Basic measurement theory. In R.D. Luce, R.R. Bush, \& E. Galanter (Eds.), Handbook of mathematical psychology, vol.I. New York: Wiley.

Takane, Y., \& Carroll, J.D. (1981). Nonmetric maximum likelihood multidimensional scaling for directional rankings of stimuli. Psychometrika, 46, 389-405.

Takane, Y., Bozdogan, H., \& Shibayama, T. (1987). Ideal point discriminant analysis. Psychometrika, 52, 371-392.

Takane, Y.(1981). Multidimensional successive scaling: a maximum likelihood method. Psychometrika, 46, 9-28.

Takane, Y. (1987). Analysis of covariance structures and probabilistic binary choice data. Communication \& Cognition, 20, 45-62. 
Thompson, W.A., \& Remage, R (1964). Rankıngs from paired compansons. Annals of Mathematical Statistics, 35, 739-747.

Thurstone, L.L. (1927). A law of comparatuve judgment. Psychological Review, 34, 237-286.

Thurstone, L.L. (1959). The measurement of values Chicago: University of Chicago Press.

Torgerson, W.S. (1958). Theory and methods of scaling New York: Wiley.

Tucker, L.R. (1960). Intra-individual and inter-individual muludimensionality. In H. Gulluksen, \& S. Messick (Eds.), Psychological Scalıng Theory and applicallons New York: Wiley

Tversky, A. (1969) Intransituvity of preferences. Psychological Review, 76, 31-48.

Tversky, A. (1972a). Choice by elımination Jounal of Mathematical Psychology, 9, 341-367.

Tversky, A. (1972b). Elımination by aspects a theory of choice. Psychologıcal Review, 79, 31-48.

Tversky, A., \& Russo, J.E. (1969). Substututability and simılanty in binary choices Journal of Mathematical Psychology, 6, 1-12.

Tversky, A., \& Sattath, S (1979). Preference trees. Psychological Review, 86, 542-573.

Van Eeden, C. (1956). Maximum likelıhood estımation of ordered probabilities. Proc

Kon Ned Akad Wet (a), 59, Indag Math, 18, 444-455.

Van Eeden, C. (1957a) Maxımum likelıhood esumates of partually or completely ordered parameters I Proc Kon Ned Akad Wet (a), 60, Indag Math, 19, 128-136

Van Eeden, C. (1957b). Maxımum likelihood esumates of partially or completely ordered parameters II. Proc Kon Ned Akad Wet (a), 60, Indag Math, 19, 201-211.

Van Schuur, W H. (1984). Structure in political beliefs A new model for stochastic unfolding with application to European party activists Doctoral dissertation, R U Groningen.

Wang, W.M., Schonemann, P.H., \& Rusk, J G. (1975). A conjugate gradient algonthm for the muladimensional analysis of preference data. Multivariate Behavioral Research, 10, 45-99.

Zınnes, J.L., \& Gnggs, R.A (1974). Probabılıstıc multıdımensıonal unfoldıng analysis. Psychometrika, 39, 327-350.

Zınnes, J.L., \& MacKay, D B. (1983). Probabilıstıc multıdımensıonal scalıng: complete and incomplete data. Psychometrika, 48, 27-48. 


\section{Appendix A PSTRIX}

This appendix contains a selection of FORTRAN routines from the PSTRIX package. PSTRIX was written as an implementation of the evaluation strategy described in Chapter 4. The complete package is available from the author.

\footnotetext{
[PSTRIX.DOC]

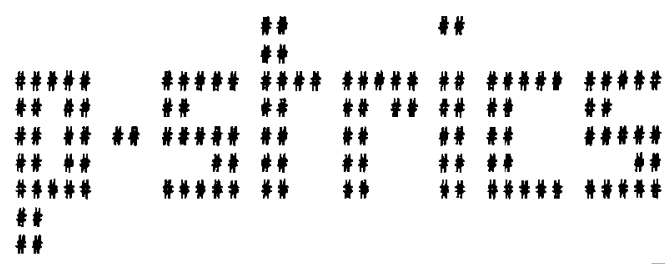

Solves seriation problems with ordinal restrictions on binomial prooabilities

(C) PMM Bossuyt

Center for Clinical Decision Analysis

Version $\checkmark 3.57$

Erasmus University

January 7, 1990

PO BOX 1738

3000 DR Rotterdam

The Netherlands

Summary

Conditions supported

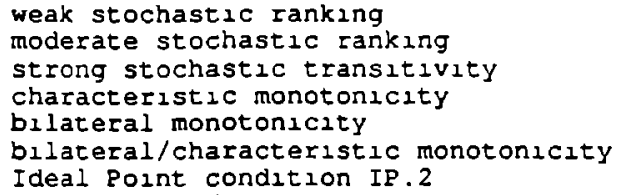


Program flow

The main program is called PSTRIX. Apart from calls to entries in UTILS, this program first calls GTDATA. This routine opens, reads and checks the infile specified on the command line, and opens the data and output files if necessary. PSTRIX then calls INIT. This routine formulates the restrictions, in terms of the indices of the ranking, and generates initial rankings for the isotonic regression algorithm, also in terms of the indices of the ranking. GTDATA reads the input frequencies, calculates the unconditional estimates and does some housework. PSTRIX then passes control to a specific subroutine, conditional on the condition studied (CHWST, CHMST, ....).

The majority of these subroutines are highly similar. They initialize the solution storage, and the branching subroutine (BRANCH), look for an initial solution and then wander through the ranking tree, calculating the lower bound of the objective function for the corresponding subset of rankings. This is done by passing the vector that translates the ranising indices to the original indices (1.e. the indices of the data $f i l e$ ). The routines calls CHSOL to check, store and write the solutions.

POITIng PSTRIX

Porting the program to other systems should not prove to be too difficult. The program does not use strict FORTRAN 77 syntax, but makes only moderate use of extensions, mostly in the declarations (e.g REAL*8, INTEGER*2) All device-speciflc routines are grouped under UTILS.FOR, except for some code in GTPARS.FOR to parse the command ine. In the code included in this package, there is no mixing of languages, and ANSI escape sequences are used to control screen colors and cursor positioning. These routines can be replaced by others (MASM, C, ...) for improved efficiency and speed.

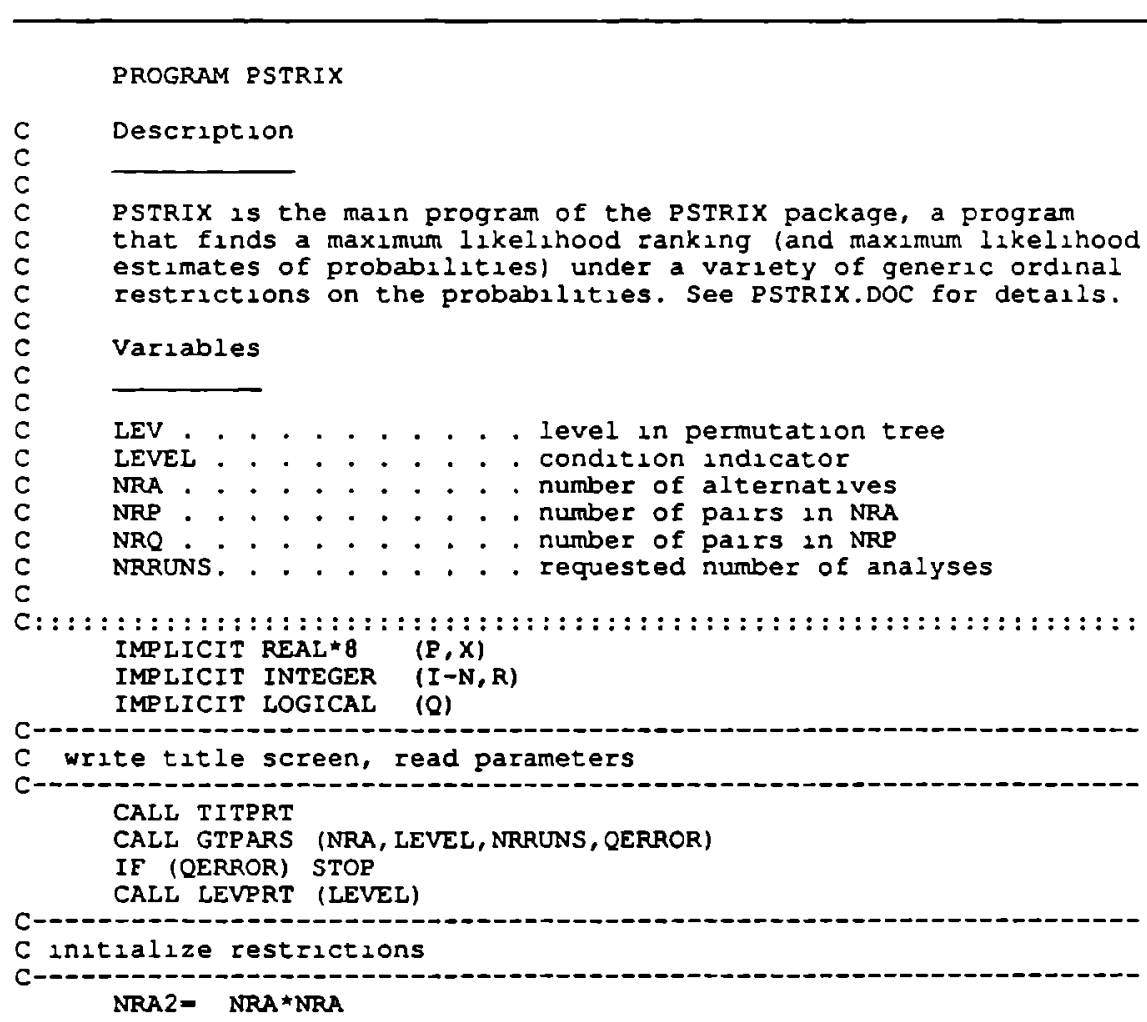




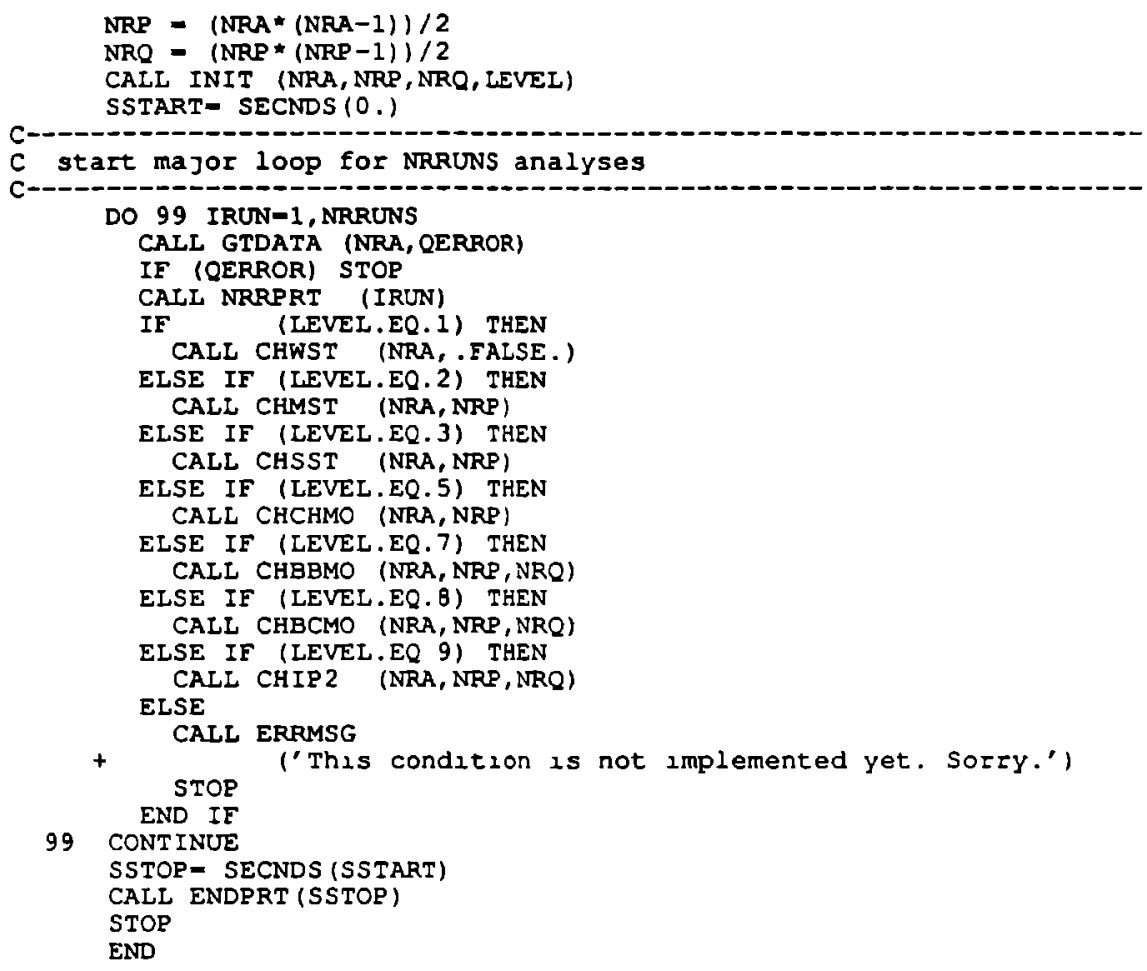

BLOCK DATA PBLOCK

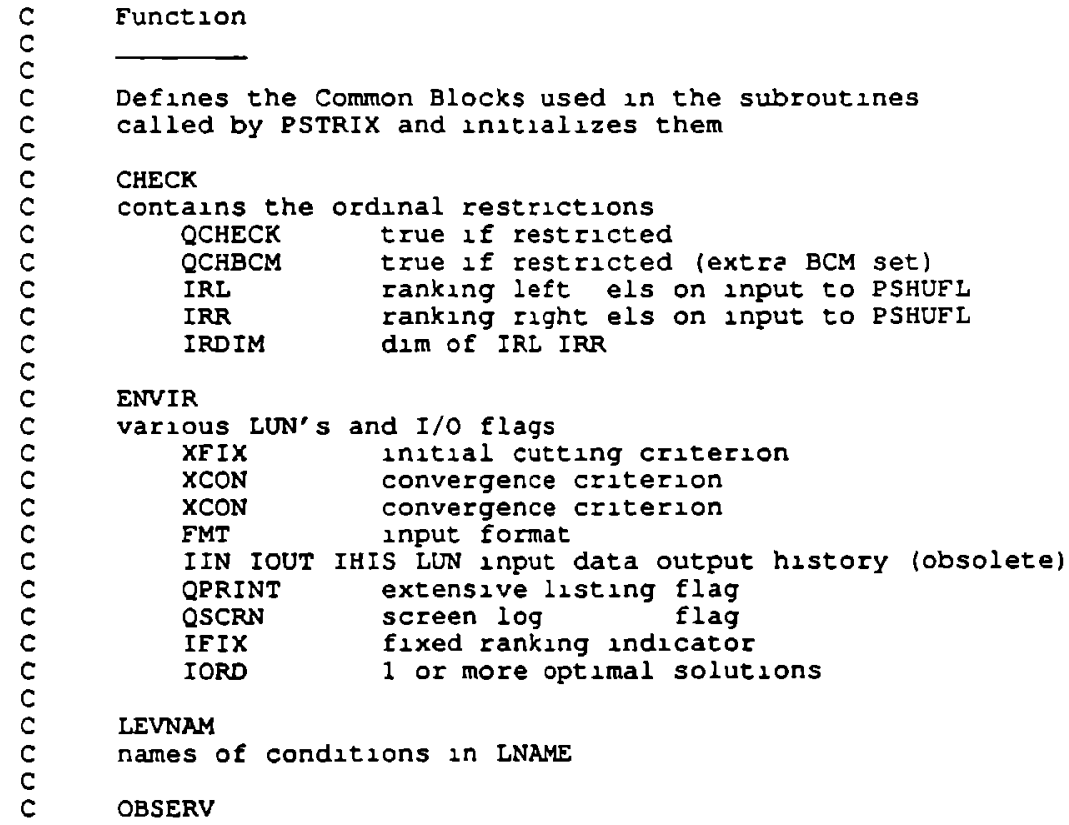




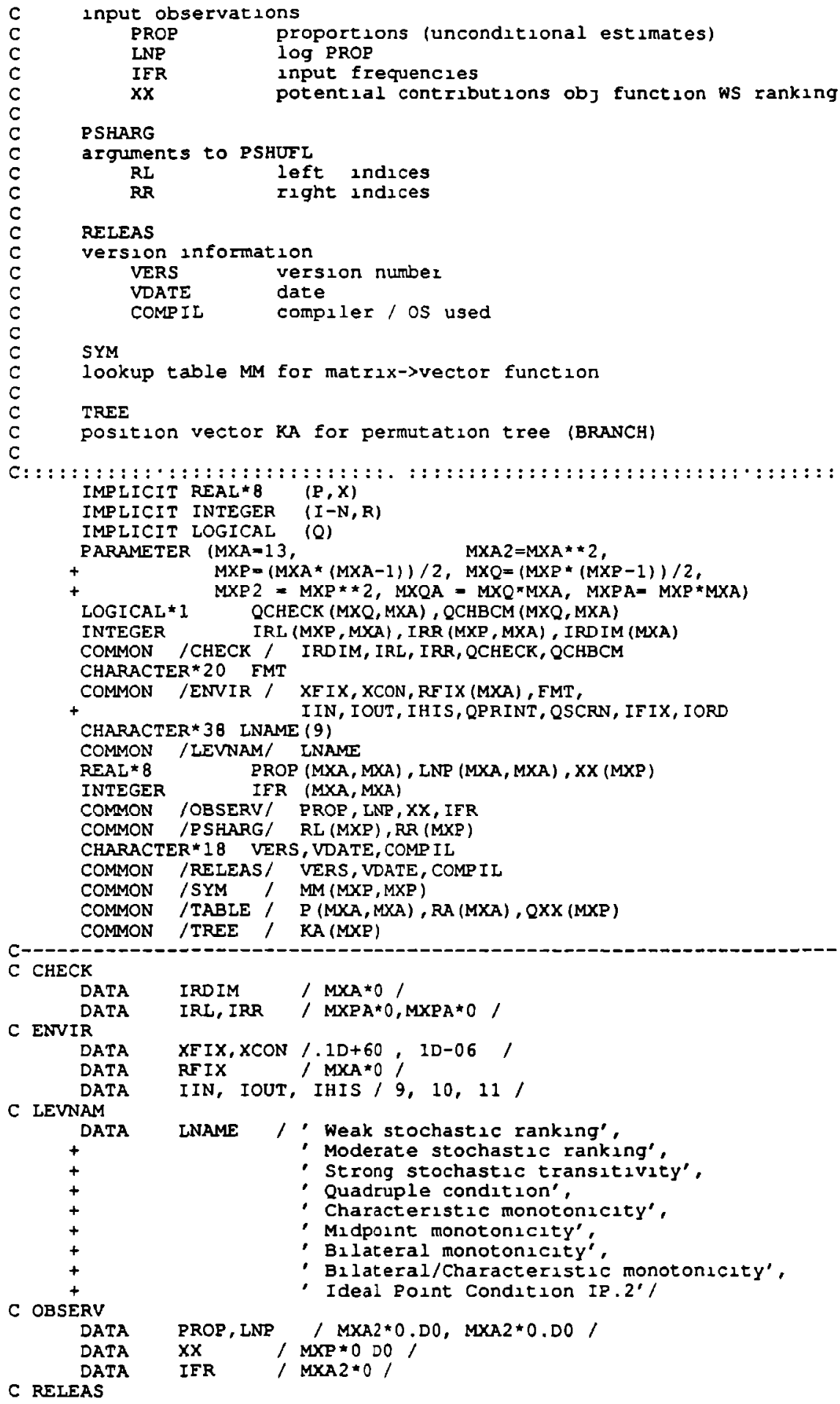




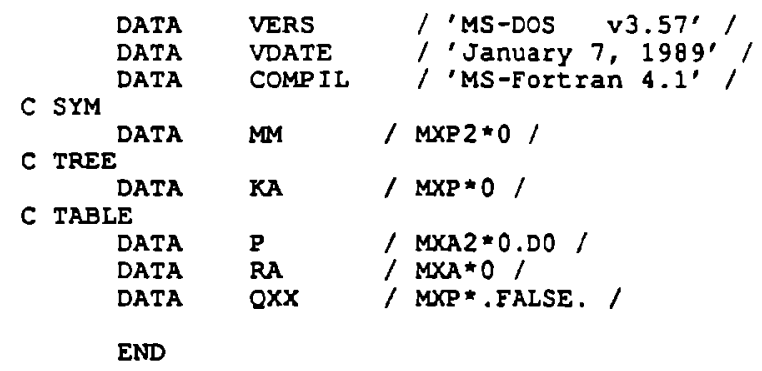

SUBROUTINE INIT (NRA, NRP, NRQ, LEVEL)

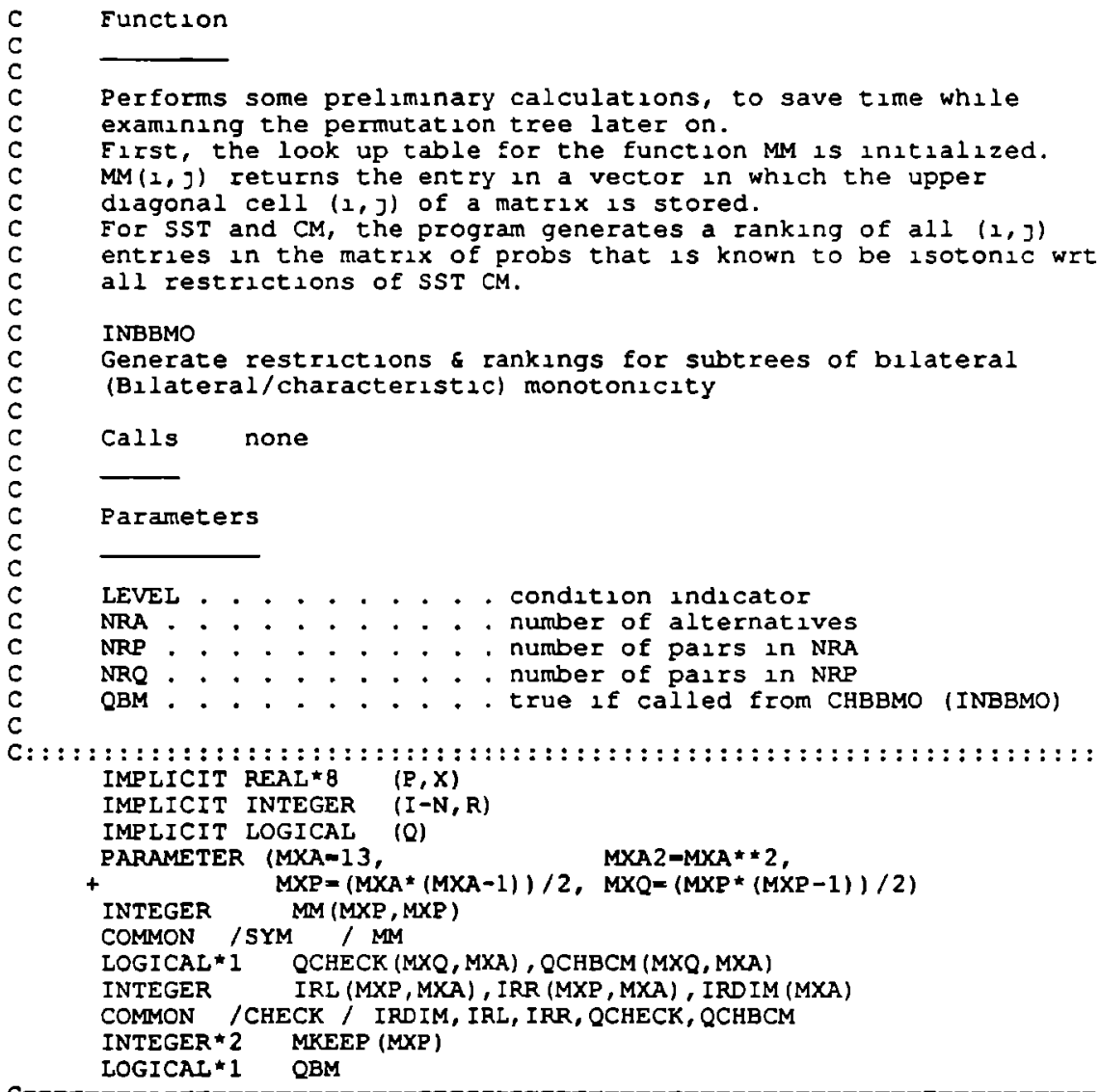

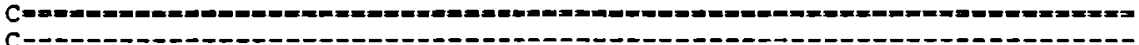

C 0. Initialize look-up table (all routines)

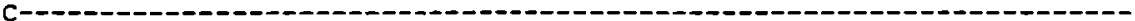

$K=0$

DO $02 I=2$, NRP

$I I=I-1$

$K=K+I I-1$

DO $02 \mathrm{~J}=1, \mathrm{II}$

$M M(I, J)=K+J$

$M M(J, I)=K+J$ 
02 CONTINUE

IF (LEVEL.GT.6) RETURN

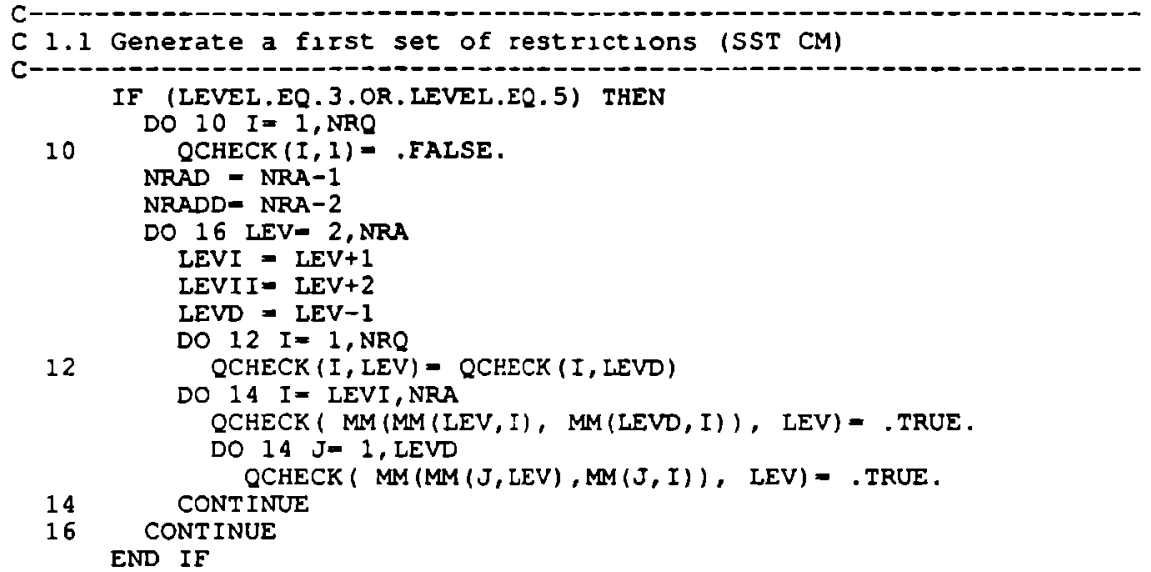

C 1.2 .1 Find the initial ranking (MST (2nd procedure)

IF (LEVEL.EQ . 2.OR. LEVEL.EQ.3) THEN

DO 24 LEV $=1$, NRA

$I 3=0$

DO 22 II I LEV , 1, - 1

$\mathrm{J} 2=I 1+1$

DO 22 I $2=J 2$, NRA

$\mathrm{IJ}=\mathrm{I} 3+1$

$\operatorname{IRL}(\mathrm{I3}, \mathrm{LEV})=$ I1

$22 \operatorname{IRR}($ I 3, LEV $)=$ I 2

CONTINUE

$\operatorname{IRDIM}($ LEV) $=I 3$

24 CONTINUE

IF (LEVEL.EQ.2) RETURN

ELSE IF (LEVEL.EQ.5) THEN

C 1.2 .2 Find the initial ranking (CM)

DO $34 L E V=1, N R A$

I $3=0$

DO $32 I 1=1, L E V$

$\mathrm{J} 2=I 1+1$

DO 32 I $2=\mathrm{J} 2, \mathrm{NRA}$

I $3=13+1$

IRL ( I 3, LEV) = II

32 CONTINUE

IRR ( I 3, LEV) = I2

$\operatorname{IRDIM}(\mathrm{LEV})=$ I3

34 CONTINUE

END IE

GOTO 70

C 2. ENTRY FOR BILATERAL \& CHARACTERISTIC MONOTONY

ENTRY INBBMO (INDEX, NRA, NRQ, QBM)

NRAD $=$ NRA-I

NRADD $=$ NRA -2

INDEXI $=$ INDEX $+I$

INDEXD - INDEX-1

C 2.1 Generate a first set of restrictions (BM BCM)

DO 41 I = 1, NRQ

DO 41 QCHBCM $(I, 1)=$. FALSE.

41 QCHECK $(I, 1)$-.FALSE. 


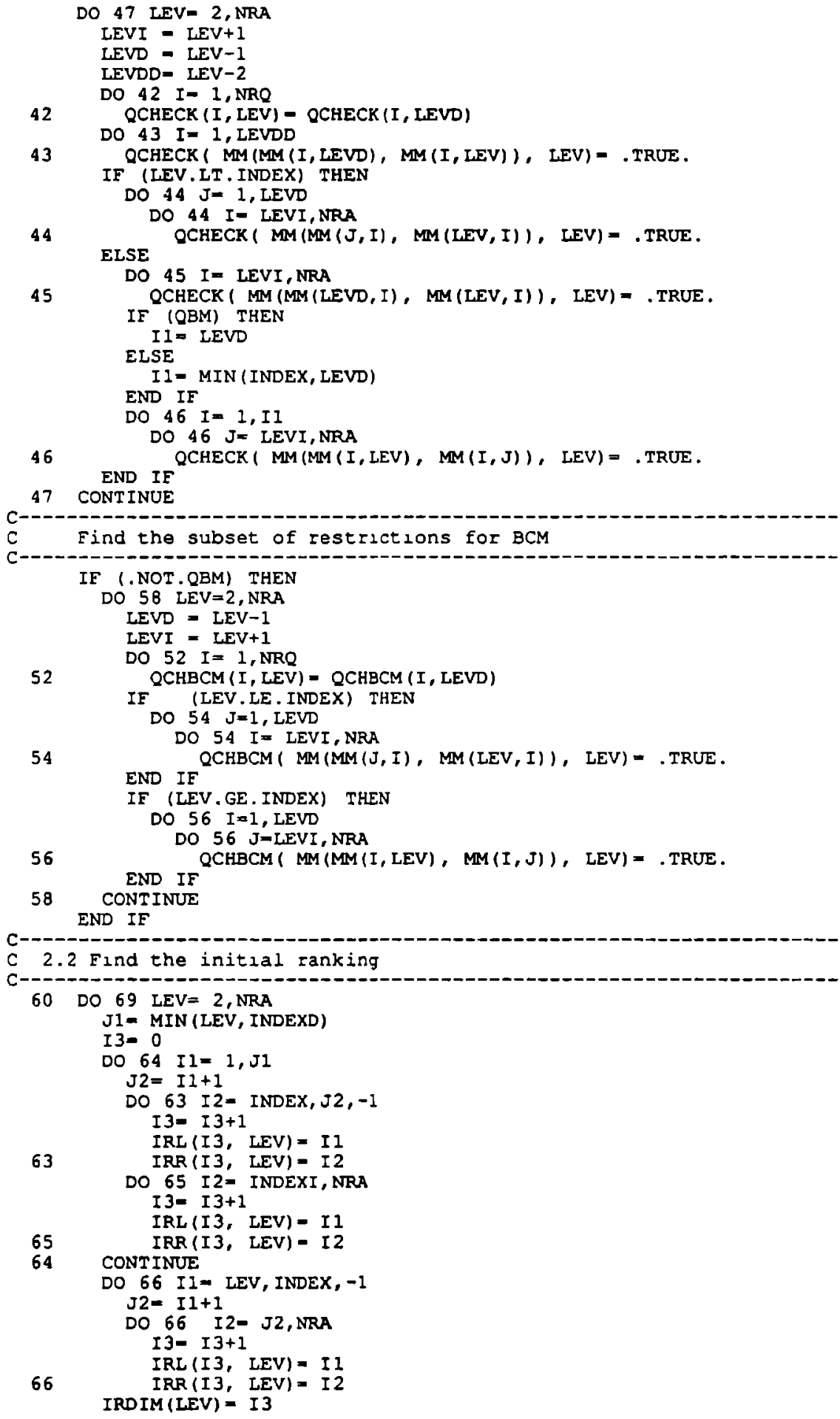




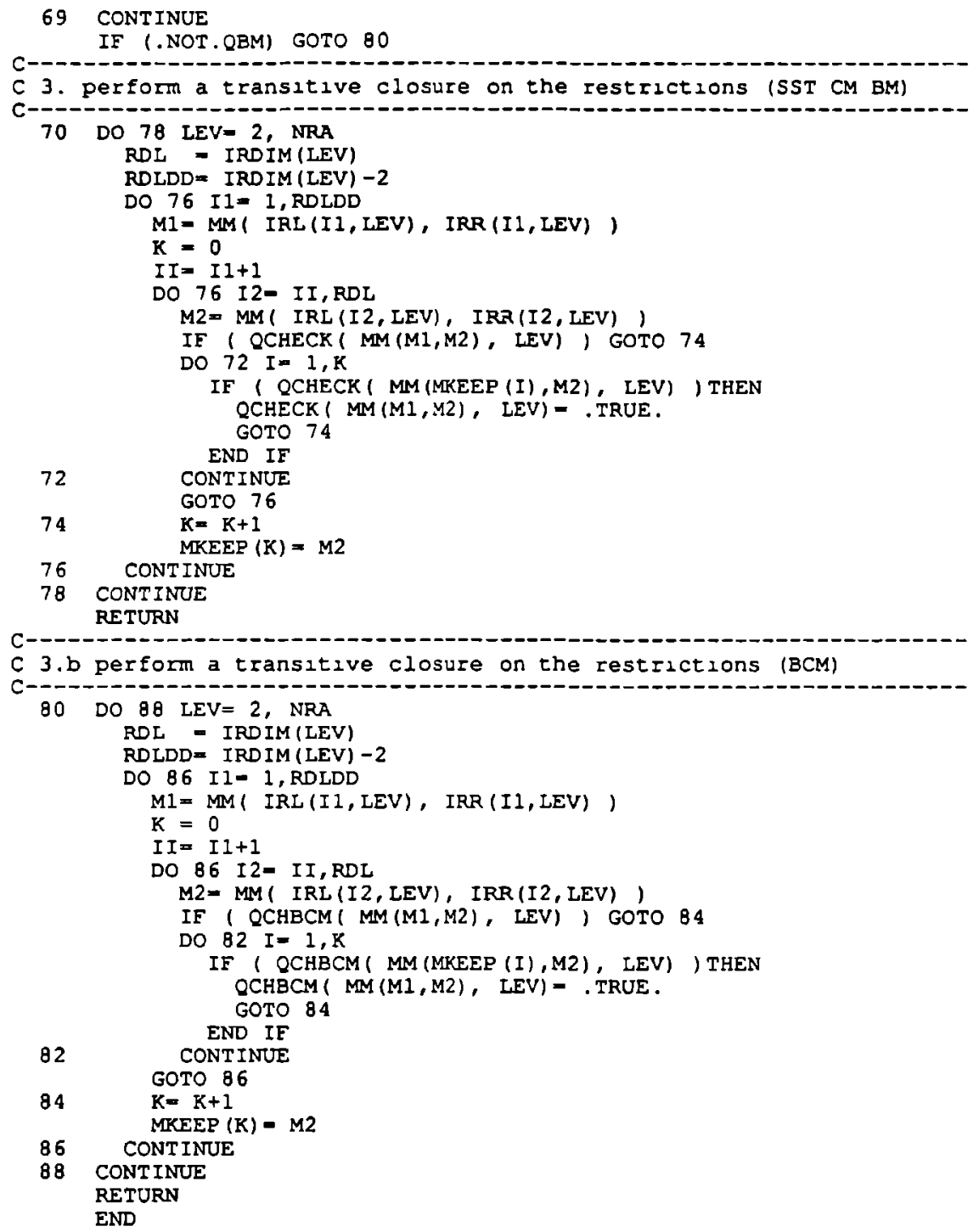


Parameters

NRA . . . . . . . . number of alternatives

NRP . . . . . . . . number of pairs in NRA

Local variables

LEV . . . . . . . . level in permutation tree

Q50 . . . . . . . . check >.5 flag (RSHUFL)

RDIM. . . . . . . . length of RL RR (passed to PSHUFL)

XPERM . . . . . . . . value of obj function

XDIFF . . . . . . . . convergence criterion

xSOL. . . . . current solution value obj function

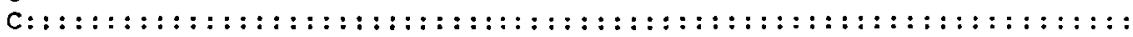

IMPLICIT REAL* $B$ (P, $X)$

IMPLICIT INTEGER (I-N,R)

IMPLICIT LOGICAL (Q)

PARAMETER (MXA=13, $\quad$ MXA2 $=M \times A * * 2$,

$+\quad M X P=(M X A *(M \times A-1)) / 2, M X Q=(M X P *(M X P-1)) / 2)$

PARAMETER (MXTR= $(M \times A *(M X A-1) *(M X A-2)) / 6, M X I T E R=500)$

CHARACTER 20 FMT

COMMON /ENVIR / XEIX, XCON, RFIX (MXA), FMT,

$+$

I IN, IOUT, IHIS, QPRINT, QSCRN, IF IX, IORD

REAL * $8 \quad$ PROP (MXA, MXA), LNP (MXA, MXA), XX (MXP)

INTEGER IFR (MXA, MXA)

COMMON /OBSERV/ PROP, LNP, XX, IFR

COMMON /SYM / MM (MXP, MXP)

LOGICAL*1 QCHECK (MXQ, MXA), OCHBCM (MXQ, MXA)

INTEGER IRL (MXP, MXA), IRR (MXP, MXA), IRD IM (MXA)

COMMON /CHECK / IRDIM, IRL, IRR, QCHECK, QCHBCM

COMMON /TABLE / P (MXA, MXA), RA (MXA), QXX (MXE)

COMMON /PSHARG/ RL (MXP), RR (MXP)

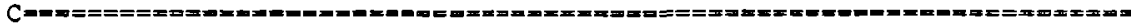

C () Prepare

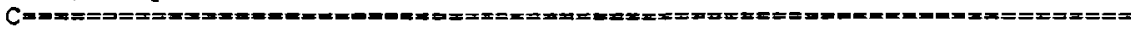

NRAD- NRA-1

Q50 - . TRUE.

IF (QSCRN) CALL CLRPRT

C---

C determine the initial permutation; find the ML dominance ranking

C if no initial permutation is specified

IF (IFIX.EQ.0) THEN

CALL CHWST (NRA, . TRUE.)

CALL SOLFIL (NRA)

ELSE

DO $10 I=1$, NRA

$10 \quad R A(I)=\operatorname{RFIX}(I)$

END IF

C-c-

Cinitialize the branching subroutine, initialize solution storage,

CALL INIBRA (NRA)

CALL INISOL (NRA)

XSOL- XFIX

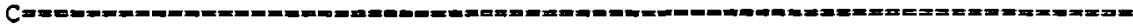

C () Start search through permutation tree (loop between 30 and 40 )

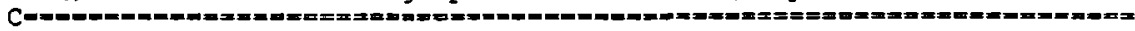

IF (XFIX.LT.1.D+30) THEN

$L E V=2$

ELSE

LEV = NRAD

END IF

C----

C Initialize estimates, pool indicator, increment level if necessary

30 IF (LEV.EQ.1) LEV LEV+1 
IE (QSCRN) CALL RAPRT (RA, LEV)

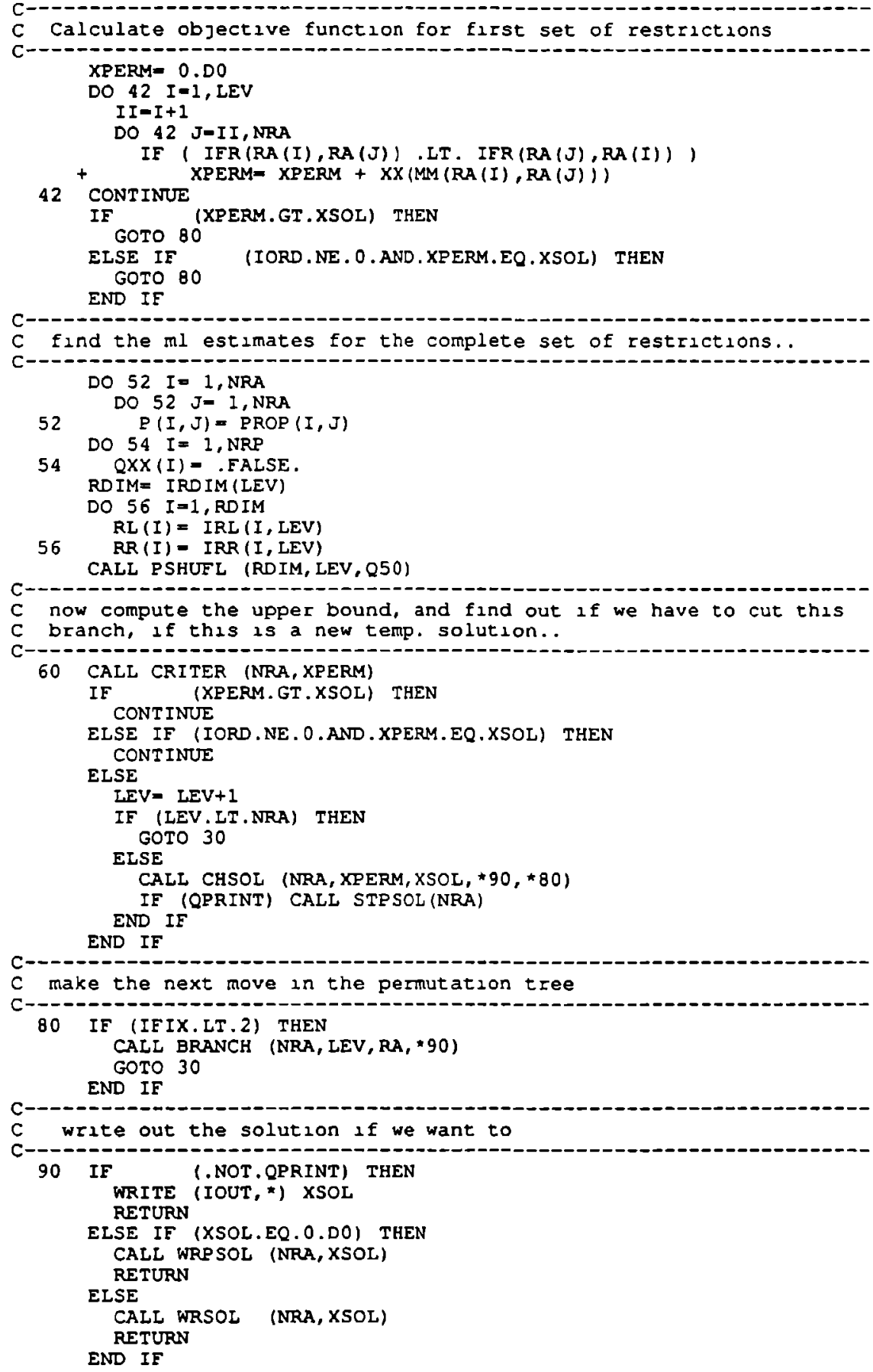


SUBROUTINE PSHUFL (RDIM, LEV, Q50)

Function

Finds the maximum likelihood estimates of the binomial probabilities under ordinal restrictions.

These restrictions are conditional on a ranking of the alternatives. RA(1) returns the element currently at position 1 in this ranking. Whether or not an ordinal restriction exists can be determined by examining QCHECK. Entries in QCHECK contain matrix-to-vector translations $M$, based on the position indices

$\operatorname{QCHECK}[M M(M M(1, j), M M(1, k))]=. T R U E$.

means that a restriction exists between

$P[R A(1), R A(])] \leq P[R A(1), R A(k)]$

The observed frequencies are in the common block DATA: $\operatorname{IFR}(1, j)$. The unconditional estimates are also in the common block DATA: PROP $(2, j)$. Initially, all palrs $(1, j)$ are organized in a QCHECK-1sotonic ranking, indicated by the vectors RL (left) and RR (IIght).

$\operatorname{QCHECK}[\operatorname{MM}(\operatorname{MM}(R L(1), R R(1)), \operatorname{MM}(R L(k), R R(k)))]=$.TRUE.

$\Rightarrow 1<k$

The pair of vectors RL(1),RR(J) return the left, right element resp. at position at position 1 in the ranking. These are not the original indices, but positions in the ranking of the alternatives. The original lndices are returned by RL(RA(1)). The algorithm will modify the ranking, but it has to remain oCHECK-consistent.

The set $Y$ of pairs is always partitioned into $T 1$ and $T 2$. All elements 1-INP are in $T 1$, the remaining in $T 2$. Within $T 1$, the $\mathrm{ml}$ estimates, conditional on the subset of restrictions for $\mathrm{T} 1$ only, have been found; furthemore, the ranking of the couples is isotonically related to a ranking of the corresponding (conditional) estimates. Thes estimates are stored in P.

The subset T1 of all ordered couples [RL(1), RR(1)] is partitioned in a number of solution blocks. In a solution block, the estimates for all couples are equal to the weighted average of the unconditional estimates (weighted by the number of frequencies). The algorithm tries to partition the solution blocks as far as possible, without violating the ordinal restrictions.

The QPOOL flags for the solution blocks work as follows : QPOOI (1) is true if the couples at positions $1-1$ and 1 are in the same solution block.

Example: assume that $(1,2,3\}\{4,5\}\{6\}\{7,8,9,10\}\{11,12\}$ are the solution blocks

$$
\begin{aligned}
& 1-2-3 \quad 4-5 \quad 6 \quad 7-8-9-10 \quad 11-12
\end{aligned}
$$

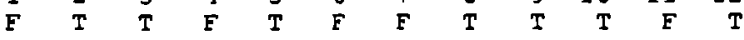

Calls

none 


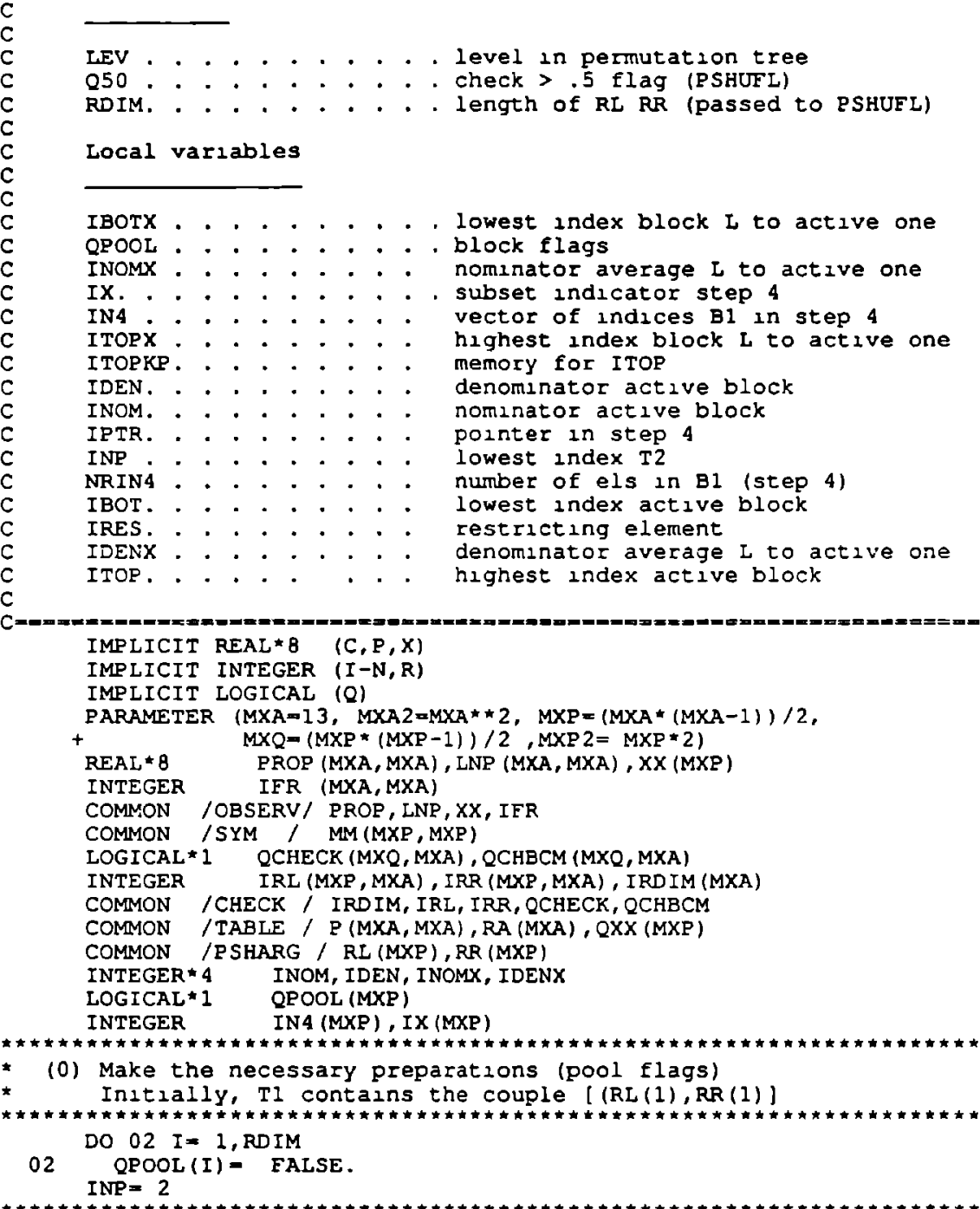

(1) Examine the restrictions between the couples in $T 1$ and the - active couple [RL (INP), RR (INP)]

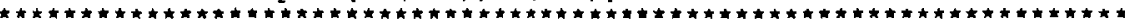
10 INPD $=$ INP -1

C Check whether there exists a restriction between [RL (INP), RR (INR)] $C$ and one the couples currently in Tl,

$C$ - if there is none, insert [RL(INP), RR(INP)] based on the value of

C Its unconditional estimate $\quad$ (12)

C - if there is one, insert [RL (INP), RR (INP)] to the Iight of the C solution block with the restricting element [RL (IRES), RR(IRES)]

C

$M I=M M(R L$ (INP), RR (INP) )

DO 11 I= INPD, $1,-1$

IF (.NOT.OCHECK( MM(MM(RL(I),RR(I)),MI), LEV) ) GOTO 11 IRES - I

IRESI $I+1$ 


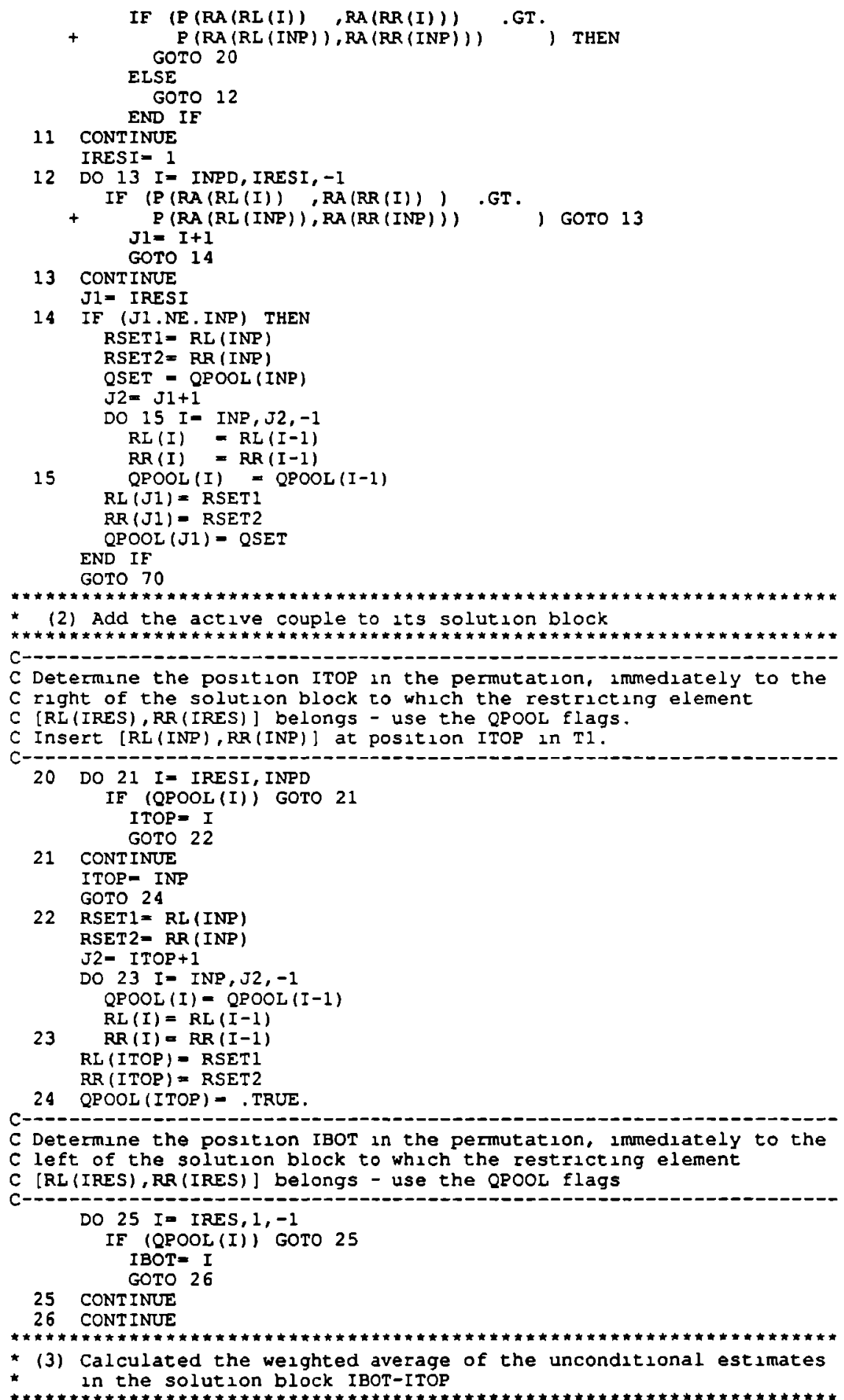




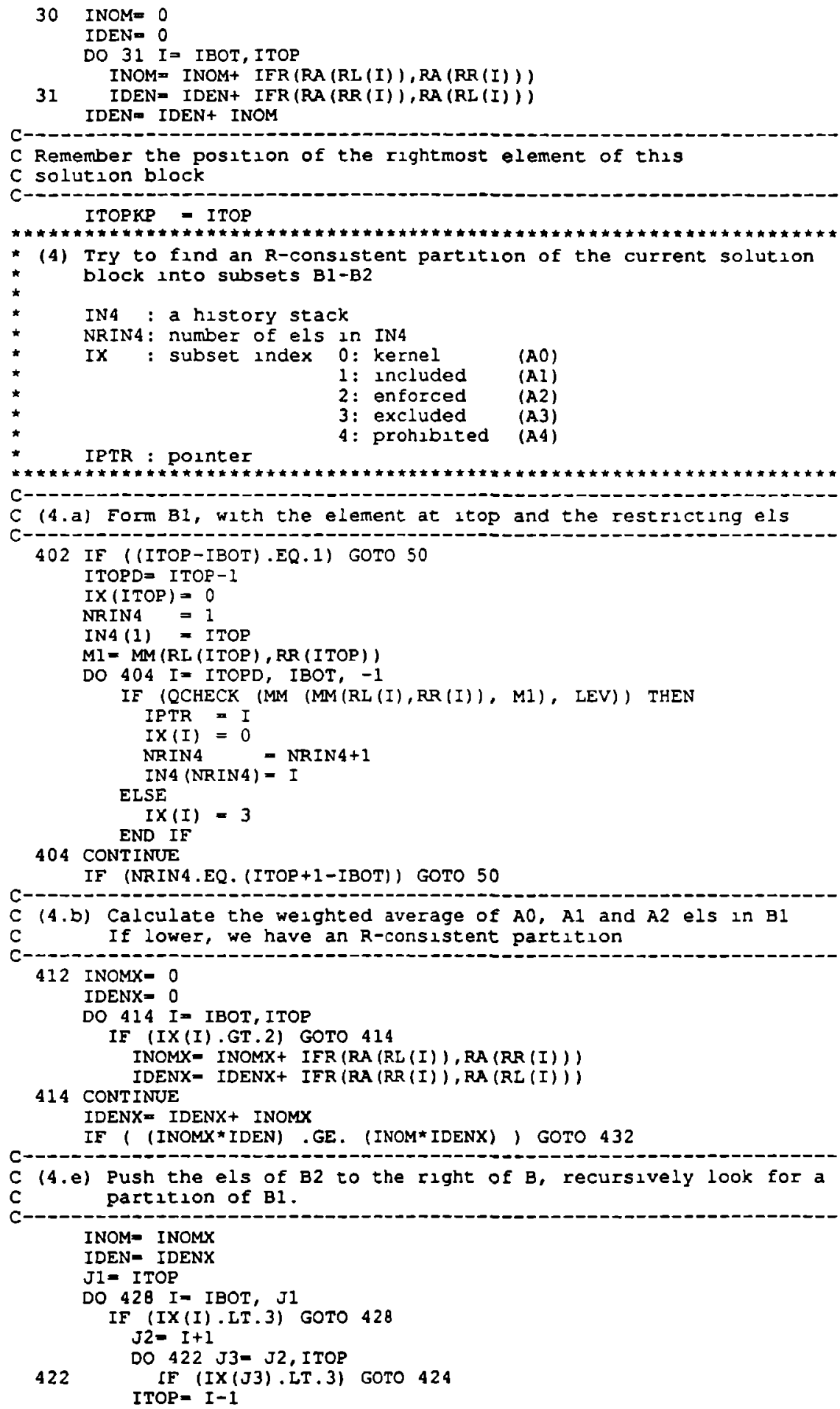




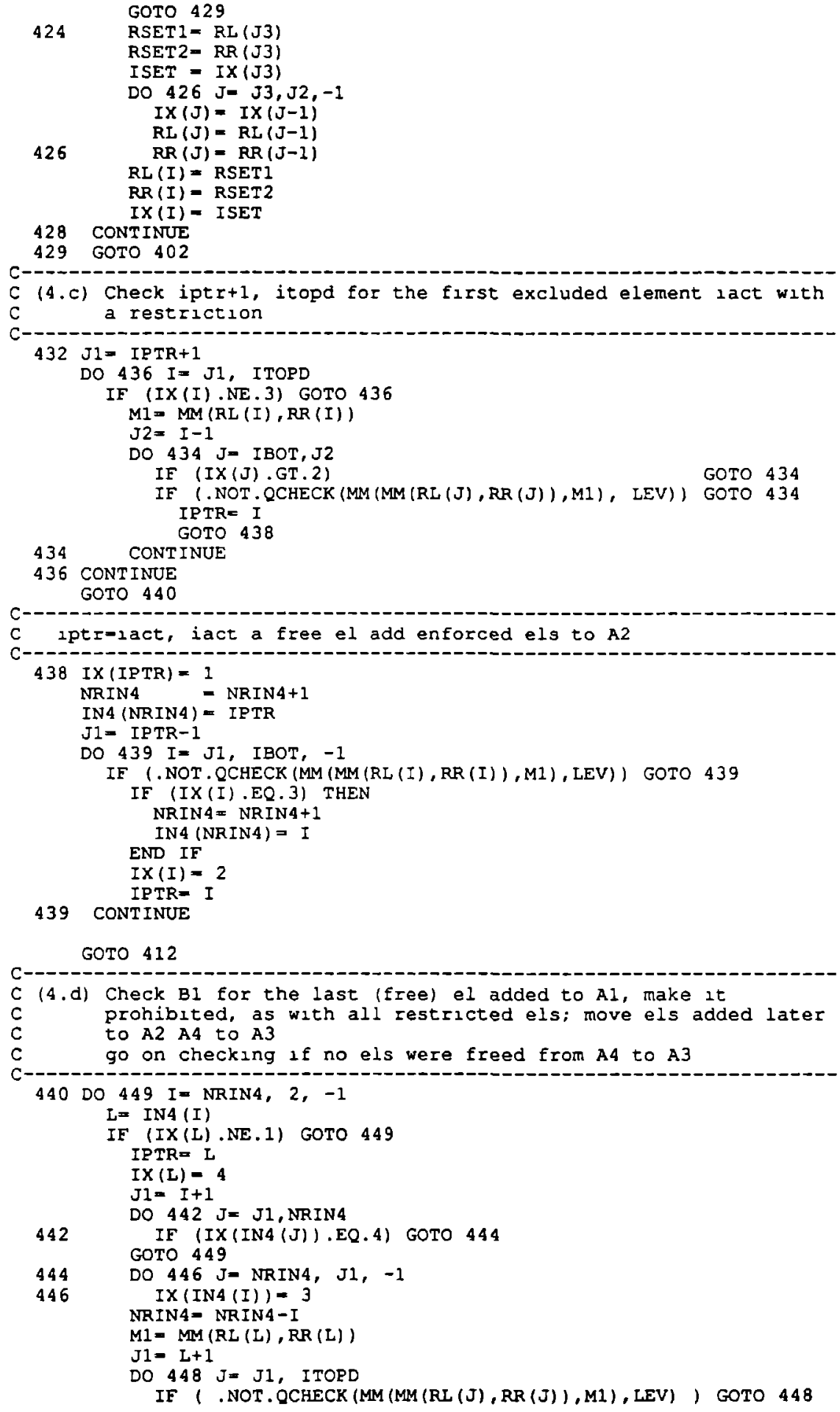




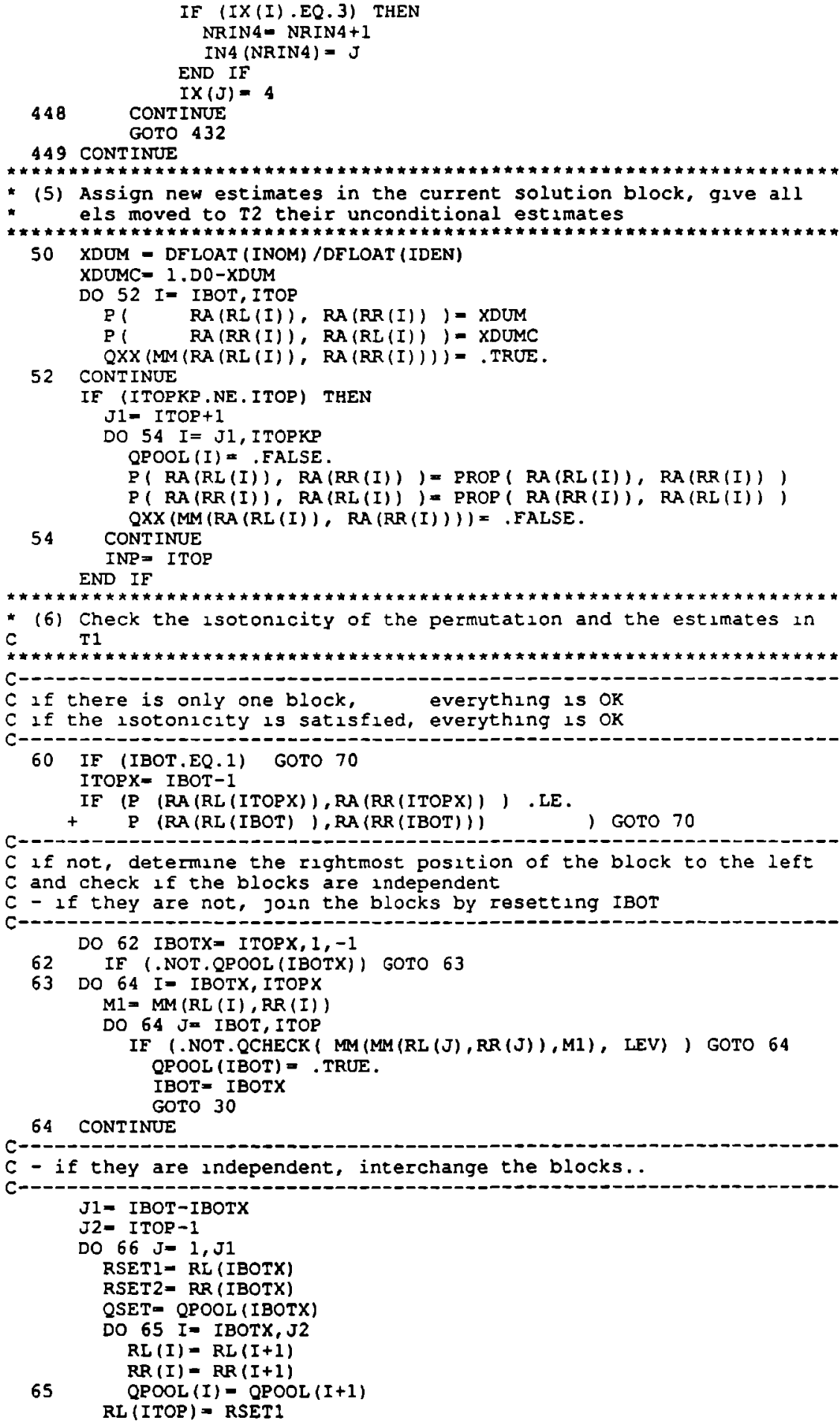




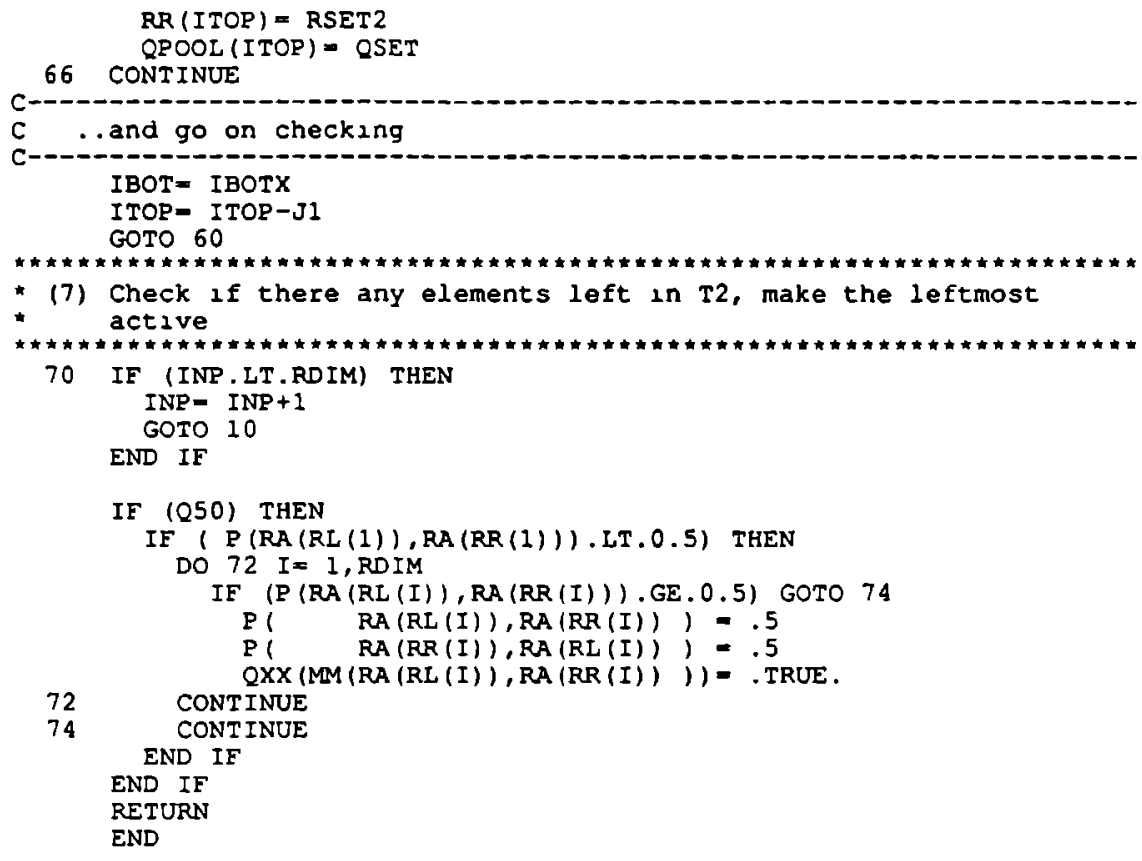

SUBROUTINE BRANCH (NRA, LEV, RA, *)

C
C
C
C
C
C
C
C
C
C
C
C
C
C
C
C
C
C
C
C
C
C
C
C
C
C
C
C
C
C

Function

Branching routine - generates all branches of permutation tree This subroutine is called to branch to the next node at level LEV, thereby generating a new subset of the set of all permuations of the NRA elements.

What happens depends on the circumstances. The algorithm backtracks to the node LEV-1. If there is a branch left that has not been previously explored, the algorithm advances along this branch to a new node at level LEV. The new subset of permutations share the LEV-1 leftmost elements with the previous one. If all branches to level LEV from this node have been examined, the algorithm backtracks along the path to the node at level LEV-2, and checks if all branches have been examined. If, finally, the algorithm backtracks to level 1 and all branches have been examined, the search 15 finished (RETURN 1).

At all times, the vector RA contains a permutation of the NRA elements, where RA(1) contains the index of the element at the 1'th position in the permutation.

Before a permutation tree can be explored, the vector KA has to hold the number of branches from each node. Actually KA(1) contains the value (NRA+I - number of branches from each node at level 1). This is achived by making a initial call to INIBRA.

When a call to BRANCH is made, the algorithm examines the value of KA(1nd). If this value doesn't equals NRA, the next branch is examined. This is achieved by incrementing KA(1nd), and reversing the elements $R A(1 n d)$ and $R A(K A$ (Ind)). If the value of KA (1nd) equals NRA. 


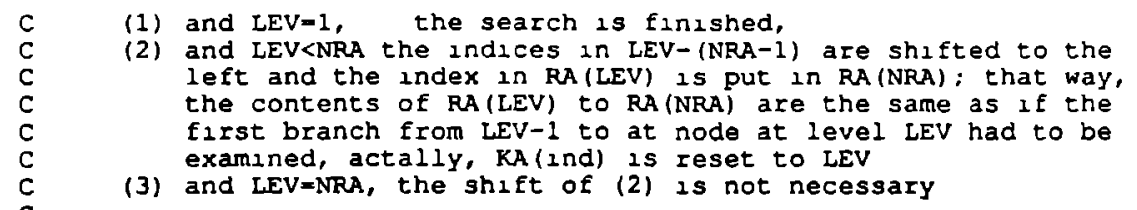

calls none

Parameters

LEV . . . . . . . . current level in permutation tree

NRA . . . . . . . . number of alternatives in tree

RA. . . . . . . . . vector with current rankıng

Local variables

RSET1 . . . . . . . dumny used in interchanging

$\mathrm{C}$

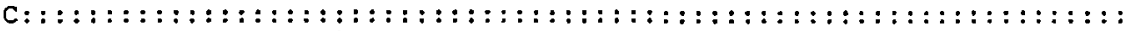

IMPLICIT REAL $* \theta \quad(P, X)$

IMPLICIT INTEGER $(I-N, R)$

IMPLICIT LOGICAL (Q)

PARAMETER $(M X A=13, M X P=(M X A *(M X A-1)) / 2)$

INTEGER RA (MXA)

COMMON / TREE / KA (MXP)

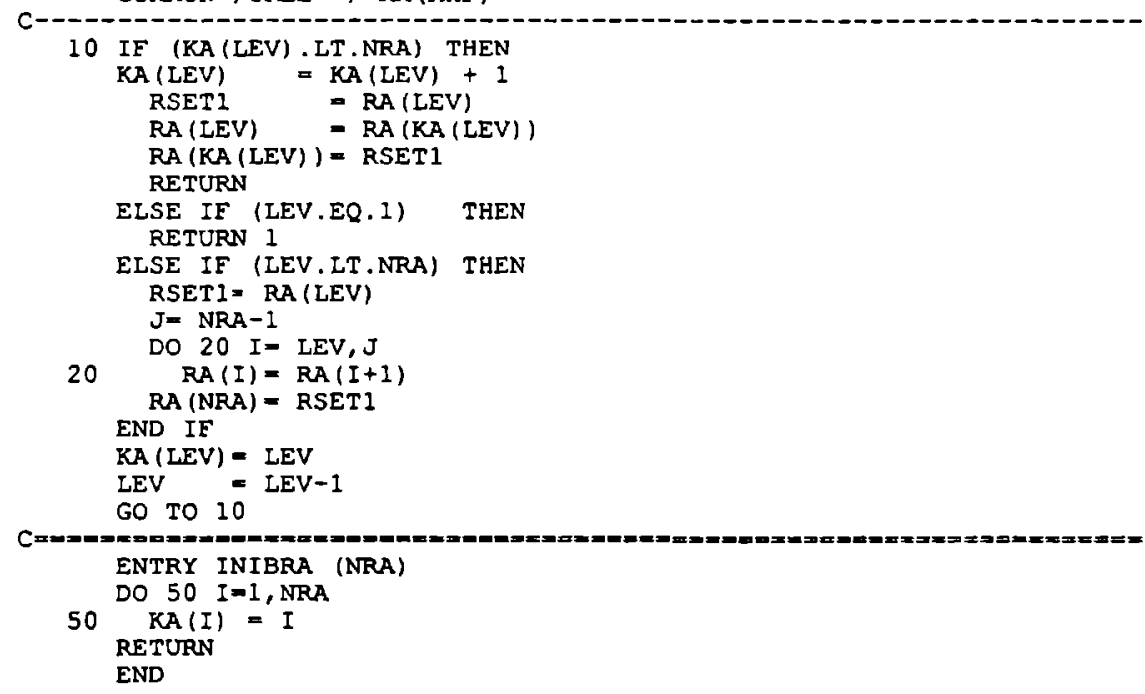




\section{Appendix B \\ Choice proportions}

Table B.1

Choice proportions obtained in experiment 1, task TR4.

\begin{tabular}{lllllllllll}
\hline & $g_{1}$ & $g_{2}$ & $g_{3}$ & $g_{4}$ & $g_{5}$ & $g_{6}$ & $g_{7}$ & $g_{8}$ & $g_{3}$ & $g_{10}$ \\
$g_{1}$ & .500 & .068 & .052 & .119 & .068 & .407 & .276 & .328 & .508 & .576 \\
$g_{2}$ & .932 & .500 & .000 & .456 & .241 & .305 & .475 & .458 & .593 & .690 \\
$g_{3}$ & .948 & 1.000 & .500 & .190 & .237 & .305 & .724 & .661 & .746 & .814 \\
$g_{4}$ & .881 & .544 & .810 & .500 & .576 & .793 & .814 & .966 & .949 & .948 \\
$g_{5}$ & .932 & .759 & .763 & .424 & .500 & .797 & .898 & .932 & .983 & 1.000 \\
$g_{6}$ & .593 & .695 & .695 & .207 & .203 & .500 & .931 & .949 & .949 & .966 \\
$g_{7}$ & .724 & .525 & .276 & .186 & .102 & .069 & .500 & .915 & .949 & 1.000 \\
$g_{3}$ & .672 & .542 & .339 & .034 & .068 & .051 & .085 & .500 & .948 & .915 \\
$g_{9}$ & .492 & .407 & .254 & .051 & .017 & .051 & .051 & .052 & .500 & .915 \\
$g_{10}$ & .424 & .310 & .186 & .052 & .000 & .034 & .000 & .085 & .085 & .500 \\
\hline
\end{tabular}

Table B.2

Choice proportions obtained in experiment 1 , task TR5.

\begin{tabular}{lllllllllll}
\hline & $g_{1}$ & $g_{2}$ & $g_{3}$ & $g_{4}$ & $g_{5}$ & $g_{6}$ & $g_{7}$ & $g_{3}$ & $g_{3}$ & $g_{10}$ \\
$g_{1}$ & .500 & .119 & .034 & .103 & .085 & .068 & .288 & .224 & .407 & .322 \\
$g_{2}$ & .881 & .500 & .086 & .034 & .085 & .069 & .259 & .390 & .362 & .491 \\
$g_{3}$ & .966 & .914 & .500 & .085 & .155 & .169 & .390 & .458 & .789 & .729 \\
$g_{4}$ & .897 & .966 & .915 & .500 & .254 & .339 & .500 & .672 & .831 & .847 \\
$g_{5}$ & .915 & .915 & .845 & .746 & .500 & .707 & .966 & .864 & .897 & .931 \\
$g_{6}$ & .932 & .931 & .831 & .661 & .293 & .500 & .847 & .914 & .914 & .947 \\
$g_{7}$ & .712 & .741 & .610 & .500 & .034 & .153 & .500 & .862 & .949 & .931 \\
$g_{3}$ & .776 & .610 & .542 & .328 & .136 & .086 & .138 & .500 & .949 & .966 \\
$g_{3}$ & .593 & .638 & .211 & .169 & .103 & .086 & .051 & .051 & .500 & .915 \\
$g_{10}$ & .678 & .509 & .271 & .153 & .069 & .053 & .069 & .034 & .085 & .500 \\
\hline
\end{tabular}


Table B.3

Choice proportions obtained in experiment 2, task STG.

\begin{tabular}{lllllllllll}
\hline & $g_{1}$ & $g_{2}$ & $g_{3}$ & $g_{4}$ & $g_{5}$ & $g_{6}$ & $g_{7}$ & $g_{3}$ & $g_{3}$ & $g_{10}$ \\
$g_{1}$ & .500 & .090 & .094 & .104 & .119 & .060 & .182 & .164 & .194 & .197 \\
$g_{2}$ & .910 & .500 & .091 & .288 & .109 & .182 & .194 & .224 & .167 & .234 \\
$g_{3}$ & .906 & .909 & .500 & .104 & .134 & .149 & .269 & .246 & .323 & .379 \\
$g_{4}$ & .896 & .712 & .896 & .500 & .212 & .284 & .273 & .343 & .373 & .409 \\
$g_{5}$ & .881 & .891 & .866 & .788 & .500 & .231 & .269 & .269 & .444 & .455 \\
$g_{6}$ & .940 & .818 & .851 & .716 & .769 & .500 & .313 & .400 & .418 & .515 \\
$g_{7}$ & .818 & .806 & .731 & .727 & .731 & .687 & .500 & .463 & .507 & .642 \\
$g_{3}$ & .836 & .776 & .754 & .657 & .731 & .600 & .537 & .500 & .600 & .642 \\
$g_{9}$ & .806 & .833 & .677 & .627 & .556 & .582 & .493 & .400 & .500 & .567 \\
$g_{10}$ & .803 & .766 & .621 & .591 & .545 & .485 & .358 & .358 & .433 & .500 \\
\hline
\end{tabular}

Table B.4

Choice proportions obtained in experiment 2, task N46.

\begin{tabular}{rrrllllllll}
\hline & 08 & 14 & 27 & 31 & 46 & 59 & 62 & 70 & 85 & 93 \\
08 & .500 & .000 & .000 & .000 & .000 & .024 & .012 & .133 & .341 & .500 \\
14 & 1.000 & .500 & .000 & .012 & .000 & .024 & .036 & .202 & .548 & .614 \\
27 & 1.000 & 1.000 & .500 & .012 & .012 & .094 & .205 & .476 & .690 & .817 \\
31 & 1.000 & .988 & .988 & .500 & .013 & .145 & .470 & .542 & .687 & .902 \\
46 & 1.000 & 1.000 & .988 & .988 & .500 & .602 & .598 & .675 & 1.000 & .988 \\
59 & .976 & .976 & .906 & .855 & .398 & .500 & 1.000 & .988 & .975 & 1.000 \\
62 & .988 & .964 & .795 & .530 & .402 & .000 & .500 & .988 & .988 & .988 \\
70 & .867 & .798 & .524 & .458 & .325 & .012 & .012 & .500 & .988 & .988 \\
85 & .659 & .452 & .310 & .313 & .000 & .025 & .012 & .012 & .500 & .976 \\
93 & .500 & .386 & .183 & .098 & .012 & .000 & .012 & .012 & .024 & .500 \\
\hline
\end{tabular}




\section{Table B.5}

Choice proportions obtained in experiment 2, task N59.

\begin{tabular}{rrrrlllllll}
\hline & \multicolumn{1}{c}{08} & 14 & 27 & 31 & 46 & 59 & 62 & 70 & 85 & 93 \\
08 & .500 & .000 & .012 & .000 & .000 & .000 & .000 & .071 & .217 & .325 \\
14 & 1.000 & .500 & .000 & .024 & .000 & .024 & .024 & .141 & .298 & .446 \\
27 & .988 & 1.000 & .500 & .012 & .000 & .083 & .193 & .366 & .553 & .753 \\
31 & 1.000 & .976 & .988 & .500 & .012 & .159 & .333 & .349 & .634 & .841 \\
46 & 1.000 & 1.000 & 1.000 & .988 & .500 & .381 & .417 & .500 & .976 & .988 \\
59 & 1.000 & .976 & .917 & .841 & .619 & .500 & .988 & .976 & 1.000 & 1.000 \\
62 & 1.000 & .976 & .807 & .667 & .583 & .012 & .500 & 1.000 & .965 & .988 \\
70 & .929 & .859 & .634 & .651 & .500 & .024 & .000 & .500 & .976 & 1.000 \\
85 & .783 & .702 & .447 & .366 & .024 & .000 & .035 & .024 & .500 & 1.000 \\
93 & .675 & .554 & .247 & .159 & .012 & .000 & .012 & .000 & .000 & .500 \\
\hline
\end{tabular}

Table B.6

Choice proportions obtained in experiment 2, task PP.

EVP CPN PSP PPR PVdA D66 CDA VVD RPF GPV SGP $\begin{array}{llllllllllll}\text { EVP } & .500 & .376 & .224 & .200 & .153 & .176 & .400 & .482 & .659 & .682 & .679\end{array}$ $\begin{array}{llllllllllll}\text { CPN } & .624 & .500 & .165 & .259 & .271 & .459 & .612 & .682 & .753 & .765 & .741\end{array}$ $\begin{array}{llllllllllll}\text { PSP } & .776 & .835 & .500 & .529 & .447 & .576 & .706 & .788 & .882 & .882 & .894\end{array}$ $\begin{array}{llllllllllll}\text { PPR } & .800 & .741 & .471 & .500 & .435 & .619 & .694 & .765 & .859 & .882 & .882\end{array}$ $\begin{array}{llllllllllll}\text { PvdA } & .847 & .729 & .553 & .565 & .500 & .635 & .812 & .824 & .918 & .918 & .941\end{array}$ $\begin{array}{lllllllllllll}D 66 & .824 & .541 & .424 & .381 & .365 & .500 & .800 & .800 & .976 & .988 & .976\end{array}$ $\begin{array}{llllllllllll}C D A & .600 & .388 & .294 & .306 & .188 & .200 & .500 & .682 & .929 & .918 & .953\end{array}$ $\begin{array}{llllllllllll}\text { WD } & .518 & .318 & .212 & .235 & .176 & .200 & .318 & .500 & .835 & .859 & .859\end{array}$ $\begin{array}{llllllllllll}\text { RPF } & .341 & .247 & .118 & .141 & .082 & .024 & .071 & .165 & .500 & .512 & .518\end{array}$ $\begin{array}{llllllllllll}\text { GPV } & .318 & .235 & .118 & .118 & .082 & .012 & .082 & .141 & .488 & .500 & .494\end{array}$ $\begin{array}{llllllllllll}\text { SGP } & .321 & .259 & .106 & .118 & .059 & .024 & .047 & .141 & .482 & .506 & .500\end{array}$ 
Table B.7

Choice proportions obtained in experiment 2, task CCl (petty extortion).

\begin{tabular}{lccccccc}
\hline & 2 & 4 & 6 & 9 & 12 & 15 & 18 \\
2 & .500 & .106 & .294 & .412 & .588 & .647 & .718 \\
4 & .894 & .500 & .424 & .541 & .647 & .753 & .871 \\
6 & .706 & .576 & .500 & .659 & .800 & .869 & .812 \\
9 & .588 & .459 & .341 & .500 & .859 & .871 & .941 \\
12 & .412 & .353 & .200 & .141 & .500 & .918 & .988 \\
15 & .353 & .247 & .131 & .129 & .082 & .500 & .988 \\
18 & .282 & .129 & .188 & .059 & .012 & .012 & .500 \\
\hline
\end{tabular}

Table B.8

Choice proportions obtained in experiment 2, task CC2 (counterfeiting).

\begin{tabular}{lccccccc}
\hline & 2 & 4 & 6 & 9 & 12 & 15 & 18 \\
2 & .500 & .118 & .212 & .376 & .471 & .541 & .659 \\
4 & .882 & .500 & .329 & .506 & .565 & .718 & .765 \\
6 & .788 & .671 & .500 & .612 & .682 & .718 & .800 \\
9 & .624 & .494 & .388 & .500 & .788 & .871 & .871 \\
12 & .529 & .435 & .318 & .212 & .500 & .882 & .894 \\
15 & .459 & .282 & .282 & .129 & .118 & .500 & .965 \\
18 & .341 & .235 & .200 & .129 & .106 & .035 & .500 \\
\hline
\end{tabular}

\section{Table B.9}

Choice proportions obtained in experiment 2, task CC3 (intentional endangering of traffic).

\begin{tabular}{lccccccc}
\hline & 2 & 4 & 6 & 9 & 12 & 15 & 18 \\
2 & .500 & .353 & .482 & .694 & .741 & .812 & .882 \\
4 & .647 & .500 & .647 & .788 & .894 & .859 & .918 \\
6 & .518 & .353 & .500 & .835 & .929 & .894 & .976 \\
9 & .306 & .212 & .165 & .500 & .965 & .965 & 1.000 \\
12 & .259 & .106 & .071 & .035 & .500 & .976 & .988 \\
15 & .188 & .141 & .106 & .035 & .024 & .500 & .976 \\
18 & .118 & .082 & .024 & .000 & .012 & .024 & .500 \\
\hline
\end{tabular}



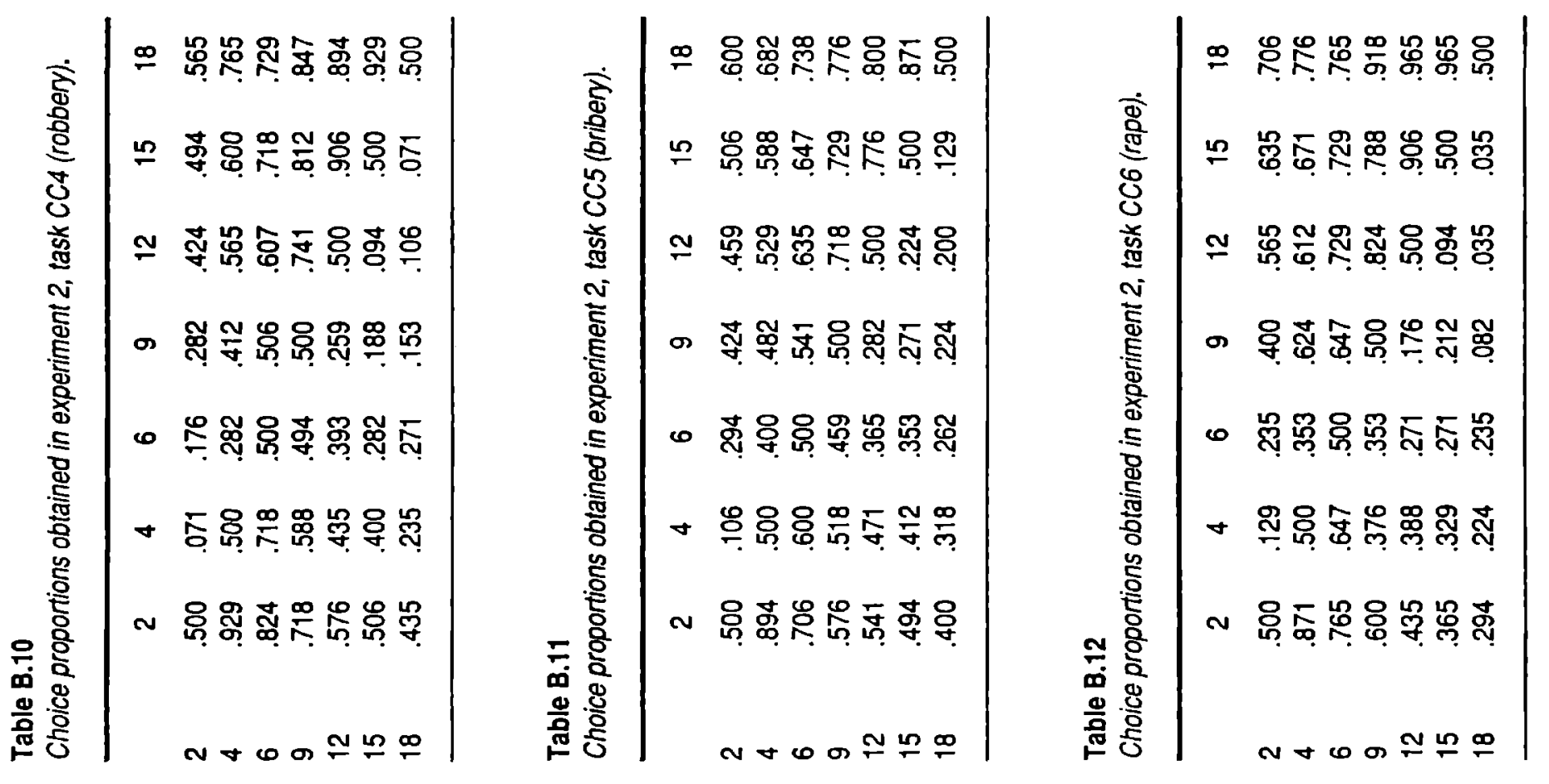



\section{Appendix C Distributions}

Each table contains estimates of quantiles of the distributions of the test statistic minus $2 \mathrm{log}$ likelihood ratio if the respective condition is satisfied, for combinations of number of altematives $\left(\kappa_{x}\right)$ and number of presentations $\left(n_{x y}\right)$. Each set of quantiles was estimated nonparametrically in a series of 250 Monte Carlo simulations, using order statistics.

In Experiment 1, there were 59 presentations $\left(n_{x y}\right)$ of all pairs out of 10 alternatives $\left(\kappa_{x}\right)$ in tasks TR4 and TR5, and 67 presentations of all pairs out of 10 altematives in tasks STG. In Experiment 2, there were 85 presentations of all pairs out of 7 alternatives in the Criminal Code tasks CC1-CC6, of all pairs out of 10 altematives in the "numbers" tasks N46 and N59, and of all pairs out of 11 altematives in the "political parties" task PP.

Table C.1

Weak stochastic ranking (STA).

\begin{tabular}{ccccccc}
\hline \multirow{2}{*}{$\kappa_{x}$} & $n_{x y}$ & \multicolumn{5}{c}{ estimated quantiles } \\
\cline { 3 - 7 } & & .50 & .75 & .90 & .95 & .99 \\
\hline \multirow{2}{*}{7} & 85 & .00 & .29 & 1.44 & 2.67 & 5.83 \\
10 & 59 & .42 & 1.41 & 2.91 & 3.86 & 6.79 \\
& 67 & .39 & 1.59 & 2.97 & 4.38 & 9.54 \\
& 85 & .31 & 1.43 & 2.70 & 4.21 & 7.83 \\
11 & 85 & .58 & 1.55 & 3.55 & 4.91 & 6.78 \\
\hline
\end{tabular}




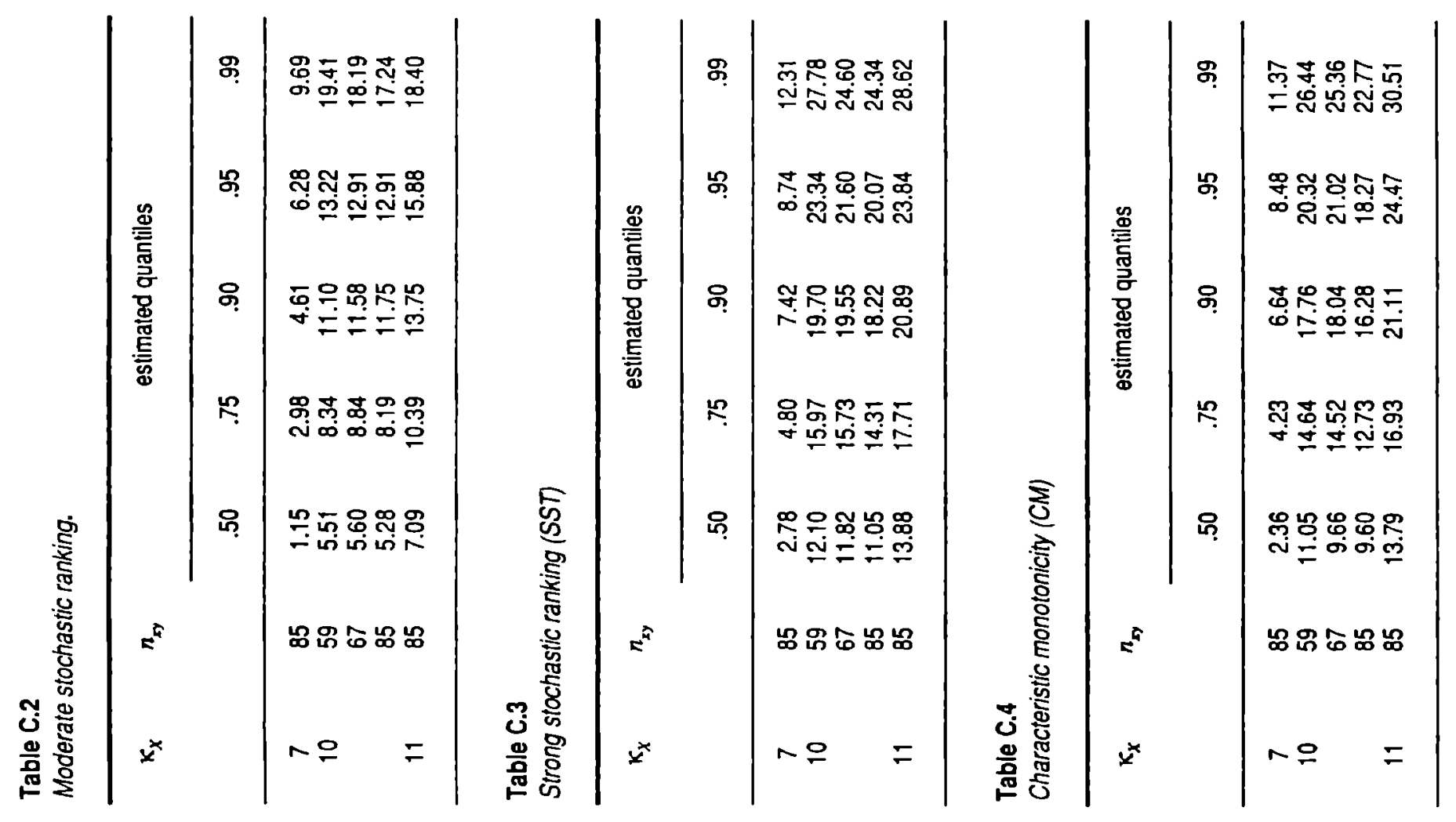


Table C.5

Bilateral monotonicity (BM)

\begin{tabular}{|c|c|c|c|c|c|c|}
\hline \multirow[t]{2}{*}{$x_{x}$} & \multirow[t]{2}{*}{$n_{x y}$} & \multicolumn{5}{|c|}{ estimated quantiles } \\
\hline & & .50 & .75 & .90 & .95 & .99 \\
\hline $\begin{array}{c}7 \\
10\end{array}$ & $\begin{array}{l}85 \\
59 \\
67 \\
85 \\
85\end{array}$ & $\begin{array}{r}.83 \\
6.44 \\
6.08 \\
5.43 \\
8.78\end{array}$ & $\begin{array}{r}2.01 \\
8.54 \\
9.52 \\
8.44 \\
11.88\end{array}$ & $\begin{array}{r}3.45 \\
11.20 \\
12.50 \\
11.33 \\
16.29\end{array}$ & $\begin{array}{r}5.04 \\
13.64 \\
15.31 \\
12.90 \\
17.93\end{array}$ & $\begin{array}{r}8.20 \\
21.49 \\
21.99 \\
18.59 \\
21.78\end{array}$ \\
\hline
\end{tabular}

Table C. 6

Bilateral/charateristic monotonicity (BCM)

\begin{tabular}{ccrrrrr}
\hline \multirow{2}{*}{$\kappa_{x}$} & $n_{x y}$ & \multicolumn{5}{c}{ estimated quantiles } \\
\cline { 3 - 7 } & & \multicolumn{6}{c}{.50} & \multicolumn{1}{c}{.75} & .90 & .95 & .99 \\
\hline 7 & 85 & .94 & 1.88 & 3.75 & 4.65 & 5.71 \\
10 & 59 & 7.75 & 10.51 & 12.96 & 14.26 & 17.05 \\
& 67 & 6.48 & 9.54 & 12.89 & 16.63 & 23.40 \\
& 85 & 6.33 & 10.17 & 11.99 & 13.41 & 15.84 \\
11 & 85 & 9.25 & 12.63 & 16.97 & 19.36 & 20.94 \\
\hline
\end{tabular}

\section{Table C.7}

Ideal point condition IP.2

\begin{tabular}{ccrrrrr}
\hline \multirow{2}{*}{$\kappa_{x}$} & $n_{x y}$ & \multicolumn{5}{c}{ estimated quantiles } \\
\cline { 3 - 7 } & & .50 & .75 & .90 & .95 & .99 \\
\cline { 3 - 7 } & & & & & \\
\hline & & & & \\
10 & 85 & .55 & 1.51 & 2.84 & 4.21 & 6.78 \\
& 59 & 2.29 & 4.23 & 6.35 & 7.69 & 11.45 \\
& 67 & 1.90 & 3.62 & 6.26 & 8.16 & 12.47 \\
11 & 85 & 1.89 & 3.52 & 5.76 & 7.09 & 12.03 \\
& 85 & 2.67 & 4.81 & 7.19 & 9.08 & 11.08 \\
\hline
\end{tabular}





\section{List of Symbols}

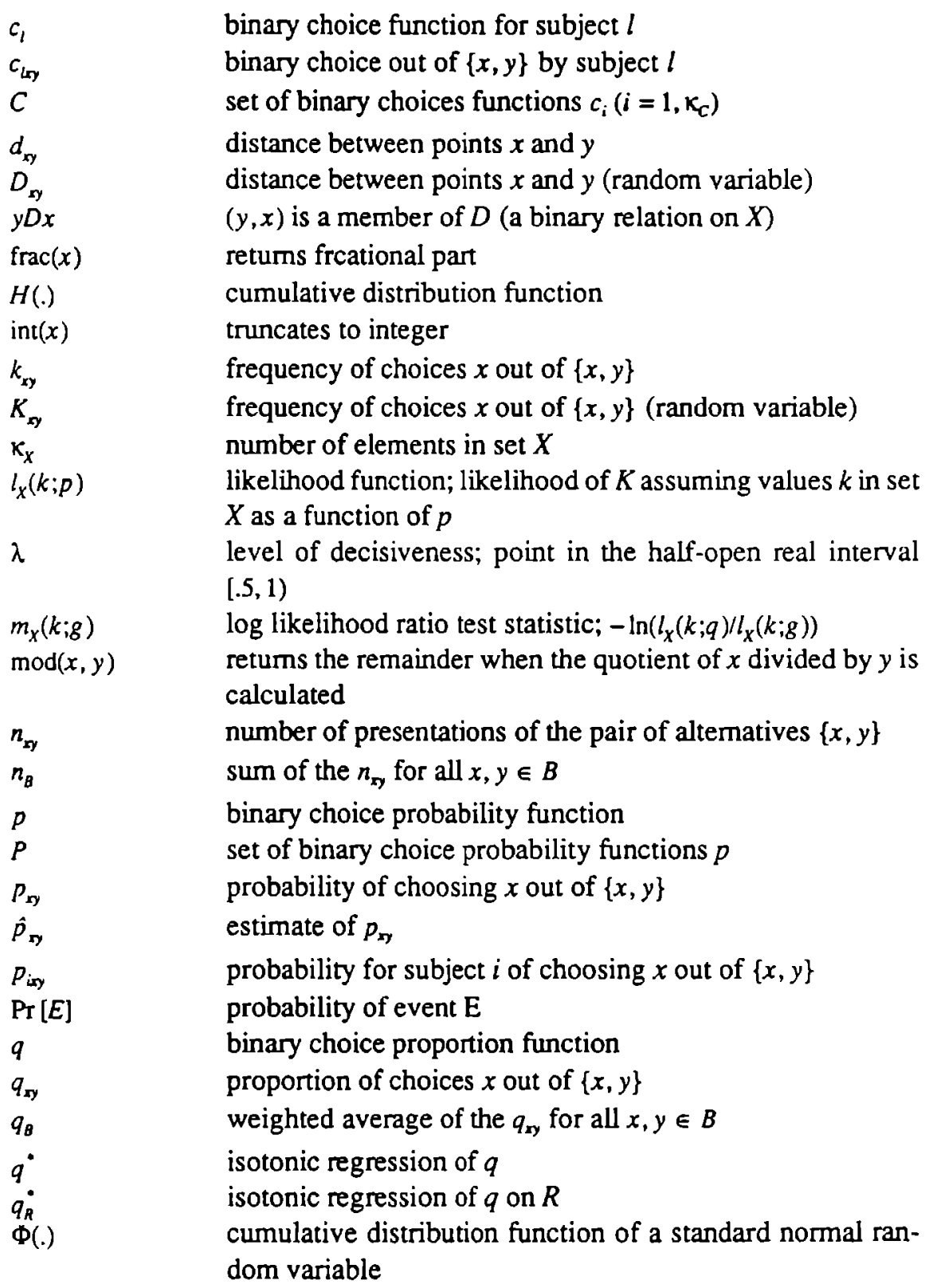




\begin{tabular}{|c|c|}
\hline$r(X)$ & ranking of the elements in $X$ \\
\hline$R(X)$ & set of all possible rankings of the elements in $X$ \\
\hline$\rho_{r_{u} r_{r}}$ & correlation coefficient of random variables $T_{\mathrm{xx}}$ and $T_{x y}$ \\
\hline$\sigma_{x}^{2}$ & variance of the random variable $X$ \\
\hline$\sigma_{z}^{2}$ & variance of the random variable $Z$ \\
\hline$t_{u}$ & difference between values of the coordinates of $z$ and $x$ \\
\hline$T_{2 x}$ & $\begin{array}{l}\text { difference between values of the coordinates of } z \text { and } x \\
\text { (random variable) }\end{array}$ \\
\hline$\theta()$. & $\begin{array}{l}\text { function, retums }-1 \text { if argument is negative, } 1 \text { if argument is } \\
\text { positive, } 0 \text { otherwise }\end{array}$ \\
\hline$\Theta$ & parameter space, set of possibles value of $p$ \\
\hline$\Theta^{I P 4}$ & subset of $\theta$ in which the values satisfy condition IP.4 \\
\hline$\Theta_{a(X)}^{S T A}$ & $\begin{array}{l}\text { subset of } \Theta \text { in which STA is satisfied, conditional on the } \\
\text { ranking } r(X) \text { known to be } D \text {-isotonic }\end{array}$ \\
\hline$u_{z}(x)$ & $\begin{array}{l}\text { preference (utility) of alternative } x \text { for a subject with } z \text { as } \\
\text { ideal altemative (in Fechnerian strong utility model) }\end{array}$ \\
\hline $\overrightarrow{w x y}$ & $x$ precedes $w$ and is preceded by $y$ in a ranking \\
\hline$X$ & set of alternatives \\
\hline$x$ & alternative (coordinate of the alternative $x$ ) \\
\hline $\mathbf{x}$ & coordinate vector of the altemative $x$ \\
\hline $\mathbf{X}$ & coordinate vector of the altemative $x$ (random variable) \\
\hline$y$ & altemative (coordinate of the altemative $y$ ) \\
\hline$z$ & ideal alternative (coordinate of the ideal alternative) \\
\hline$Z$ & coordinate of the ideal altemative (random variable) \\
\hline$z_{1}$ & $\begin{array}{l}\text { coordinate of the ideal alternative used in calculating the } \\
\text { distance to the first alternative (random variable) }\end{array}$ \\
\hline$Z_{2}$ & $\begin{array}{l}\text { coordinate of the ideal altemative used in calculating the } \\
\text { distance to the second altemative (random variable) }\end{array}$ \\
\hline $\mathbf{z}$ & coordinate vector of the ideal alternative $z$ \\
\hline $\mathbf{Z}$ & coordinate vector of the ideal altemative $z$ (random variable) \\
\hline $\begin{array}{l}\zeta_{q} \\
\neg(.)\end{array}$ & $\begin{array}{l}\text { qth quantile of the distribution of a random variable } \\
\text { logical not }\end{array}$ \\
\hline$\wedge$ & logical and \\
\hline$\vee$ & logical or \\
\hline 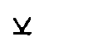 & logical exclusive or \\
\hline
\end{tabular}




\section{Author Index}

Ahrens, J.H., 112

Akaike, H., 73

Andrich, D., 8, 18, 20, 23

Avrunin, G.S., 19

Ayer, M., 83

Barlow, R.E., 83, 109, 110

Bartholomew, D.J., 83, 109, 110

Beals, R., 19

Bechtel, G.G., 6, 8, 9, 15, 18, 20, 23, 26, 68

Benneth, J.F., 1

Bezembinder, Th., 43, 108

Block, H.D., 8, 17, 58

Bock, R.D., 4, 66

Bossuyt, P.M.M., 8, 29, 43, 53, 107, 119,140

Box, B.E.P., 66

Bozdogan, H., 2

Bradley, R.A., 1

Bremner, J.M., 83, 109, 110

Brunk, H.D., 83, 109, 110

Bulgren, W.G., 13

Candel, M., 140

Carroll, J.D., 2, 6, 14, 20, 23, 26

Chipman, J.S., 27, 28

Coombs, Cl.H., 4, 5, 6, 7, 10, 11, 12, 14, $15,18,19,22,27,28,29,30,38,40$. $59,60,61,67,104,119,121,122$. $126,127,128,136,140,141$

Cooper, L.G., 8, 18, 20, 23

Croon, M., 3, 7, 15, 16, 23, 29, 32, 34, $45,48,49,69,70$

David, H.A., 1,66

Davidson, D., 8
Davison, M., 20

De Leeuw, J., 2

De Soete, G., 2, 6, 7, 14, 17, 23, 26, 29

Debreu, 9

DeCani, J.S., 100

DeSarbo, W.S., 2, 6, 7, 14, 17, 23, 26, $29,32,49$

Dieter, U., 112

Dijkstra, L., 37

Dykstra, R.L., 83, 84, 97, 98, 109, 110

Edgell, S.E., 118

Eisler, H., 7, 17, 23

Eliashberg, J., 17, 23, 29

Ewing, G.M., 83

Falmagne, J.C., 8

Fechner, G.F., 1, 4, 139

Fishburn, P.C., 27, 30

Flueck, J.A., 99, 100, 106

Fumas, G.W., 2

Gaul, W., 6, 14, 23

Gebhardt, F., 86, 93, 94, 95

Geisler, W.S., 118

Georgescu-Roegen, N., 27

Greenberg, M.G., 5, 6, 15, 68, 104, 105 , 119,120

Greeno, J.G., 28, 116, 117, 118

Griggs, R.A., 5, 6, 7, 9, 10, 11, 12, 13 , $14,15,23,29,38,39,40,43,44,45$, $46,47,48,51,68,69,70,127,128$

Gumbel, EJ., 16

Halff, H.M., 9, 21, 42

Hays, W.L., 1

Hefner, R.A., 13 
Heiser, W.J., 2, 139

Huber, L., 100

Hunter, W.G., 66

Hunter, J.S., 66

Indow, T., 69

Jansen, P.G.W., 8, 9, 18, 19, 23

Jedidi, K., 26

Jones, L.V., 4, 66

Kendall, M.G., 108

Korsh, J.F., 99, 100, 106

Krantz, D.M., 18, 19, 28, 41, 55, 58, 115,127

Kruskal, J.B., 2, 83, 86

Luce, R.D., 3, 8, 17, 18, 19, 22, 24, 26, $30,55,58,59,63,64,66,67,118$. 139

Marschak, J., 8, 17, 26, 27, 58

May, K.O., 28

Molenaar, I.W., 37

Morrison, H.W., 28

Nakanishi, M., 8, 18, 20, 23

Patnaik, P.B., 12

Payne, C.D., 108

Phillips, J.P.N., 108

Ramsay, J.O., 6, 7, 9, 13, 15, 16, 23, 29 , $32,34,35,45,48,49,69,70$

Ranyard, R.H., 100, 108

Reid, W.T., 83

Remage, R., 100

Restle, F., 118, 139

Rice, W.R., 113

Roberts, F.S., 27

Robertson, T., 83, 84, 109, 110
Roskam, E.E.Ch.I., 2, 8, 53, 107, 119 , 140

Ross, R.T., 123

Rumelhart, D.L., 28, 116, 117, 118

Russo, J.E., 27, 28, 41, 56

Schönemann, P.H., 2, 8, 18, 19, 20, 23

Schultz, J., 100

Shepard, R.N., 2, 18

Shibayama, T., 2

Shocker, A.D., 20

Silverman, E., 83

Singh, J., 100

Sixtl, F., 9, 23

Slater, P., 108

Smith, A.F.M., 108

Srinivasan, V., 20

Stokman, F.N., 37

Suppes, P., 3, 8, 17, 22, 24, 26, 55, 58, $59,60,63,64,66,67$

Takane, Y., 2, 69

Thompson, W.A., 100

Thurstone, L.L., 4, 5, 6, 7, 8, 118, 138 , 139

Tversky, A., 19, 27, 28, 41, 55, 56, 58, $115,116,117,139$

Valvanis-Vail, S., 27

Van der Eijk, C., 37

Van Eeden, C., 83

Van Schuur, W.H., 2, 37, 128

Verhelst, N., 37

Wang, W.M., 8, 18, 19, 20, 23

Wright, F.T., 83, 84, 109, 110

Zinnes, J.L., 5, 6, 7, 9, 10, 11, 12, 13 , $14,15,23,29,38,39,40,43,44,45$. $46,47,48,51,58,68,69,70,118$, 127,128 


\section{Subject Index}

Acoustics, 1

AIC, 73

Amsterdam expenment, 121

Animal ecology, 1

BCM

See monotoncity, bilateral/charactenstic

BCP structure

See binary choice probability, structure

BCP system

See binary choice probability, system

Bernoull trial, 3, 55, 61, 66, 67

Bilateral condition, 35, 36, 104, 105, $124,125,126,127,132,133,135$

Binary choice frequency structure, 96, 104

Binary chorce probability structure, $10,17,24,25,27,29,41$, $42,43,45,46,47,48,51,57,58$, $59,61,62,63,64,66,75,76,80$, $81,82,107,118,119$ system, 10, 15, 17, 18, 21, 25, 31, 51, $75,96,107$

BM

See monotonicity, bilateral

Branch and bound, 64, 100

Branch search, 73, 74, 98, 100, 101, $102,104,106,107,115,140$

Choice behavior, $1,8,9,21,25,26,29$, $30,31,36,53,59,60,63,65,70,73$, $115,117,119,121,124,126,128$. $129,137,138,140,141$

Choice vanability, $3,5,6,9,31$, I28, 129,138
CM

See monotonicity, characteristic

Concave, $21,32,33,34,42,49,50$

Convex, 32, 33, 35, 49, 50, 82, 85

Coombs-Zinnes-Gnggs approach, 7, 10,

$11,14,15,29,38,40,127,128$

Case I, 12, 15, 23, 28, 32, 38, 39, 43, $45,48,51$

Case II, 13, 23, 28, 32, 38, 39, 40, 48. 128

Case III, 14, 23, 28, 38, 39, 45, 46, 48, 51,128

Case IV, 15, 23, 28, 32, 39, 43, 45, 48, 51

Critical region, 108, 111, 112, 113, 115, 118,119

Data-representation, 1

Dentustry, 1

Discriminal process, 4, 8, 138

Discriminant analysis, 2

Dissumilantes, $1,2,3,6,7,9,15,18$, $21,22,25,67,69,140$

Distribution extreme value, 16 logistic, $16,18,43$ lognomal, 7, 16 noncentral chi-square, $11,12,13$ (doubly) noncentral $F, 12,13$ normal, 5.6, 7, 11, 13, 15, 17, 34, 35, $37,68,108,110,111$

Weibull, 7, 16

Dominance matrix, 37, 39, 40

Economics, 1

Epidemiology, 1 
Enor distribution, 7, 16, 32, 33, 34

Fechnenan scaling, 8, 18

Food science, 1

Goodness-of-fit, 20, 62, 63, 64, 65, 70, 73,140

Grayness, 123, 138

Ideal, $36,37,38,39,40,43,46,48,49$, $68,69,70,71,76,77,78,96,104$, $105,122,124,126,127,128,129$, $132,133,134,135,136,137,138$, $139,140,141$

wandenng, 6, 14

Ideal point condition

IP $1,30,32,47,48,76,96,122,124$, $125,126,127,133,134,138$

IP 2, 30, 31, 32, 47, 48, 76, 96, 124, $125,126,127,133,134,135,137$, 138

IP 3, 31, 32, 33, 35, 48, 49, 70, 71, 75, $122,124,125,126,127,134,137$. 138

IP 4, 31, 32, 33, 48, 49, 50, 51, 76, 122, $124,125,126,127,132,133,135$, 138

Inconsistency, 2, 3, 36, 65, 67, 107, 138

Isotonic rankıng, $81,87,89,90,92,94$, $99,106,108,111$

$D$-Isotonic, 79, 80, 81, 99, 103, 106, 107

$q$-Isotonic, 87, 89, 90

$R$-Isotonic, 86, 87, 112

Isotonic regression, $74,83,84,86,87$, $93,95,97,98,113$

$J$ scale, 76

Level of decisiveness, 30,31

Likeluhood ratio, 62, 64, 73, 74, 100 , $101,107,110,112,113,115,117$. $119,125,135,140$ generalızed, $62,64,73,74,100,101$, $107,110,112,115,125,140$
Marketıng, 1

Maximal $R$-consistent partinon, 85, 86, $87,89,90,93,97,108$

Maxımum lukellhood, 81, 82, 83, 86, 97 , $99,100,102,103,104,105,106,107$. $108,110,111,113,114,115,116$, $117,118,119,122,124,125,126$, $133,135,136,138,140$

MDS

See multıdimensional scalıng

Midpoint unfolding model, 21, 28, 29, 32, 33, 38, 39, 46. $47,48,51,68,70,120,128,141$ theory, $9,21,23,24,25,68,70$

Model of data, $55,60,70,73,74,119,129$, 139

of theory, $10,55,57$

specification, $3,5,6,8,9,15,16,23$, $25,68,73,118$

Moderate unfolding model, 21, 42, 46 theory, 9

Moderate utility model, 9 theory, 9, 21

Monotonicity bilateral, 38, 39, 40, 51, 76, 77, 78, 96, $105,107,125,126,127,134,135$ bilateral/charactenstuc, 39, 40, 51, 76, $77,78,96,105,107,112,125,126$, $127,134,135,138$ charactensuc, $36,37,38,39,40,41$, $51,76,77,78,96,104,105,107$. $118,119,125,126,127,134,135$, $136,138,140$ midpoint, 107, 119

Monte Carlo, 110, 111, 112, 113, 116, 124,132

MS rankung

See stochastuc rankang, moderate MST

See stochastuc transitivity, moderate MUDFOLD, 2

Muladımensional scalıng, 2, 6, 7, 13 , $18,20,83$ 
Operations research, 100

Optics, 1

Order statistics, 65, 111, 124, 132

Ordinal restrictions, $39,74,75,76,77$, $82,105,107,140$

Paired comparisons, 1, 3, 4, 7, 10, 15, $22,25,29,55,65,66,67,99,100$, $118,121,122,123,124,126,127$. $128,129,131,134,137$

Parametric assumption, 6, 15

Path length metric, 2

Personnel testing, 1

PF

See preference function

Political parties, 128, 129, 130, 131, $132,135,137,138$

Preference function, $9,17,18,19,20$, $21,32,33,34,35,48,49,50$

single-peaked, 19

Preference relation, 27, 29, 30, 79, 99. 100,140

Preference testing, 1

Probabilistic transitivity

See stochastic transitivity

Probabilistic choice behavior, $8,9,21$, $25,26,29,30,31,36,53,59,63,65$, $70,73,117,119,121,124,126,137$. 140,141

Psychology, 4, 54, 122

Psychometrics, 1

Psychophysical scaling, 20

Quadratic assignment, 100

Quadratic programming, 83

$R$-consistent partition, $85,86,87,89,90$, $91,92,93,94,95,97,108,109$

$R$-constrained elements, 89, 91

Random configuration model, 28, 32

theory, 3, 4, 8, 22, 23, 24, 25, 36

Random coordinate

model, 24, 28, 32, 45, 46, 128, 136 , 141 theory, 4, 5, 6, 8, 9, 13, 22, 23, 24, 32, $34,39,126,127,129,141$

Random distance

model, 7, 15, 16, 17, 24, 28, 29, 32 , $33,34,43,45,48,49,51,127,136$ theory, $4,6,7,9,15,20,22,23,24$. $25,29,34,35,39,68,70$

Random response

model, 24, 28, 32

theory, 3, 8, 9, 22, 23, 24, 25, 69

Random utility theory, 3, 8, 22, 23

Random variability, 3, 4, 5, 6, 32, 36, 129

Random variable, $3,5,6,10,11,12,15$, $22,23,33,38,39,68,69,109,128$

Ranking tree, 99, 100, 101, 103, 104, $105,106,111,115$

Sampling

Double, 5, 12, 15, 22, 23, 28, 29, 32 . $38,39,43,127$

Single, 5, 13, 14, 15, 22, 23, 28, 32 , $38,39,40,69,70,126,128,136$, 141

Sampling scheme, 5, 53, 65, 67, 69, 70 . 71

multiple-judgment $69,70,71,141$

Sensory testing, 1

Seriation, 99

Simple distance decomposability, 41 weak, 41, 43, 47, 51

Simple scalability, 41

Space

Euclidean, 1, 3, 10, 11

psychological, 1, 2, 5, 6, 11, 18, 19,

$26,33,68,76,121,127,128$

unidimensional, $1,3,6,11,12,14,21$, $26,33,35,36,37,50,51,76,96$,

$105,118,121,128,136,137,140$

Spaced order, 66, 122, 123, 131, 132

Sports, 1

ss ranking

See stochastic ranking, strong SST

See stochastic transitivity, strong STA

See stochastic acyclicity 
Stochastic acyclicity, $27,28,78,79,80$, $96,99,100,106,107,114,115,116$, $124,125,134$

Stochastic ranking

moderate, 74, 79, 81, 97, 98, 107, 117 , $124,125,134$

strong, $77,79,80,82,99,107,115$, 116,117

weak, $79,80,99,105,106,107,108$, $114,116,122,124,128,133,136$. 138

Stochastic transitivity, 27, 66, 133 moderate, $27,28,29,43,45,46,81$, $82,117,124,126,133,138$ strict, 27, 28, 29, 41, 42, 45, 46, 78, $82,126,141$

strong, $27,28,29,41,42,43,45,46$, $56,57,64,65,70,77,82,99,107$. $110,112,116,117,118,121,124$, $125,126,127,133,134$

weak, $27,28,56,57,64,79,80,81$, $115,116,117,124,133$

Strong unfolding model, 8, 9, 16, 17, 18, 19, 20, 21, 28, $29,32,33,34,35,39,43,46,48$,

$49,51,68,70,127,136$

theory, $8,9,20,23,24,25,34$

Strong utility model, $17,18,58$

theory, 8, 17, 58

STST

See stochastic transitivity, strict

Symmetry condition, $35,122,124,125$, $126,127,133,135,139$

Taste testing, 1

Triangle inequality, 42, 50
Type I theory, $16,53,54,55,56,57,58$, $59,63,65$

Type II theory, 53, 54, 55, 57, 58, 59,

$61,63,64,65$

UD

See unilaterally decreasing condition บ

See unilaterally increasing condition Unfolded order, 33, 36, 37, 38, 40, 46. $50,76,77,78,96,104,105,107,118$, $119,126,127,135,136,138,140$

\section{Unfolding} deterministic, 26, 137, 139 paradigm, 1, 2, 7, 67, 139, 141 probabilistic, 8, 9, 10, 18, 22, 23, 24, $25,26,28,29,31,32,34,36,40$, $41,51,53,54,65,67,70,71,73$, $107,118,121,126,133,140,141$ representation, $2,3,8,18,19,20,24$. $26,69,139$ technique, 1, 2, 18, 19, 107, 118, 139 theory, $1,2,3,4,67,139$

Unilaterally decreasing condition, 33 , $34,35,49,76,113,122,124,125$. $127,129,132,133,137$

Unilaterally increasing condition, 33 , $35,48,51,104,105,114,125,126$, $133,135,136$

Weber law, 7 ws ranking

See stochastic ranking, weak WST

See stochastic transitivity, weak 


\title{
Patrick Bossuyt
}

\author{
Een vergelijking van \\ probabilistische ontvouwingsmodellen voor \\ data uit paarsgewijze vergelijkingen
}

Het ontvouwingsparadigma (Coombs, 1950, 1964) is in de voorbije veertig jaar een bron van inspiratie gebleken, zowel voor theorieën over keuzegedrag als voor technieken om latente structuren in data te representeren. Die populariteit neemt niet weg dat een staaltje "zuiver" ontvouwen zelden wordt gedemonstreerd; de eisen die de oorspronkelijke methode aan data stelt blijken meestal te hoog. In 1958 deed Coombs een voorstel voor een probabilistische variant van zijn ontvouwingstheorie. Na dat cerste ontwerp hebben andere auteurs in totaal nog enkele tientallen alternatieve theorieën voorgesteld.

Dit proefschrift biedt een samenvatling van deze probabilistische ontvouwingstheorieën. De samenvatting blijft beperkt tot de theorieën die werden ontworpen voor data verkregen in experimenten met paarsgewijze vergelijkingen en deze data willen afbeelden in een Euclidische ruimte. Verder bevat dit proefschrift een evaluatiestrategie die kan worden gebruikt om de validiteit van een probabilistische keuzetheorie in een keuzetaak te beoordelen. Deze strategie werd ook toegepast op een aantal datamodellen. Deze data werden verzameld in vier keuzeëxperimenten, die speciaal waren ontworpen om de toepasbaarheid van de voorgestelde probabilistische ontvouwingstheorieën te vergelijken.

In Hoofdstuk 1 worden de in de literatuur gepresenteerde voorstellen voor een probabilistische ontvouwingstheorie ondergebracht in een classificatie. Deze indeling is geïnspireerd door een bestaande en meer algemene classificatie van probabilistische keuzetheorieèn, voorgesteld door Luce en Suppes (1965). Er wordt een onderscheid gemaakt tussen random configuratie theorieën en random response theorieën. Eerstgenoemde theorieën beschouwen centrale onderdelen in de ontvouwingsrepresentatie als toevalsveranderlijken. Dat kunnen de coordinaten van de ideale alternatieven zijn, de coordinaten van de overige alternatieven, of de afstand van een alternatief tot het ideale. 
Random response theorieën zijn niet gebouwd op veronderstelde toevalsveranderlijken, maar op functionele verbanden tussen centrale elementen van een ontvouwingsrepresentatie - coordinaten of afstanden - en keuzewaarschijnlijkheden. Tot deze klasse van theorieën behoren de Fechneriaanse variant van probabilistisch ontvouwen en afleidingen van het keuzeaxioma van Luce (1959). Modellen van deze theorieën worden om hun structurele gelijkenis samen strong unfolding models genoemd. Daarnaast bevat de categorie van random response theorieën ook de midpoint unfolding theory. In deze theorie is de afstand van het ideale alternatief tot het punt van onverschilligheid tussen twee alternatieven (het middelpunt tussen beide) de belangrijkste grootheid.

In Hoofdstuk 2 wordt nagegaan wat voor eigenschappen deze theoricën van probabilistisch keuzegedrag verwachten. Tot deze eigenschappen behoren de probabilistische varianten van acycliciteit en transitiviteit in keuzen, maar ook alternatieve vertalingen van het ideale alternatief in probabilistisch keuzegedrag en enkele ordinale patronen tussen keuzewaarschijnlijkheden in een unidimensionele ruimte.

De verantwoording voor deze exploratie van eigenschappen wordt geboden in Hoofdstuk 3, waar ook het gehanteerde onderscheid tussen theorie, data en model wordt toegelicht. Hier wordt uiteengezet dat de aanwezigheid van een aantal van deze eigenschappen voor sommige theoricën een noodzakelijke of zelfs voldoende voorwaarde is voor het bestaan van een model van de theorie. Nagaan welke eigenschappen van probabilistisch keuzegedrag plausibel lijken bij een bepaald datamodel stelt een onderzocker dus in staat een duidelijker keuze te maken tussen alternatieve theorieën. In Hoofdstuk 3 wordt ook aandacht besteed aan onderzoeksdesigns bij taken met paarsgewijze keuzen.

De meeste eigenschappen van probabilistisch keuzegedrag kunnen worden omschreven als conditionele ordinale relaties voor keuzewaarschijnlijkheden. Deze relaties kunnen worden beschouwd als conditionele ordinale restricties bij het schatten van keuzewaarschijnlijkheden voor een datamodel. In de meeste gevallen komt de conditie van deze ordinale relaties neer op een rangorde van de keuzealternatieven. Als deze orde bekend is, kunnen schattingen van de waarschijnlijkheden volgens het principe van de grootste aannemelijkheid worden berekend door een isotone regressie van de keuzeproporties, met het aantal aanbiedingen van elk paar als gewicht. In Hoofdstuk 4, waar deze relaties worden beschreven, wordt een efficiënt algoritme beschreven om de isotone regressie te berckenen. 
Als de rangorde niet bekend is, kan worden gezocht naar de meest aannemelijke ordening van de alternatieven. Daar is een zoekactie voor nodig binnen de verzameling van alle mogelijke rangordeningen. Voor een vlot verloop van een dergelijke zoekactie wordt in Hoofdstuk 4 een algoritme aangeboden dat op een zuinige manier een permutatieboom doorzoekt. Tot slot wordt in Hoofdstuk 4 ook aandacht besteed aan het probleem van de statistische toets. Hoewel de generalized likelihood ratio test statistic een voor de hand liggende toetsingsgrootheid lijkt, is het voor de meeste condities niet mogelijk te bepalen wat de verdeling van deze grootheid is als aan de conditie voldaan is. Daarom wordt een Monte Carlo strategie voorgesteld: relevante kwantielen van de bestudeerde verdeling worden nonparametrisch bepaald. De hele toetsingsstrategie wordt toegepast op enkele in de literatuur gepresenteerde datamodellen.

In Hoofdstuk 5 wordt verslag uitgebracht over enkele experimenten die speciaal werden opgezet om de validiteit van de alternatieve probabilistische ontvouwingstheorieën te beoordelen, met de in de vorige hoofdstukken voorgestelde strategie. De conclusies die uit deze experimenten kunnen worden getrokken wijzen in éénzelrde richting. Sterk stochastische transitiviteit bleck weinig aannemelijk, wat een groot aantal theorieën ongeschikt maakt. De modellen van de Coombs-Zinnes-Griggs benadering blijven als de meest plausibele over. Dit zijn de modellen die het dichtst aansluiten bij Coombs' (1958) oorspronkelijke voorstel voor een theorie over probabilistisch ontvouwen. 


\section{Patrick Bossuyt}

Patrick Maria Medard Bossuyt werd geboren op 10 mei 1959 te Mortsel (België). $\mathrm{Na}$ basis- en secundair onderwijs aan het OLV College te Antwerpen (richting Latijn-Wetenschappen) studeerde hij van 1977 tot 1982 psychologie aan de Rijksuniversiteit te Gent, met als hoofdrichting theoretische en experimentele psychologie. In oktober 1982 rondde hij deze studie af met het licenciaatsdiploma in de psychologische en pedagogische wetenschappen.

In november 1983 werd hij, met steun van de Nederlandse Organisatie voor Welenschappelijk Onderzoek (NWO v/h ZWO, project 40-30), aangesteld bij de vakgroep mathematische psychologie van de Katholieke Universiteit Nijmegen. Onder begeleiding van prof.dr. E. Roskam werkte er hij aan cen vergelijking van probabilistische ontvouwingsmodellen voor data uit paarsgewijze vergelijkingen.

Sinds augustus 1987 is dhr. Bossuyt staflid in tijdelijk dienstverband van het Centrum voor Klinische Besliskunde aan de faculteit voor geneeskunde en gezondheidswetenschappen van de Erasmusuniversiteit te Rotterdam. 


\section{Stellingen}

bij het proefschrift

A comparison of

probabilistic unfolding theories

for paired comparisons data

Patrick Bossuyt

1. Of aan door keuzetheorieën aanwezig veronderstelde eigenschappen van probabilistisch keuzegedrag voldaan is, kan worden geformuleerd als een statistische hypothese; deze hypothese kan worden getoetst met behulp van de veralgemeende aannemelijkheidsverhouding.

2. Het valt te betwijfelen of aan probabilistische ontvouwingstheorieën die sterke stochastische transitiviteit, de unilateraal dalende conditie, of perfecte discriminatie van het ideaal veronderstellen enige validiteit mag worden toegekend.

3. Het unfolding probleem kan worden geformuleerd als een dubbel seriatieprobleem: als de gelijktijdige ordening van ideaalpunten en alternatieven op een onderliggende psychologische dimensie.

4. Het door Gebhardt voorgestelde algoritme voor het vinden van de isotone regressie op een partiële orde is suboptimaal.

Gebhardh F. (1970). An algorithm for monotone regression with one or more dependent variables. Biometrika, 57, 263-271.

5. Wat niet bestaat kan niet zinvol worden gemeten; het bepalen van utiliteiten (getalsmatig uitdrukkingen van relatieve verschillen in waardering) in de klinische besliskunde is in eerste instantie geen meetprobleem. 
6. Een proselitische nadruk op het normatieve status van de theorie van de maximalisatie van het subjectief verwachte nut is niet bevorderlijk gebleken voor de introductie van een oordeelkundig prescriptief gebruik van er van afgeleide besliskundige technieken.

7. Waarschijnlijk ontbreekt het ons aan cognitieve schemata om op een adequate manier met probabilistische informatie om te gaan.

8. De enige consequente interpretatie voor waarschijnlijkheden is een epistemische: als een aan voorwaarden gebonden getalsmatige uitdrukking van de mate waarin de spreker verwacht dat cen uitspraak waar zal blijken te zijn.

9. Het motct Jesu, meine Freude van J.S.Bach wordt bij voorkeur solistisch - met één stem per partij - uitgevocrd.

10.Al is het leven bezuiden de Nederlands-Belgische grens niet beter, het is er zonder twijfel aangenamer verpakt. 

This series reports new developments in all areas of psychological research-quickly, informally, and professionally. The types of research considered for publication include preliminary drafts of original papers and monographs; technical reports of high quality and broad interest; award winning theses; reports of conferences of exceptional interest, focused on a single topic. The timeliness of the manuscript is more important than its form. The publication of Recent Research in Psychology is intended to serve the international psychological community. Springer-Verlag can offer a wide distribution of documents and reports which would otherwise have a restricted audience. Once published and copyrighted, these reports can be documented in the scientific literature.

\section{Manuscript}

Manuscripts should not be less than 100 and preferably not more than 500 pages in length. Since they are reproduced by a photographic process, they must be typed with extreme care. Manuscripts submitted should be clean originals with few corrections or errors. Special symbols not on the typewriter should be inserted by hand in indelible black ink. The typescript is reduced slightly in size during reproduction with best results being obtained if each page is kept within the limit of $18 \times 26,5 \mathrm{~cm}$ ( $7 \times 10 \frac{1}{2}$ inches). On request, the publisher will supply special paper with the typing area outlined. All authors are free to use the material in other publications.

Manuscripts should be sent to Psychology Editorial, Springer-Verlag New York Inc., 175 Fifth Avenue, New York, New York 10010 or Psychology Editorial, Springer-Verlag, Tiergartenstrasse 17, D-6900 Heidelberg 1, Federal Republic of Germany. 